Título artículo / Títol article:

Study of the Stereoselectivity of the Nucleophilic Epoxidation of 3-Hydroxy-2- methylene Esters

Autores / Autors

Latorre, Antonio ; Sáez Cases, José Antonio ; Rodríguez Pastor, Santiago ; González Adelantado, Florenci Vicent

Revista:

Tetrahedron Volume 70, Issue 1, 7 January 2014

Versión / Versió:

Postprint de l'autor

Cita bibliográfica / Cita bibliogràfica (ISO 690):
LATORRE, Antonio, et al. Study of the stereoselectivity of the nucleophilic epoxidation of 3-hydroxy-2-methylene esters. Tetrahedron, 2014, 70.1: 97-102.

url Repositori UJI: http://hdl.handle.net/10234/88869 


\section{Accepted Manuscript}

Study of the Stereoselectivity of the Nucleophilic Epoxidation of 3-Hydroxy-2methylene Esters

Antonio Latorre, José A. Sáez, Santiago Rodríguez, Florenci V. González

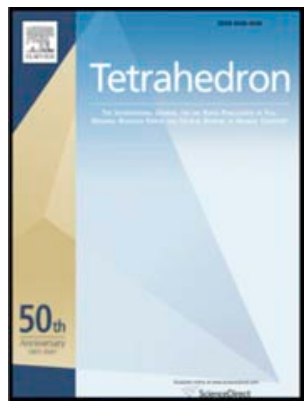

PII: $\quad$ S0040-4020(13)01702-X

DOI: $\quad$ 10.1016/j.tet.2013.11.014

Reference: TET 24991

To appear in: Tetrahedron

Received Date: 29 August 2013

Revised Date: 28 October 2013

Accepted Date: 7 November 2013

Please cite this article as: Latorre A, Sáez JA, Rodríguez S, González FV, Study of the Stereoselectivity of the Nucleophilic Epoxidation of 3-Hydroxy-2-methylene Esters, Tetrahedron (2013), doi: 10.1016/ j.tet.2013.11.014.

This is a PDF file of an unedited manuscript that has been accepted for publication. As a service to our customers we are providing this early version of the manuscript. The manuscript will undergo copyediting, typesetting, and review of the resulting proof before it is published in its final form. Please note that during the production process errors may be discovered which could affect the content, and all legal disclaimers that apply to the journal pertain. 


\title{
Study of the Stereoselectivity of the Nucleophilic Epoxidation of 3-Hydroxy-2-methylene Esters
}

\section{Antonio Latorre, Jos` A. Słez, Santiago Rodr’guez, and Florenci V. Gonzłlez*

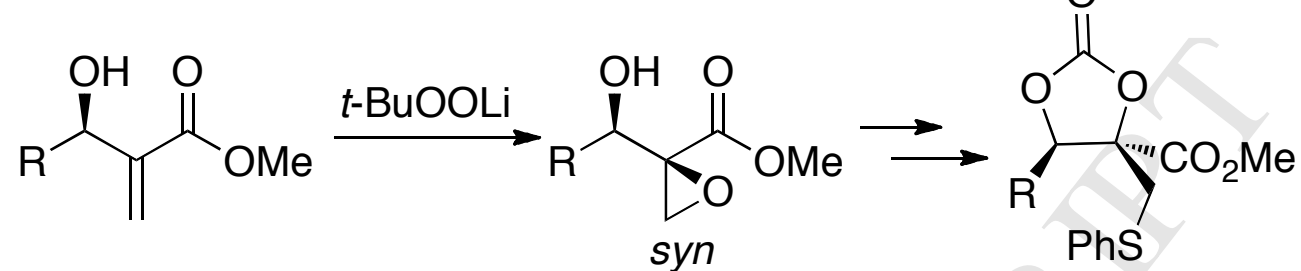 \\ Study of the Stereoselectivity of the Nucleophilic Epoxidation of 3- Hydroxy-2-methylene Esters}

\author{
Antonio Latorre, José A. Sáez, Santiago Rodríguez, Florenci V. González \\ Departament de Química Inorgànica i Orgànica, Universitat Jaume I, 12080 Castelló, Spain
}

\begin{abstract}
The diastereoselectivity of the nucleophilic epoxidation of 3-hydroxy-2-methylene esters has been studied. The 3hydroxy-2-methylene esters were obtained through a Morita-Baylis-Hillman reaction. The resulting epoxyesters were treated with thiophenol for transformation into 2,3-dihydroxy-2-((phenylthio)methyl) which upon treatment with triphosgene afforded the corresponding cyclic carbonates.
\end{abstract}

\section{Introduction}

Stereoselective synthesis of $\alpha, \beta$-epoxyesters is of considerable synthetic interest because a number of compounds can be obtained by the opening of the oxirane ring. ${ }^{1-9}$ A convenient method for the preparation of $\alpha, \beta-$ epoxyesters is via nucleophilic epoxidation of chiral $\alpha, \beta$-unsaturated esters. ${ }^{2}$ We previously reported that the nucleophilic epoxidation of $\gamma$-hydroxy- $\alpha, \beta$-unsaturated esters ${ }^{8}$ (Scheme 1 ) is a diastereoselective reaction that favor

\footnotetext{
*Corresponding author: Tel. 34 964729156. Fax: 34 964728214.E-mail: fgonzale@uji.es
} 
the syn isomer. We have also reported that the stereoselectivity depends highly on the substitution of the double bond and that high syn stereoselectivity ( $\mathrm{dr}>19: 1)$ is observed for the $\alpha$-methyl-substituted enoates ${ }^{9}$ (Scheme 1 ). Free hydroxyl group resulted to be key for the control of the stereoselectivity. The nucleophilic epoxidation of methyl 2methylene-3-tert-butyldimethylsilyloxycarboxylate esters has been recently reported by A. Myers to get the anti diastereomer with high selectivity $^{12}$ (Scheme 1). The epoxidation of Morita-Baylis-Hillman adducts is an interesting transformation because the resulting epoxides can be used in the total synthesis of interesting natural products. ${ }^{10-12}$ We now report a study of the stereoselectivity of the nucleophilic epoxidation of $\beta$-hydroxy- $\alpha$-methylene esters.

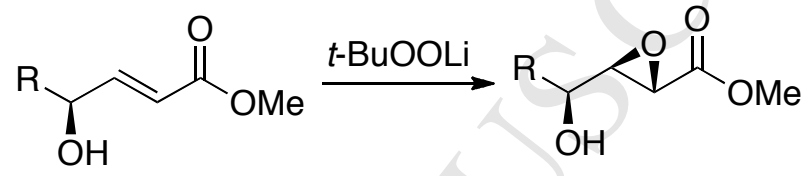
$\gamma$-hydroxy- $\alpha, \beta$-unsaturated esters

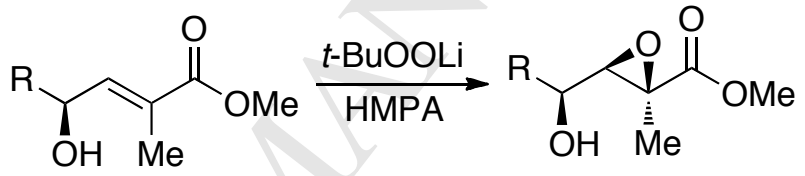
$\alpha$-methyl- $\gamma$-hydroxy- $\alpha, \beta$-unsaturated esters

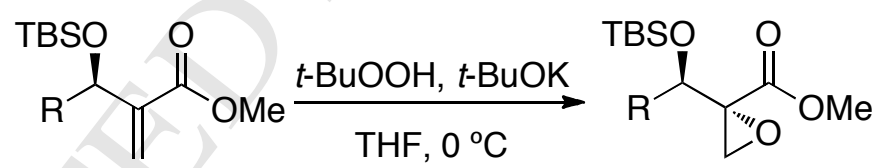
$\alpha$-methylene- $\beta$-silyloxy carboxylate esters

Scheme 1. Stereoselective nucleophilic epoxidations of unsaturated esters.

\section{Results and discussion}

We wanted to study the selectivity of epoxidation of 3-hydroxy-methylene carboxylate esters with a range of $\mathrm{R}$ alkyl and aryl groups (Scheme 2). For the preparation of the substrates, a comparison of different experimental procedures was performed as shown in Table 1. Most of the substrates were prepared in good yield using DABCO as a base and a (1:1) mixture of dioxane:water as reported. ${ }^{13}$ We obtained higher yields when the reaction was performed at higher concentrations $(10 \mathrm{M})$ than reported (see experimental section). Compounds $\mathbf{1 i}$ and $\mathbf{1 j}$ were 
obtained in best yields under solvent-free conditions and longer period of time, and compounds $1 \mathbf{m}$ and $\mathbf{1 n}$ were prepared using dimethylsulfoxide as a solvent.

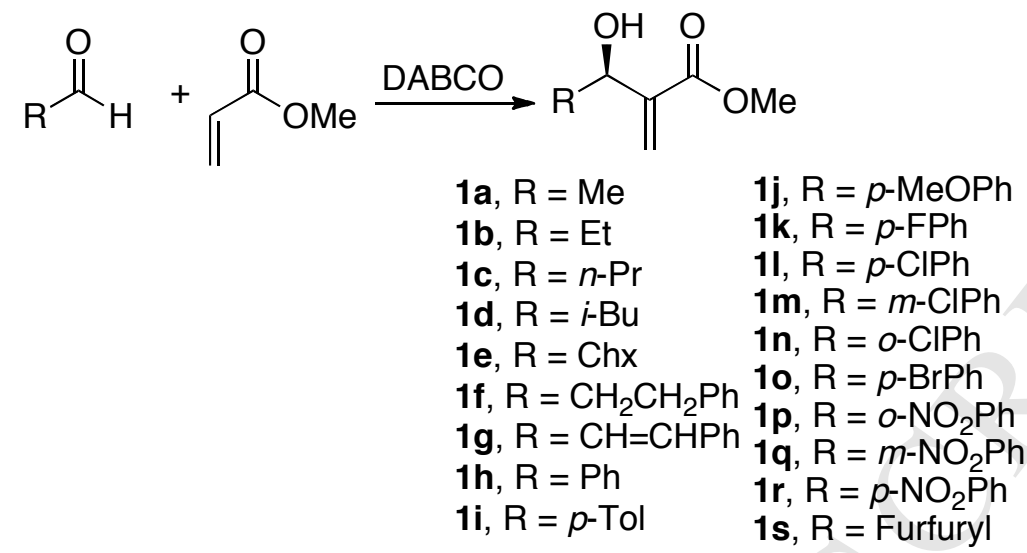

Scheme 2. Preparation of substrates.

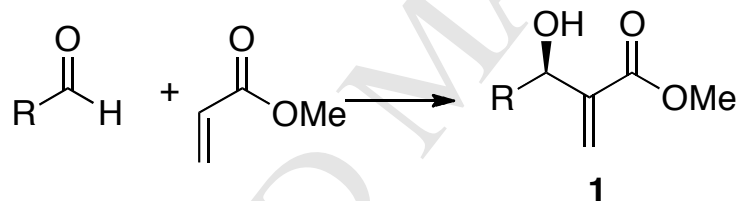

TABLE 1. Preparation of esters 1.

\begin{tabular}{|c|c|c|c|}
\hline Entry & Substrate & Conditions & Yield \\
\hline 1 & $1 \mathrm{a}$ & $\begin{array}{c}\mathrm{DABCO}, \text { dioxane: } \mathrm{H}_{2} \mathrm{O}(1: 1), \\
10 \mathrm{M}, 48 \mathrm{~h}, \mathrm{rt}\end{array}$ & 99 \\
\hline 2 & $1 b$ & $\begin{array}{c}\mathrm{DABCO}, \text { dioxane: } \mathrm{H}_{2} \mathrm{O}(1: 1) \\
10 \mathrm{M}, 48 \mathrm{~h}, \mathrm{rt}\end{array}$ & 70 \\
\hline 3 & $1 \mathrm{c}$ & $\begin{array}{c}\text { DABCO, dioxane: } \mathrm{H}_{2} \mathrm{O}(1: 1) \\
10 \mathrm{M}, 48 \mathrm{~h}, \mathrm{rt}\end{array}$ & 99 \\
\hline 4 & $1 d$ & $\begin{array}{c}\mathrm{DABCO}, \text { dioxane: } \mathrm{H}_{2} \mathrm{O}(1: 1), \\
10 \mathrm{M}, 48 \mathrm{~h}, \mathrm{rt}\end{array}$ & 85 \\
\hline 5 & $\mathbf{1 e}$ & $\begin{array}{c}\text { DABCO, dioxane: } \mathrm{H}_{2} \mathrm{O}(1: 1), \\
10 \mathrm{M}, 48 \mathrm{~h}, \mathrm{rt}\end{array}$ & 81 \\
\hline 6 & 1f & $\begin{array}{c}\text { DABCO, dioxane: } \mathrm{H}_{2} \mathrm{O}(1: 1), \\
10 \mathrm{M}, 48 \mathrm{~h}, \mathrm{rt}\end{array}$ & 99 \\
\hline 7 & $1 \mathrm{~g}$ & $\begin{array}{c}\mathrm{DABCO}, \text { dioxane: } \mathrm{H}_{2} \mathrm{O}(1: 1), \\
10 \mathrm{M}, 48 \mathrm{~h}, \mathrm{rt}\end{array}$ & 99 \\
\hline 8 & $1 \mathrm{~h}$ & $\begin{array}{c}\text { DABCO, dioxane: } \mathrm{H}_{2} \mathrm{O}(1: 1), \\
12 \mathrm{M}, 48 \mathrm{~h}, \mathrm{rt}\end{array}$ & 99 \\
\hline
\end{tabular}




\begin{tabular}{|c|c|c|c|}
\hline 9 & $\mathbf{1 i}$ & $\begin{array}{l}\text { DABCO, solvent-free } \\
4 \text { days, rt }\end{array}$ & 82 \\
\hline 10 & $\mathbf{1 j}$ & $\begin{array}{l}\text { DABCO, solvent-free } \\
5 \text { weeks, } \mathrm{rt}\end{array}$ & 77 \\
\hline 11 & $1 \mathrm{k}$ & $\begin{array}{c}\text { DABCO, dioxane: } \mathrm{H}_{2} \mathrm{O}(1: 1), \\
10 \mathrm{M}, 36 \mathrm{~h}, \mathrm{rt}\end{array}$ & 99 \\
\hline 12 & 11 & $\begin{array}{l}\text { DABCO, solvent-free } \\
5 \text { days, } \mathrm{rt}\end{array}$ & 94 \\
\hline 13 & $\mathbf{1 m}$ & $\begin{array}{l}\text { DABCO, DMSO 7M, } \\
4 \text { days, rt }\end{array}$ & 99 \\
\hline 14 & 1n & $\begin{array}{l}\text { DABCO, DMSO } 7 \mathrm{M} \\
4 \text { days, } \mathrm{rt}\end{array}$ & 99 \\
\hline 15 & 10 & $\begin{array}{c}\text { DABCO, dioxane: } \mathrm{H}_{2} \mathrm{O}(1: 1), \\
10 \mathrm{M}, 36 \mathrm{~h}, \mathrm{rt}\end{array}$ & \\
\hline 16 & $1 p$ & $\begin{array}{c}\text { DABCO, dioxane: } \mathrm{H}_{2} \mathrm{O}(1: 1), \\
10 \mathrm{M}, 16 \mathrm{~h}, \mathrm{rt}\end{array}$ & 95 \\
\hline 17 & $1 q$ & $\begin{array}{c}\text { DABCO, dioxane: } \mathrm{H}_{2} \mathrm{O}(1: 1), \\
10 \mathrm{M}, 16 \mathrm{~h}, \mathrm{rt}\end{array}$ & 87 \\
\hline 18 & $\mathbf{1 r}$ & $\begin{array}{c}\text { DABCO, dioxane: } \mathrm{H}_{2} \mathrm{O}(1: 1), \\
10 \mathrm{M}, 3 \mathrm{~h}, \mathrm{rt}\end{array}$ & 83 \\
\hline 19 & $1 \mathrm{~s}$ & $\begin{array}{c}\mathrm{DABCO}, \text { dioxane: } \mathrm{H}_{2} \mathrm{O} \\
10 \mathrm{M}, 20 \mathrm{~h}, \mathrm{rt}\end{array}$ & 85 \\
\hline
\end{tabular}

Esters 1 were epoxidized using lithium tert-butylperoxide (2 equivalents) as the oxidizing reagent in THF as solvent at $-20^{\circ} \mathrm{C} .{ }^{2,89}$ Table 2 shows that the $\mathbf{2}$ syn isomer was the major product in all cases. For the aliphatic series (compounds 1a-f), the higher steric volume of the R pendant alkyl group the higher stereoselectivity is observed (entries 1-6). When the $\mathrm{R}$ is an alkenyl group then the epoxidation reaction is not stereoselective (entry 7). Compounds $\mathbf{1 h}$-s having an aromatic group gave the corresponding syn isomer $\mathbf{2}$ in very good selectivity.

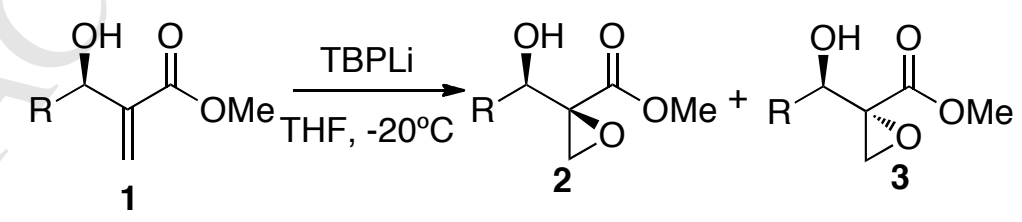

TABLE 2. Epoxidation of compounds 1.

\begin{tabular}{llll}
\hline Entry & Substrate & $\mathbf{2 / 3}$ & Yield $^{\mathrm{a}}$ \\
\hline
\end{tabular}




\begin{tabular}{|c|c|c|c|}
\hline 1 & $\mathrm{Me}$ & $67 / 33$ & 72 \\
\hline 2 & Et & $76 / 24$ & 70 \\
\hline 3 & $\mathrm{n}-\mathrm{Pr}$ & $81 / 19$ & 79 \\
\hline 4 & $\mathrm{i}-\mathrm{Bu}$ & $81 / 19$ & 71 \\
\hline 5 & Chx & $92 / 8$ & 85 \\
\hline 6 & $\mathrm{PhCH}_{2} \mathrm{CH}_{2}$ & $77 / 23$ & 59 \\
\hline 7 & $\mathrm{PhCH}=\mathrm{CH}$ & $53 / 47$ & 47 \\
\hline 8 & $\mathrm{Ph}$ & $93 / 7$ & 68 \\
\hline 9 & p-Tol & $89 / 11$ & 82 \\
\hline 10 & $\mathrm{p}-\mathrm{MeOPh}$ & $92 / 8$ & 73 \\
\hline 11 & $\mathrm{p}-\mathrm{FPh}$ & $90 / 10$ & 65 \\
\hline 12 & $\mathrm{p}-\mathrm{ClPh}$ & $84 / 16$ & 52 \\
\hline 13 & $\mathrm{~m}-\mathrm{ClPh}$ & $92 / 8$ & 38 \\
\hline 14 & o-ClPh & $92 / 8$ & 52 \\
\hline 15 & $\mathrm{BrPh}$ & $90 / 10$ & 68 \\
\hline 16 & $\mathrm{o}-\mathrm{NO} 2 \mathrm{Ph}$ & $83 / 17$ & 43 \\
\hline & $\mathrm{m}-\mathrm{NO} 2 \mathrm{Ph}$ & $80 / 20$ & 60 \\
\hline 18 & $\mathrm{p}-\mathrm{NO} 2 \mathrm{Ph}$ & $91 / 9$ & 65 \\
\hline 19 & Furfuryl & $93 / 7$ & 69 \\
\hline
\end{tabular}

We also epoxidized compound $\mathbf{1 h}$ by using oxidants other than lithium tert-butylperoxide (Table 3 ). If the reaction was carried out using tert-butyl hydrogenperoxide in the presence of substoichiometric amount of base (entry 1), then a slightly lower selectivity was observed compared to the reaction carried out using a stoichiometric amount of oxidant (entry 8, Table 2). Lithium cumylperoxide gave similar result to lithium tert-butylperoxide (entry 2). On the other hand, in the alkaline peroxides series, potassium gave poorer stereoselectivity than either lithium or sodium (entries 1 and 6-8). The yield of the epoxidation using $m$-CPBA (entry 3) was low at room temperature but it 
increased at higher temperature (entry 4), affording the syn isomer as the major one. When $m$-CPBA was used in the presence of potassium carbonate ${ }^{14}$ (entry 5), only starting material was recovered.

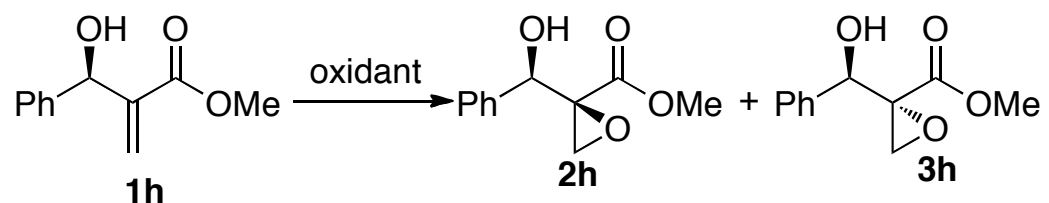

TABLE 3. Epoxidation of compound $\mathbf{1 h}$.

\begin{tabular}{cccc}
\hline Entry & Conditions $^{\mathrm{a}}$ & $\mathbf{2 h} / \mathbf{3 h}$ & ${\text { Yield }(\%)^{\mathrm{b}}}^{\mathrm{a}}$ \\
\hline 1 & TBPLi & $88 / 12$ & 66 \\
2 & CMPLi & $91 / 9$ & 72 \\
3 & mCPBA & $90 / 10$ & 28 \\
4 & mCPBA & $88 / 12$ & 80 \\
5 & mCPBA & - & NR \\
6 & TBPNa & $85 / 15$ & 41 \\
7 & TBPNa & $87 / 13$ & 62 \\
8 & TBPK & $83 / 17$ & 61 \\
\hline
\end{tabular}

${ }^{\mathrm{a}}$ For entry $1: 1.5$ equiv of TBHP, 0.8 equiv of MeLi, THF, $-20^{\circ} \mathrm{C}, 20 \mathrm{~h}$. For entry $2: 1.5$ equiv of CMHP, 1.1 equiv of MeLi, THF, $-20{ }^{\circ} \mathrm{C}, 20 \mathrm{~h} . \mathrm{For}$ entry $3: 2.1$ equiv of mCPBA, DCM, rt, $96 \mathrm{~h}$. For entry $4: 2.1$ equiv of mCPBA, $70{ }^{\circ} \mathrm{C}$ (sealed tube), $96 \mathrm{~h}$. For entry 5: 2.5 equiv of mCPBA, 1.3 equiv of $\mathrm{K}_{2} \mathrm{CO}_{3}$, DCM, rt, $96 \mathrm{~h}$. For entry $6: 2.0$ equiv of TBHP, 1.0 equiv of $t$-BuONa, THF, $0{ }^{\circ} \mathrm{C}, 3 \mathrm{~h}$. For entry $7: 2.0$ equiv of TBHP, 0.25 equiv of $t$-BuONa, THF, $0{ }^{\circ} \mathrm{C}, 3 \mathrm{~h}$. For entry $8: 2.0$ equiv of TBHP, 0.25 equiv of $t$ $\mathrm{BuOK}, \mathrm{THF}, 0{ }^{\circ} \mathrm{C}, 3 \mathrm{~h},{ }^{\mathrm{b}}$ Isolated yield of products corresponds to mixtures of syn and anti diastereomers.

The stereochemistry of epoxides $\mathbf{2 b}$ and $\mathbf{2 h}$ was confirmed by comparison with already reported data. ${ }^{12,15}$ The epoxyesters $\mathbf{2 d}, \mathbf{3 d}, \mathbf{2 e}$ and $\mathbf{3 e}$ were transformed into cyclic carbonates through a one-pot sequence: treatment with thiophenol in the presence of a base which resulted in the opening of the oxirane ring and then addition of triphosgene to give carbonates $\mathbf{4 , 5 , 6}$ and 7, respectively (Scheme 3). The stereochemical assignment of the carbonates was performed by NOE experiments (Scheme 3). Carbonates 4 and 6 gave NOE between H-5 and methyl ester whilst 5 and 7 gave NOE between H-5 and methylene from the (phenylthio)methyl group. 

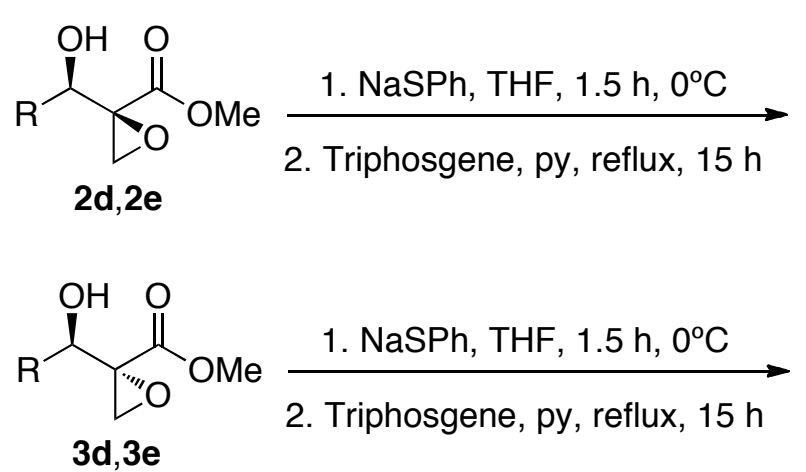<smiles>[R]C1OC(=O)O[C@@]1(CS)C(=O)OC</smiles>

PhS 4,6

$3 d, 3 e$
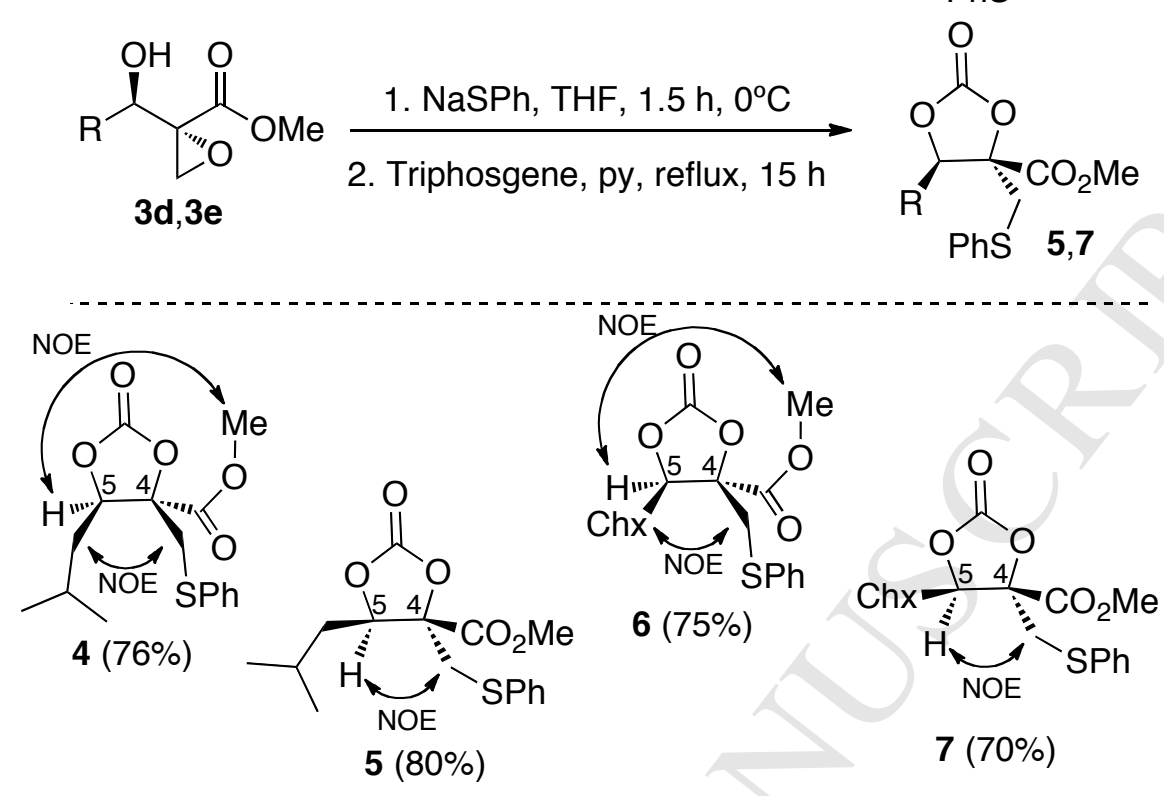

Scheme 3. Cyclic carbonates 4-7.

\section{Conclusions}

In summary, the diastereoselectivity of the nucleophilic epoxidation of 3-hydroxy-2-methylene esters has been studied. The syn isomer was the major one in all cases. The resulting 3-hydroxy 2-epoxyesters were treated with thiophenol for transformation into 2,3-dihydroxy-2-((phenylthio)methyl) which upon treatment with triphosgene afforded the corresponding cyclic carbonates.

\section{Experimental Section}

General Experimental Methods. All solvents used in reactions were freshly distilled from appropriate drying agents before use. ${ }^{1} \mathrm{H}$ NMR spectra and ${ }^{13} \mathrm{C}$ NMR spectra were measured in $\mathrm{CDCl}_{3}\left({ }^{1} \mathrm{H}, 7.24 \mathrm{ppm} ;{ }^{13} \mathrm{C} 77.0\right.$ ppm) solution at $30{ }^{\circ} \mathrm{C}$ on a $300 \mathrm{MHz}$ or a $500 \mathrm{MHz} \mathrm{NMR}$ spectrometer. IR spectra were recorded as oil films or $\mathrm{KBr}$ discs or $\mathrm{NaCl}$ pellets on a FT-IR spectrometer. EM Science Silica Gel 60 was used for column chromatography while TLC was 
performed with precoated plates (Kieselgel $60, \mathrm{~F}_{254}, 0.25 \mathrm{~mm}$ ). Unless otherwise specified, all reactions were carried out under argon atmosphere with magnetic stirring.

\section{General experimental procedure for the preparation of compounds 1a-s:}

To a solution of aldehyde $(1 \mathrm{mmol})$ in dioxane-water $(1: 1)(0.1 \mathrm{~mL})$ was added methyl acrylate $(3 \mathrm{mmol})$ and DABCO $(1 \mathrm{mmol})$. The reaction was monitored by TLC. Upon completion, water $(70 \mathrm{~mL})$ was added and poured onto a separatory funnel and extracted with ethyl ether or dichloromethane ( $3 \times 30 \mathrm{~mL})$, dried over anhydrous $\mathrm{Na}_{2} \mathrm{SO}_{4}$, filtered, and concentrated under reduced pressure. The crude was purified through chromatography (silicagel, hexanes/ethyl acetate $(8: 2),(6: 4))$ to afford the desired compound.

Methyl 3-hydroxy-2-methylenebutanoate 1a. ${ }^{16}{ }^{1} \mathrm{H}$ NMR $\left(500 \mathrm{MHz}, \mathrm{CDCl}_{3}\right) \delta 6.19(1 \mathrm{H}, \mathrm{s}), 5.81(1 \mathrm{H}, \mathrm{s}), 4.59(1 \mathrm{H}$, $\mathrm{q}, \mathrm{J}=6.5 \mathrm{~Hz}), 3.76(3 \mathrm{H}, \mathrm{s}), 2.61(1 \mathrm{H}, \mathrm{br} \mathrm{s}), 1.36(3 \mathrm{H}, \mathrm{d}, \mathrm{J}=6.5 \mathrm{~Hz}) .{ }^{13} \mathrm{C}$ NMR $\left(125 \mathrm{MHz}, \mathrm{CDCl}_{3}\right) \delta 167.1,143.6$, $124.0,67.2,51.8,22.1 \mathrm{ppm}$.

Methyl 3-hydroxy-2-methylenepentanoate $1 \mathrm{~b} .{ }^{17}{ }^{1} \mathrm{H}$ NMR $\left(500 \mathrm{MHz}, \mathrm{CDCl}_{3}\right) \delta 6.22(1 \mathrm{H}, \mathrm{s}), 5.78(\mathrm{~s}, 1 \mathrm{H}), 4.31$ $(1 \mathrm{H}, \mathrm{t}, \mathrm{J}=7.0 \mathrm{~Hz}), 3.76(3 \mathrm{H}, \mathrm{s}), 2.43(1 \mathrm{H}, \mathrm{br} \mathrm{s}), 1.73-1.61(2 \mathrm{H}, \mathrm{m}), 0.93(3 \mathrm{H}, \mathrm{t}, \mathrm{J}=7.4 \mathrm{~Hz}) .{ }^{13} \mathrm{C} \mathrm{NMR}(125 \mathrm{MHz}$, $\left.\mathrm{CDCl}_{3}\right) \delta 167.0,142.3,124.7,72.0,51.5,29.0,10.0 \mathrm{ppm}$.

Methyl 3-hydroxy-2-methylenehexanoate 1c. ${ }^{18}{ }^{1} \mathrm{H}$ NMR $\left(500 \mathrm{MHz}, \mathrm{CDCl}_{3}\right) \delta 6.20(1 \mathrm{H}, \mathrm{s}), 5.78(1 \mathrm{H}, \mathrm{s}), 4.38(1 \mathrm{H}$, $\mathrm{t}, \mathrm{J}=6.5 \mathrm{~Hz}), 3.76(3 \mathrm{H}, \mathrm{s}), 2.41(1 \mathrm{H}, \mathrm{br} \mathrm{s}), 1.63-1.58(2 \mathrm{H}, \mathrm{m}), 1.49-1.45(1 \mathrm{H}, \mathrm{m}), 1.31-1.38(1 \mathrm{H}, \mathrm{m}), 0.90(3 \mathrm{H}, \mathrm{t}, \mathrm{J}=$ $6.7 \mathrm{~Hz}) .{ }^{13} \mathrm{C}$ NMR $\left(125 \mathrm{MHz}, \mathrm{CDCl}_{3}\right) \delta 167.0,142.5,124.7,71.3,52.0,38.5,19.0,14.0 \mathrm{ppm}$.

Methyl 3-hydroxy-5-methyl-2-methylenehexanoate 1d. ${ }^{18}{ }^{1} \mathrm{H}$ NMR $\left(500 \mathrm{MHz}, \mathrm{CDCl}_{3}\right) \delta 6.18(1 \mathrm{H}, \mathrm{s}), 5.78(1 \mathrm{H}, \mathrm{s})$, $4.45(1 \mathrm{H}, \mathrm{dd}, \mathrm{J}=8.5,4.3 \mathrm{~Hz}), 3.76(3 \mathrm{H}, \mathrm{s}), 2.40(1 \mathrm{H}, \mathrm{br} \mathrm{s}), 1.80-1.75(1 \mathrm{H}, \mathrm{m}), 1.58-1.51(1 \mathrm{H}, \mathrm{m}), 1.44-1.38(1 \mathrm{H}, \mathrm{m})$, $0.92(6 \mathrm{H}, \mathrm{m}) .{ }^{13} \mathrm{C}$ NMR $\left(125 \mathrm{MHz}, \mathrm{CDCl}_{3}\right) \delta$ 167.0, 142.8, 124.6, 71.3, 69.9, 51.9, 45.5, 24.8, 23.3, $21.8 \mathrm{ppm}$.

Methyl 2-(cyclohexyl(hydroxy)methyl)acrylate 1 e. ${ }^{19}{ }^{1} \mathrm{H}$ NMR $\left(500 \mathrm{MHz}, \mathrm{CDCl}_{3}\right) \delta 6.23(1 \mathrm{H}, \mathrm{s}), 5.71(1 \mathrm{H}, \mathrm{s}), 4.06$ $(1 \mathrm{H}, \mathrm{d}, \mathrm{J}=7.2 \mathrm{~Hz}), 3.76(3 \mathrm{H}, \mathrm{s}), 2.44(1 \mathrm{H}, \mathrm{br} \mathrm{s}), 1.94(1 \mathrm{H}, \mathrm{m}), 1.50-1.76(5 \mathrm{H}, \mathrm{m}), 1.24-0.92(5 \mathrm{H}, \mathrm{m}) .{ }^{13} \mathrm{C}$ NMR $(125$ $\left.\mathrm{MHz}, \mathrm{CDCl}_{3}\right) \delta 167.0,141.2,126.0,53.4,52.0,42.4,29.8,28.1,26.3,26.1,25.9 \mathrm{ppm}$. 
Methyl 3-hydroxy-2-methylene-5-phenylpentanoate 1f. ${ }^{20}{ }^{1} \mathrm{H}$ NMR $\left(500 \mathrm{MHz}, \mathrm{CDCl}_{3}\right) \delta$ 7.30-7.17 (5H, m), 6.24 $(1 \mathrm{H}, \mathrm{s}), 5.81(1 \mathrm{H}, \mathrm{s}), 4.42(1 \mathrm{H}, \mathrm{dd}, \mathrm{J}=7.5,5.7 \mathrm{~Hz}), 3.77(3 \mathrm{H}, \mathrm{s}), 2.85-2.79(1 \mathrm{H}, \mathrm{m}), 2.73-2.69(1 \mathrm{H}, \mathrm{m}), 2.42(1 \mathrm{H}, \mathrm{br}$ s), 2.00-1.95 (1H, m). ${ }^{13} \mathrm{C} \mathrm{NMR}\left(125 \mathrm{MHz}, \mathrm{CDCl}_{3}\right) \delta$ 167.0, 141.8, 128.5, 125.9, 125.0, 70.1, 51.8, 38.0, $32.0 \mathrm{ppm}$. (E)-methyl 3-hydroxy-2-methylene-5-phenylpent-4-enoate 1g. ${ }^{19}{ }^{1} \mathrm{H} \mathrm{NMR}\left(500 \mathrm{MHz}, \mathrm{CDCl}_{3}\right) \delta$ 7.39-7.22 (5H, m), $6.67(1 \mathrm{H}, \mathrm{d}, \mathrm{J}=16.0 \mathrm{~Hz}), 6.29(2 \mathrm{H}, \mathrm{m}), 5.91(1 \mathrm{H}, \mathrm{s}), 5.13(1 \mathrm{H}, \mathrm{m}), 3.78(3 \mathrm{H}, \mathrm{s}), 2.97(1 \mathrm{H}, \mathrm{br} \mathrm{s}) .{ }^{13} \mathrm{C} \mathrm{NMR}(125 \mathrm{MHz}$, $\left.\mathrm{CDCl}_{3}\right) \delta 166.7,141.3,136.5,131.5,129.2,128.5,127.8,126.6,125.8,72.1,52.0 \mathrm{ppm}$.

Methyl 2-(hydroxy(phenyl)methyl)acrylate $1 \mathbf{h} .{ }^{18}{ }^{1} \mathrm{H} \mathrm{NMR}\left(500 \mathrm{MHz}, \mathrm{CDCl}_{3}\right) \delta$ 7.38-7.26 (5H, m), 6.33 (1H, s), $5.83(1 \mathrm{H}, \mathrm{s}), 5.56(1 \mathrm{H}, \mathrm{s}), 3.72(3 \mathrm{H}, \mathrm{s}) .{ }^{13} \mathrm{C} \mathrm{NMR}\left(125 \mathrm{MHz}, \mathrm{CDCl}_{3}\right) \delta 166.7,142.3,141.6,128.3,127.7,126.8$, $125.6,72.7,51.8 \mathrm{ppm}$.

Methyl 2-(hydroxy(p-tolyl)methyl)acrylate 1i. ${ }^{18}{ }^{1} \mathrm{H} \mathrm{NMR}\left(300 \mathrm{MHz}, \mathrm{CDCl}_{3}\right) \delta 7.26(2 \mathrm{H}, \mathrm{d}, \mathrm{J}=8.0 \mathrm{~Hz}), 7.15(2 \mathrm{H}$, $\mathrm{d}, \mathrm{J}=8.0 \mathrm{~Hz}), 6.32(1 \mathrm{H}, \mathrm{s}), 5.85(1 \mathrm{H}, \mathrm{s}), 5.53(1 \mathrm{H}, \mathrm{s}), 3.71(3 \mathrm{H}, \mathrm{s}), 2.34(3 \mathrm{H}, \mathrm{s}) \cdot{ }^{13} \mathrm{C} \mathrm{NMR}\left(125 \mathrm{MHz}, \mathrm{CDCl}_{3}\right) \delta$ $166.8,142.1,138.4,137.5,129.1,126.5,125.8,73.1,51.9,21.1 \mathrm{ppm}$.

Methyl 2-(hydroxy(4-methoxyphenyl)methyl)acrylate $\mathbf{1 j}^{20}{ }^{1} \mathrm{H} \mathrm{NMR}\left(300 \mathrm{MHz}, \mathrm{CDCl}_{3}\right) \delta 7.28(2 \mathrm{H}, \mathrm{d}, \mathrm{J}=8.8 \mathrm{~Hz})$, $6.86(2 \mathrm{H}, \mathrm{d}, \mathrm{J}=8.7 \mathrm{~Hz}), 6.31(1 \mathrm{H}, \mathrm{s}), 5.84(1 \mathrm{H}, \mathrm{s}), 5.52(1 \mathrm{H}, \mathrm{s}), 3.79(3 \mathrm{H}, \mathrm{s}), 3.71(3 \mathrm{H}, \mathrm{s}) .{ }^{13} \mathrm{C} \mathrm{NMR}(75 \mathrm{MHz}$, $\left.\mathrm{CDCl}_{3}\right) \delta 166.8,159.2,142.2,133.5,127.9,125.5,113.8,72.7,55.2,51.9 \mathrm{ppm}$.

Methyl 2-((4-fluorophenyl)(hydroxy)methyl)acrylate $1 \mathbf{k}^{20}{ }^{1} \mathrm{H} \mathrm{NMR}\left(500 \mathrm{MHz}, \mathrm{CDCl}_{3}\right) \delta 7.33(2 \mathrm{H}, \mathrm{dd}, \mathrm{J}=8.5$, $5.5 \mathrm{~Hz}), 7.01(2 \mathrm{H}, \mathrm{t}, \mathrm{J}=8.7 \mathrm{~Hz}), 6.32(1 \mathrm{H}, \mathrm{s}), 5.82(1 \mathrm{H}, \mathrm{s}), 5.53(1 \mathrm{H}, \mathrm{s}), 3.73(3 \mathrm{H}, \mathrm{s}), 3.02(1 \mathrm{H}, \mathrm{br} \mathrm{s}) .{ }^{13} \mathrm{C}$ NMR $(125$ $\left.\mathrm{MHz}, \mathrm{CDCl}_{3}\right) \delta 166.6,162.3(\mathrm{~d}, \mathrm{~J}=245 \mathrm{~Hz}), 141.9,137.0,128.3(\mathrm{dd}, \mathrm{J}=7.2,21.3 \mathrm{~Hz}), 126.0(\mathrm{dd}, \mathrm{J}=15.0,21.3 \mathrm{~Hz})$, $115.2(\mathrm{dd}, \mathrm{J}=12.5,22.5 \mathrm{~Hz}), 72.6,52.2 \mathrm{ppm}$.

Methyl 2-((4-chlorophenyl)(hydroxy)methyl)acrylate $11 .{ }^{18}{ }^{1} \mathrm{H}$ NMR $\left(500 \mathrm{MHz}, \mathrm{CDCl}_{3}\right) \delta 7.54(1 \mathrm{H}, \mathrm{m}), 7.34(1 \mathrm{H}$, m), 7.21-7.30 (2H, m), $6.32(1 \mathrm{H}, \mathrm{s}), 5.97(1 \mathrm{H}, \mathrm{s}), 5.58(1 \mathrm{H}, \mathrm{m}), 3.76(3 \mathrm{H}, \mathrm{s}), 3.26(1 \mathrm{H}, \mathrm{br} \mathrm{s}) .{ }^{13} \mathrm{C} \mathrm{NMR}(125 \mathrm{MHz}$, $\left.\mathrm{CDCl}_{3}\right) \delta 166.6,141.6,139.8,133.6,128.6,127.9,126.3,72.7,52.0 \mathrm{ppm}$. 
Methyl 2-((3-chlorophenyl)(hydroxy)methyl)acrylate $1 \mathbf{1 m}^{20}{ }^{1} \mathrm{H} \mathrm{NMR}\left(500 \mathrm{MHz}, \mathrm{CDCl}_{3}\right) \delta 7.37(1 \mathrm{H}, \mathrm{s}), 7.26(3 \mathrm{H}$, m), $6.34(1 \mathrm{H}, \mathrm{s}), 5.83(\mathrm{~s}, 1 \mathrm{H}), 5.51(1 \mathrm{H}, \mathrm{s}), 3.72(\mathrm{~s}, 3 \mathrm{H}), 3.03(1 \mathrm{H}, \mathrm{br} \mathrm{s}) .{ }^{13} \mathrm{C} \mathrm{NMR}\left(125 \mathrm{MHz}, \mathrm{CDCl}_{3}\right) \delta$ 166.5, 143.4, $141.4,134.4,129.7,127.9,126.7,126.6,124.7,72.7,52.0 \mathrm{ppm}$.

Methyl 2-((2-chlorophenyl)(hydroxy)methyl)acrylate $1{ }^{20}{ }^{1} \mathrm{H}$ NMR $\left(500 \mathrm{MHz}, \mathrm{CDCl}_{3}\right) \delta 7.54(1 \mathrm{H}, \mathrm{m}), 7.34(1 \mathrm{H}$, m), 7.21-7.30 (2H, m), $6.32(1 \mathrm{H}, \mathrm{s}), 5.97(1 \mathrm{H}, \mathrm{s}), 5.58(1 \mathrm{H}, \mathrm{m}), 3.76(3 \mathrm{H}, \mathrm{s}), 3.25(1 \mathrm{H}, \mathrm{br} \mathrm{s}) .{ }^{13} \mathrm{C}$ NMR $(125 \mathrm{MHz}$, $\left.\mathrm{CDCl}_{3}\right) \delta 166.9,140.9,134.5,132.8,128.9,128.1,127.0,126.8,68.9,52.0 \mathrm{ppm}$.

Methyl 2-((4-bromophenyl)(hydroxy)methyl)acrylate $10^{20}{ }^{1} \mathrm{H}$ NMR $\left(300 \mathrm{MHz}, \mathrm{CDCl}_{3}\right) \delta 7.47(2 \mathrm{H}, \mathrm{m}), 7.25(2 \mathrm{H}$, m), $6.33(1 \mathrm{H}, \mathrm{s}), 5.82(1 \mathrm{H}, \mathrm{s}), 5.51(1 \mathrm{H}, \mathrm{m}), 3.73(3 \mathrm{H}, \mathrm{s}), 3.04(1 \mathrm{H}, \mathrm{br} \mathrm{s}) .{ }^{13} \mathrm{C} \mathrm{NMR}\left(75 \mathrm{MHz}, \mathrm{CDCl}_{3}\right) \delta 166.4,141.9$, $140.6,131.4,128.6,125.9,121.6,71.9,51.9 \mathrm{ppm}$.

Methyl 2-((2-nitrophenyl)(hydroxy)methyl)acrylate 1p. ${ }^{18}{ }^{1} \mathrm{H}$ NMR $\left(500 \mathrm{MHz}, \mathrm{CDCl}_{3}\right) \delta 7.95(1 \mathrm{H}, \mathrm{dd}, \mathrm{J}=8.2$, $1.3 \mathrm{~Hz}), 7.75(1 \mathrm{H}, \mathrm{dd}, \mathrm{J}=7.9,1.3 \mathrm{~Hz}), 7.64(1 \mathrm{H}, \mathrm{td}, \mathrm{J}=7.7,1.3 \mathrm{~Hz}), 7.46(1 \mathrm{H}, \mathrm{td}, \mathrm{J}=8.5,1.4 \mathrm{~Hz}), 6.37(1 \mathrm{H}, \mathrm{s}), 6.20$ $(1 \mathrm{H}, \mathrm{s}), 5.73(1 \mathrm{H}, \mathrm{s}), 3.73(3 \mathrm{H}, \mathrm{s}), 3.35(1 \mathrm{H}, \mathrm{br} \mathrm{s}) .{ }^{13} \mathrm{C} \mathrm{NMR}\left(75 \mathrm{MHz}, \mathrm{CDCl}_{3}\right) \delta 163.8,145.6,138.5,133.7,130.8$, $126.3,126.0,123.7,121.9,64.7,49.5 \mathrm{ppm}$.

Methyl 2-((3-nitrophenyl)(hydroxy)methyl)acrylate 1q. ${ }^{18} \mathrm{H} \mathrm{NMR}\left(500 \mathrm{MHz}, \mathrm{CDCl}_{3}\right) \delta 8.26(1 \mathrm{H}, \mathrm{m}), 8.14(1 \mathrm{H}$, ddd, $\mathrm{J}=8.2,2.3,1.2 \mathrm{~Hz}), 7.75(1 \mathrm{H}, \mathrm{m}), 7.52(1 \mathrm{H}, \mathrm{t}, \mathrm{J}=7.92 \mathrm{~Hz}), 6.41(1 \mathrm{H}, \mathrm{s}), 5.89(1 \mathrm{H}, \mathrm{s}), 5.63(1 \mathrm{H}, \mathrm{s}), 3.75(3 \mathrm{H}, \mathrm{s})$, 3.25 (1H, br s). ${ }^{13} \mathrm{C}$ NMR (125 MHz, $\left.\mathrm{CDCl}_{3}\right) \delta 166.2,148.3,143.7,141.0,132.8,129.5,126.9,122.8,121.7,72.4$, $52.3 \mathrm{ppm}$.

Methyl 2-((4-nitrophenyl)(hydroxy)methyl)acrylate $1 r^{18}{ }^{1} \mathrm{H} \mathrm{NMR}\left(500 \mathrm{MHz}, \mathrm{CDCl}_{3}\right) \delta 8.18(2 \mathrm{H}, \mathrm{d}, \mathrm{J}=10.9 \mathrm{~Hz})$, $7.56(2 \mathrm{H}, \mathrm{d}, \mathrm{J}=10.9 \mathrm{~Hz}), 6.38(1 \mathrm{H}, \mathrm{s}), 5.86(1 \mathrm{H}, \mathrm{s}), 5.62(1 \mathrm{H}, \mathrm{m}), 3.73(3 \mathrm{H}, \mathrm{s}), 3.32(1 \mathrm{H}, \mathrm{br} \mathrm{s}) .{ }^{13} \mathrm{C}$ NMR $(125 \mathrm{MHz}$, $\left.\mathrm{CDCl}_{3}\right) \delta 166.4,148.6,143.7,141.0,127.3,127.2,123.6,72.7,52.2 \mathrm{ppm}$.

Methyl 2-(furan-2-yl(hydroxy)methyl)acrylate 1s. ${ }^{18}{ }^{1} \mathrm{H} \mathrm{NMR}\left(500 \mathrm{MHz}, \mathrm{CDCl}_{3}\right) \delta 7.35(1 \mathrm{H}, \mathrm{s}), 6.37(1 \mathrm{H}, \mathrm{s}), 6.31$ $(1 \mathrm{H}, \mathrm{m}), 6.24(1 \mathrm{H}, \mathrm{m}), 5.93(1 \mathrm{H}, \mathrm{s}), 5.57(1 \mathrm{H}, \mathrm{s}), 3.74(3 \mathrm{H}, \mathrm{s}), 3.21(1 \mathrm{H}, \mathrm{br} \mathrm{s}) .{ }^{13} \mathrm{C} \mathrm{NMR}\left(125 \mathrm{MHz}, \mathrm{CDCl}_{3}\right) \delta 166.4$, $154.1,142.3,143.7,139.5,126.7,110.4,107.1,67.2,52.0 \mathrm{ppm}$. 
To a $-78{ }^{\circ} \mathrm{C}$ cold THF (3.5 mL) was added TBHP (3.3 $\mathrm{M}$ in toluene) $(2 \mathrm{mmol})$ and then methyllithium (1.6M in hexanes) $(1.7 \mathrm{mmol})$. The resulting mixture was stirred at $-78{ }^{\circ} \mathrm{C}$ for $15 \mathrm{~min}$ and then a solution of compound 1 (1 $\mathrm{mmol}$ ) in THF $\left(2 \mathrm{~mL}\right.$ ) was added drop wise and then the mixture was left at $-20{ }^{\circ} \mathrm{C}$ (fridge) for $20 \mathrm{~h}$. Then solid $\mathrm{Na}_{2} \mathrm{SO}_{3}(120 \mathrm{mg})$ was added in one portion and stirred for $15 \mathrm{~min}$, then diluted with water and extracted with $\mathrm{Et}_{2} \mathrm{O}(3$ x $30 \mathrm{~mL}$ ), the organic layers were washed (brine), dried $\left(\mathrm{Na}_{2} \mathrm{SO}_{4}\right)$, and concentrated. The crude oil was purified through chromatography (silica-gel, hexanes/EtOAc (7:3) and (1:1)).

Methyl 2-(1-hydroxyethyl)oxirane-2-carboxylate 2a/3a. (yield= $167 \mathrm{mg}, 99 \%)$ (Ratio of diastereomers $67 / 33$ ). ${ }^{1} \mathrm{H}$ $\operatorname{NMR}\left(500 \mathrm{MHz}, \mathrm{CDCl}_{3}\right) \delta 4.33(1 \mathrm{H}, \mathrm{q}, \mathrm{J}=6.6 \mathrm{~Hz})$ (minor), $4.16(1 \mathrm{H}, \mathrm{q}, \mathrm{J}=6.4 \mathrm{~Hz})$ (major), $3.71(3 \mathrm{H}, \mathrm{s}), 3.10(1 \mathrm{H}$, $\mathrm{d}, \mathrm{J}=5.9 \mathrm{~Hz}$ ) (major), $4.64(1 \mathrm{H}, \mathrm{d}, \mathrm{J}=6.1 \mathrm{~Hz})($ minor), $2.99(1 \mathrm{H}, \mathrm{d}, \mathrm{J}=6.0 \mathrm{~Hz})(\operatorname{minor}), 2.96(1 \mathrm{H}, \mathrm{d}, \mathrm{J}=5.8 \mathrm{~Hz})$ (major), $2.08\left(1 \mathrm{H}\right.$, br s), $1.31(3 \mathrm{H}, \mathrm{d}, \mathrm{J}=6.4 \mathrm{~Hz})$ (major), $1.29(3 \mathrm{H}, \mathrm{d}, \mathrm{J}=6.6 \mathrm{~Hz})$ (minor). ${ }^{13} \mathrm{C} \mathrm{NMR}(125 \mathrm{MHz}$, $\left.\mathrm{CDCl}_{3}\right) \delta 170.0$ (minor), 169.9 (major), 65.1 (major), 64.9 (minor), 59.6 (minor), 59.0 (major), 52.5 (major), 52.4

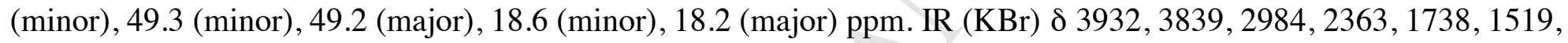
$1382,1285,1173,1095,971,913,853 \mathrm{~cm}^{-1}$. HRMS m/z calcd. for $\mathrm{C}_{6} \mathrm{H}_{10} \mathrm{O}_{4} \mathrm{Na}\left[\mathrm{M}+\mathrm{Na}^{+}\right]: 169.0477$, found: 169.0478 . Methyl 2-(1-hydroxypropyl)oxirane-2-carboxylate 2b/3b. (yield= $128 \mathrm{mg}, 70 \%$ ) (Ratio of diastereomers 76/24). ${ }^{1} \mathrm{H}$ NMR $\left(500 \mathrm{MHz}, \mathrm{CDCl}_{3}\right) \delta 3.75(1 \mathrm{H}, \mathrm{m}), 3.71(3 \mathrm{H}, \mathrm{s}), 3.12(1 \mathrm{H}, \mathrm{d}, \mathrm{J}=6.0 \mathrm{~Hz}), 2.98(1 \mathrm{H}, \mathrm{d}, \mathrm{J}=6.0 \mathrm{~Hz}), 2.55(1 \mathrm{H}$, br s), $1.72(1 \mathrm{H}, \mathrm{m}), 1.48(1 \mathrm{H}, \mathrm{m}), 0.98(3 \mathrm{H}, \mathrm{t}, \mathrm{J}=6.7 \mathrm{~Hz}) .{ }^{13} \mathrm{C} \mathrm{NMR}\left(125 \mathrm{MHz}, \mathrm{CDCl}_{3}\right) \delta 170.0,71.0,50.1,52.5,49.6$, 26.0, 9.9 ppm. IR (KBr) $\delta 3770,3457,2939,2360,1869,1637,1541,1440,1348,1197,1139,1055,950,758 \mathrm{~cm}^{-1}$. HRMS $m / z$ calcd. for $\mathrm{C}_{7} \mathrm{H}_{12} \mathrm{O}_{4} \mathrm{Na}\left[\mathrm{M}+\mathrm{Na}^{+}\right]: 183.0633$, found: 183.0636 .

(Yield 2c/3c $=99 \%)$

syn-Methyl 2-(1-hydroxybutyl)oxirane-2-carboxylate $2 c .{ }^{1} \mathrm{H}$ NMR $\left(500 \mathrm{MHz}, \mathrm{CDCl}_{3}\right) \delta 3.84-3.87(1 \mathrm{H}, \mathrm{m}), 3.78$ $(3 \mathrm{H}, \mathrm{s}), 3.12(1 \mathrm{H}, \mathrm{d}, \mathrm{J}=5.9 \mathrm{~Hz}), 2.98(1 \mathrm{H}, \mathrm{d}, \mathrm{J}=5.9 \mathrm{~Hz}), 1.69-1.40(4 \mathrm{H}, \mathrm{m}), 0.94(3 \mathrm{H}, \mathrm{t}, \mathrm{J}=7.2 \mathrm{~Hz}) .{ }^{13} \mathrm{C} \mathrm{NMR}(125$ $\left.\mathrm{MHz}_{,} \mathrm{CDCl}_{3}\right) \delta 170.0,69.3,58.3,52.4,49.6,35.0,18.7,13.8$ ppm. IR (KBr) $\delta 3649,2960,2361,1740,1560,1457$, 1382, 1197, 1139, 1077, 983, $760 \mathrm{~cm}^{-1}$. HRMS m/z calcd. for $\mathrm{C}_{8} \mathrm{H}_{14} \mathrm{O}_{4} \mathrm{Na}\left[\mathrm{M}+\mathrm{Na}^{+}\right]$: 197.0790, found: 197.0786 .

anti-Methyl 2-(1-hydroxybutyl)oxirane-2-carboxylate 3c. ${ }^{1} \mathrm{H}$ NMR $\left(500 \mathrm{MHz}, \mathrm{CDCl}_{3}\right) \delta 4.12-4.10(1 \mathrm{H}, \mathrm{m}), 3.78$ $(3 \mathrm{H}, \mathrm{s}), 3.08(1 \mathrm{H}, \mathrm{d}, \mathrm{J}=6.0 \mathrm{~Hz}), 2.98(1 \mathrm{H}, \mathrm{d}, \mathrm{J}=6.0 \mathrm{~Hz}), 1.77(1 \mathrm{H}, \mathrm{br} \mathrm{s}), 1.61-1.37(4 \mathrm{H}, \mathrm{m}), 0.94(3 \mathrm{H}, \mathrm{t}, \mathrm{J}=7.1 \mathrm{~Hz})$. 
${ }^{13} \mathrm{C}$ NMR $\left(125 \mathrm{MHz}, \mathrm{CDCl}_{3}\right) \delta 170.0,69.0,59.4,52.5,49.3,35.1,18.9,13.7$ ppm. IR (KBr) $\delta 3466,2960,1739$, $1639,1567,1441,1356,1287,1212,1197,1138,1129,1036,982,957 \mathrm{~cm}^{-1}$.

$($ Yield 2d/3d $=71 \%)$

syn-Methyl 2-(1-hydroxy-3-methylbutyl)oxirane-2-carboxylate 2d. ${ }^{1} \mathrm{H} \mathrm{NMR}\left(500 \mathrm{MHz}, \mathrm{CDCl}_{3}\right) \delta 3.92(1 \mathrm{H}, \mathrm{dd}, \mathrm{J}$ $=3.8,9.2 \mathrm{~Hz}), 3.78(3 \mathrm{H}, \mathrm{s}), 3.12(1 \mathrm{H}, \mathrm{d}, \mathrm{J}=5.9 \mathrm{~Hz}), 2.98(1 \mathrm{H}, \mathrm{d}, \mathrm{J}=5.9 \mathrm{~Hz}), 1.93-1.86(1 \mathrm{H}, \mathrm{m}), 1.41-1.51(2 \mathrm{H}, \mathrm{m})$, $0.95(6 \mathrm{H}, \mathrm{t}, \mathrm{J}=6.5 \mathrm{~Hz}) .{ }^{13} \mathrm{C} \mathrm{NMR}\left(125 \mathrm{MHz}, \mathrm{CDCl}_{3}\right) \delta 170.0,68.0,58.5,52.5,49.6,41.8,24.4,23.5,21.4 \mathrm{ppm} . \mathrm{IR}$ $(\mathrm{KBr}) \delta 3743,2956,2361,1738,1438,1368,1171,1116,1078,994,919,864,758 \mathrm{~cm}^{-1}$. HRMS $\mathrm{m} / \mathrm{z}$ calcd. for $\mathrm{C}_{9} \mathrm{H}_{16} \mathrm{O}_{4} \mathrm{Na}\left[\mathrm{M}+\mathrm{Na}^{+}\right]: 211.0946$, found: 211.0942.

anti-Methyl 2-(1-hydroxy-3-methylbutyl)oxirane-2-carboxylate 3d. ${ }^{1} \mathrm{H}$ NMR $\left(500 \mathrm{MHz}, \mathrm{CDCl}_{3}\right) \delta 4.20(1 \mathrm{H}$, dd, J $=3.8,9.2 \mathrm{~Hz}), 3.77(3 \mathrm{H}, \mathrm{s}), 3.09(1 \mathrm{H}, \mathrm{d}, \mathrm{J}=5.9 \mathrm{~Hz}), 2.98(1 \mathrm{H}, \mathrm{d}, \mathrm{J}=5.9 \mathrm{~Hz}), 2.06-1.96(1 \mathrm{H}, \mathrm{br} \mathrm{s}), 1.76-1.82(1 \mathrm{H}, \mathrm{m})$, 1.48-1.51 (1H, m), 1.27-1.35 (1H, m), $0.95(6 \mathrm{H}, \mathrm{t}, \mathrm{J}=6.5 \mathrm{~Hz}) .{ }^{13} \mathrm{C}$ NMR $\left(125 \mathrm{MHz}, \mathrm{CDCl}_{3}\right) \delta 170.0,67.5,59.6,52.5$, 49.3, 42.0, 24.4, 23.5, 21.3 ppm. IR (KBr) $\delta$ 3491, 2957, 2393, 1738, 1440, 1368, 1184, 1115, 1094, 993, 919, 879 $\mathrm{cm}^{-1}$.

syn-Methyl 2-(cyclohexyl(hydroxy)methyl)oxirane-2-carboxylate $2 \mathrm{e} .{ }^{1} \mathrm{H} \mathrm{NMR}\left(500 \mathrm{MHz}, \mathrm{CDCl}_{3}\right) \delta 3.78(3 \mathrm{H}, \mathrm{s})$, $3.39(1 \mathrm{H}, \mathrm{d}, \mathrm{J}=6.7 \mathrm{~Hz}), 3.11(1 \mathrm{H}, \mathrm{d}, \mathrm{J}=5.9 \mathrm{~Hz}), 2.96(1 \mathrm{H}, \mathrm{d}, \mathrm{J}=5.9 \mathrm{~Hz}), 2.12(1 \mathrm{H}, \mathrm{br} \mathrm{s}), 1.88(1 \mathrm{H}, \mathrm{m}), 1.75-1.63(5 \mathrm{H}$, m), 1.26-1.03 (5H, m). ${ }^{13} \mathrm{C}$ NMR $\left(125 \mathrm{MHz}, \mathrm{CDCl}_{3}\right) \delta 170.1,75.1,57.3,52.4,49.7,41.2,29.3,28.2,26.2,26.0,25.8$ ppm. IR (KBr) $\delta 3799,2930,2669,2342,1741,1377,1306,1200,1124,1087,1030,932,761 \mathrm{~cm}^{-1}$. HRMS m/z calcd. for $\mathrm{C}_{11} \mathrm{H}_{18} \mathrm{O}_{4} \mathrm{Na}\left[\mathrm{M}+\mathrm{Na}^{+}\right]$: 237.1103, found: 237.1105 .

anti-Methyl 2-(cyclohexyl(hydroxy)methyl)oxirane-2-carboxylate $3 \mathrm{e} .{ }^{1} \mathrm{H} \mathrm{NMR}\left(500 \mathrm{MHz}, \mathrm{CDCl}_{3}\right) \delta 3.77(3 \mathrm{H}, \mathrm{s})$, $3.69(1 \mathrm{H}, \mathrm{d}, \mathrm{J}=6.5 \mathrm{~Hz}), 3.02(1 \mathrm{H}, \mathrm{d}, \mathrm{J}=5.9 \mathrm{~Hz}), 2.97(1 \mathrm{H}, \mathrm{d}, \mathrm{J}=5.9 \mathrm{~Hz}), 1.94(1 \mathrm{H}, \mathrm{m}), 1.78-1.64(5 \mathrm{H}, \mathrm{m}), 1.30-0.94$ $(5 \mathrm{H}, \mathrm{m}) .{ }^{13} \mathrm{C} \mathrm{NMR}\left(125 \mathrm{MHz}, \mathrm{CDCl}_{3}\right) \delta 169.8,74.5,65.7,58.7,52.5,49.6,41.5,29.6,28.2,26.1,25.8,15.1 \mathrm{ppm} . \mathrm{IR}$ (KBr) $\delta 3752,2936,2668,2341,1740,1422,1232,1153,1104,1069,1052,974,957 \mathrm{~cm}^{-1}$.

syn-Methyl 2-(1-hydroxy-3-phenylpropyl)oxirane-2-carboxylate $2 \mathrm{f} .{ }^{1} \mathrm{H}$ NMR $\left(500 \mathrm{MHz}, \mathrm{CDCl}_{3}\right) \delta$ 7.33-7.20 (5H, $\mathrm{m}), 3.95(1 \mathrm{H}, \mathrm{m}), 3.77(3 \mathrm{H}, \mathrm{s}), 3.12(1 \mathrm{H}, \mathrm{d}, \mathrm{J}=6.0 \mathrm{~Hz}), 2.95(1 \mathrm{H}, \mathrm{d}, \mathrm{J}=6.0 \mathrm{~Hz}), 2.93-2.88(1 \mathrm{H}, \mathrm{m}), 2.77-2.65(1 \mathrm{H}$, 
m), 2.20-2.01 (1H, m), 1.82-1.93 (1H, m). ${ }^{13} \mathrm{C} \mathrm{NMR}\left(125 \mathrm{MHz}, \mathrm{CDCl}_{3}\right) \delta 170.0,141.5,128.4,126.1,68.9,58.3$, 52.6, 49.3, 34.8, 31.7 ppm. IR (KBr) $\delta 3873,3063,3003,2924,2364,1748,1290,1240,1132,1075,754,701 \mathrm{~cm}^{-1}$.

anti-Methyl 2-(1-hydroxy-3-phenylpropyl)oxirane-2-carboxylate 3f. ${ }^{1} \mathrm{H}$ NMR $\left(500 \mathrm{MHz}, \mathrm{CDCl}_{3}\right) \delta$ 7.30-7.17 $(5 \mathrm{H}, \mathrm{m}), 4.11(1 \mathrm{H}, \mathrm{d}, \mathrm{J}=9.3 \mathrm{~Hz}), 3.76(3 \mathrm{H}, \mathrm{s}), 3.05(1 \mathrm{H}, \mathrm{d}, \mathrm{J}=6.0 \mathrm{~Hz}), 3.00(1 \mathrm{H}, \mathrm{d}, \mathrm{J}=6.0 \mathrm{~Hz}), 2.93-2.87(1 \mathrm{H}, \mathrm{m})$, 2.75-2.69 $(1 \mathrm{H}, \mathrm{m}), 1.99-1.93(1 \mathrm{H}, \mathrm{m}), 1.89-1.82(1 \mathrm{H}, \mathrm{m}), 1.54(1 \mathrm{H}, \mathrm{br} \mathrm{s}) .{ }^{13} \mathrm{C} \mathrm{NMR}\left(125 \mathrm{MHz}, \mathrm{CDCl}_{3}\right) \delta 170.0$, $141.3,128.4,126.0,68.9,59.1,52.3,49.3,34.5,31.8 \mathrm{ppm}$.

(E)-Methyl 2-(1-hydroxy-3-phenylallyl)oxirane-2-carboxylate $\quad \mathbf{2 g} / \mathbf{3 g} . \quad($ yield $=103 \mathrm{mg}, \quad 47 \%$ ) (Ratio of diastereomers 53/47). ${ }^{1} \mathrm{H}$ NMR $\left(500 \mathrm{MHz}, \mathrm{CDCl}_{3}\right) \delta 7.40-7.24(\mathrm{~m}, 5 \mathrm{H}), 6.74(1 \mathrm{H}, \mathrm{d}, \mathrm{J}=16.0 \mathrm{~Hz})$ (major and minor), $6.27(1 \mathrm{H}, \mathrm{dd}, \mathrm{J}=6.3,12.0 \mathrm{~Hz})$ (major), $6.23(1 \mathrm{H}, \mathrm{dd}, \mathrm{J}=5.8,13.2 \mathrm{~Hz})(\operatorname{minor}), 4.85(1 \mathrm{H}, \mathrm{dd}, \mathrm{J}=6.3,1.3 \mathrm{~Hz})(\mathrm{minor})$, $4.71(1 \mathrm{H}, \mathrm{dd}, \mathrm{J}=6.5,1.2 \mathrm{~Hz})$ (major), $3.80(3 \mathrm{H}, \mathrm{s})$ (major), $3.79(3 \mathrm{H}, \mathrm{s})$ (minor), $3.15(1 \mathrm{H}, \mathrm{d}, \mathrm{J}=5.9 \mathrm{~Hz})$ (major), 3.13 $(1 \mathrm{H}, \mathrm{d}, \mathrm{J}=6.1 \mathrm{~Hz})$ (minor), $3.06(1 \mathrm{H}, \mathrm{d}, \mathrm{J}=6.1 \mathrm{~Hz})$ (minor), $3.00(1 \mathrm{H}, \mathrm{d}, \mathrm{J}=5.9 \mathrm{~Hz})$ (major). ${ }^{13} \mathrm{C} \mathrm{NMR}(125 \mathrm{MHz}$, $\mathrm{CDCl}_{3}$ ) $\delta 169.8$ (minor), 169.7 (major), 136.2 (minor), 136.1 (major), 133.5 (major), 133.1 (minor), 128.6, 128.1, 128.0, 126.7 (major and minos), 125.9 (minor), 125.6 (major), 70.7 (major), 70.0 (minor), 59.0 (minor), 58.5 (major), 52.7, 52.6 (major and minor), 49.9 (major), 49.2 (minor) ppm. HRMS $\mathrm{m} / z$ calcd. for $\mathrm{C}_{13} \mathrm{H}_{14} \mathrm{O}_{4} \mathrm{Na}\left[\mathrm{M}+\mathrm{Na}^{+}\right]$: 257.0790, found: 257.0792 .

syn-Methyl 2-(hydroxy(phenyl)methyl)oxirane-2-carboxylate $2 \mathbf{h} .{ }^{10}{ }^{1} \mathrm{H} \mathrm{NMR}\left(500 \mathrm{MHz}, \mathrm{CDCl}_{3}\right) \delta$ 7.43-7.30 (5H, m), $5.18(1 \mathrm{H}, \mathrm{s}), 3.73(3 \mathrm{H}, \mathrm{s}), 3.12(1 \mathrm{H}, \mathrm{d}, \mathrm{J}=5.9 \mathrm{~Hz}), 2.86(1 \mathrm{H}, \mathrm{d}, \mathrm{J}=5.9 \mathrm{~Hz}) .{ }^{13} \mathrm{C} \mathrm{NMR}\left(125 \mathrm{MHz}, \mathrm{CDCl}_{3}\right) \delta 169.7$,

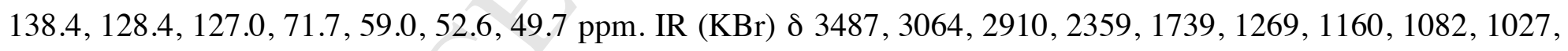
947, $757 \mathrm{~cm}^{-1}$. HRMS $\mathrm{m} / z$ calcd. for $\mathrm{C}_{11} \mathrm{H}_{12} \mathrm{O}_{4} \mathrm{Na}\left[\mathrm{M}+\mathrm{Na}^{+}\right]: 231.0633$, found: 231.0632 .

syn-Methyl 2-(hydroxy(p-tolyl)methyl)oxirane-2-carboxylate 2i. ${ }^{1} \mathrm{H} N \mathrm{NR}\left(500 \mathrm{MHz}, \mathrm{CDCl}_{3}\right) \delta 7.29(2 \mathrm{H}, \mathrm{d}, \mathrm{J}=$ $8.0 \mathrm{~Hz}), 7.26(2 \mathrm{H}, \mathrm{d}, \mathrm{J}=8.0 \mathrm{~Hz}), 5.15(1 \mathrm{H}, \mathrm{s}), 3.72(3 \mathrm{H}, \mathrm{s}), 3.11(1 \mathrm{H}, \mathrm{d}, \mathrm{J}=5.9 \mathrm{~Hz}), 2.86(1 \mathrm{H}, \mathrm{d}, \mathrm{J}=5.9 \mathrm{~Hz}) .{ }^{13} \mathrm{C} \mathrm{NMR}$ $\left(125 \mathrm{MHz}, \mathrm{CDCl}_{3}\right) \delta 169.8,137.9,135.5,128.9,127.0,71.4,59.1,52.6,49.6,21.1 \mathrm{ppm} . \mathrm{IR}(\mathrm{KBr}) \delta 3502,3005$, 2923, 1743, 1197, 1125, 1020, 943, 837, 765, $686 \mathrm{~cm}^{-1}$. HRMS $\mathrm{m} / z$ calcd. for $\mathrm{C}_{12} \mathrm{H}_{14} \mathrm{O}_{4} \mathrm{Na}\left[\mathrm{M}+\mathrm{Na}^{+}\right]: 245.0790$, found: 245.0787 . 
syn-Methyl 2-(hydroxy(4-methoxyphenyl)methyl)oxirane-2-carboxylate $\mathbf{2 j} \cdot{ }^{1} \mathrm{H} \mathrm{NMR}\left(500 \mathrm{MHz}, \mathrm{CDCl}_{3}\right) \delta 7.33$ $(2 \mathrm{H}, \mathrm{d}, \mathrm{J}=8.8 \mathrm{~Hz}), 6.87(2 \mathrm{H}, \mathrm{d}, \mathrm{J}=8.8 \mathrm{~Hz}), 5.16(1 \mathrm{H}, \mathrm{s}), 3.79(3 \mathrm{H}, \mathrm{s}), 3.67(3 \mathrm{H}, \mathrm{s}), 3.12(1 \mathrm{H}, \mathrm{d}, \mathrm{J}=6.0 \mathrm{~Hz}), 2.85(1 \mathrm{H}$, $\mathrm{d}, \mathrm{J}=6.0 \mathrm{~Hz}) .{ }^{13} \mathrm{C} \mathrm{NMR}\left(125 \mathrm{MHz}, \mathrm{CDCl}_{3}\right) \delta 169.8,159.5,130.4,128.5,113.8,71.2,59.1,55.2,52.6,49.5 \mathrm{ppm} . \mathrm{IR}$ $(\mathrm{KBr}) \delta 3493,3003,2910,1742,1197,1124,1031,978,917,836,756 \mathrm{~cm}^{-1}$. HRMS $\mathrm{m} / z$ calcd. for $\mathrm{C}_{12} \mathrm{H}_{14} \mathrm{O}_{5} \mathrm{Na}$ $\left[\mathrm{M}+\mathrm{Na}^{+}\right]: 261.0739$, found: 261.0738 .

syn-Methyl 2-((4-fluorophenyl)(hydroxy)methyl)oxirane-2-carboxylate 2k. ${ }^{1} \mathrm{H} \mathrm{NMR}\left(500 \mathrm{MHz}, \mathrm{CDCl}_{3}\right) \delta 7.40$ $(2 \mathrm{H}, \mathrm{dd}, \mathrm{J}=8.5,5.5 \mathrm{~Hz}), 7.03(2 \mathrm{H}, \mathrm{t}, \mathrm{J}=8.7 \mathrm{~Hz}), 5.15(1 \mathrm{H}, \mathrm{s}), 3.73(3 \mathrm{H}, \mathrm{s}), 3.13(1 \mathrm{H}, \mathrm{d}, \mathrm{J}=6.0 \mathrm{~Hz}), 2.85(1 \mathrm{H}, \mathrm{d}, \mathrm{J}=$ $6.0 \mathrm{~Hz}) .{ }^{13} \mathrm{C}$ NMR $\left(125 \mathrm{MHz}, \mathrm{CDCl}_{3}\right) \delta 169.7,162.2(\mathrm{~d}, \mathrm{~J}=245 \mathrm{~Hz}), 134.2,129.0(\mathrm{dd}, \mathrm{J}=7.2,21.3 \mathrm{~Hz}), 115.2(\mathrm{dd}, \mathrm{J}=$ 12.5, 22.5Hz), 71.1, 65.8, 52.7, 49.6 ppm. IR (KBr) $\delta$ 3477, 3070, 2958, 2342, 1737, 1509, 1398, 1271, 1197, 1128 , $1045,980,842,756 \mathrm{~cm}^{-1}$. HRMS $\mathrm{m} / z$ calcd. for $\mathrm{C}_{11} \mathrm{H}_{11} \mathrm{FO}_{4} \mathrm{Na}\left[\mathrm{M}+\mathrm{Na}^{+}\right]: 249.0539$, found: 249.0535 .

syn-Methyl 2-((4-chlorophenyl)(hydroxy)methyl)oxirane-2-carboxylate $21 .{ }^{1} \mathrm{H}$ NMR $\left(500 \mathrm{MHz}, \mathrm{CDCl}_{3}\right) \delta 7.37$ $(2 \mathrm{H}, \mathrm{d}, \mathrm{J}=8.0 \mathrm{~Hz}), 7.32(2 \mathrm{H}, \mathrm{d}, \mathrm{J}=8.0 \mathrm{~Hz}), 5.29(1 \mathrm{H}, \mathrm{s}), 3.73(3 \mathrm{H}, \mathrm{s}), 3.14(1 \mathrm{H}, \mathrm{d}, \mathrm{J}=6.0 \mathrm{~Hz}), 2.88(1 \mathrm{H}, \mathrm{d}, \mathrm{J}=$ $6.0 \mathrm{~Hz}) .{ }^{13} \mathrm{C}$ NMR $\left(125 \mathrm{MHz}, \mathrm{CDCl}_{3}\right) \delta 169.6,137.0,134.1,128.6,128.5,71.2,58.7,52.7,49.7 \mathrm{ppm} . \mathrm{IR}(\mathrm{KBr}) \delta$ $3518,3001,2929,1723,1411,1287,1160,1107,1049,982,920,756 \mathrm{~cm}^{-1}$. HRMS m/z calcd. for $\mathrm{C}_{11} \mathrm{H}_{11} \mathrm{ClO}_{4} \mathrm{Na}$ $\left[\mathrm{M}+\mathrm{Na}^{+}\right]: 265.0244$, found: 265.0245 .

syn-Methyl 2-((3-chlorophenyl)(hydroxy)methyl)oxirane-2-carboxylate $2 \mathbf{m} .{ }^{1} \mathrm{H} N M R\left(500 \mathrm{MHz}, \mathrm{CDCl}_{3}\right) \delta 7.44$ $(1 \mathrm{H}, \mathrm{s}), 7.32(3 \mathrm{H}, \mathrm{m}), 5.10(1 \mathrm{H}, \mathrm{s}), 3.73(3 \mathrm{H}, \mathrm{s}), 3.16(1 \mathrm{H}, \mathrm{d}, \mathrm{J}=6.0 \mathrm{~Hz}), 2.90(1 \mathrm{H}, \mathrm{d}, \mathrm{J}=6.0 \mathrm{~Hz}) .{ }^{13} \mathrm{C}$ NMR $(125$ $\left.\mathrm{MHz}, \mathrm{CDCl}_{3}\right) \delta 169.6,140.6,134.3,129.6,128.4,127.1,125.3,71.3,58.7,52.8,49.8$ ppm. IR (KBr) $\delta 3466,3020$, $2964,1736,1463,1264,1154,1170,1083,962,918,877 \mathrm{~cm}^{-1}$.

syn-Methyl 2-((2-chlorophenyl)(hydroxy)methyl)oxirane-2-carboxylate 2 n. ${ }^{1} \mathrm{H}$ NMR $\left(500 \mathrm{MHz}, \mathrm{CDCl}_{3}\right) \delta 7.54$ $(1 \mathrm{H}, \mathrm{m}), 7.33(1 \mathrm{H}, \mathrm{m}), 7.24-7.29(2 \mathrm{H}, \mathrm{m}), 6.04(1 \mathrm{H}, \mathrm{s}), 3.82(3 \mathrm{H}, \mathrm{s}), 3.06(1 \mathrm{H}, \mathrm{d}, \mathrm{J}=6.0 \mathrm{~Hz}), 2.35(1 \mathrm{H}, \mathrm{d}, \mathrm{J}=6.0 \mathrm{~Hz})$. ${ }^{13} \mathrm{C}$ NMR $\left(125 \mathrm{MHz}, \mathrm{CDCl}_{3}\right) \delta 169.9,135.0,132.9,129.5,127.9,126.9,67.9,58.6,52.9,50.5 \mathrm{ppm} . \mathrm{IR}(\mathrm{KBr}) \delta$ $3741,3019,2938,1734,1472,1390,1297,1195,1064,1028,1028,758,741 \mathrm{~cm}^{-1}$.

syn-Methyl 2-((4-bromophenyl)(hydroxy)methyl)oxirane-2-carboxylate $20 .{ }^{1} \mathrm{H}$ NMR $\left(500 \mathrm{MHz}, \mathrm{CDCl}_{3}\right) \delta 7.48$ $(2 \mathrm{H}, \mathrm{d}, \mathrm{J}=8.0 \mathrm{~Hz}), 7.32(2 \mathrm{H}, \mathrm{d}, \mathrm{J}=8.0 \mathrm{~Hz}), 5.29(1 \mathrm{H}, \mathrm{s}), 3.73(3 \mathrm{H}, \mathrm{s}), 3.14(1 \mathrm{H}, \mathrm{d}, \mathrm{J}=6.0 \mathrm{~Hz}), 2.88(1 \mathrm{H}, \mathrm{d}, \mathrm{J}=$ 
$6.0 \mathrm{~Hz}) .{ }^{13} \mathrm{C}$ NMR $\left(125 \mathrm{MHz}, \mathrm{CDCl}_{3}\right) \delta 169.6,137.5,131.5,129.0,122.2,71.2,58.7,52.7,48.9 \mathrm{ppm} . \mathrm{IR}(\mathrm{KBr}) \delta$ $3711,3077,2957,2360,1923,1592,1728,1460,1333,1286,1196,1127,1049,935,755 \mathrm{~cm}^{-1}$. HRMS m/z calcd. for $\mathrm{C}_{11} \mathrm{H}_{11} \mathrm{BrO}_{4} \mathrm{Na}\left[\mathrm{M}+\mathrm{Na}^{+}\right]$: 308.9738 , found: 308.9735 .

syn-Methyl 2-((2-nitrophenyl)(hydroxy)methyl)oxirane-2-carboxylate 2p. ${ }^{1} \mathrm{H}$ NMR $\left(500 \mathrm{MHz}, \mathrm{CDCl}_{3}\right) \delta 7.96$ $(1 \mathrm{H}, \mathrm{dd}, \mathrm{J}=8.2,1.3 \mathrm{~Hz}), 7.79(1 \mathrm{H}, \mathrm{dd}, \mathrm{J}=7.9,1.3 \mathrm{~Hz}), 7.65(1 \mathrm{H}, \mathrm{td}, \mathrm{J}=7.7,1.3 \mathrm{~Hz}), 7.48(1 \mathrm{H}, \mathrm{td}, \mathrm{J}=8.5,1.4 \mathrm{~Hz})$, $6.17(1 \mathrm{H}, \mathrm{s}), 3.82(3 \mathrm{H}, \mathrm{s}), 3.13(1 \mathrm{H}, \mathrm{d}, \mathrm{J}=6.0 \mathrm{~Hz}), 2.37(1 \mathrm{H}, \mathrm{d}, \mathrm{J}=6.0 \mathrm{~Hz}) .{ }^{13} \mathrm{C}$ NMR $\left(125 \mathrm{MHz}, \mathrm{CDCl}_{3}\right) \delta 169.6$, 148.3, 133.3, 129.8, 129.1, 124.6, 66.8, 58.1, 53.1, 51.0 ppm. IR (KBr) $\delta 3648,3093,2957,1725,1440,1357,1267$, $1200,1156,1053,947,747 \mathrm{~cm}^{-1}$.

syn-Methyl 2-((3-nitrophenyl)(hydroxy)methyl)oxirane-2-carboxylate 2q. ${ }^{1} \mathrm{H}$ NMR $\left(500 \mathrm{MHz}, \mathrm{CDCl}_{3}\right) \delta 8.33$ $(1 \mathrm{H}, \mathrm{m}), 8.17(1 \mathrm{H}, \mathrm{ddd}, \mathrm{J}=8.2,2.3,1.2 \mathrm{~Hz}), 7.81(1 \mathrm{H}, \mathrm{m}), 7.52(1 \mathrm{H}, \mathrm{t}, \mathrm{J}=7.92 \mathrm{~Hz}), 5.19(1 \mathrm{H}, \mathrm{s}), 3.74(3 \mathrm{H}, \mathrm{s}), 3.22$ $(1 \mathrm{H}, \mathrm{d}, \mathrm{J}=6.0 \mathrm{~Hz}), 2.95(1 \mathrm{H}, \mathrm{d}, \mathrm{J}=6.0 \mathrm{~Hz}) .{ }^{13} \mathrm{C} \mathrm{NMR}\left(125 \mathrm{MHz}, \mathrm{CDCl}_{3}\right) \delta 169.4,148.2,133.3,129.2,123.2,122.1$, 71.0, 65.7, 52.8, 49.7 ppm. IR (KBr) ठ 3712, 3092, 3006, 2957, 2876, 1735, 1441, 1353, 1289, 1163, 1096, 976, 935, $866,758 \mathrm{~cm}^{-1}$.

syn-Methyl 2-((4-nitrophenyl)(hydroxy)methyl)oxirane-2-carboxylate 2 r. ${ }^{1} \mathrm{H}$ NMR $\left(500 \mathrm{MHz}, \mathrm{CDCl}_{3}\right) \delta 8.22$ $(2 \mathrm{H}, \mathrm{d}, \mathrm{J}=8.0 \mathrm{~Hz}), 7.65(2 \mathrm{H}, \mathrm{d}, \mathrm{J}=8.0 \mathrm{~Hz}), 5.13(1 \mathrm{H}, \mathrm{s}), 3.73(3 \mathrm{H}, \mathrm{s}), 3.48(1 \mathrm{H}, \mathrm{d}, \mathrm{J}=6.0 \mathrm{~Hz}), 2.94(1 \mathrm{H}, \mathrm{d}, \mathrm{J}=$ $6.0 \mathrm{~Hz}) .{ }^{13} \mathrm{C}$ NMR $\left(125 \mathrm{MHz}, \mathrm{CDCl}_{3}\right) \delta 169.5,146.0,128.1,127.8,123.4,71.4,58.4,53.0,49.9 \mathrm{ppm} . \mathrm{IR}(\mathrm{KBr}) \delta$ $3902,3087,2958,2342,1925,1715,1517,1442,1221,1096,1053,946,777 \mathrm{~cm}^{-1}$. HRMS $\mathrm{m} / z$ calcd. for $\mathrm{C}_{11} \mathrm{H}_{11} \mathrm{NO}_{6} \mathrm{Na}\left[\mathrm{M}+\mathrm{Na}^{+}\right]: 276.0484$, found: 276.0482 .

syn-Methyl 2-(furan-2-yl(hydroxy)methyl)oxirane-2-carboxylate 2 s. ${ }^{1} \mathrm{H}$ NMR $\left(500 \mathrm{MHz}, \mathrm{CDCl}_{3}\right) \delta 7.38(1 \mathrm{H}, \mathrm{s})$, $7.26(1 \mathrm{H}, \mathrm{s}), 6.39(1 \mathrm{H}, \mathrm{m}), 6.33(1 \mathrm{H}, \mathrm{m}), 5.29(1 \mathrm{H}, \mathrm{s}), 3.75(3 \mathrm{H}, \mathrm{s}), 3.23(1 \mathrm{H}, \mathrm{d}, \mathrm{J}=6.0 \mathrm{~Hz}), 3.05(1 \mathrm{H}, \mathrm{d}, \mathrm{J}=6.0 \mathrm{~Hz})$. ${ }^{13} \mathrm{C}$ NMR $\left(125 \mathrm{MHz}, \mathrm{CDCl}_{3}\right) \delta 169.3,125.6,142.5,110.4,107.7,64.5,52.6,49.0 \mathrm{ppm} . \mathrm{IR}(\mathrm{KBr}) \delta 3932,3153$, $3004,2957,1734,1633,1359,1231,1048,975,753 \mathrm{~cm}^{-1}$.

\section{General experimental procedure for the preparation of cyclic carbonates:}

An ice-bath cold suspension of sodium hydride (60\% in mineral oil) $(1.12 \mathrm{mmol})$ in THF $(1 \mathrm{~mL})$ was treated with 
thiophenol $(2.25 \mathrm{mmol})$. The mixture was stirred at room temperature for $15 \mathrm{~min}$ and then a solution of the epoxyester $2(0.75 \mathrm{mmol})$ in THF $(1 \mathrm{~mL})$ was added drop wise and the mixture was stirred at room temperature for $1.5 \mathrm{~h}$. Then was treated with pyridine $(0.22 \mathrm{mmol})$ and triphosgene $(0.48 \mathrm{mmol})$. The mixture was refluxed for $15 \mathrm{~h}$. Then brine was added and extracted with $\mathrm{Et}_{2} \mathrm{O}(3 \times 20 \mathrm{~mL})$, the organic layers were washed (brine), dried $\left(\mathrm{Na}_{2} \mathrm{SO}_{4}\right)$, and concentrated. The crude oil was purified through chromatography (silica-gel, hexanes/EtOAc (8:2) and (7:3)).

syn-Methyl 5-isobutyl-2-oxo-4-((phenylthio)methyl)-1,3-dioxolane-4-carboxylate $4 .{ }^{1} \mathrm{H} \mathrm{NMR}\left(500 \mathrm{MHz}, \mathrm{CDCl}_{3}\right)$

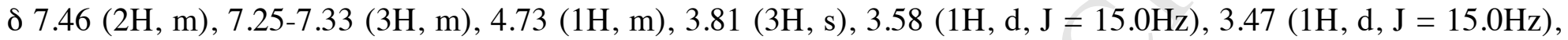
$1.77(1 \mathrm{H}, \mathrm{m}), 1.44(1 \mathrm{H}, \mathrm{m}), 1.35(1 \mathrm{H}, \mathrm{m}), 0.92(3 \mathrm{H}, \mathrm{d}, \mathrm{J}=6.5 \mathrm{~Hz}), 0.84(3 \mathrm{H}, \mathrm{d}, \mathrm{J}=6.5 \mathrm{~Hz}) .{ }^{13} \mathrm{C}$ NMR $(125 \mathrm{MHz}$ $\left.\mathrm{CDCl}_{3}\right) \delta 167.7,152.7,134.7,131.3,129.3,127.7,86.6,80.4,53.2,39.6,38.6,25.1,23.0,21.2 \mathrm{ppm} . \mathrm{IR}(\mathrm{KBr}) \delta$ $3059,2959,1811,1743,1626,1540,1470,1387,1306,1200,1116,1025,968,746 \mathrm{~cm}^{-1}$. HRMS $\mathrm{m} / \mathrm{z}$ calcd. for $\mathrm{C}_{16} \mathrm{H}_{20} \mathrm{O}_{5} \mathrm{SNa}\left[\mathrm{M}+\mathrm{Na}^{+}\right]: 347.0929$, found: 347.0929 .

anti-Methyl 5-isobutyl-2-oxo-4-((phenylthio)methyl)-1,3-dioxolane-4-carboxylate $5 .{ }^{1} \mathrm{H}$ NMR $\left(500 \mathrm{MHz}, \mathrm{CDCl}_{3}\right)$

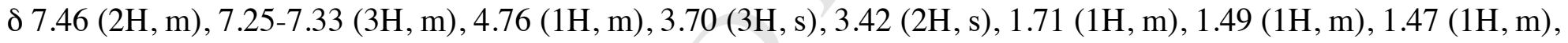
$0.98(3 \mathrm{H}, \mathrm{d}, \mathrm{J}=6.5 \mathrm{~Hz}), 0.95(3 \mathrm{H}, \mathrm{d}, \mathrm{J}=6.5 \mathrm{~Hz}) .{ }^{13} \mathrm{C} \mathrm{NMR}\left(125 \mathrm{MHz}, \mathrm{CDCl}_{3}\right) \delta$ 168.6, 152.2, 134.4, 131.9, 129.2, 127.8, 85.6, 80.4, 53.4, 37.9, 37.5, 24.9, 23.2, 21.2 ppm. IR (KBr) $\delta 3059,2959,1806,1749,1582,1439,1360,1257$, $1132,1048,963,744 \mathrm{~cm}^{-1}$.

syn-Methyl 5-cyclohexyl-2-oxo-4-((phenylthio)methyl)-1,3-dioxolane-4-carboxylate $6{ }^{1} \mathrm{H}$ NMR (500 MHz, $\left.\mathrm{CDCl}_{3}\right) \delta 7.39(2 \mathrm{H}, \mathrm{m}), 7.25-7.17(3 \mathrm{H}, \mathrm{m}), 4.37(1 \mathrm{H}, \mathrm{m}), 3.73(3 \mathrm{H}, \mathrm{s}), 3.57(1 \mathrm{H}, \mathrm{d}, \mathrm{J}=15.0 \mathrm{~Hz}), 3.35(1 \mathrm{H}, \mathrm{d}, \mathrm{J}=$ $15.0 \mathrm{~Hz}), 1.80(1 \mathrm{H}, \mathrm{m}), 1.40-1.77(5 \mathrm{H}, \mathrm{m}), 1.24-0.79(5 \mathrm{H}, \mathrm{m}) .{ }^{13} \mathrm{C}$ NMR $\left(125 \mathrm{MHz}, \mathrm{CDCl}_{3}\right) \delta 168.9,152.4,134.8$, $131.9,129.2,127.8,86.0,85.7,53.5,37.7,37.3,29.5,28.1,25.7,25.4,25.1$ ppm. IR (KBr) $\delta 3060,2929,2857,1741$, $1582,1402,1195,1024,927,845,713,629 \mathrm{~cm}^{-1}$. HRMS $\mathrm{m} / z$ calcd. for $\mathrm{C}_{18} \mathrm{H}_{22} \mathrm{O}_{5} \mathrm{SNa}\left[\mathrm{M}+\mathrm{Na}^{+}\right]: 373.1086$, found: 373.1089.

anti-Methyl 5-cyclohexyl-2-oxo-4-((phenylthio)methyl)-1,3-dioxolane-4-carboxylate $7 .{ }^{1} \mathrm{H}$ NMR (500 MHz, $\left.\mathrm{CDCl}_{3}\right) \delta 7.47(2 \mathrm{H}, \mathrm{m}), 7.24-7.33(3 \mathrm{H}, \mathrm{m}), 4.43(1 \mathrm{H}, \mathrm{m}), 3.76(3 \mathrm{H}, \mathrm{s}), 3.56(1 \mathrm{H}, \mathrm{d}, \mathrm{J}=15.0 \mathrm{~Hz}), 3.48(1 \mathrm{H}, \mathrm{d}, \mathrm{J}=$ 
15.0Hz), $1.77(1 \mathrm{H}, \mathrm{m}), 1.68-1.76(5 \mathrm{H}, \mathrm{m}), 1.10-1.25(5 \mathrm{H}, \mathrm{m}) .{ }^{13} \mathrm{C}$ NMR $\left(125 \mathrm{MHz}, \mathrm{CDCl}_{3}\right) \delta 167.9,152.6,134.7$,

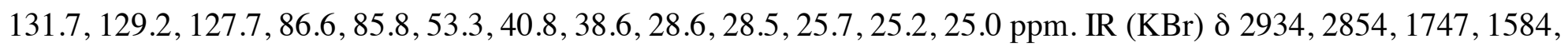
$1584,1440,1178,1052,930,634 \mathrm{~cm}^{-1}$.

\section{Acknowledgements}

This work was financed by Bancaixa-UJI foundation (P1 1B2011-59 and P1 1B2011-28). The authors also are grateful to the Serveis Centrals d'Instrumentació Científica (SCIC) of the Universitat Jaume I for providing us with mass spectrometry and NMR.

\section{References and Footnotes}

1- Chong, J. M.; Sharpless, K. B. Tetrahedron Lett. 1985, 26, 4683-4686.

2- Meth-Cohn, O.; Moore, C.; Taljaard, H. C. J. Chem. Soc., Perkin Trans. 1 1988, 2663-2674.

3- Saito, S.; Takahashi, N.; Ishikawa, T.; Moriwake, T. Tetrahedron Lett. 1991, 32, 667-670.

4- Lanier, M.; Pastor, R. Tetrahedron Lett. 1995, 36, 2491-2492.

5- Righi, G.; Rumboldt, G.; Bonini, C. J. Org. Chem. 1996, 61, 3557-3560.

6- Concellón, J. M.; Bardales, E. Org. Lett. 2002, 4, 189-191.

7- Concellón, J. M.; Bardales, E.; Llavona, R. J. Org. Chem. 2003, 68, 1585-1588 and refs cited therein.

8- Rodríguez, S.; Izquierdo, F.; López, I.; González, F. V. Tetrahedron 2006, 62, 11112-11123.

9- López, I.; Izquierdo, J.; Rodríguez, S.; González, F. V. J. Org. Chem. 2007, 72, 6614-6617.

10- Bailey, M.; Markó, I. E.; Ollis, W. D.; Rasmussen, P. R. Tetrahedron Lett. 1990, 31 , 4509-4512.

11- Bailey, M.; Staton, I.; Ashton, P. R.; Markó, I. E.; Ollis, W. D. Tetrahedron: Asymm. 1991, 2, 495-509.

12- Svenda, M.; Myers, A. G. Org. Lett. 2009, 11, 2437-2440.

13- Yu, C.; Liu, B.; Hu, L. J. Org. Chem. 2001, 66, 5413-5418.

14- García-Ruano, J. L.; Fajardo, C.; Fraile, A.; Martí, M. R. J. Org. Chem. 2005, 70, 4300-4306. 
15- Adam, W.; Braun, M.; Griesbek, A.; Lucchini, V.; Staab, E.; Will, B. J. Am. Chem. Soc. 1989, 111, $203-212$. 16- Lee, K.; Loh, T. Chem. Commun. 2006, 40, 4209-4211.

17- Brzezinski, L. J.; Rafel, S.; Leahy, J. W. Tetrahedron 1997, 53, 16423-16434.

18- Mi, X.; Luo, S.; Cheng, J. J. Org. Chem. 2005, 70, 2338-2341.

19- Aggarwal, V. A.; Emme, I.; Fulford, S. Y. J. Org. Chem. 2003, 68, 692-700.

20- Guo, Y.; Shao, G.; Li, L.; Wu, W.; Li, R.; Li, J.; Song, J.; Qiu, L.; Prashad, M.; Kwong, F. Y. Adv. Synth. Cat. 2010, 352, 1539-1553. 


\section{Supporting Information}

Study of the Stereoselectivity of the Nucleophilic Epoxidation of 3Hydroxy-2-methylene Esters

Antonio Latorre, José A. Sáez, Santiago Rodríguez, Florenci V. González*

Departament de Química Inorgànica i Orgànica, Universitat Jaume I, 12080 Castelló, Spain 


\section{Contents}

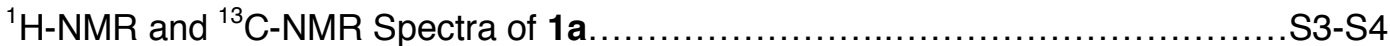

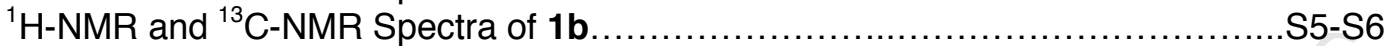

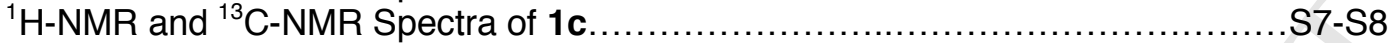

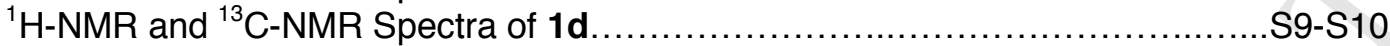

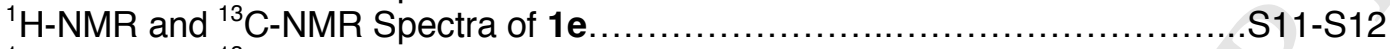

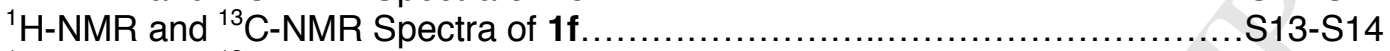

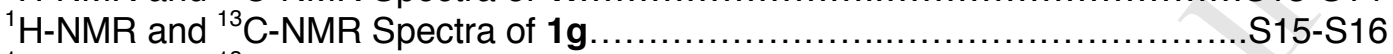

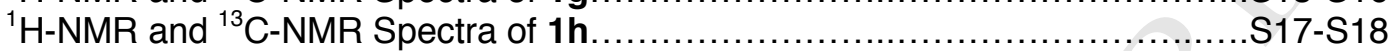

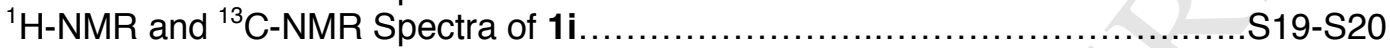

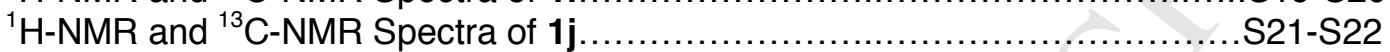

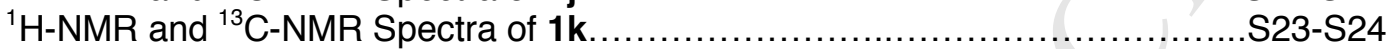

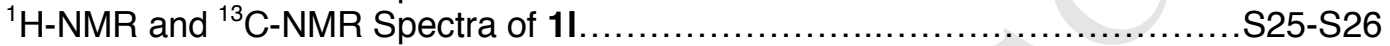

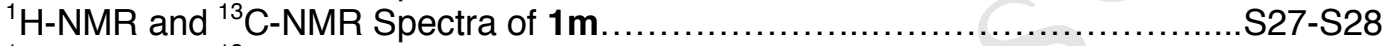

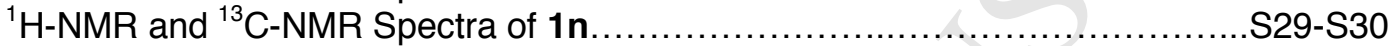

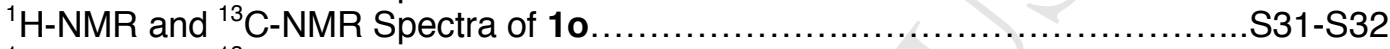

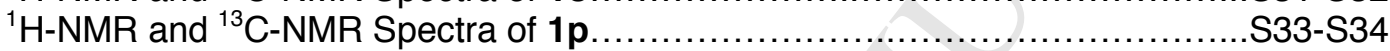

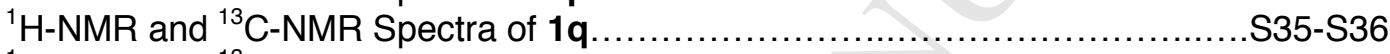

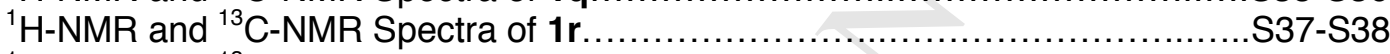

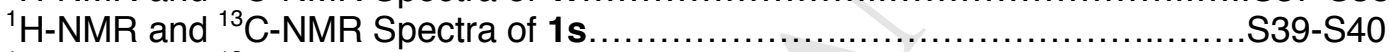

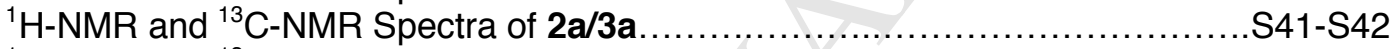

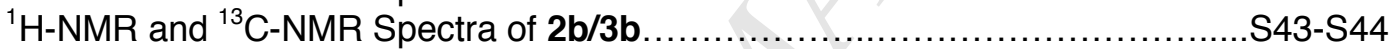

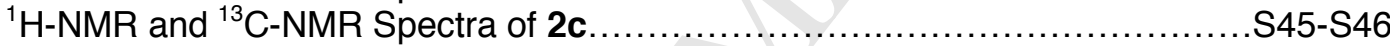

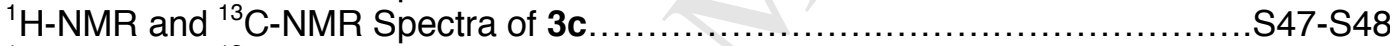

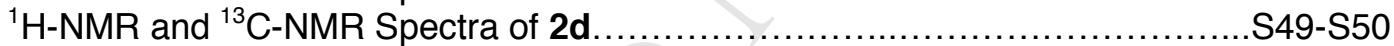

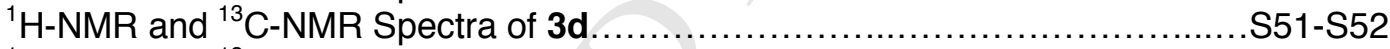

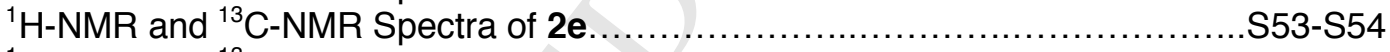

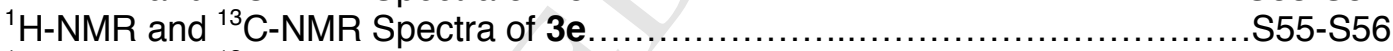

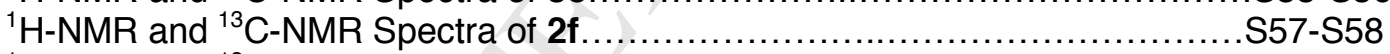

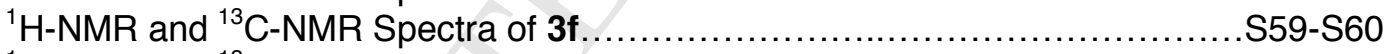

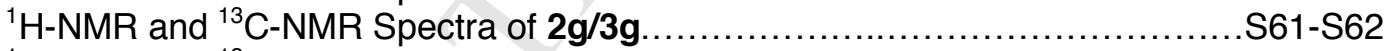

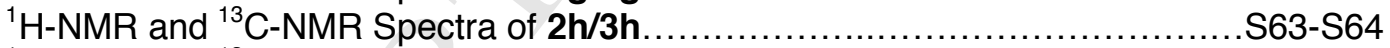

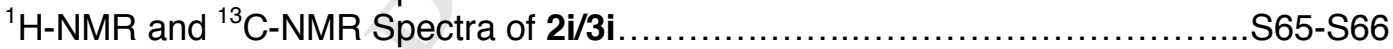

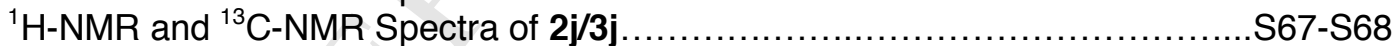

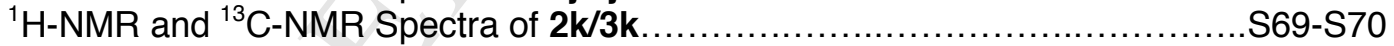

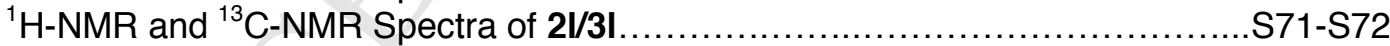

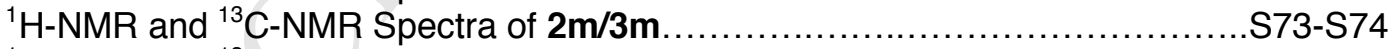

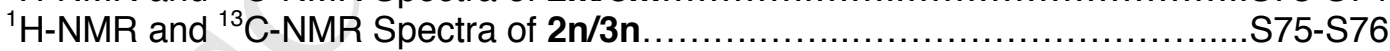

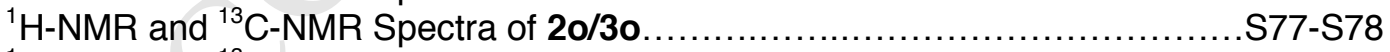

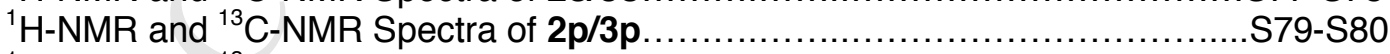

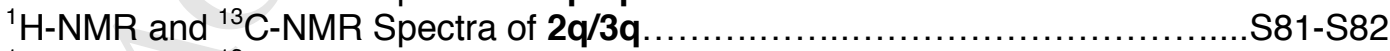

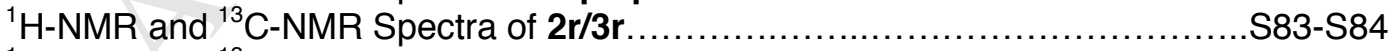

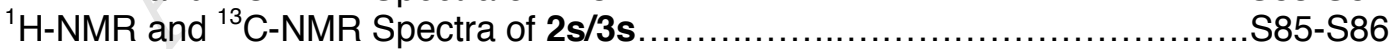

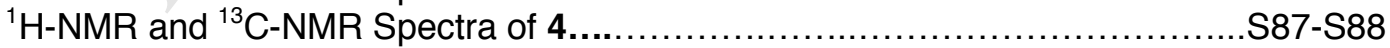

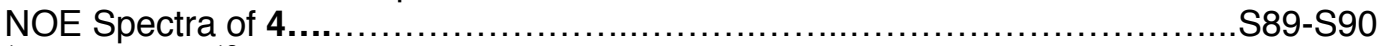

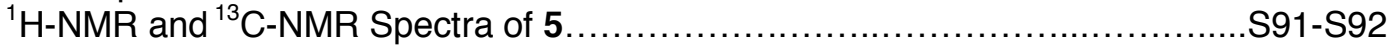

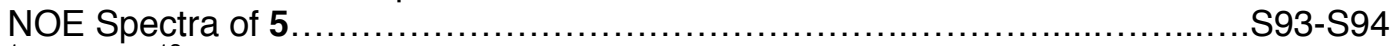

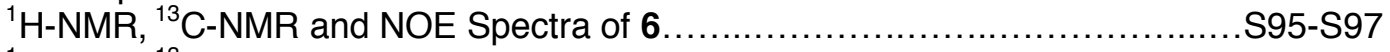

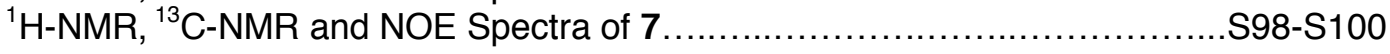




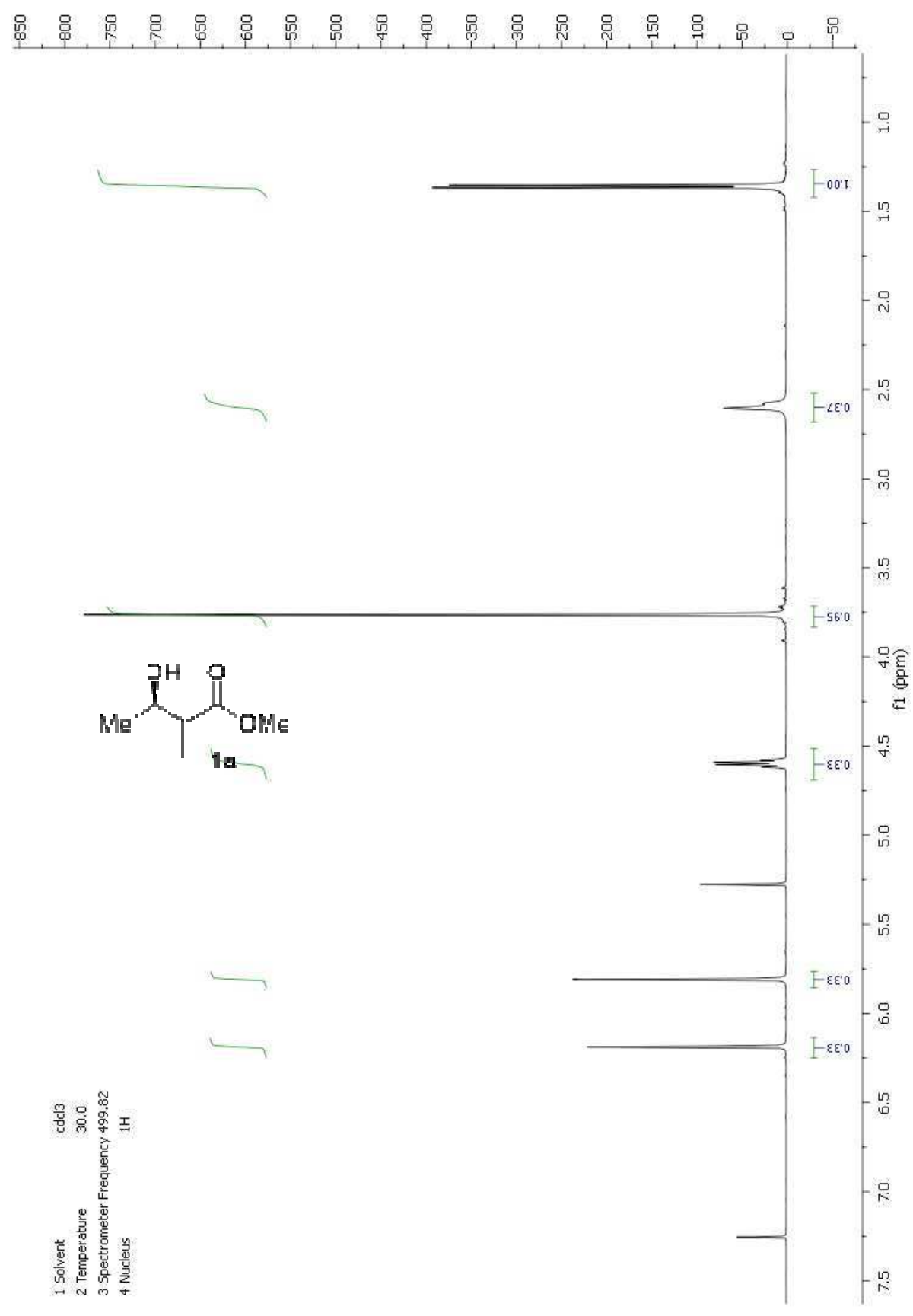




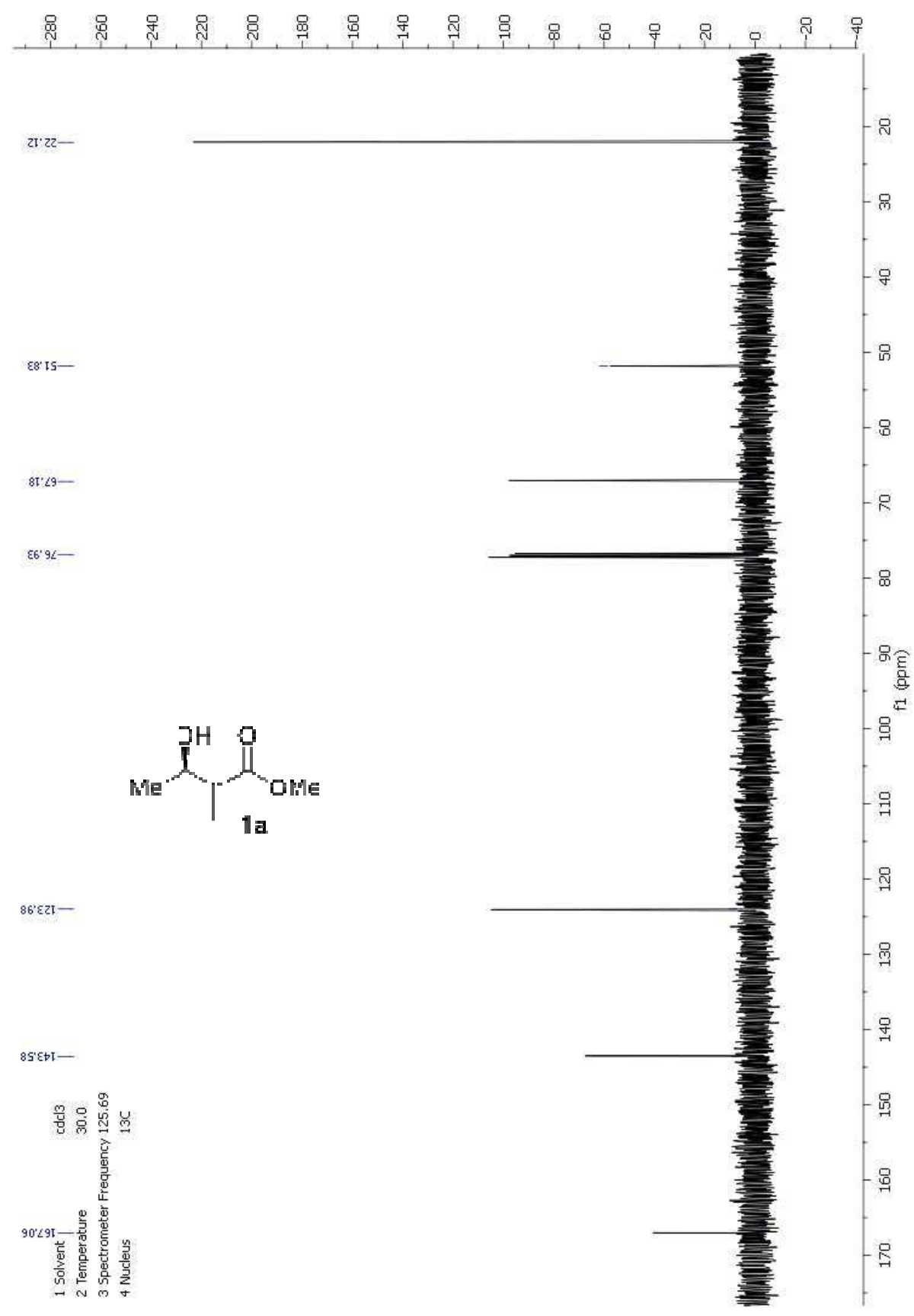




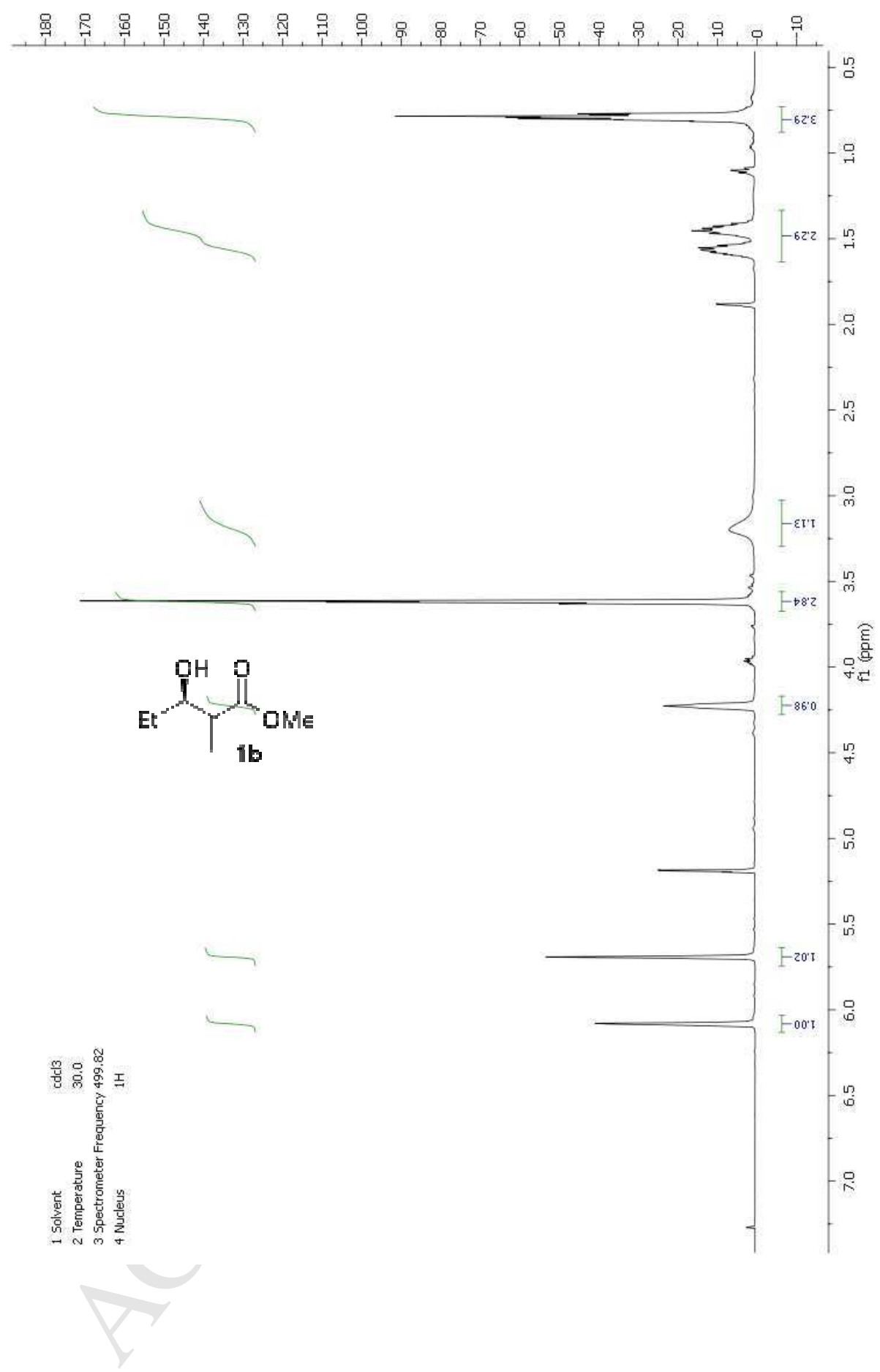




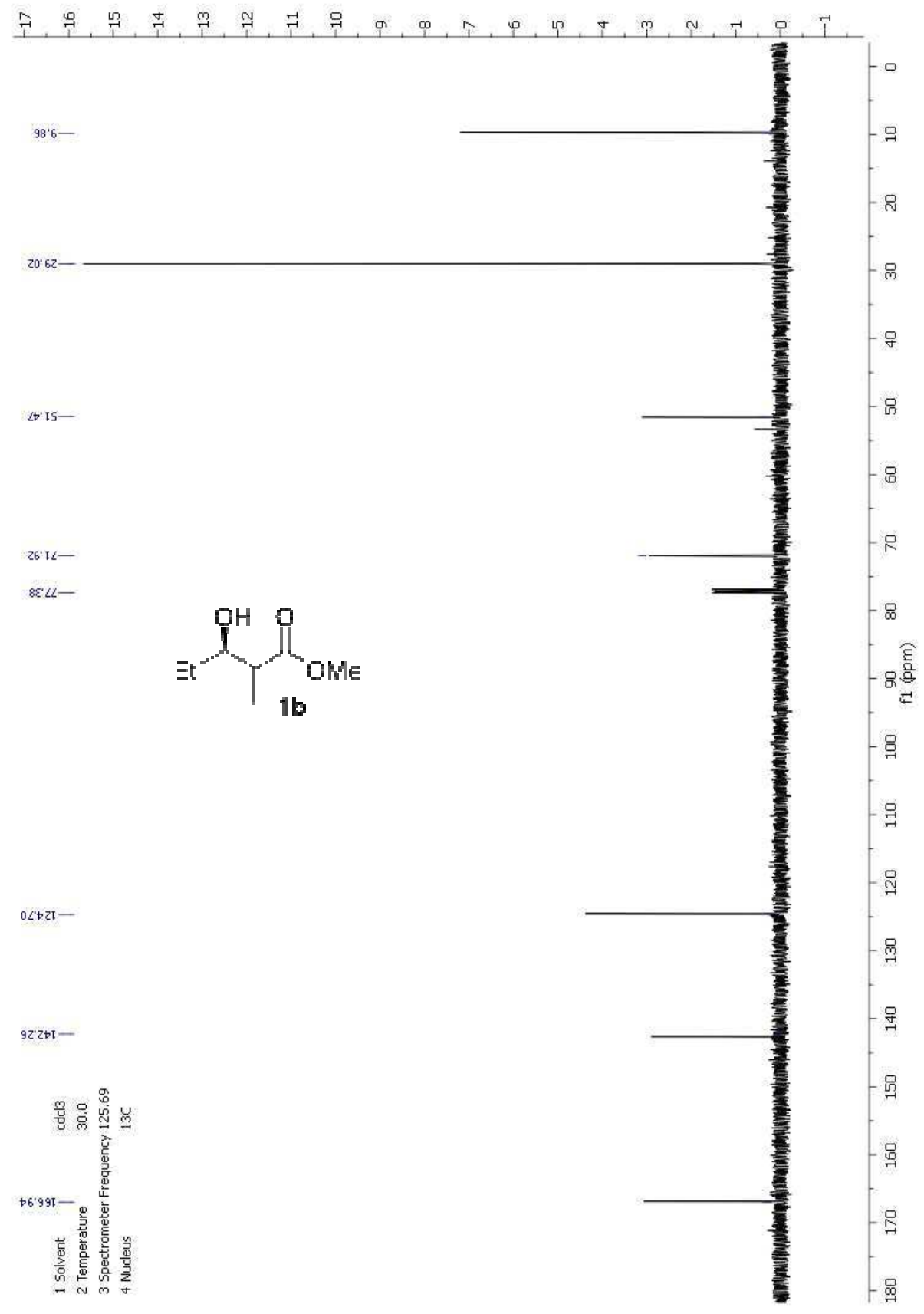




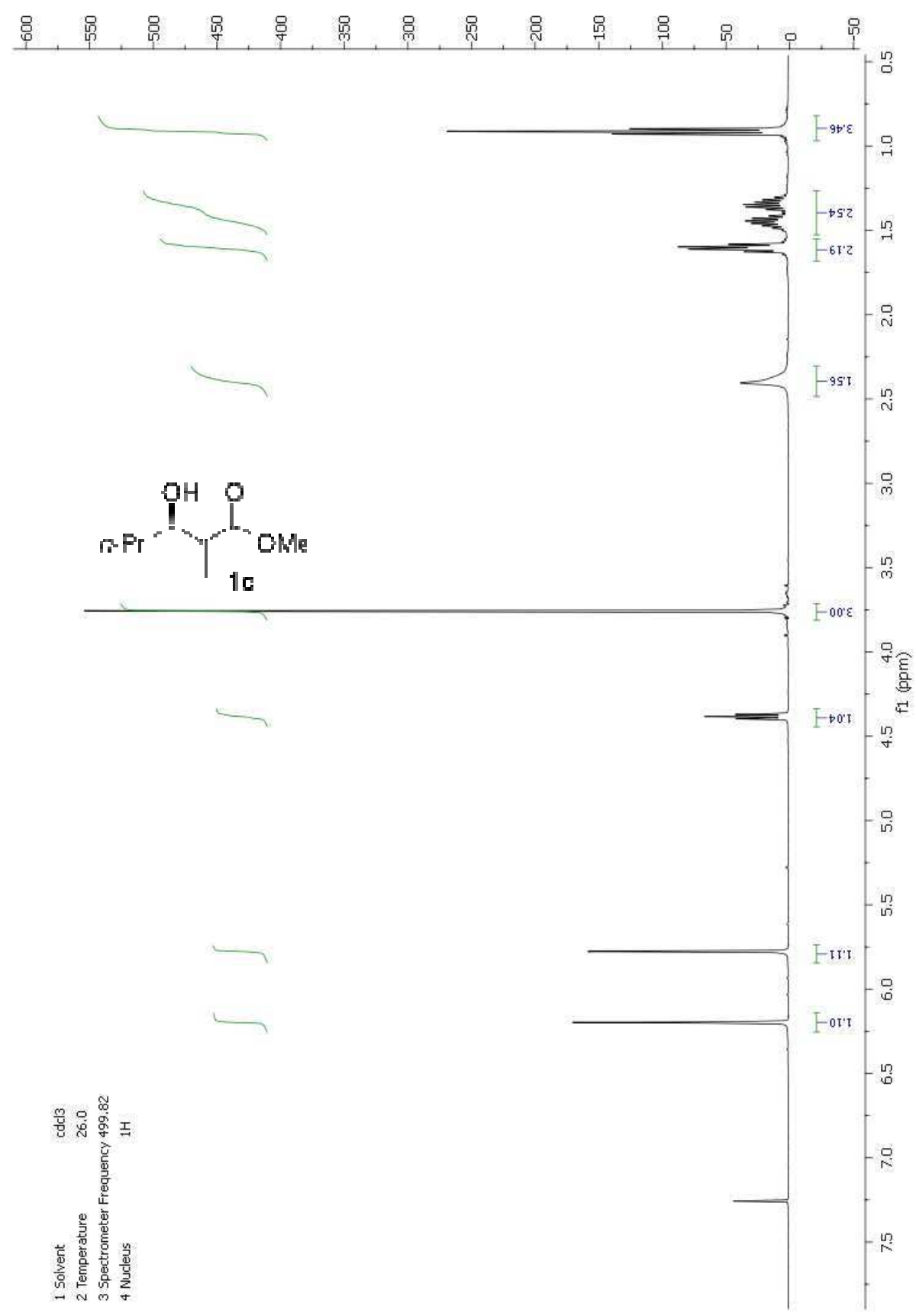




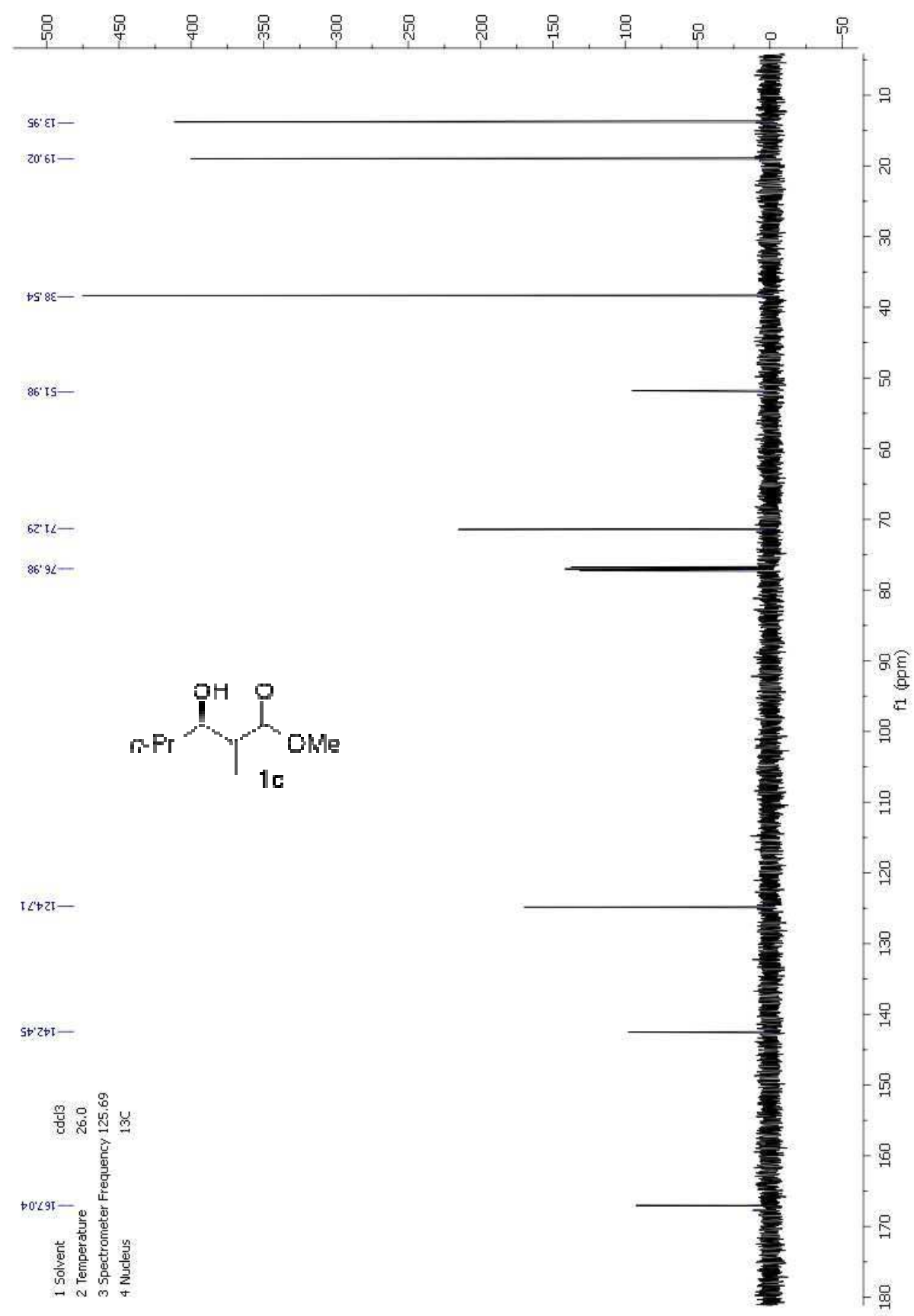




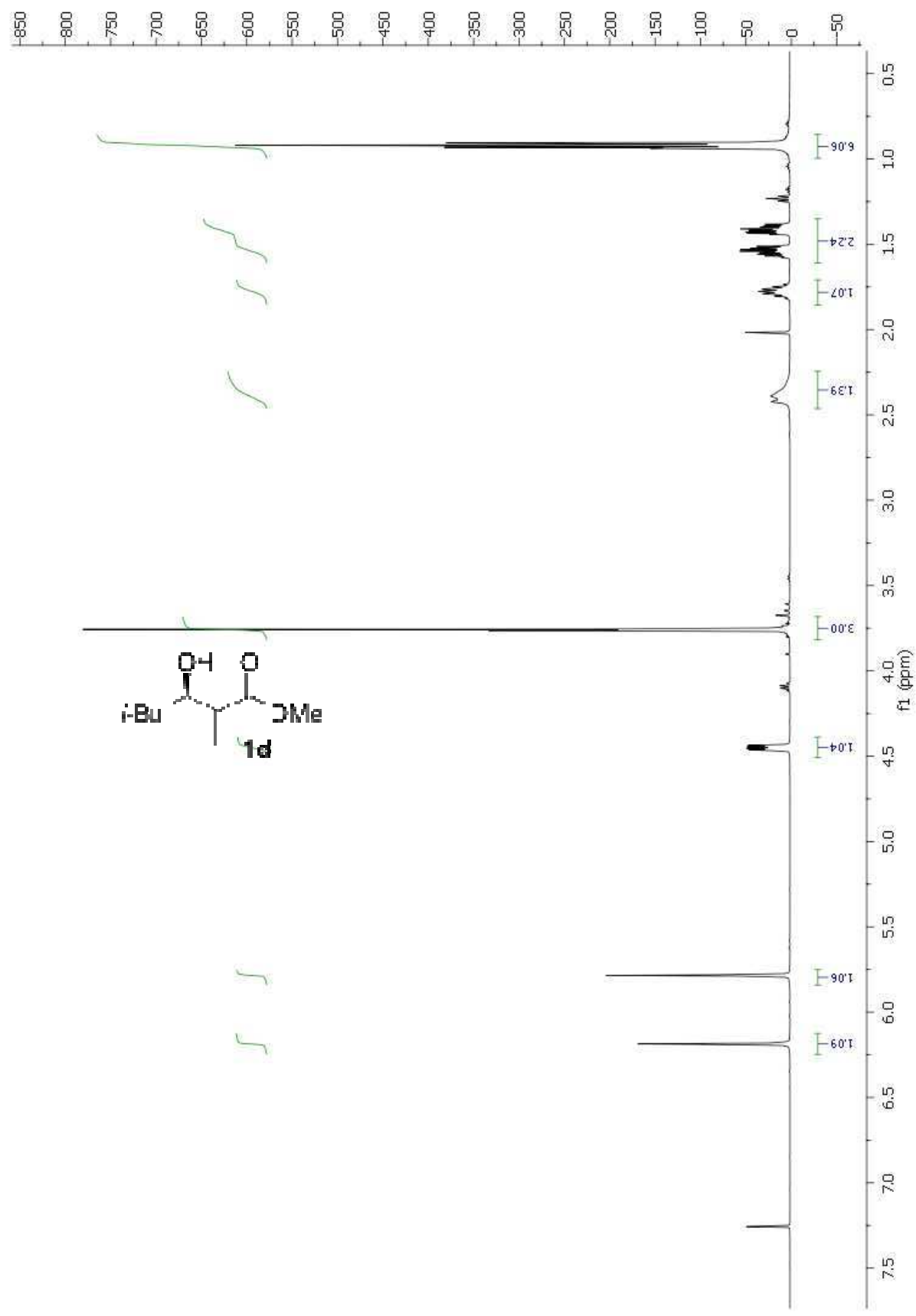




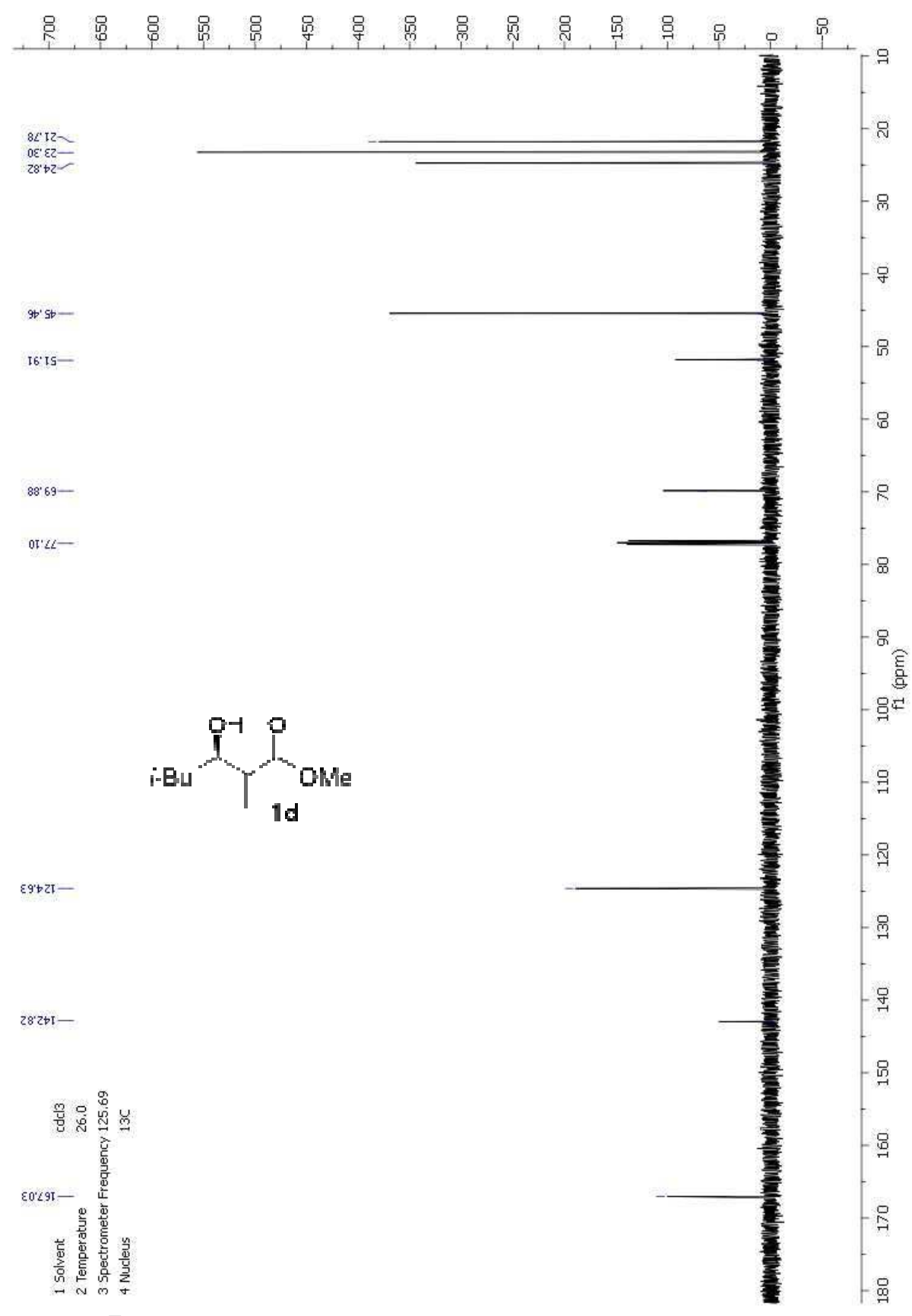




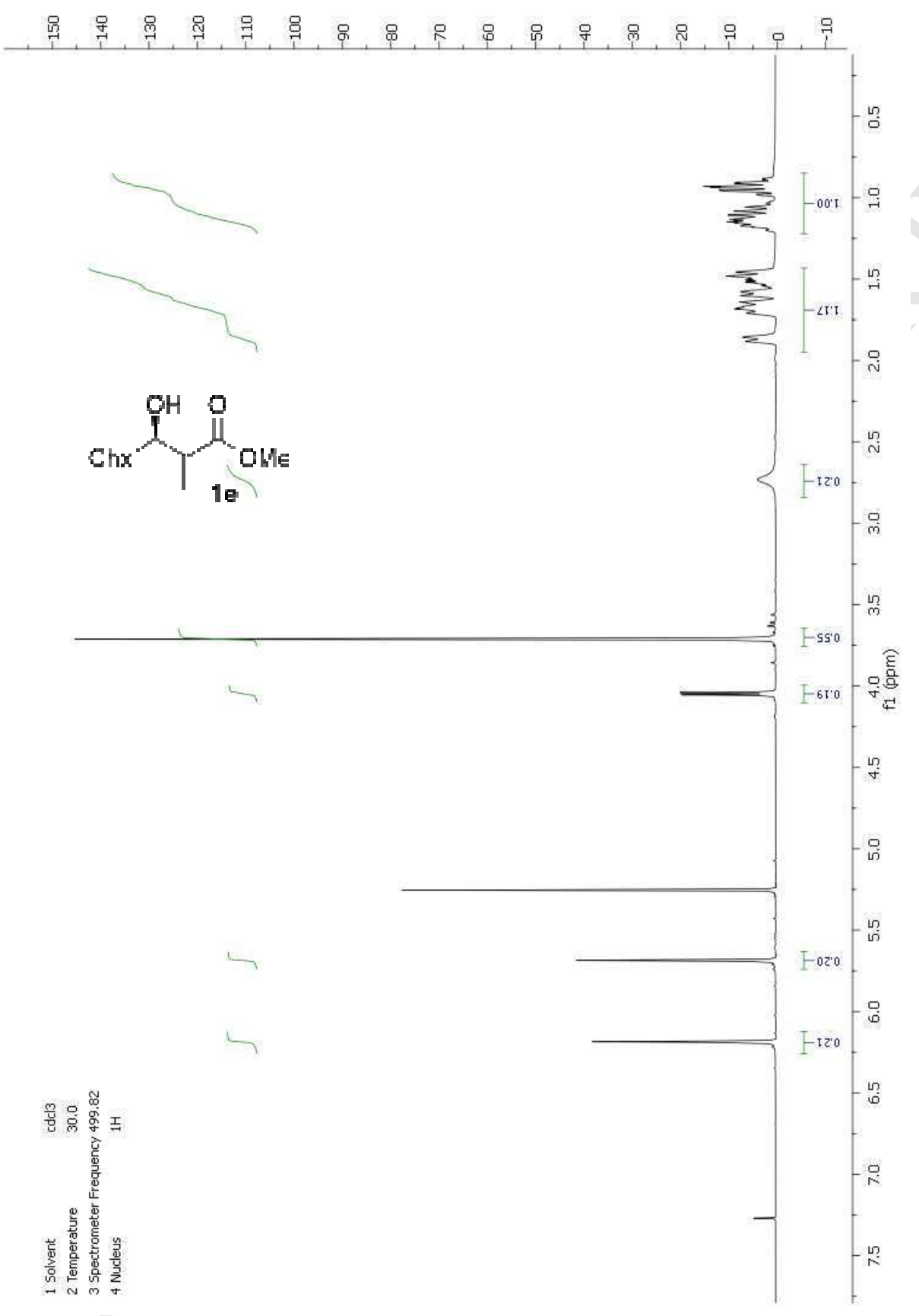




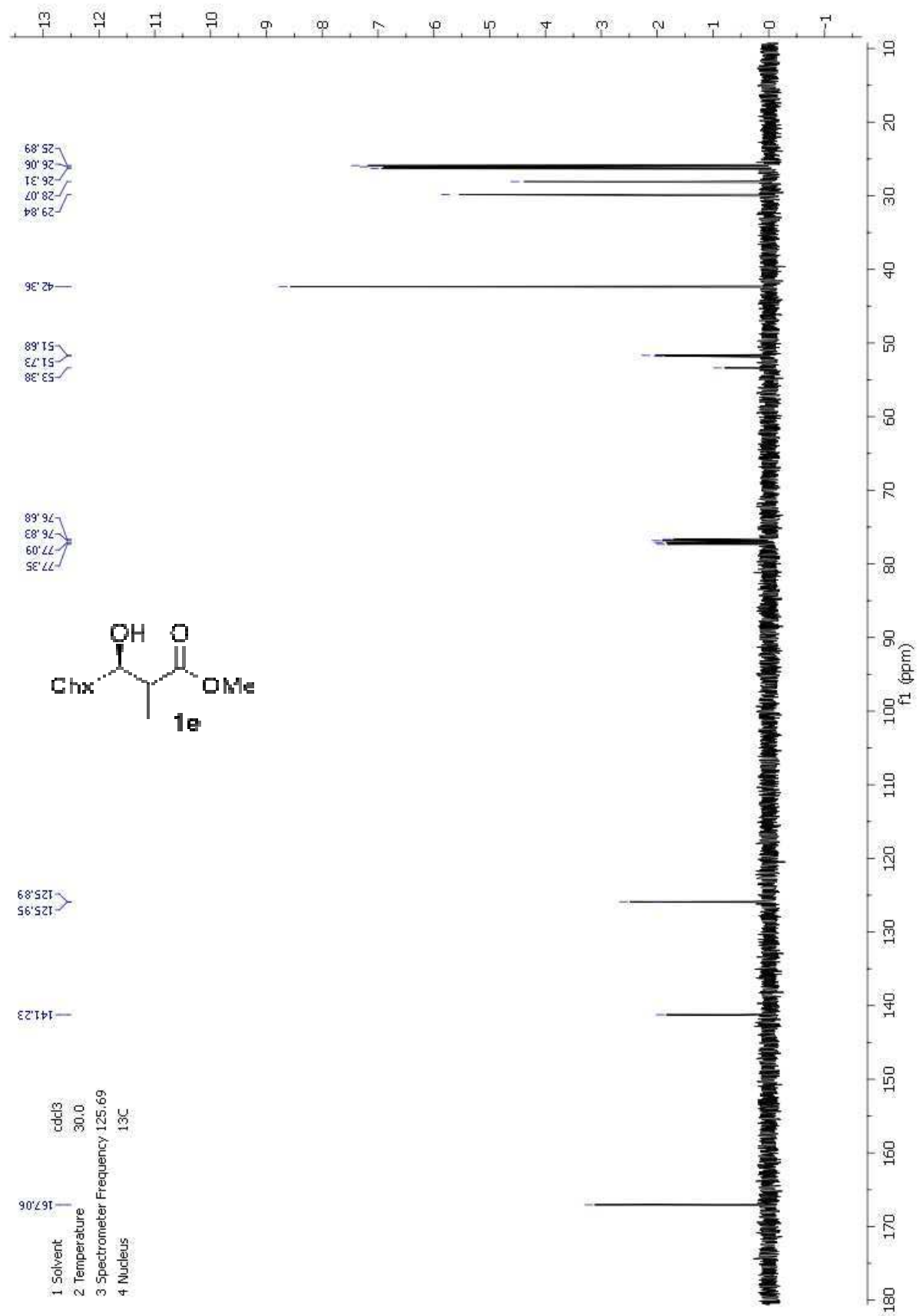




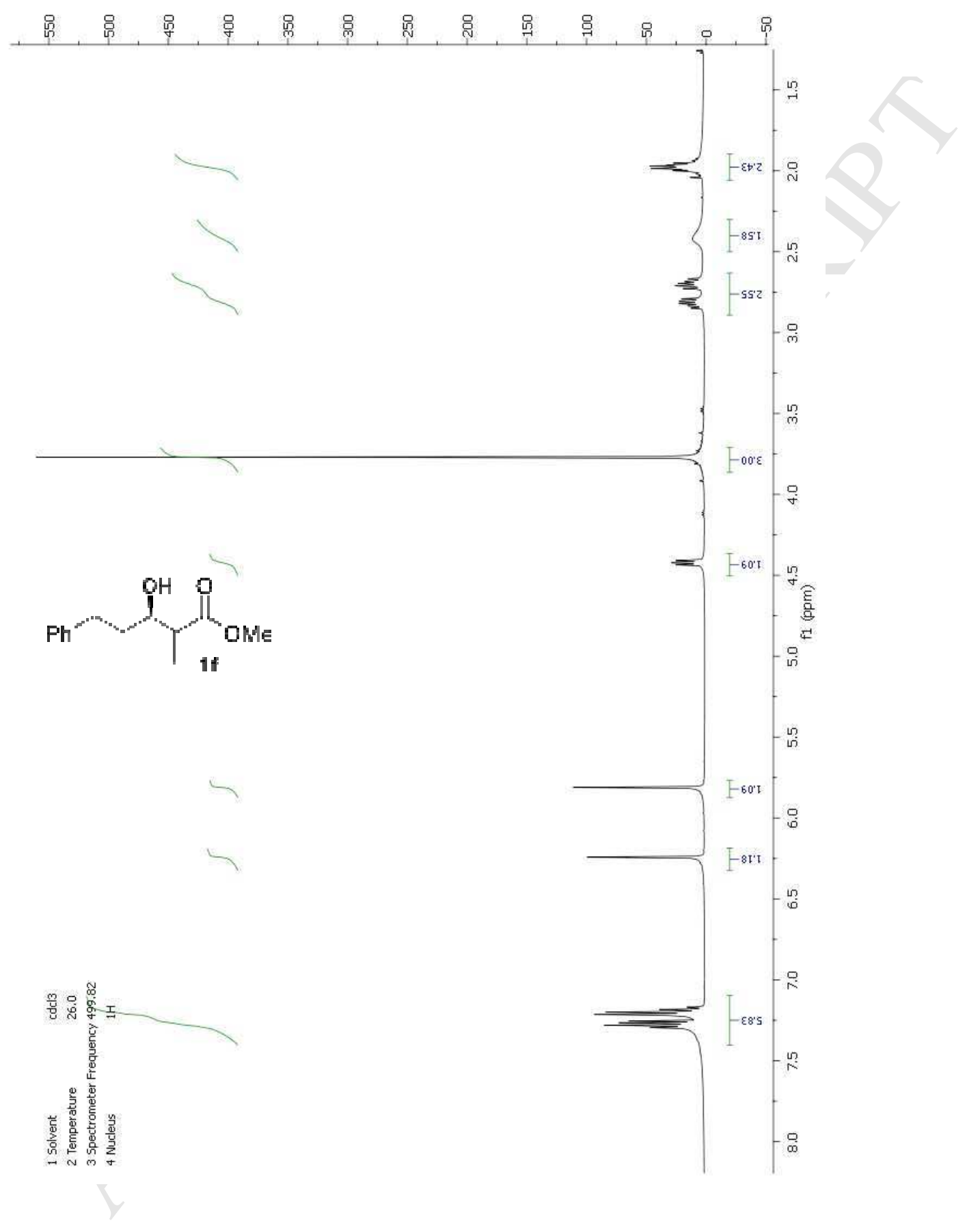




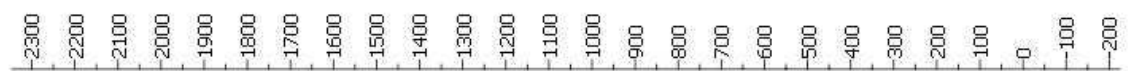

हr. $02-$<smiles>COC(=O)C(C)[C@H](O)CCc1ccccc1</smiles>

$\left.\right|_{-0} ^{1}$

$20^{\circ} z \varepsilon-$

เ6 $2 \varepsilon-$

28 T5-

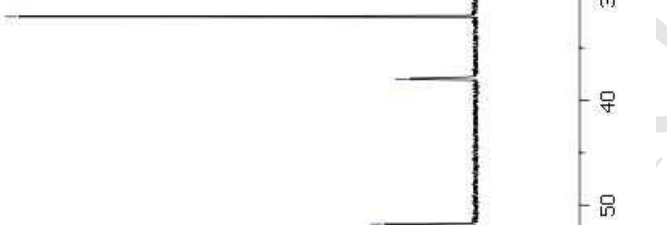

TIf $_{11}$

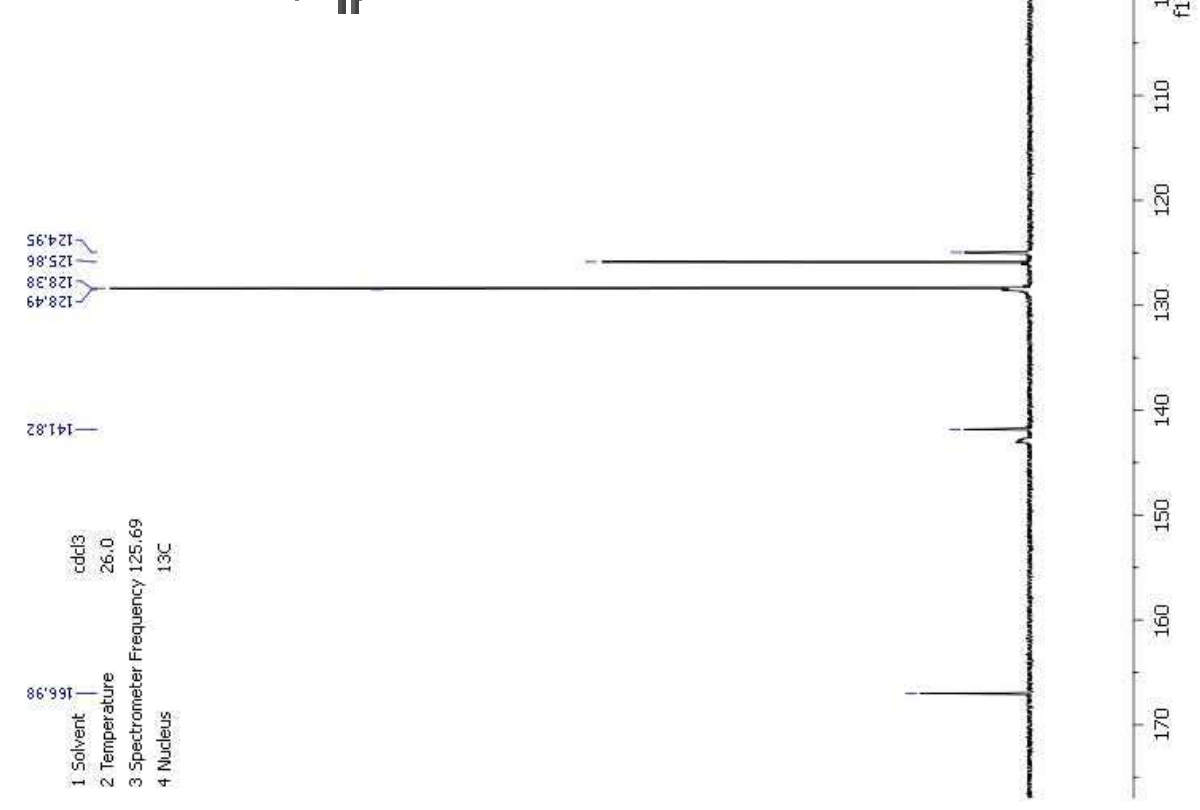




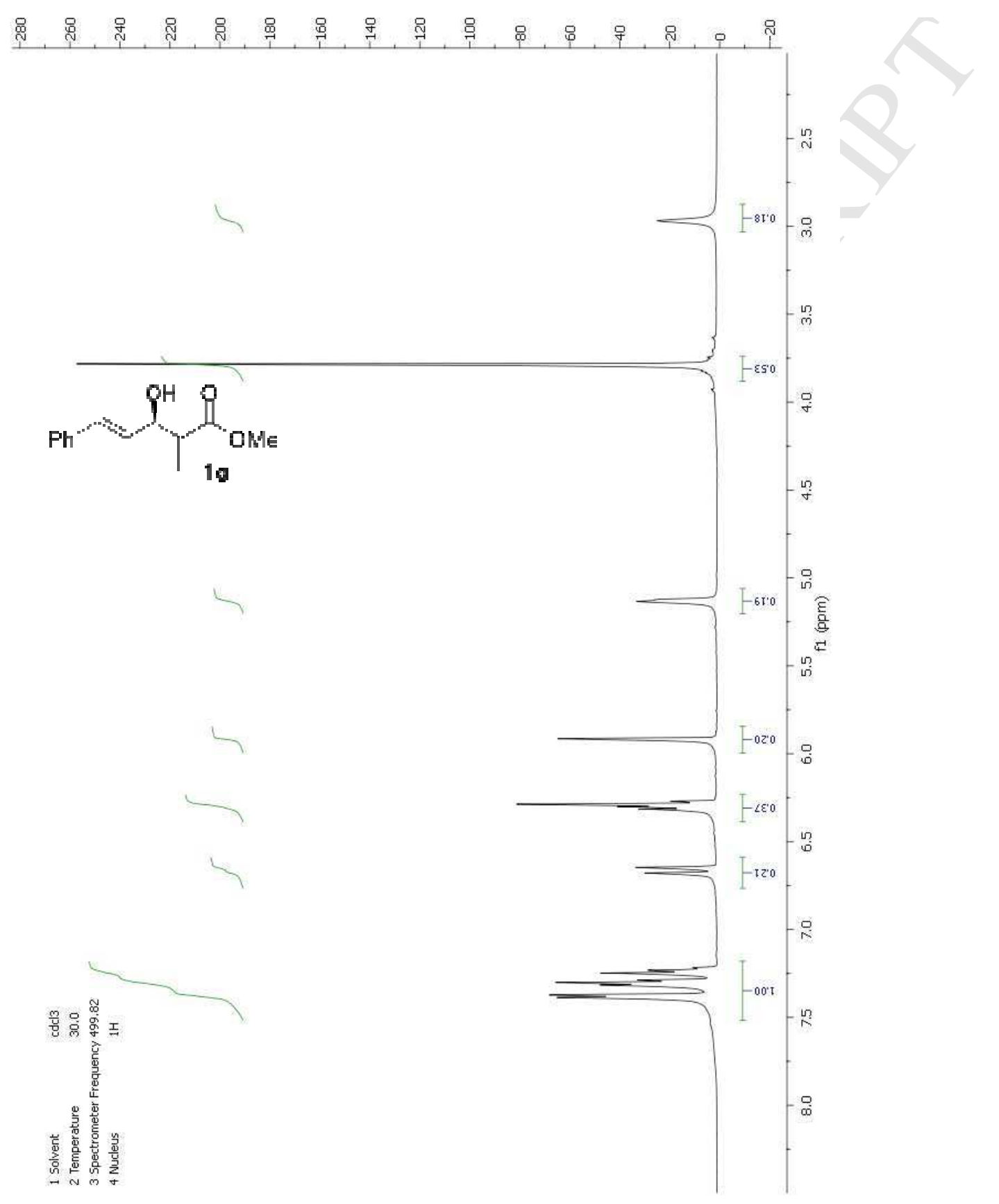




$$
\text { a }
$$




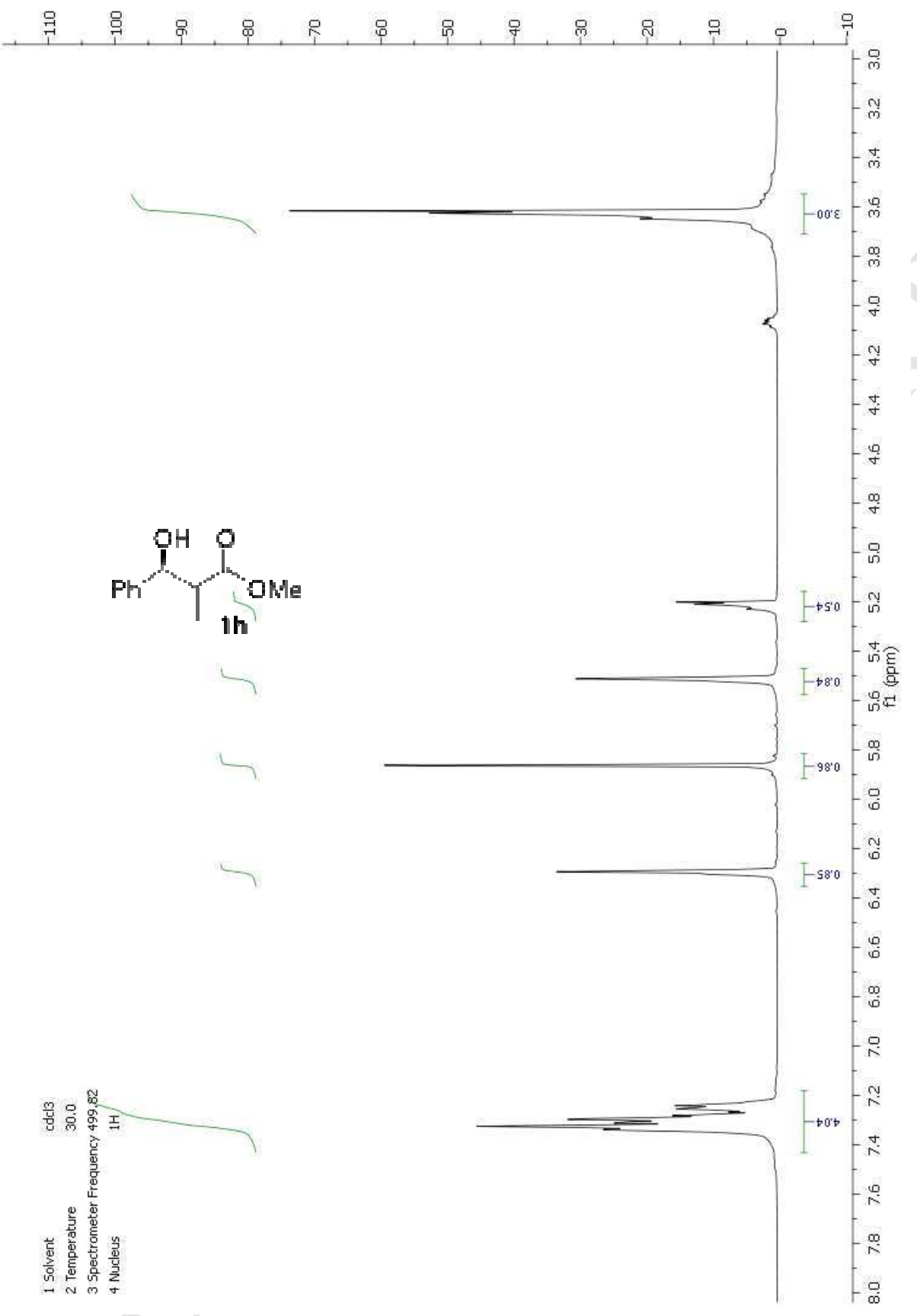




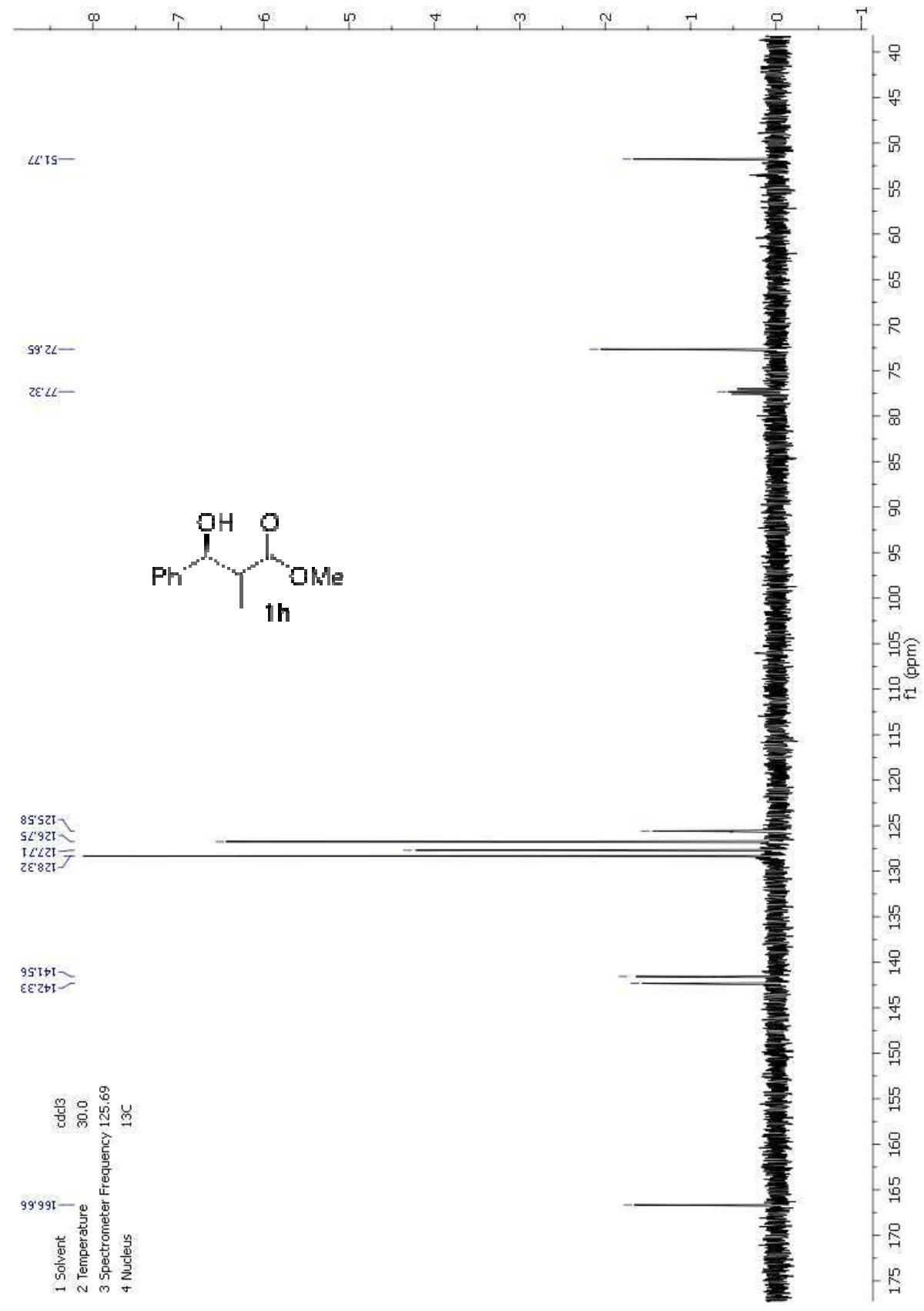




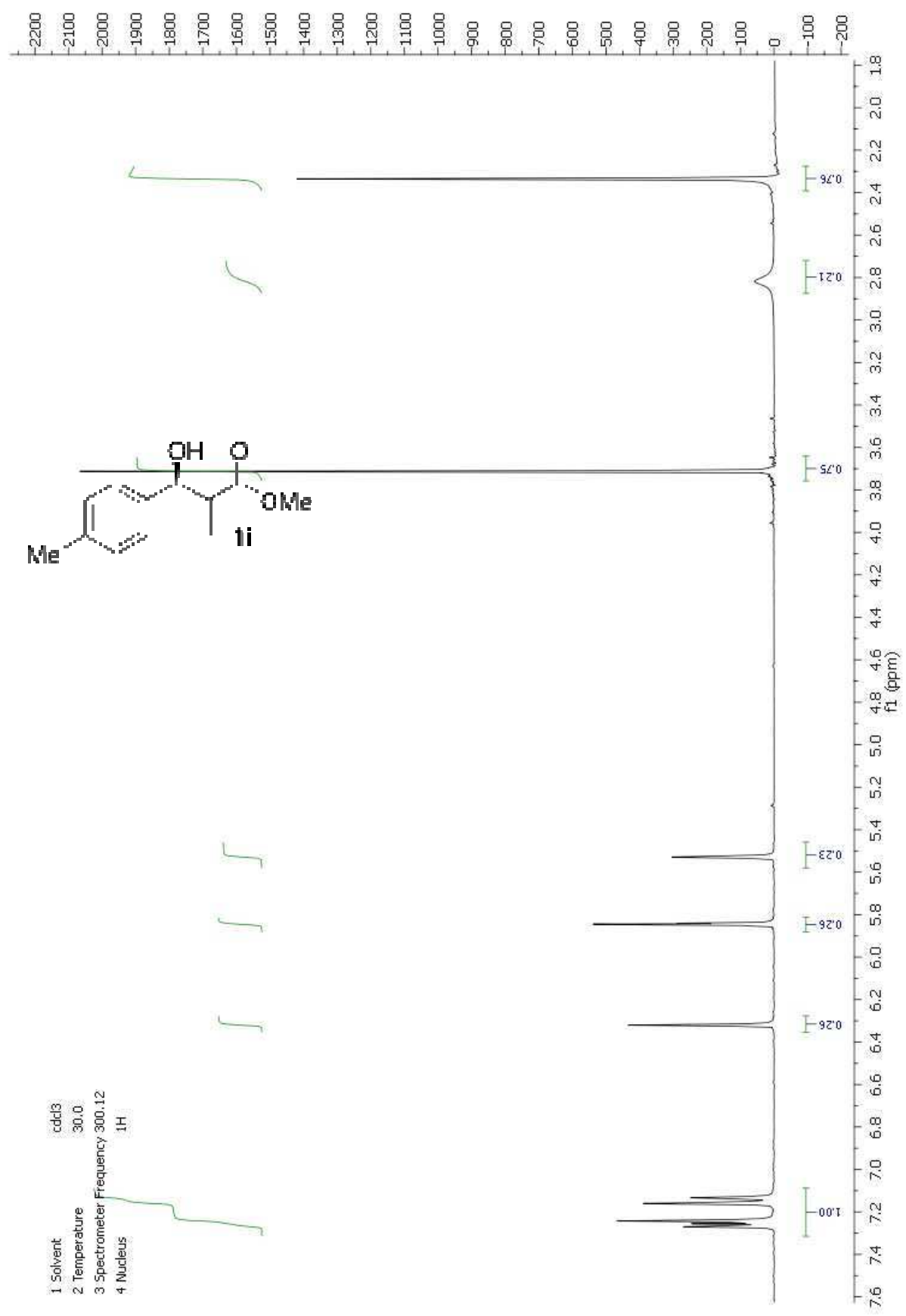




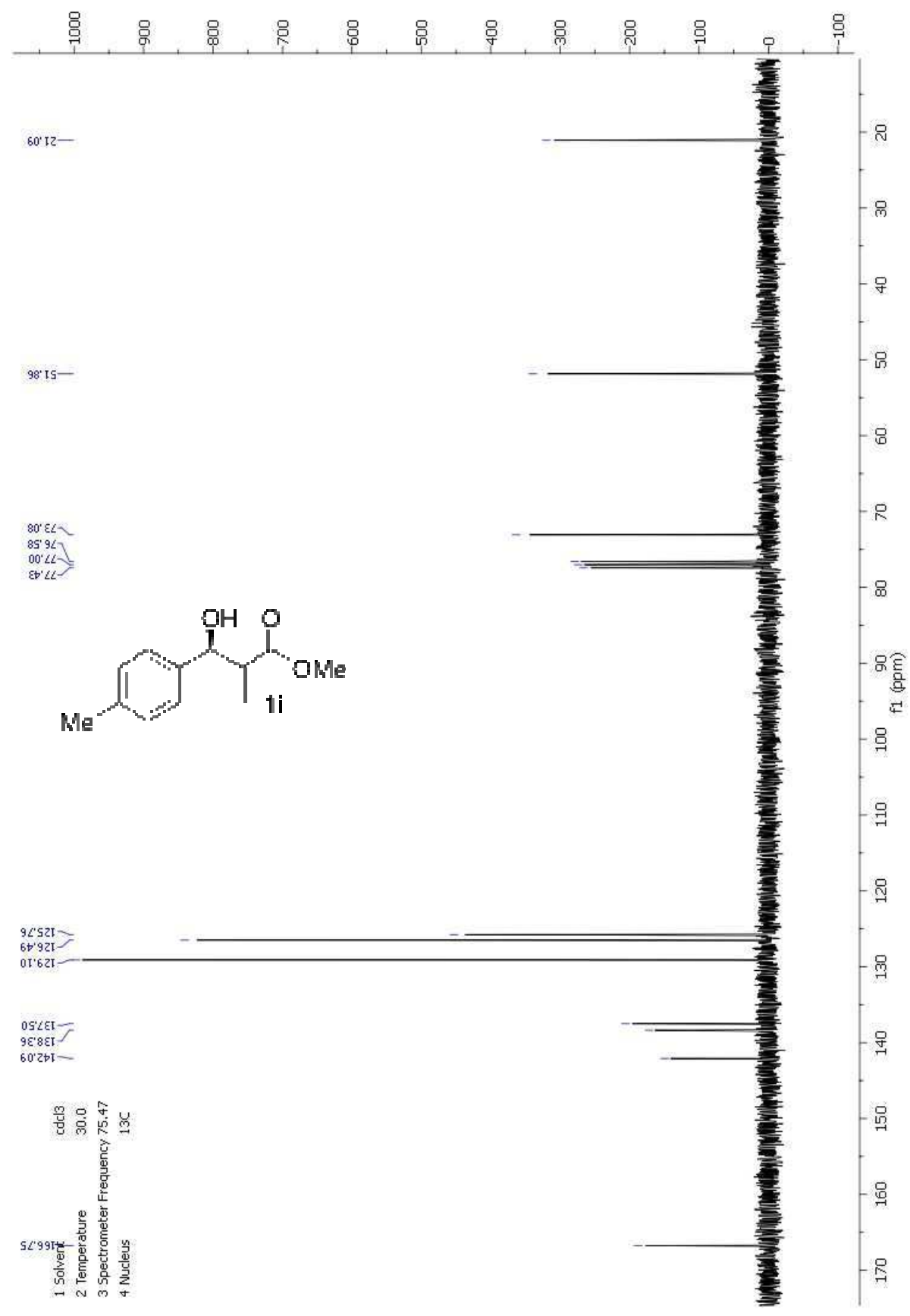




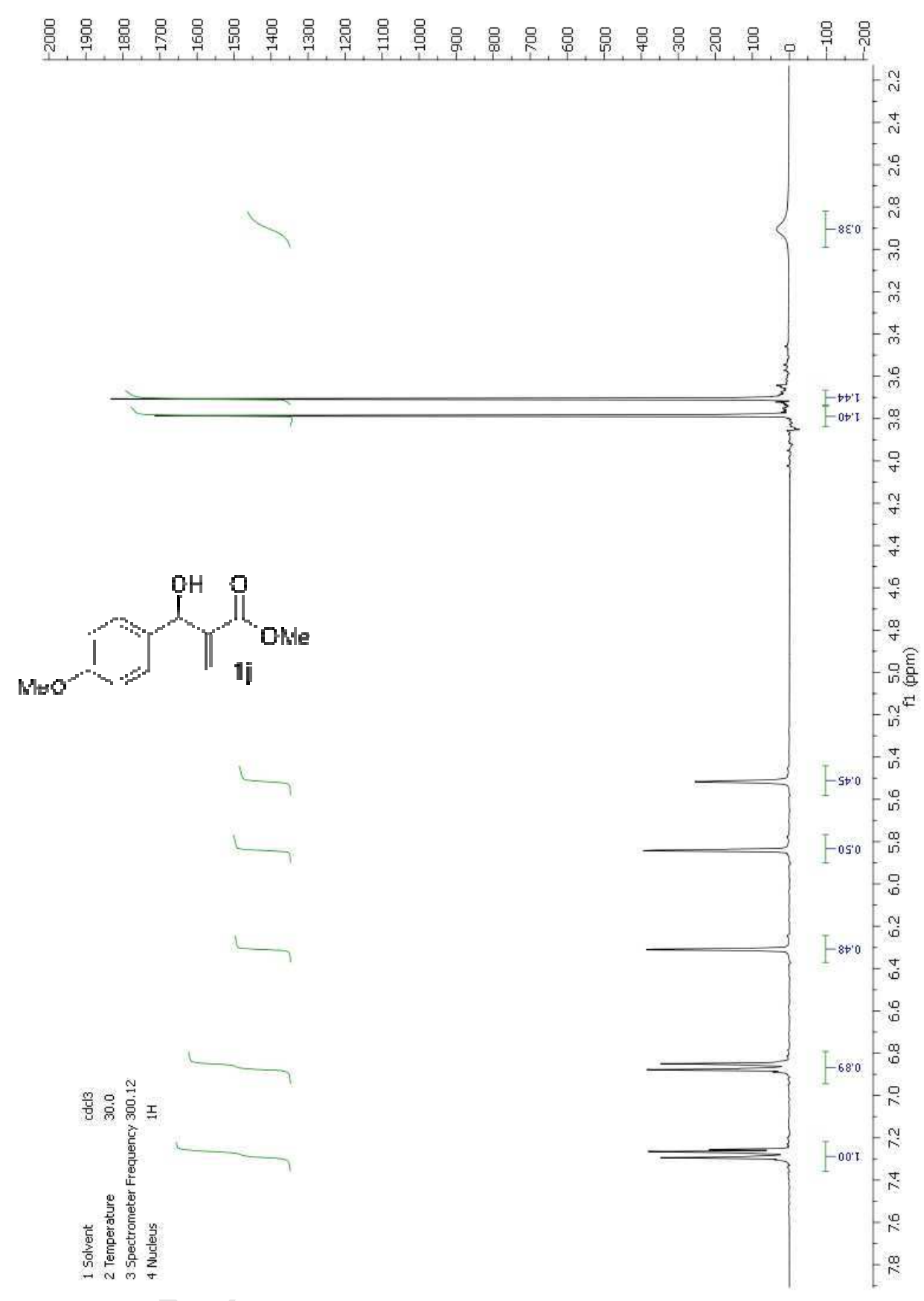




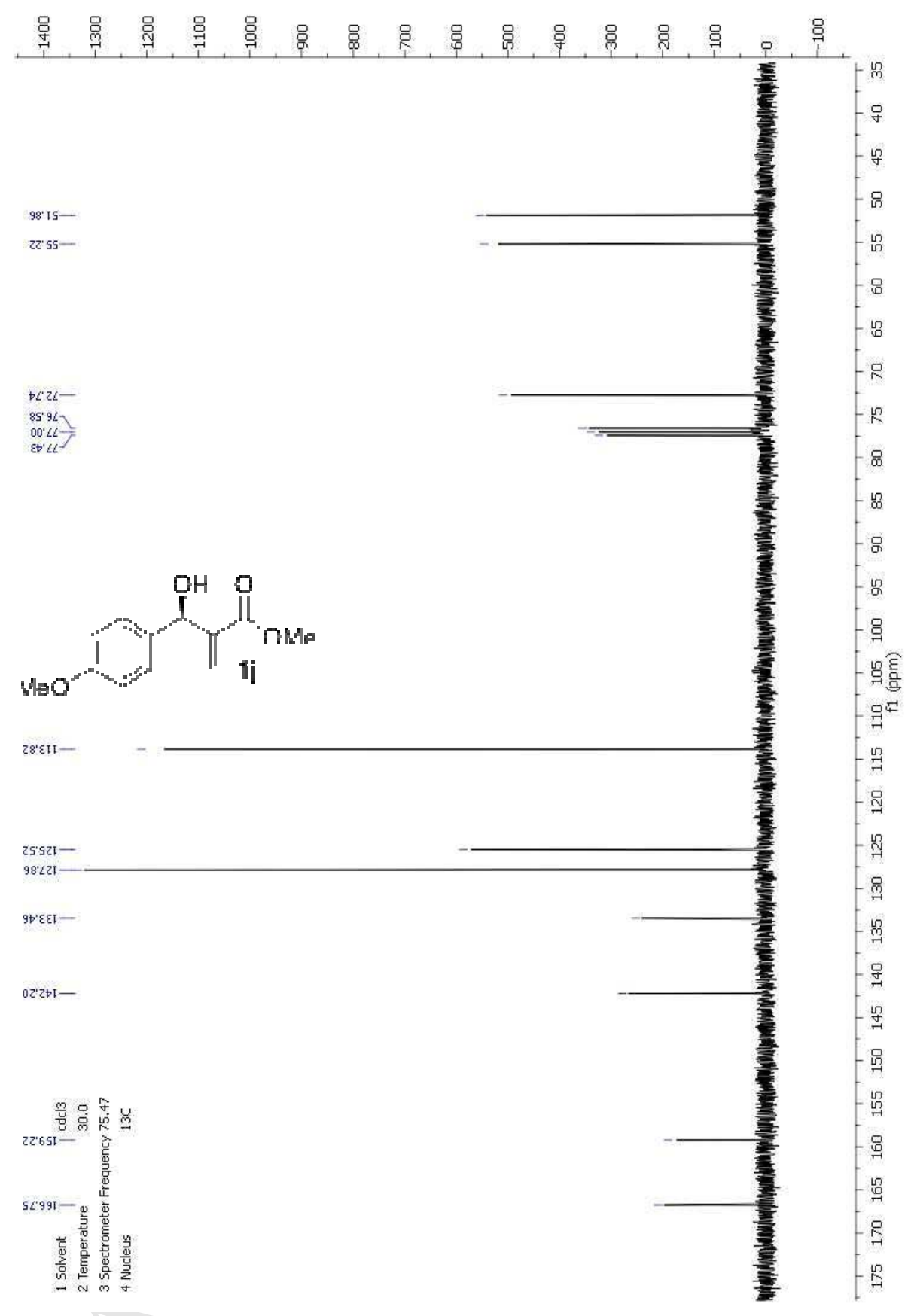




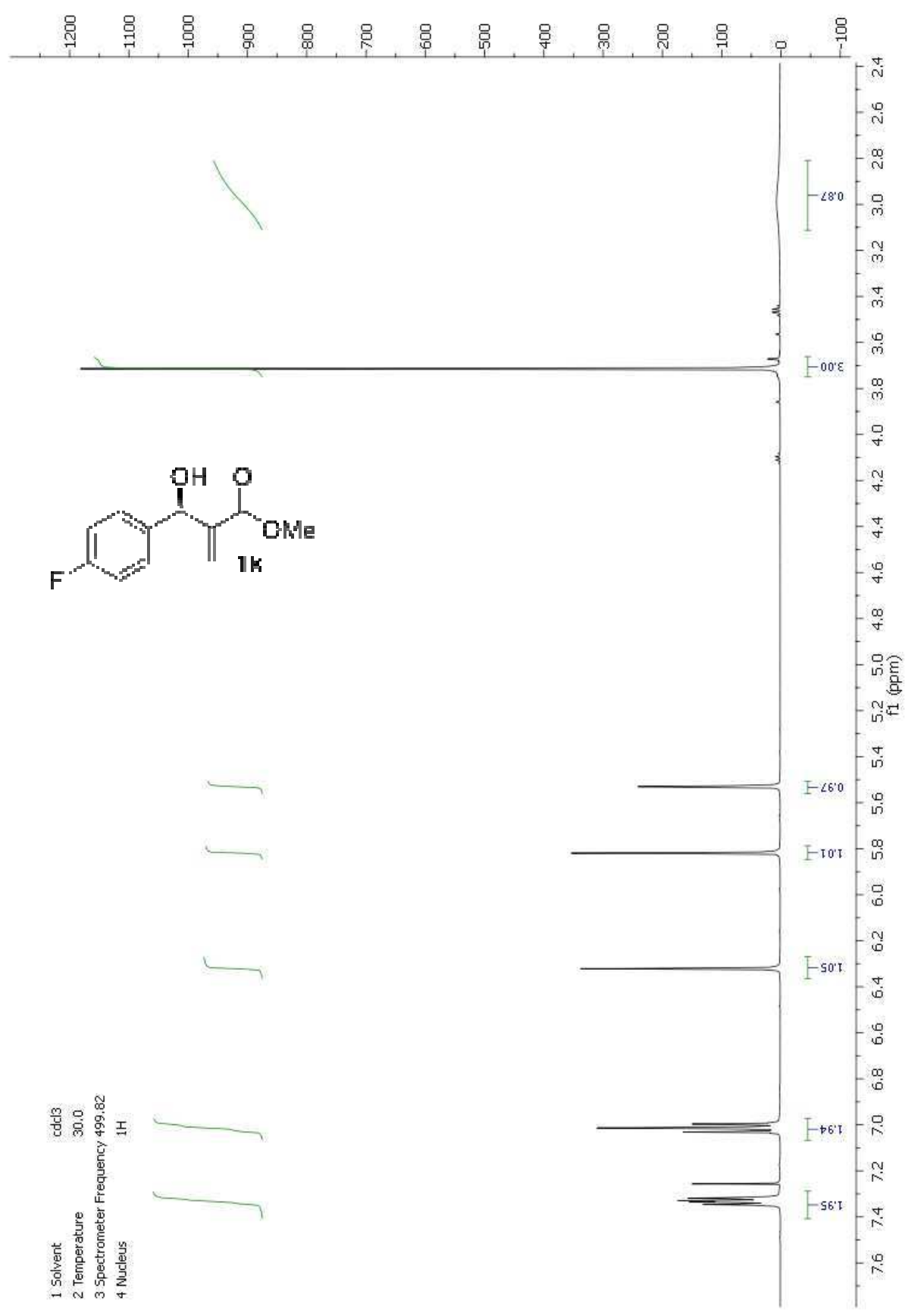




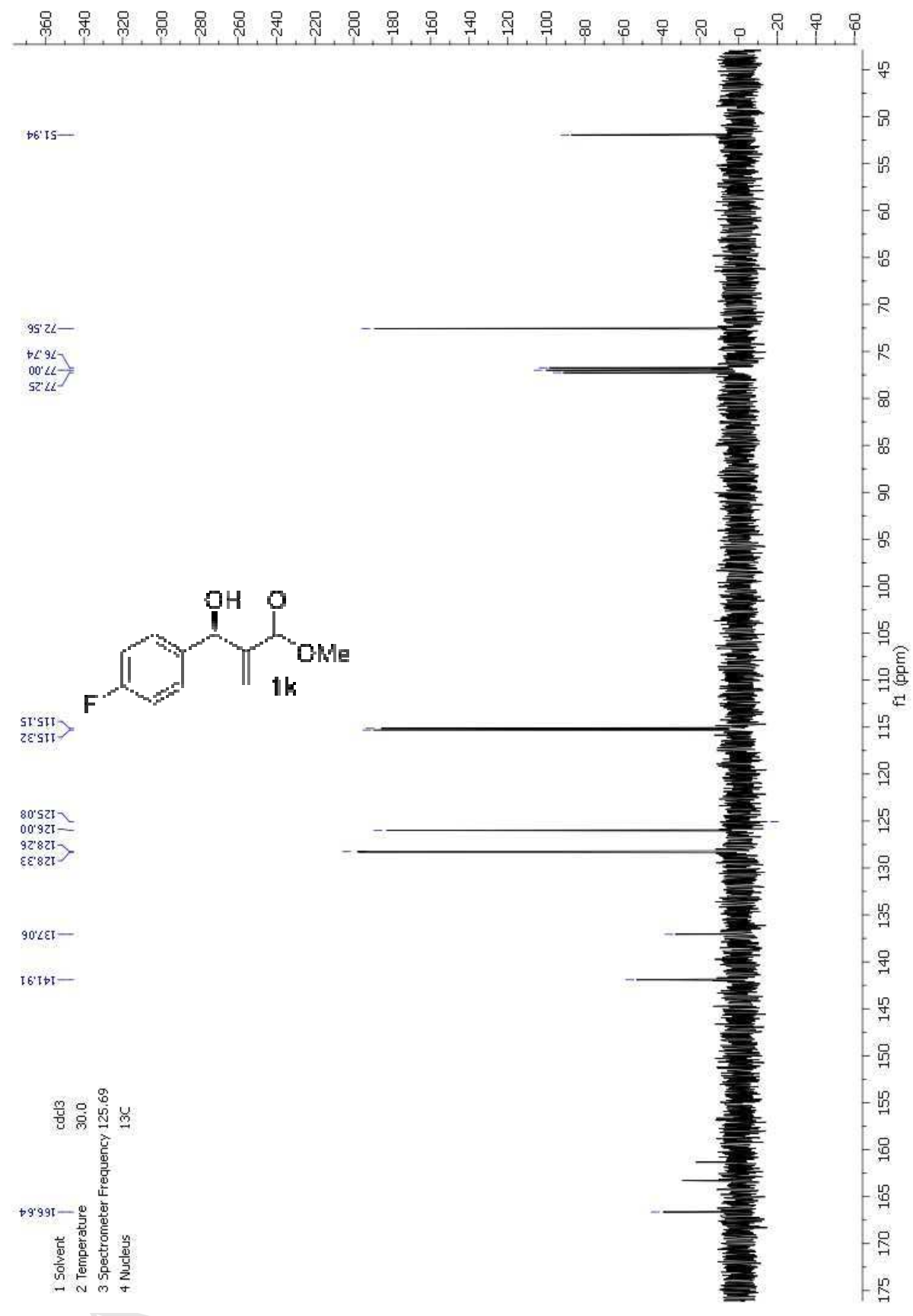




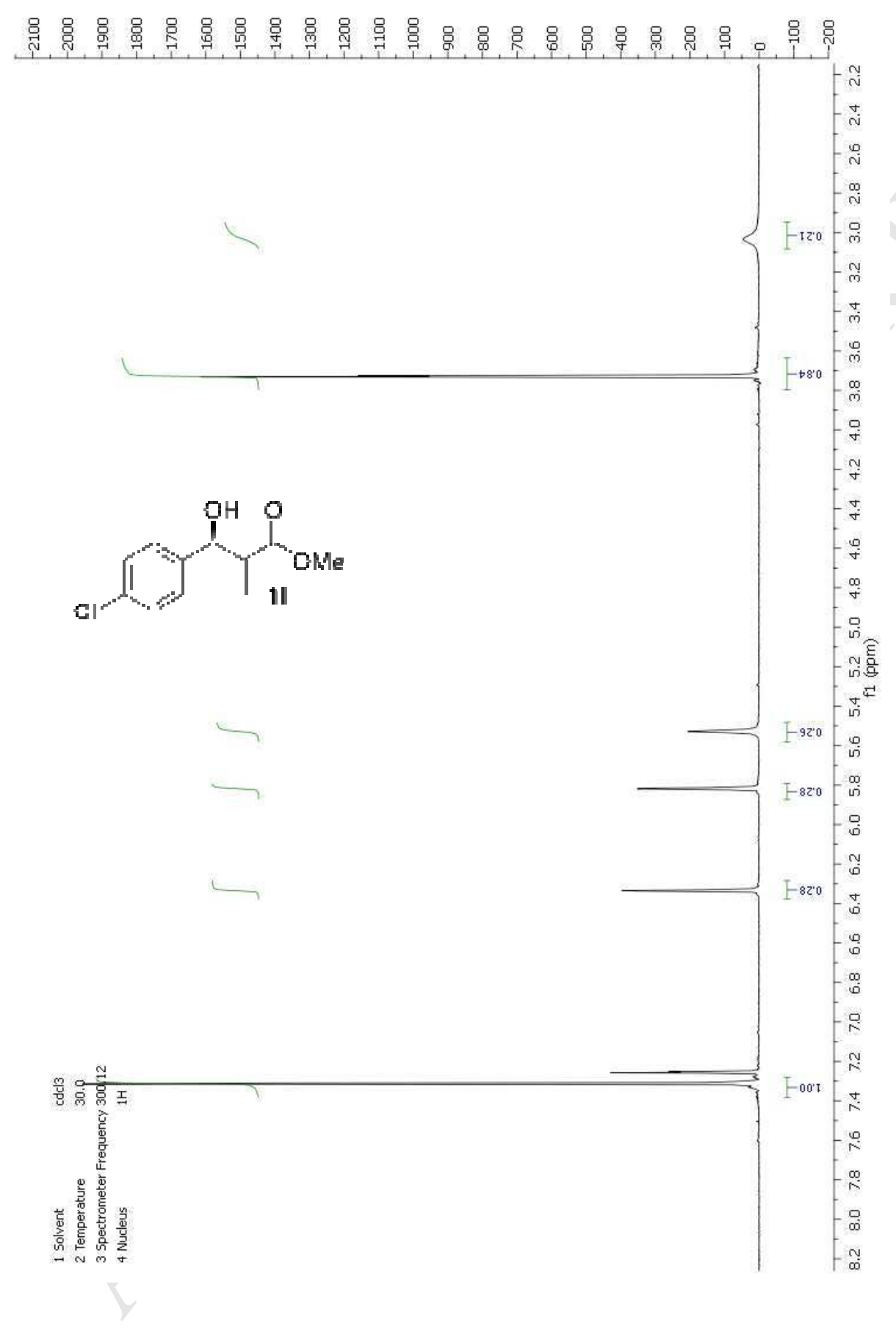




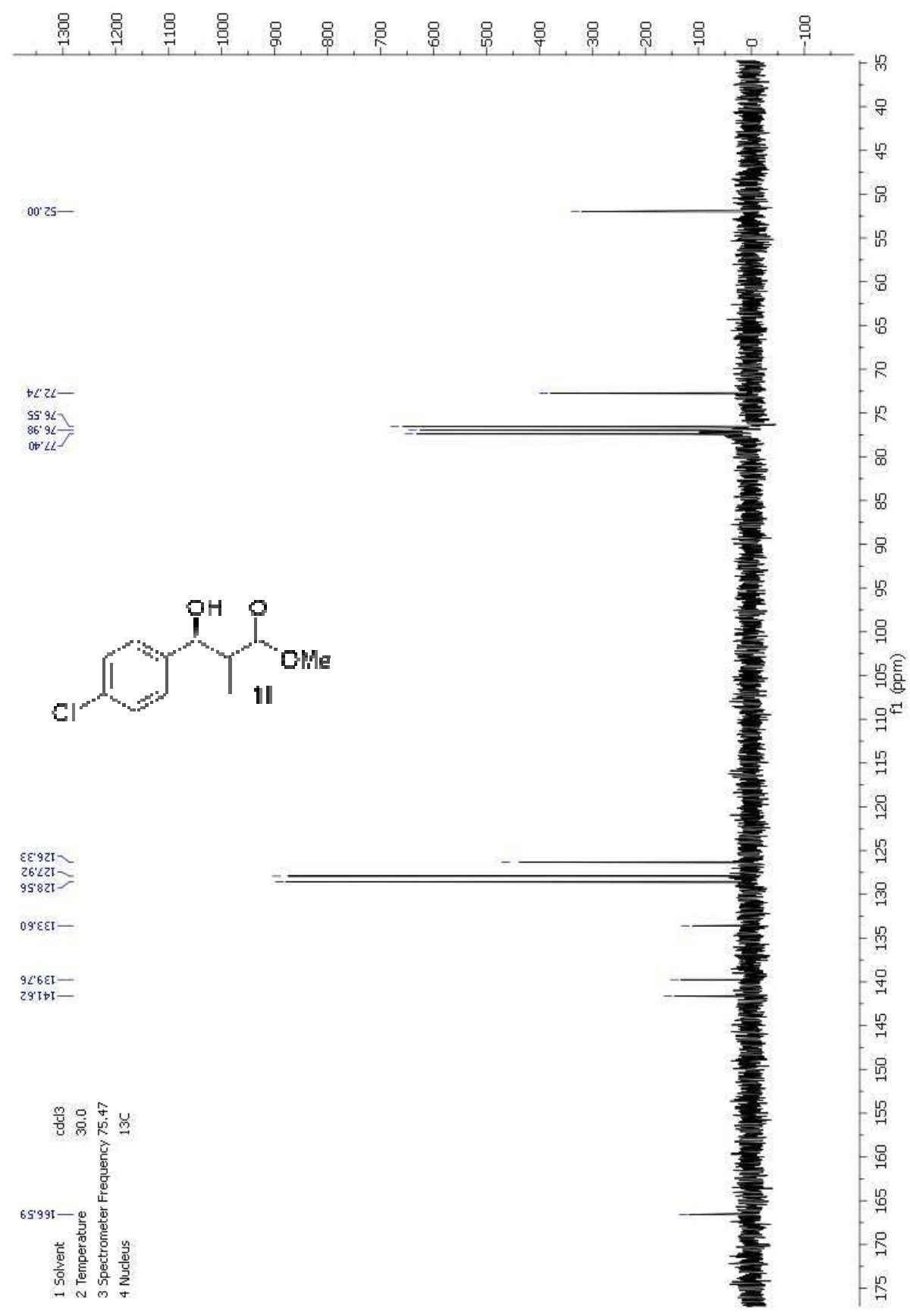




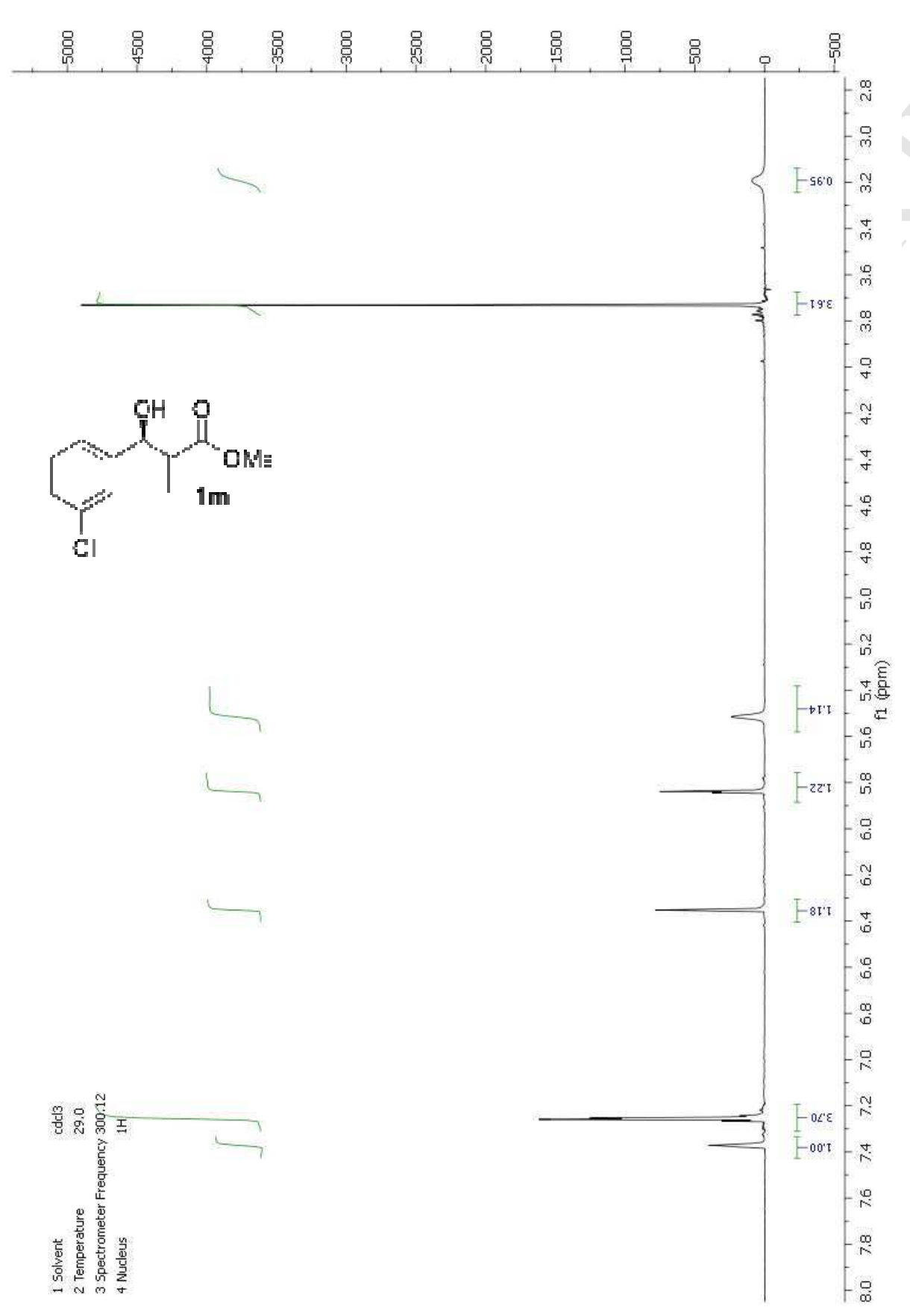




$$
1
$$




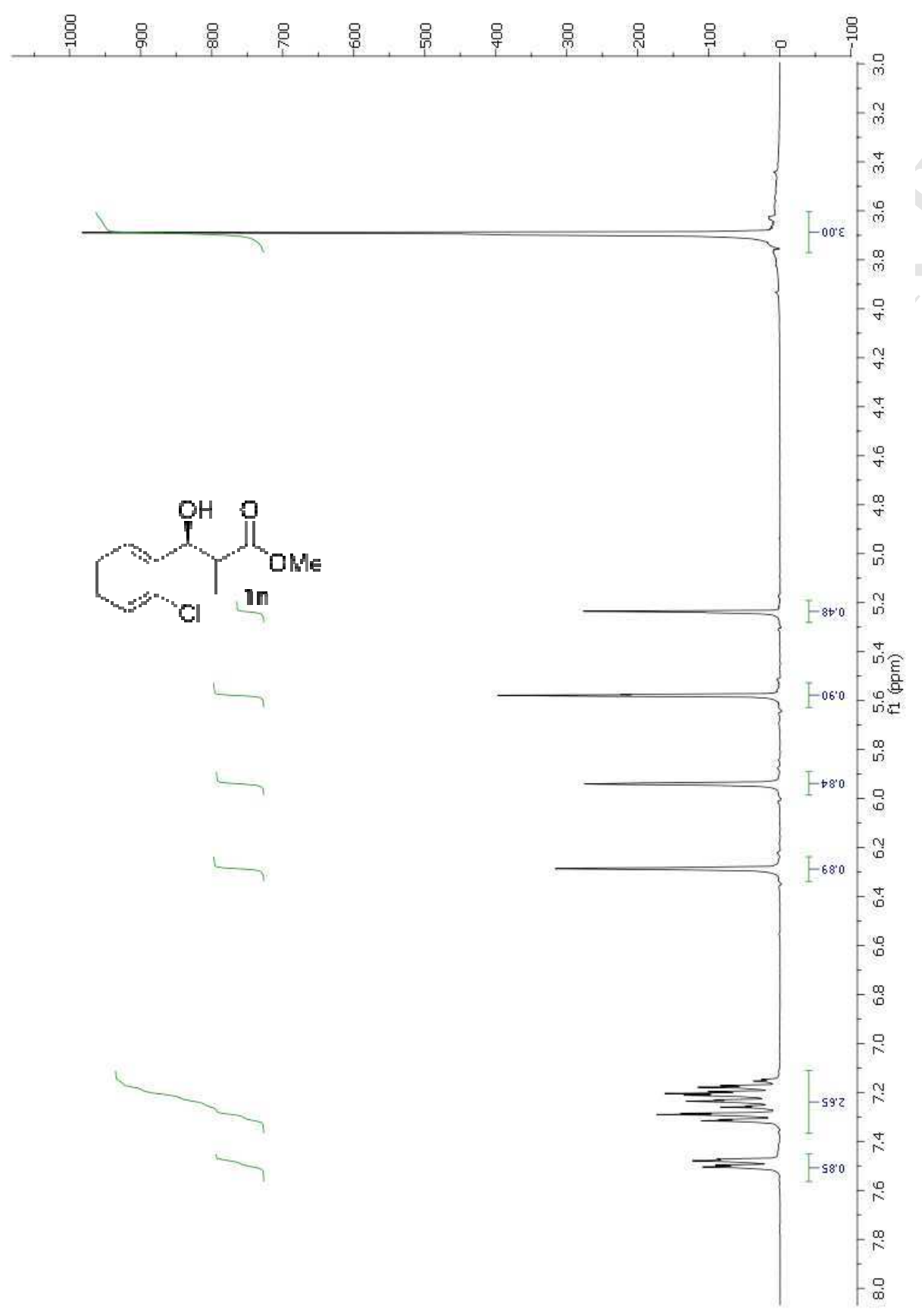




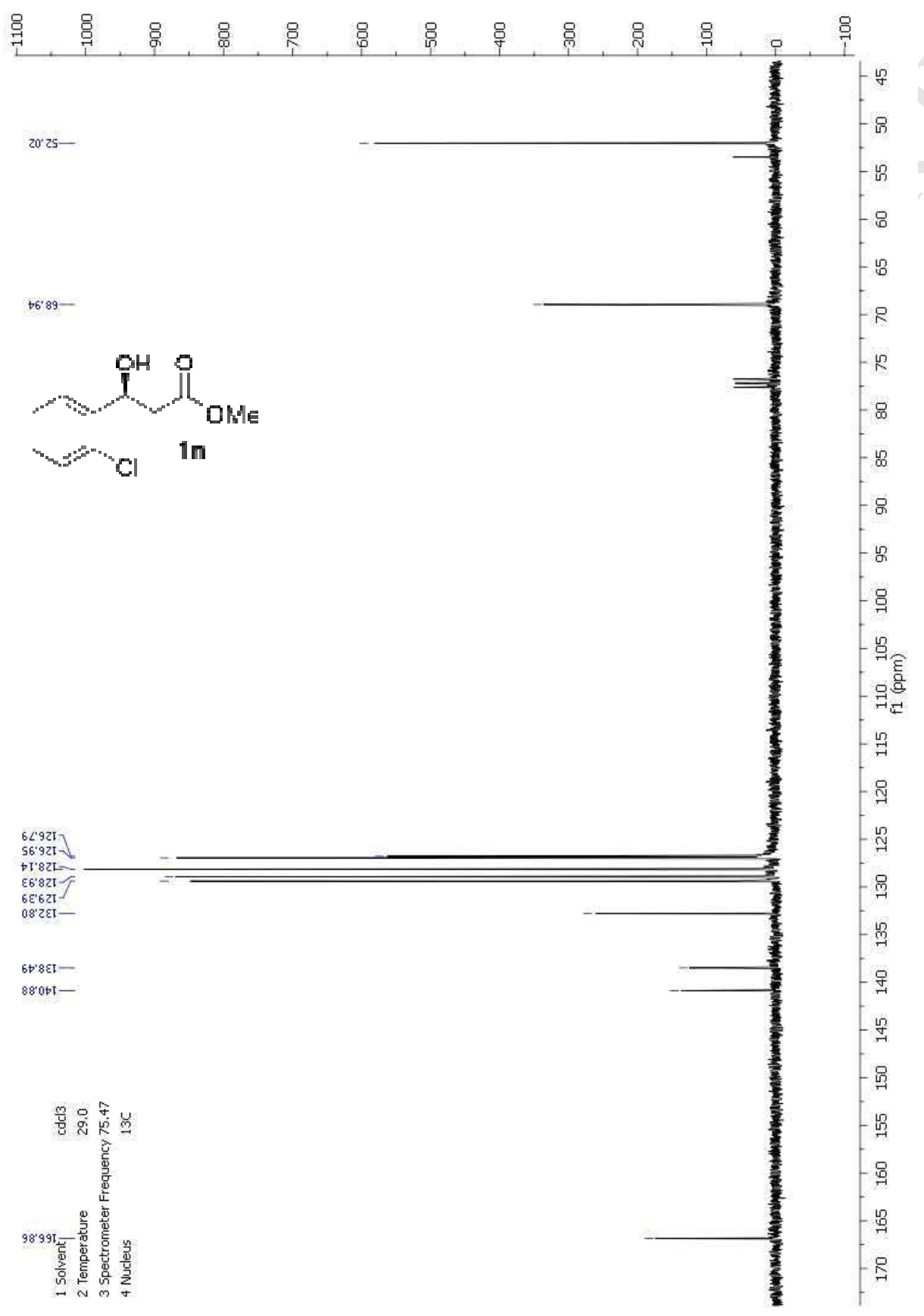




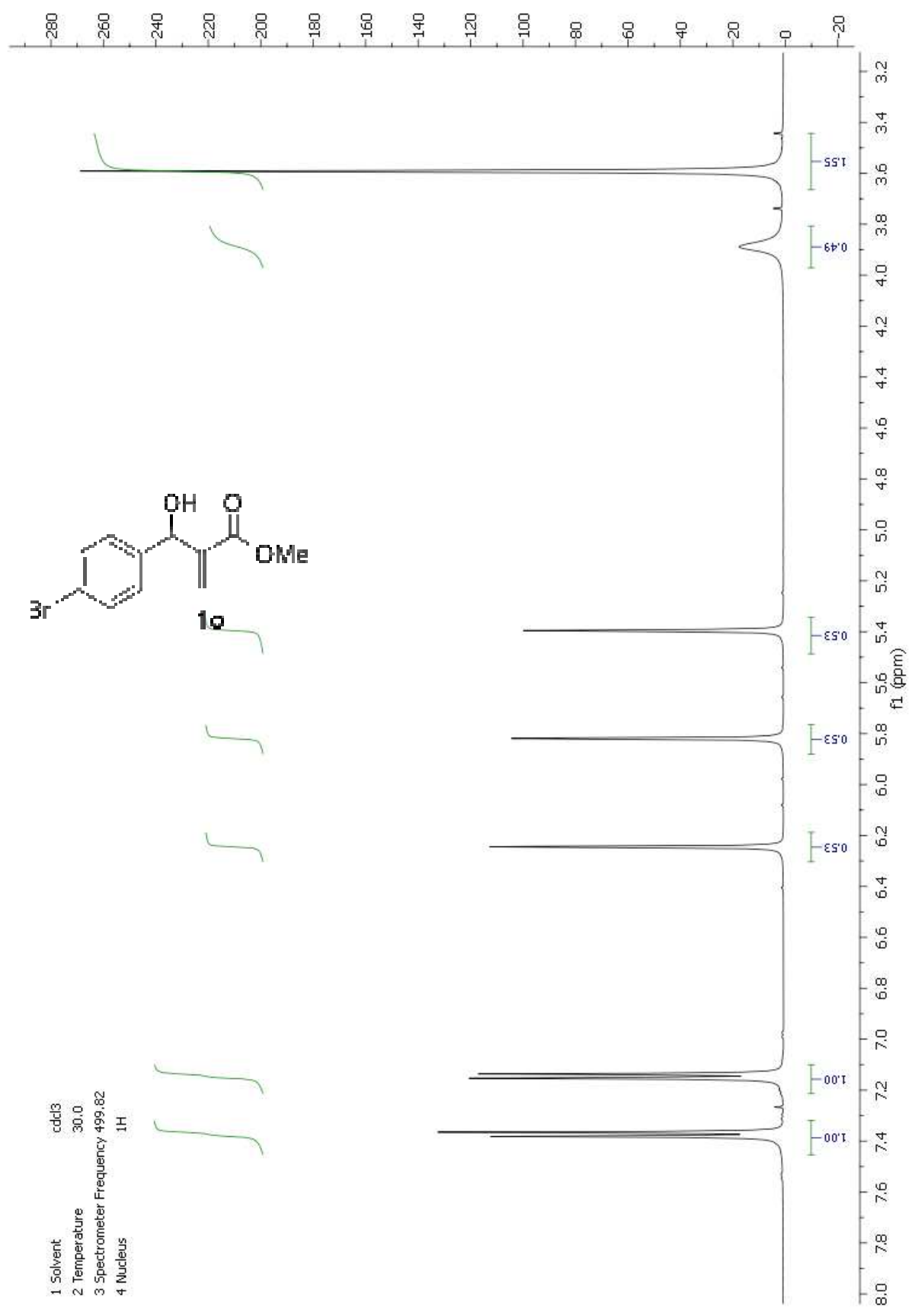




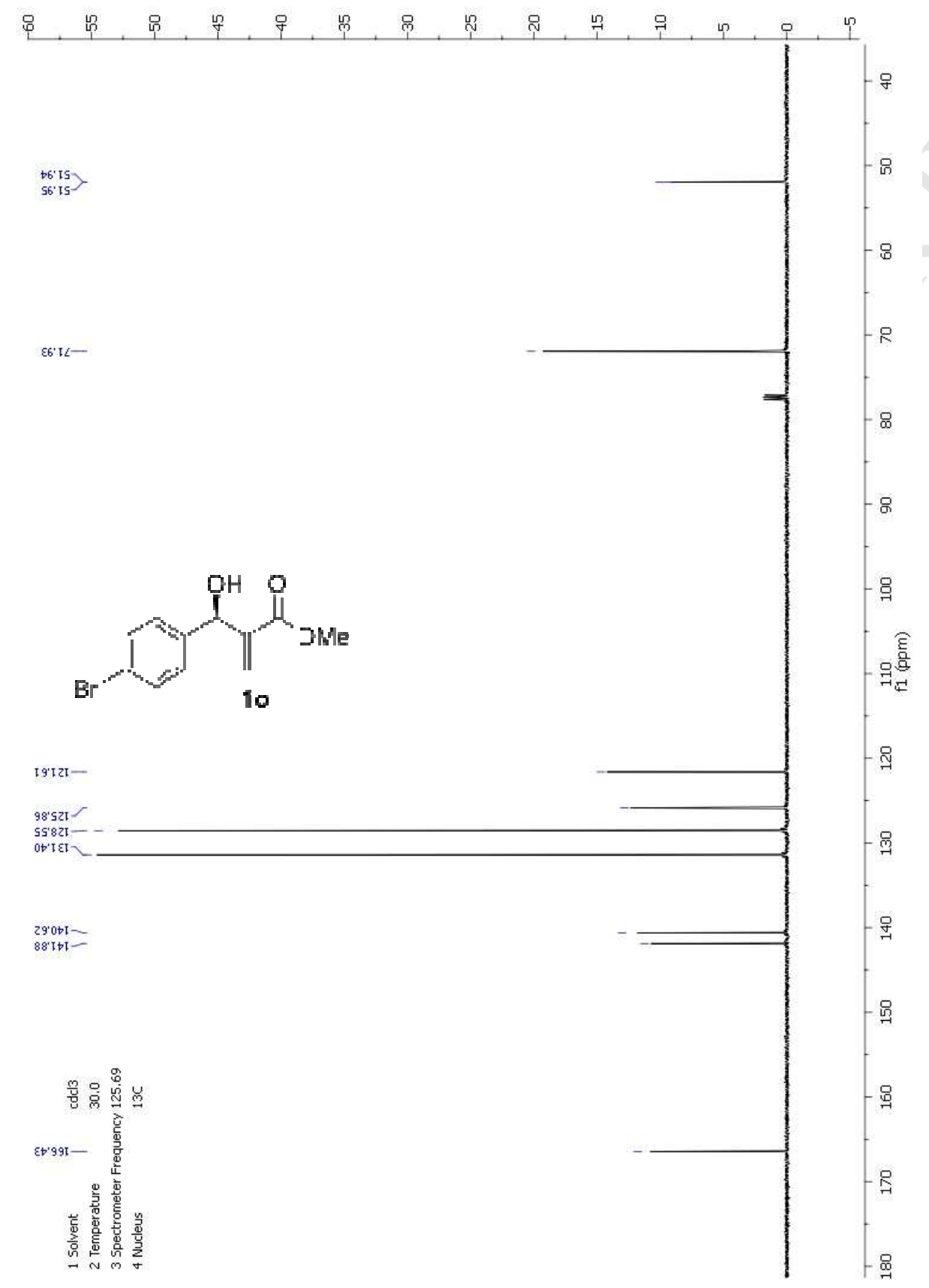




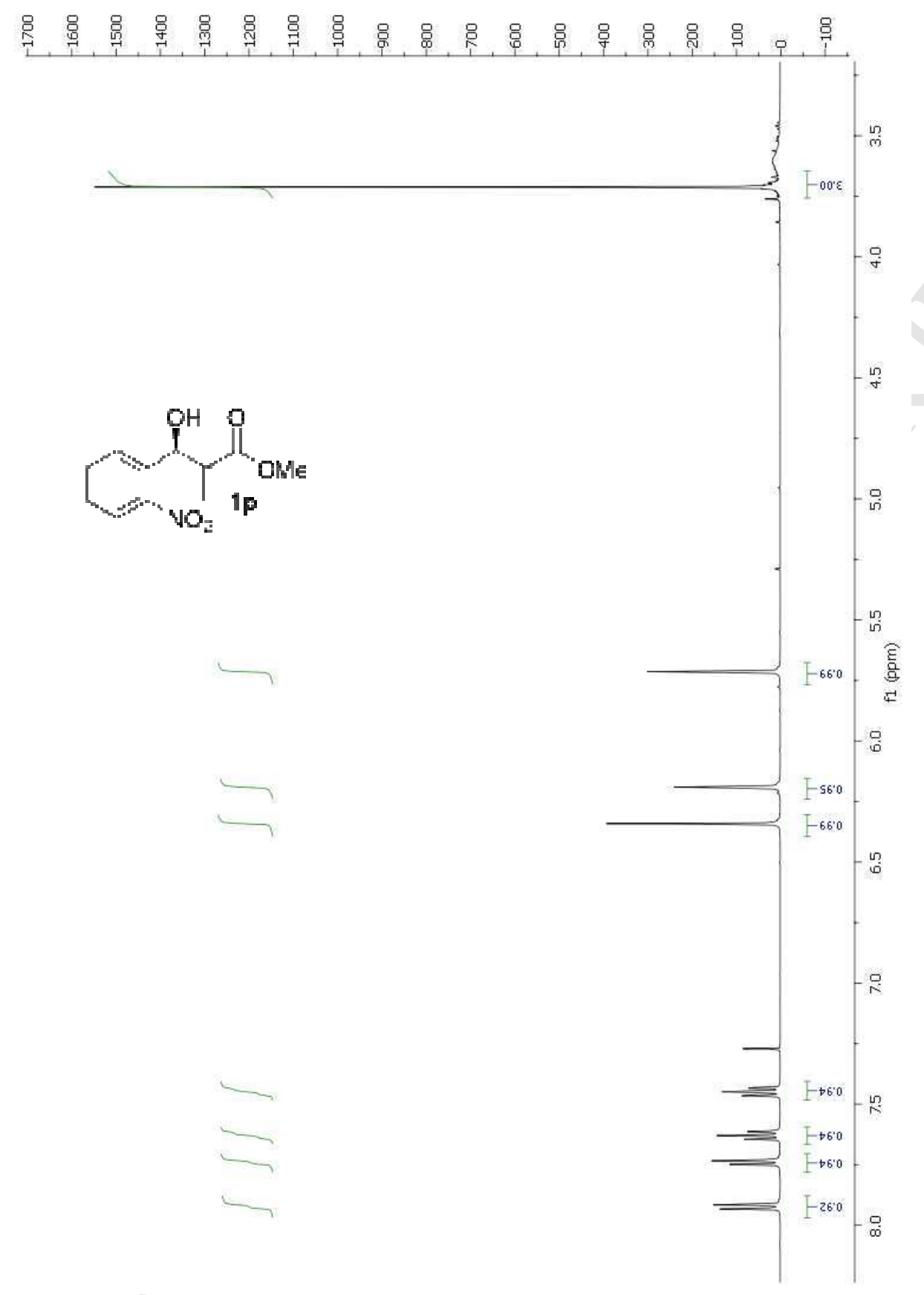




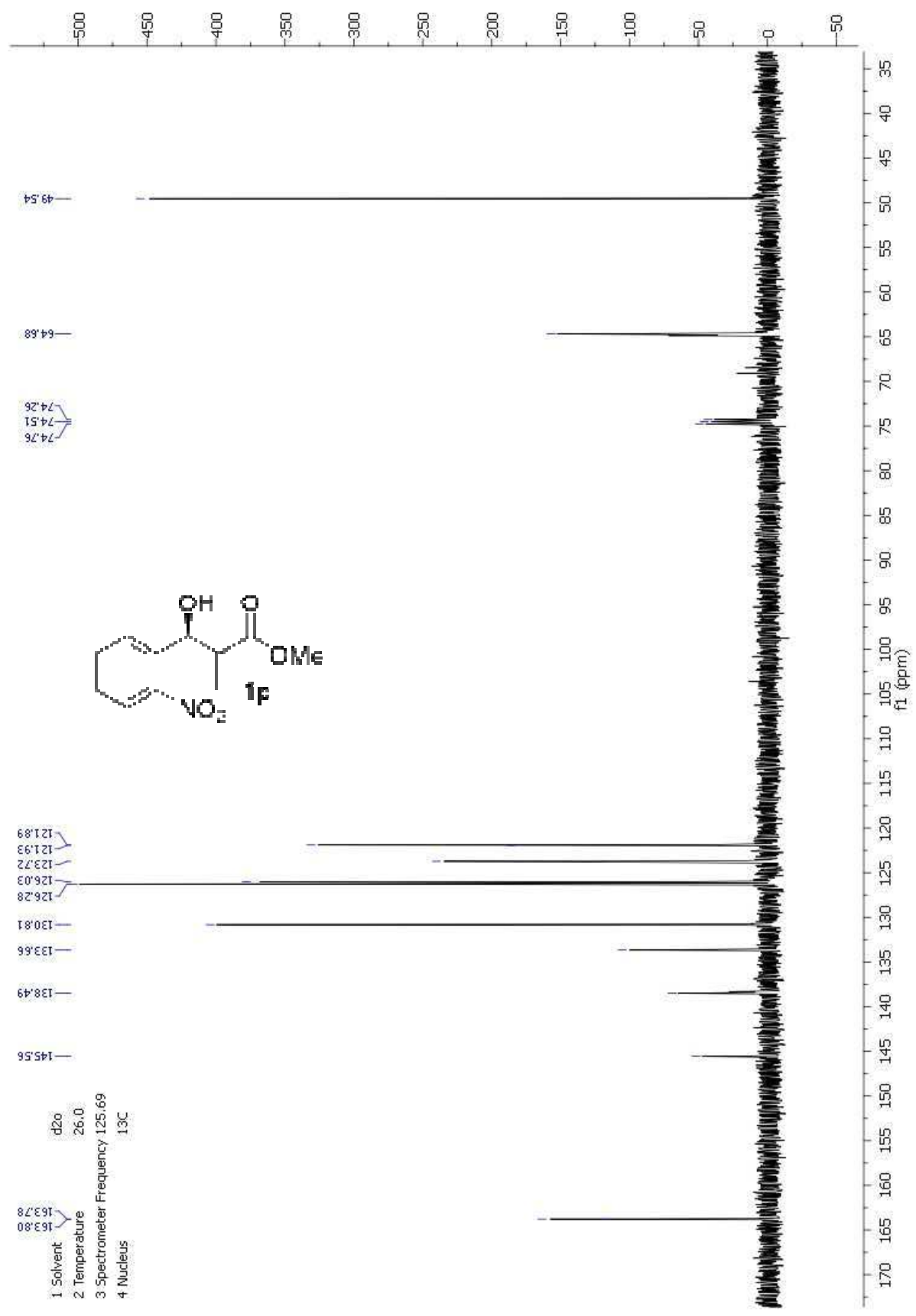




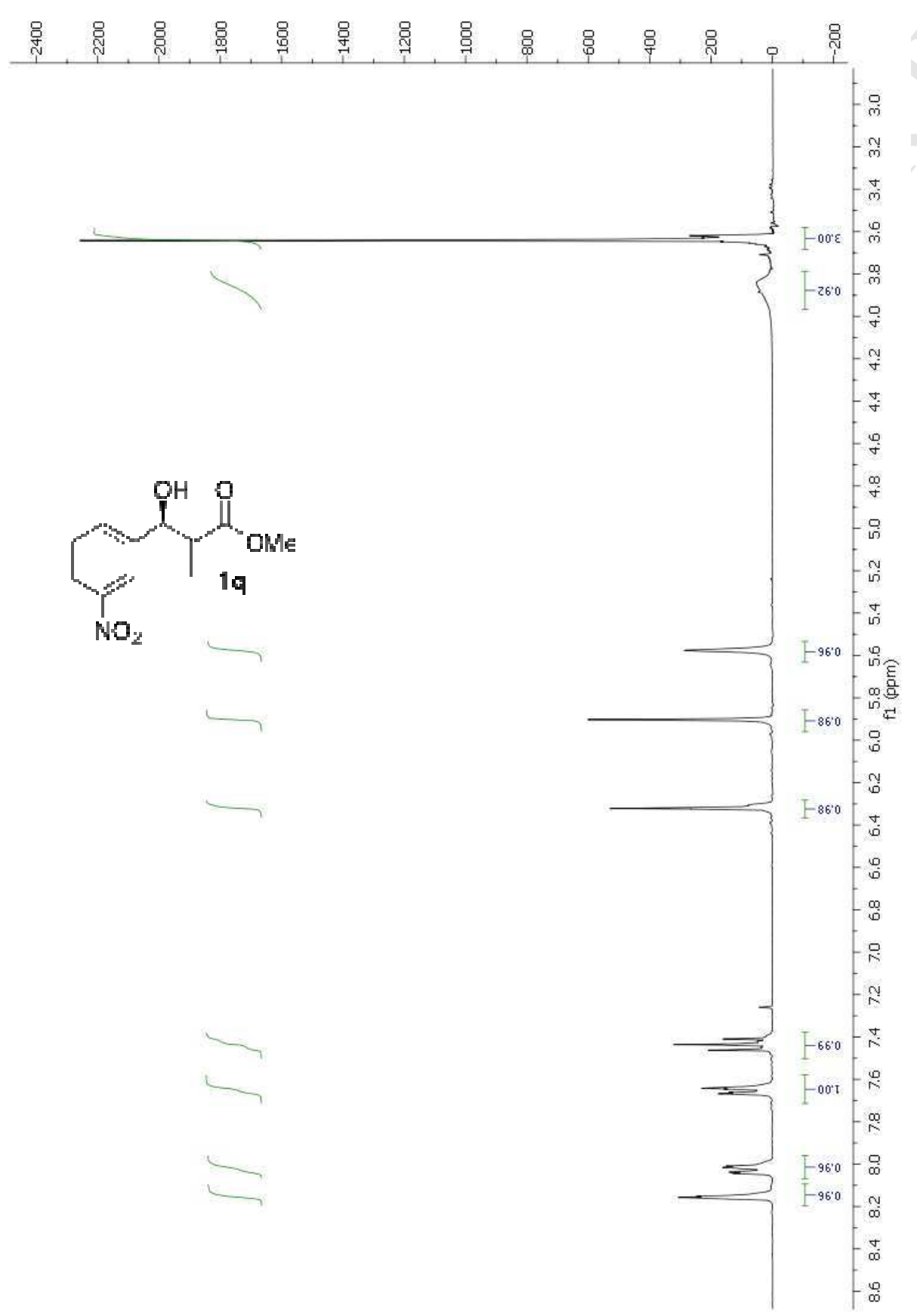




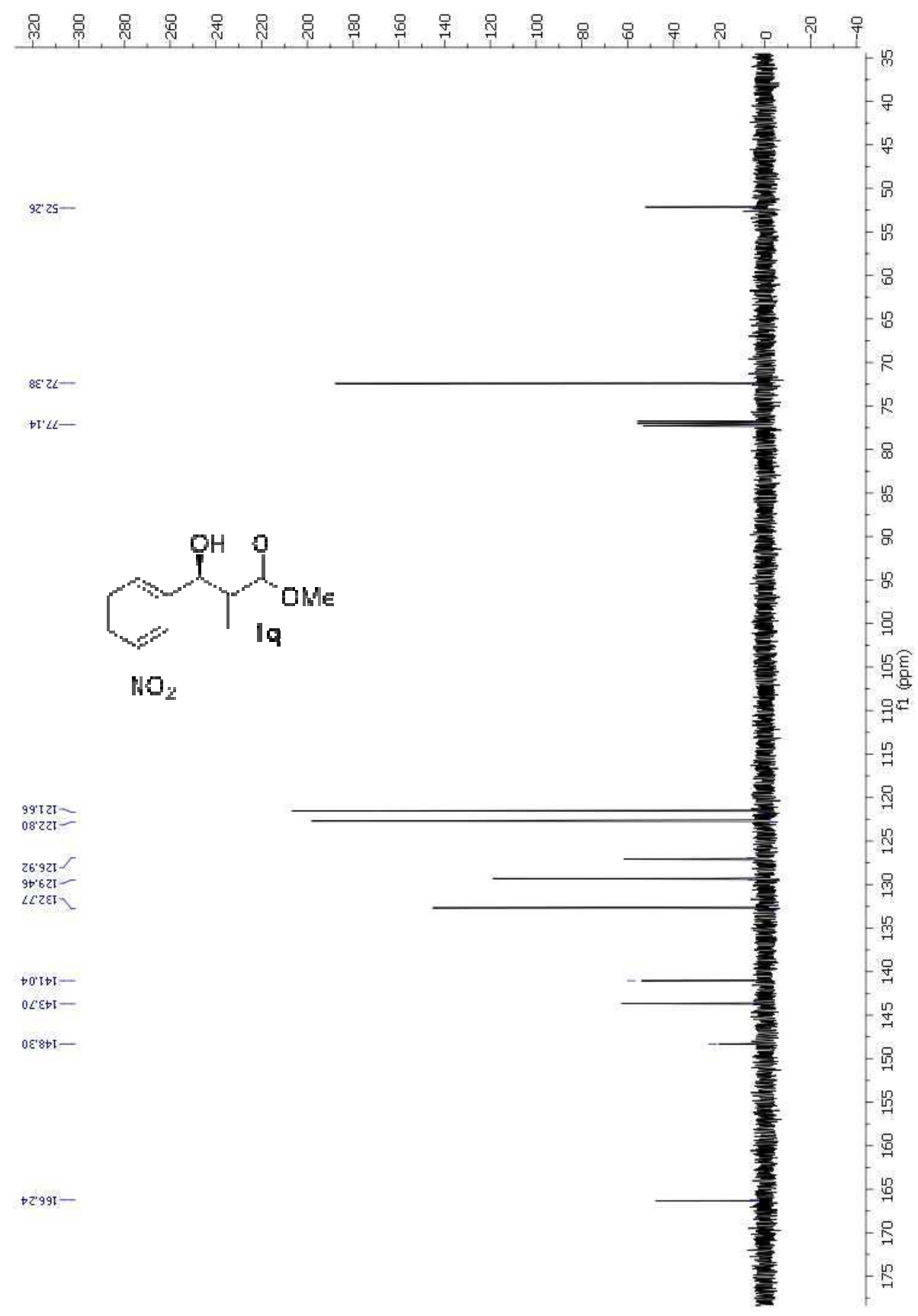




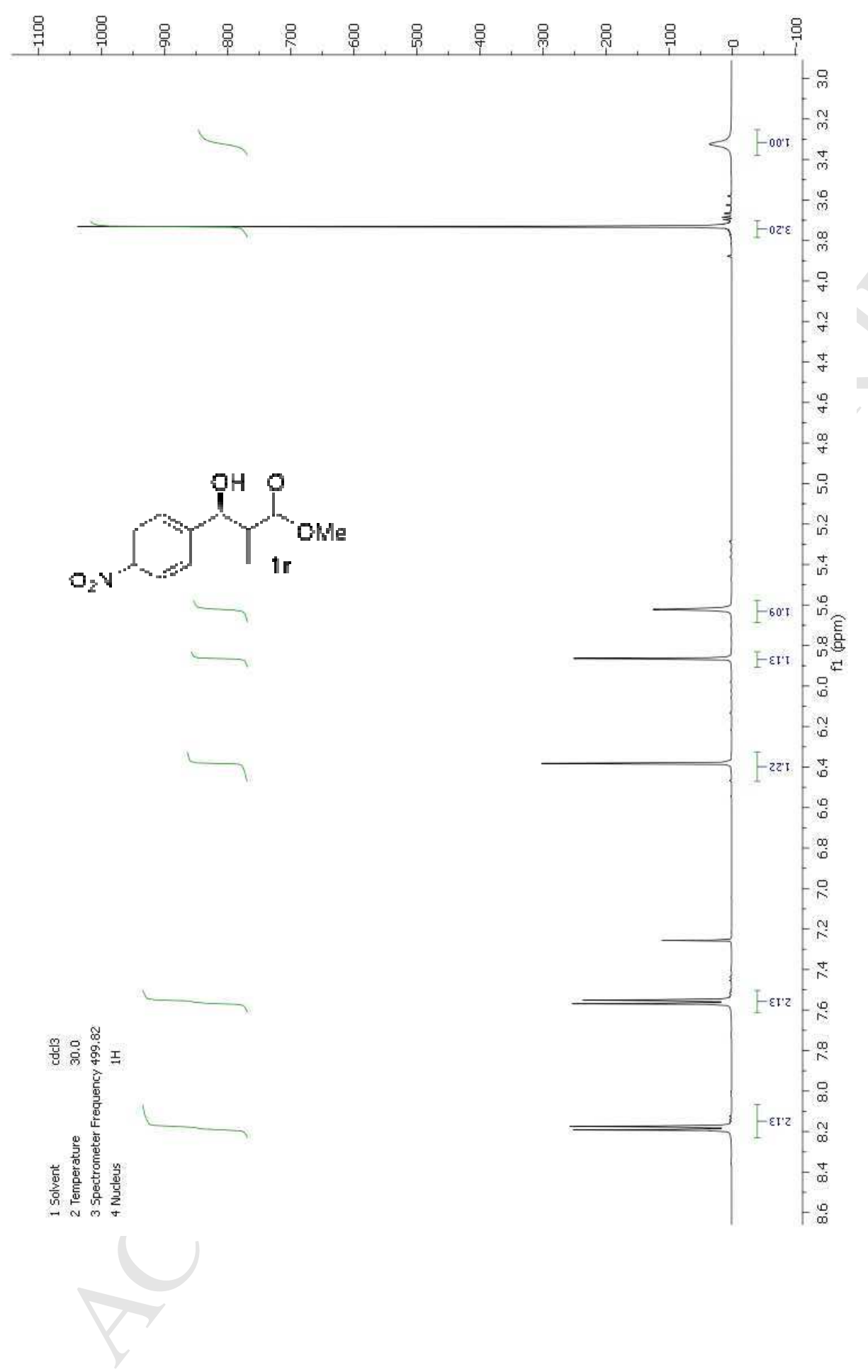




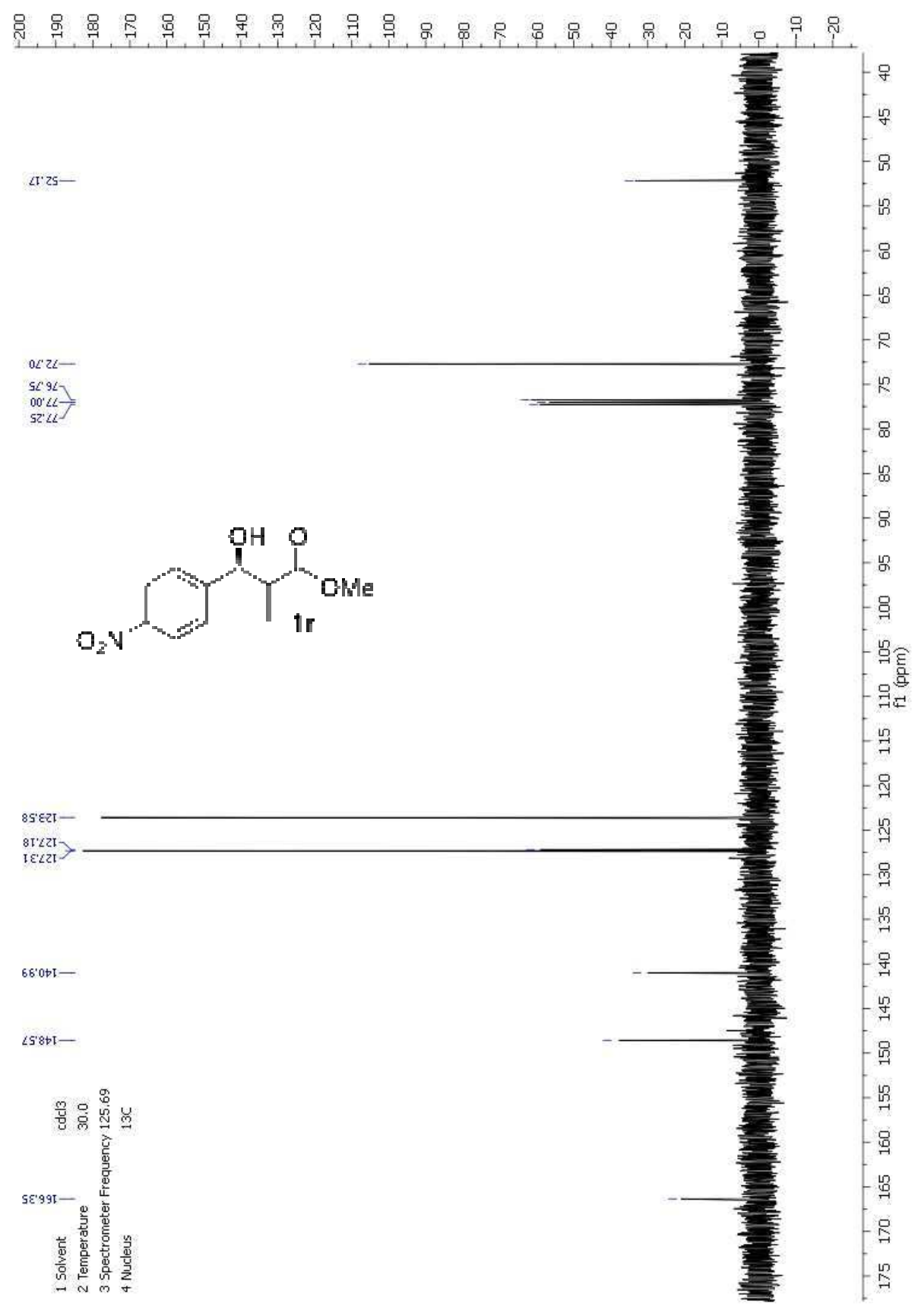




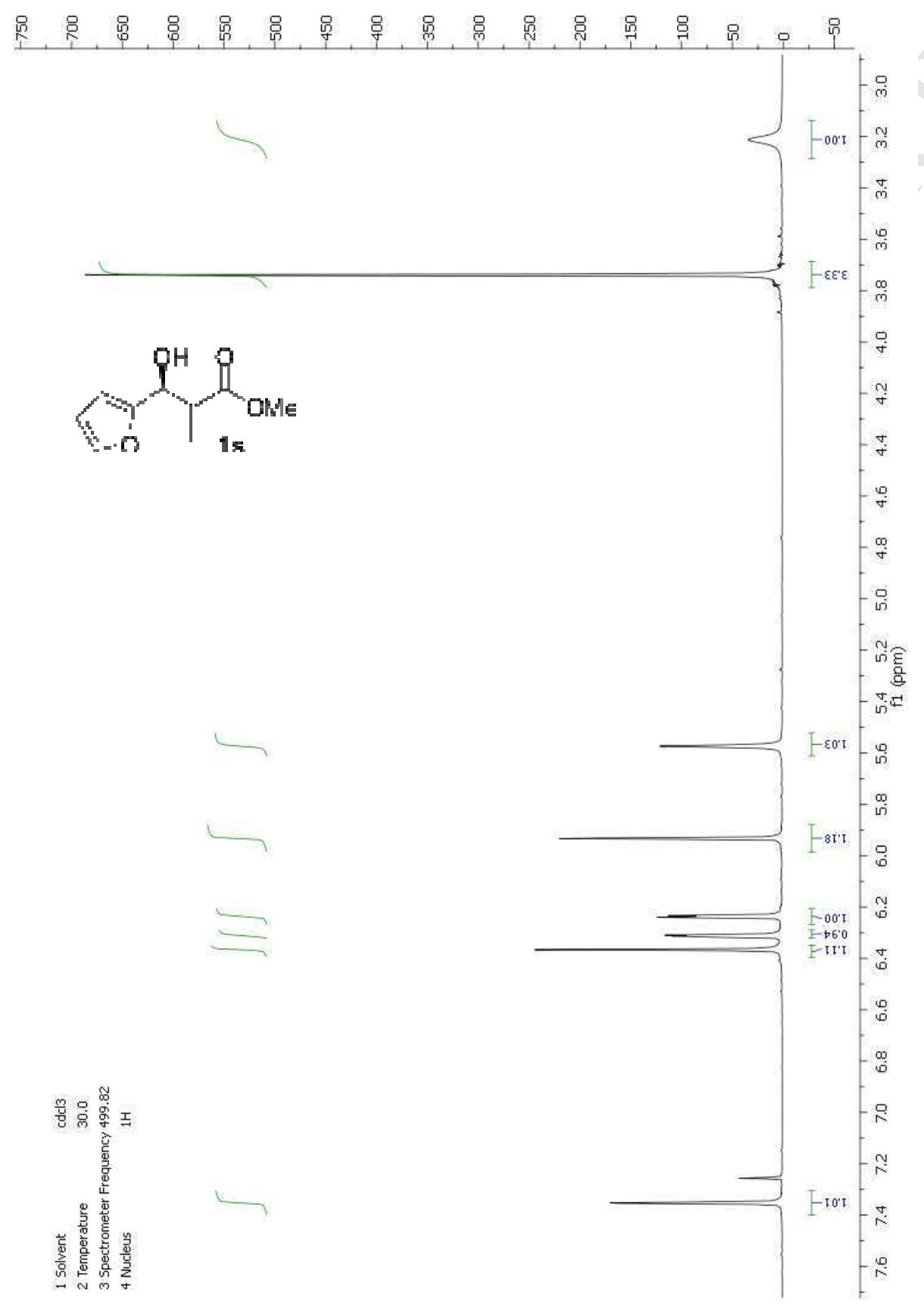




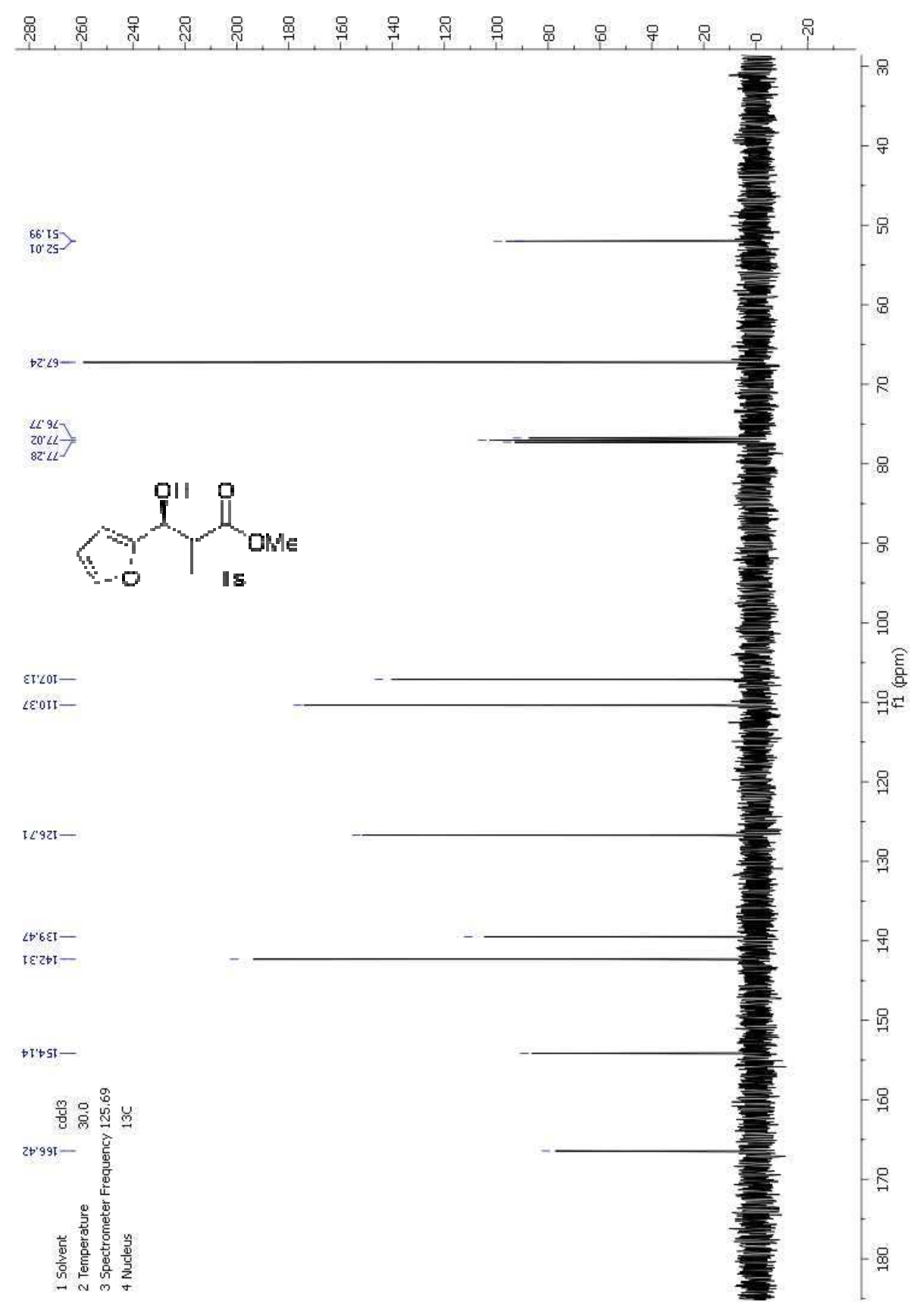




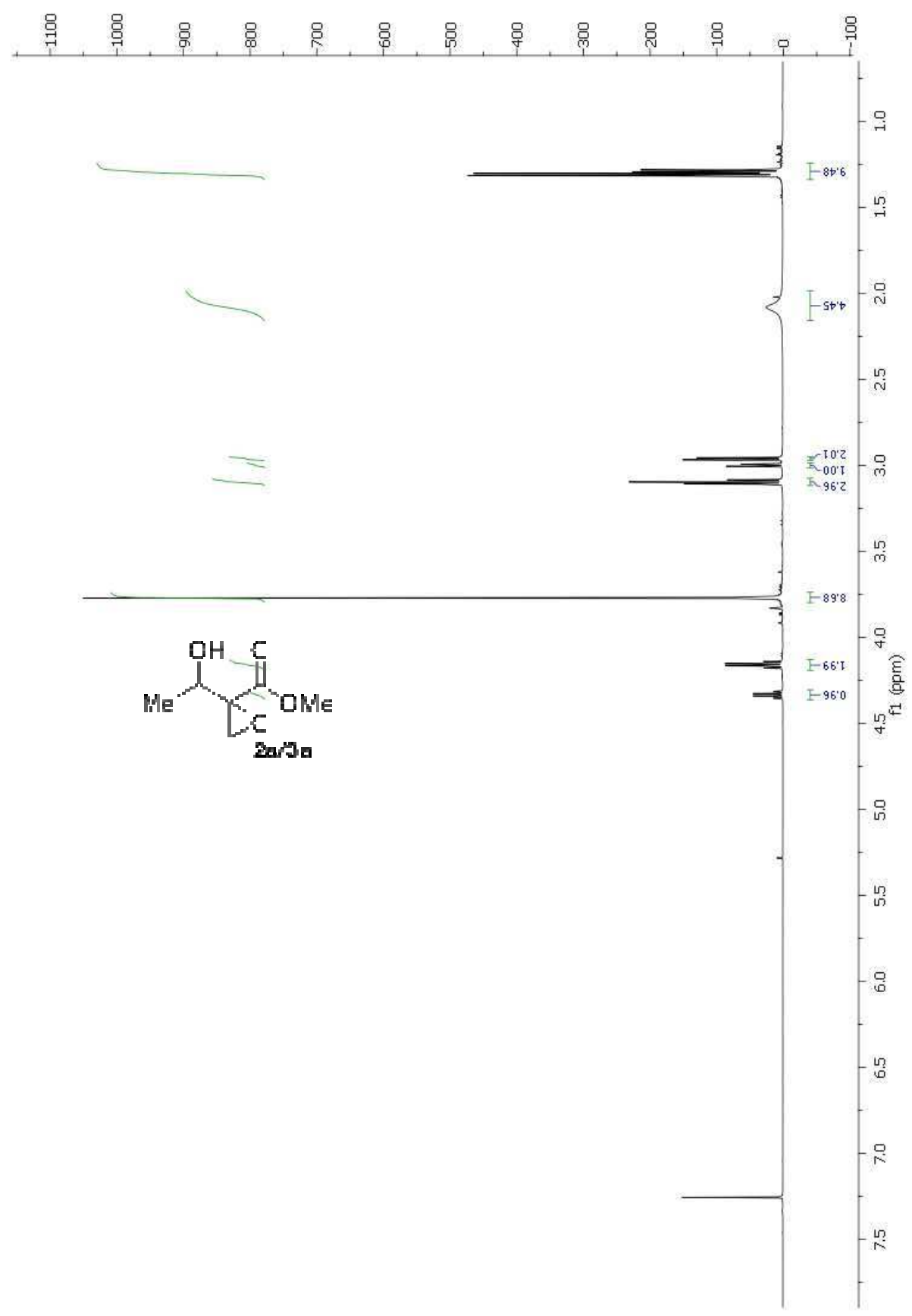




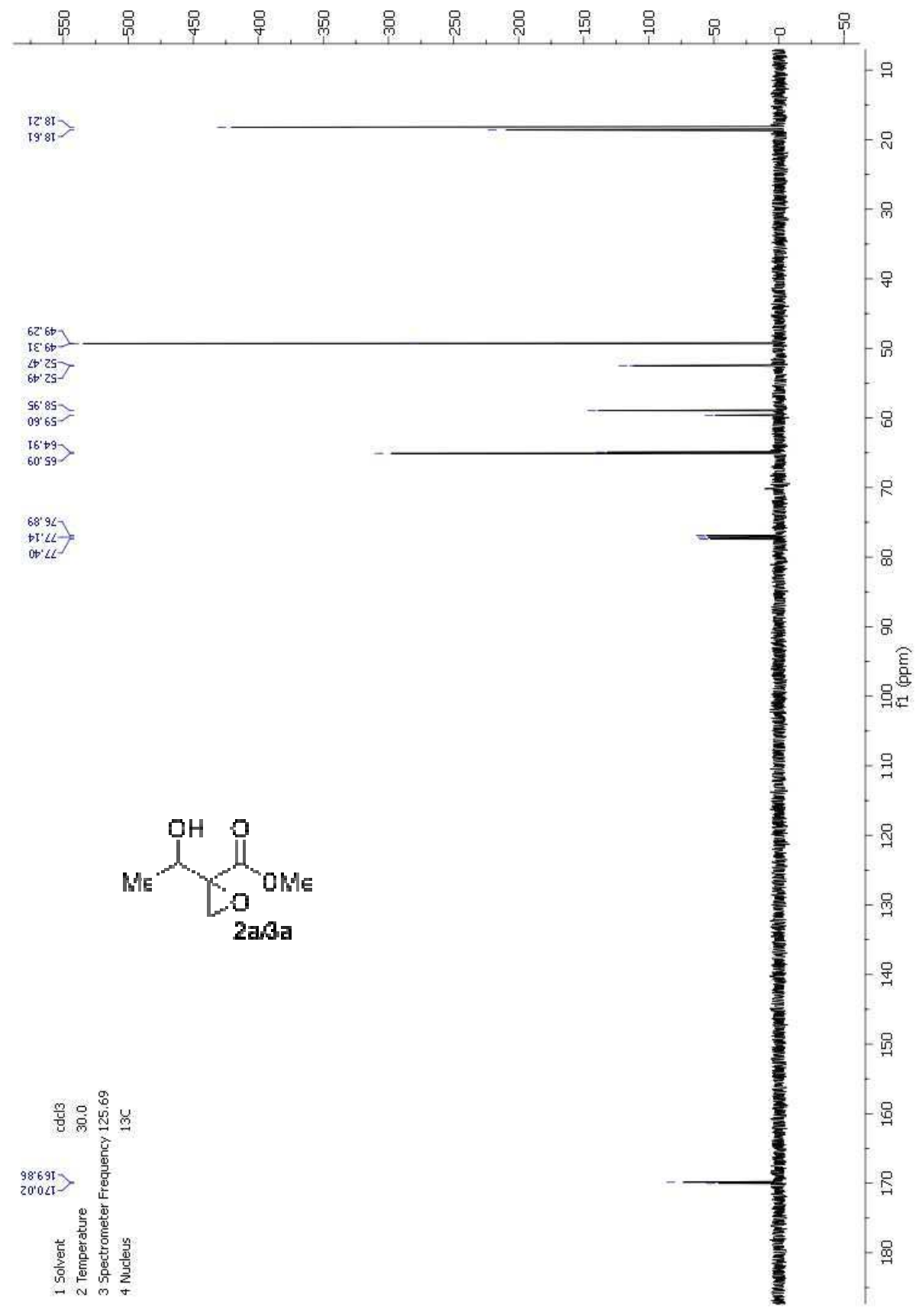




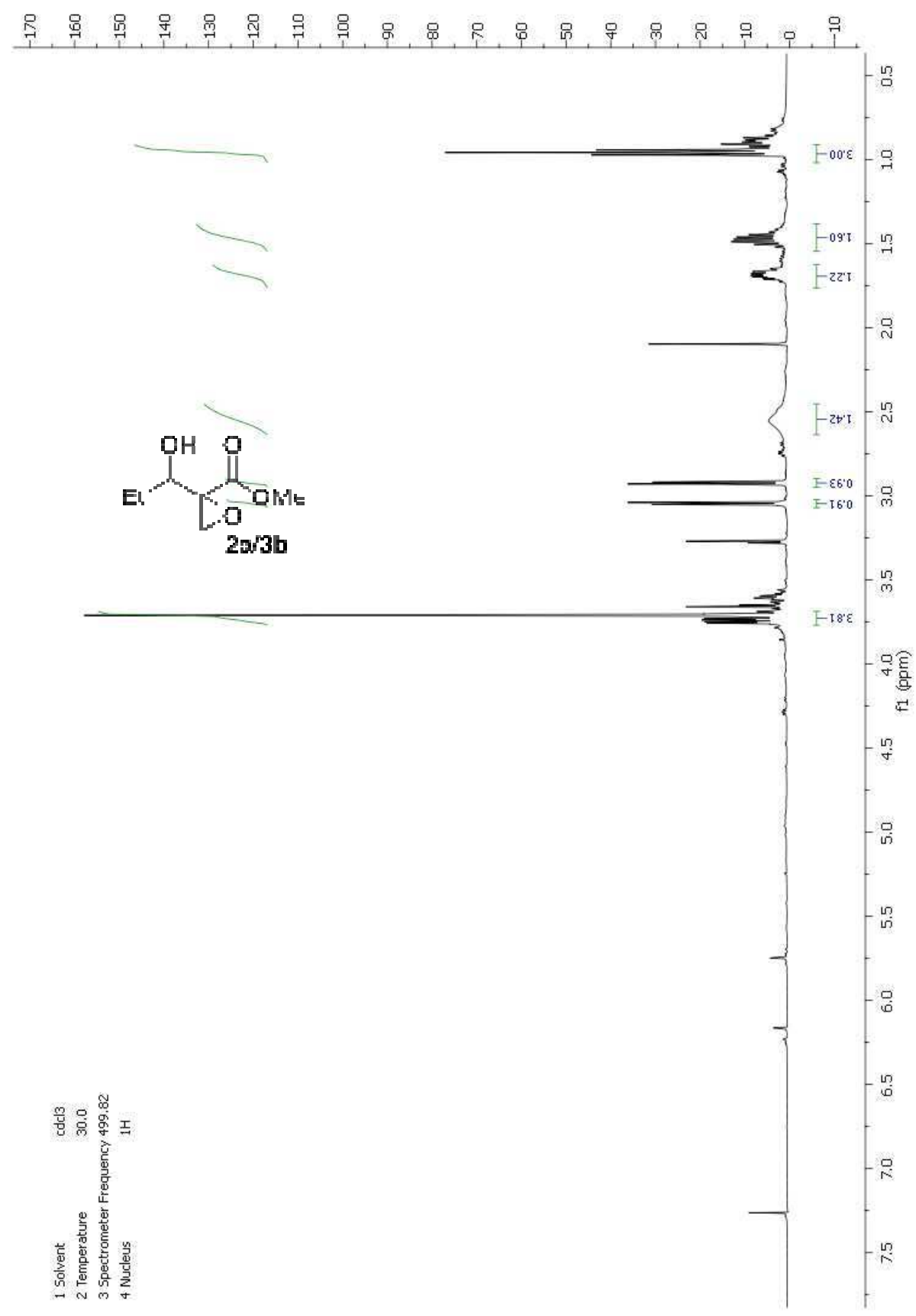




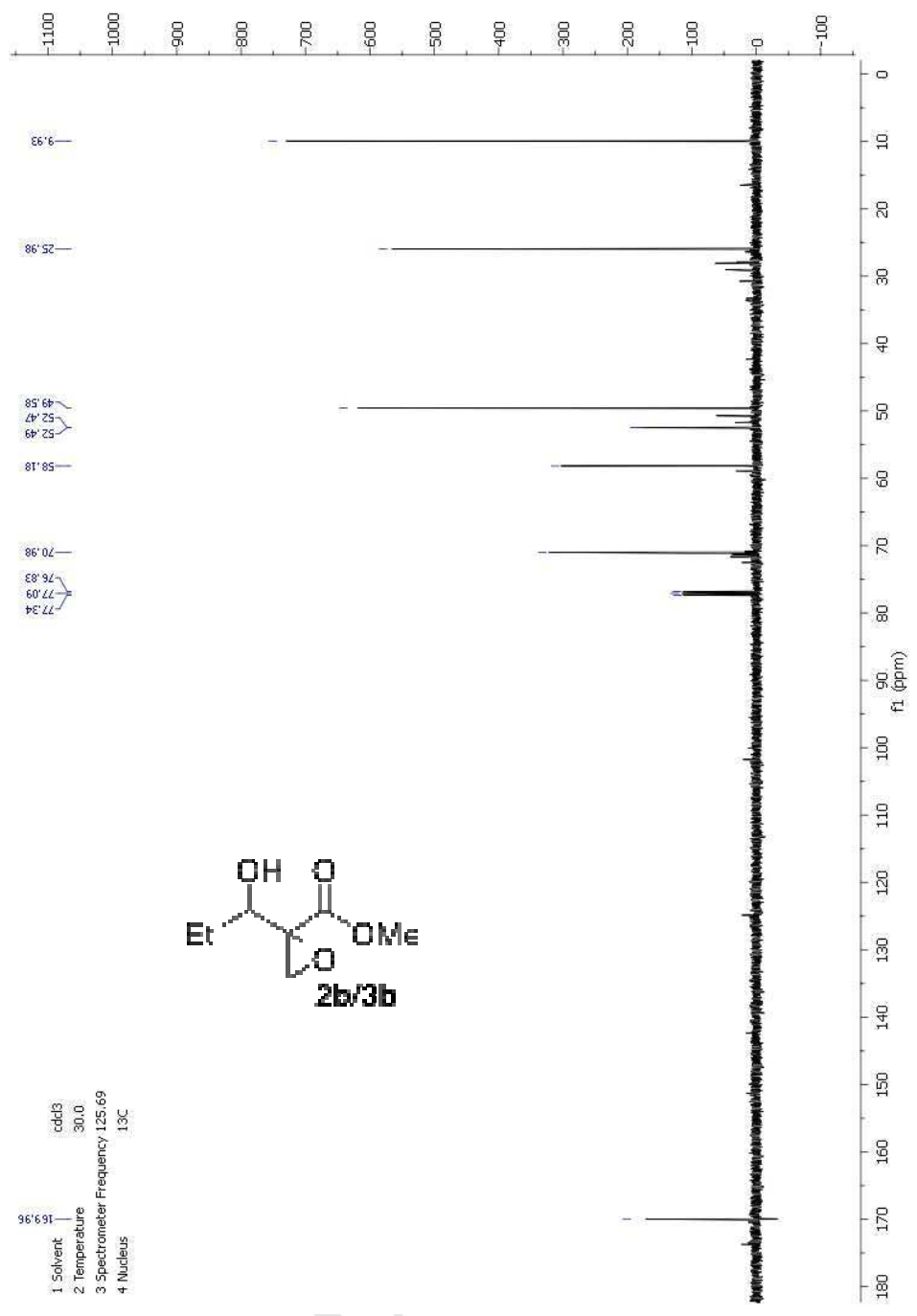




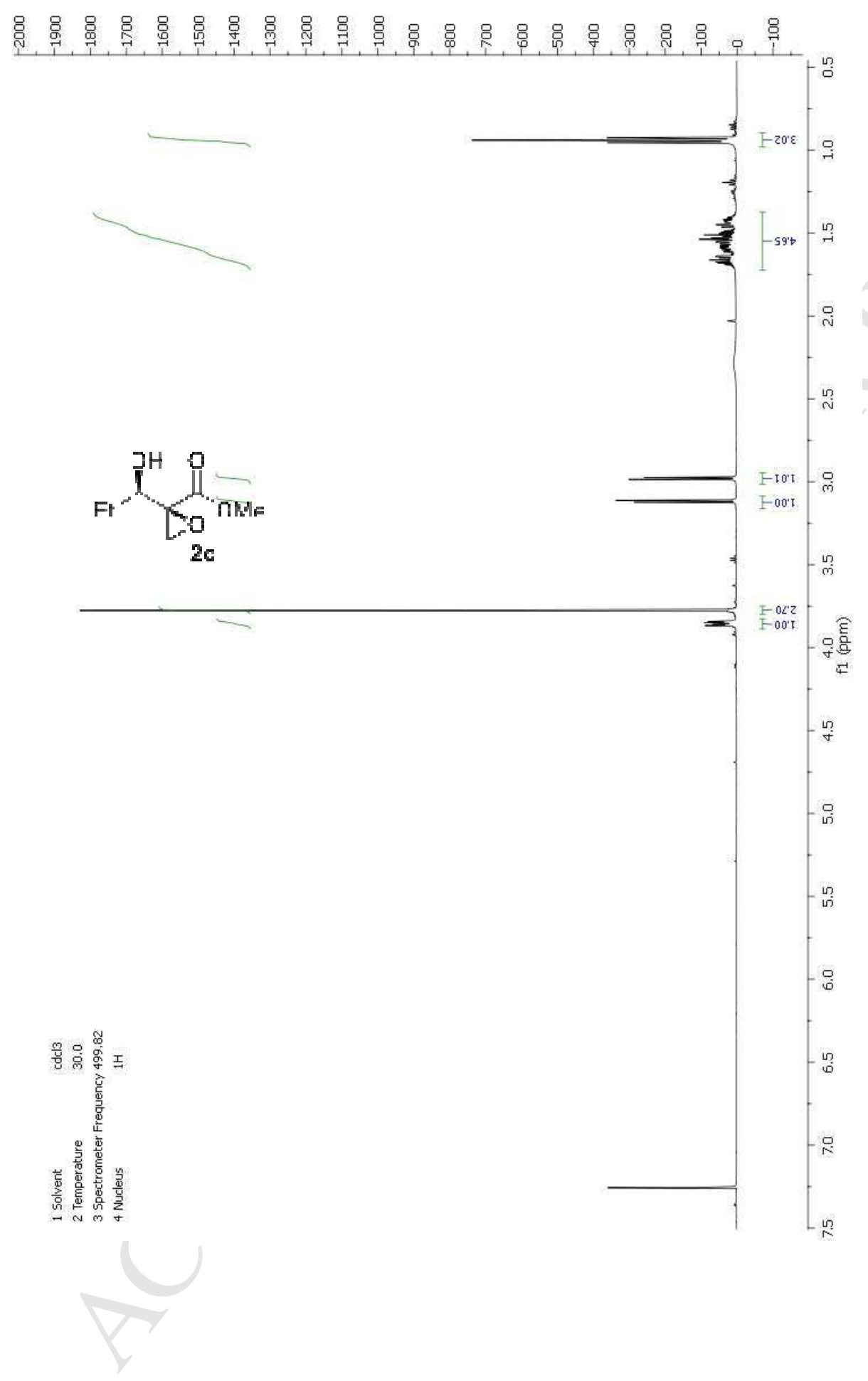




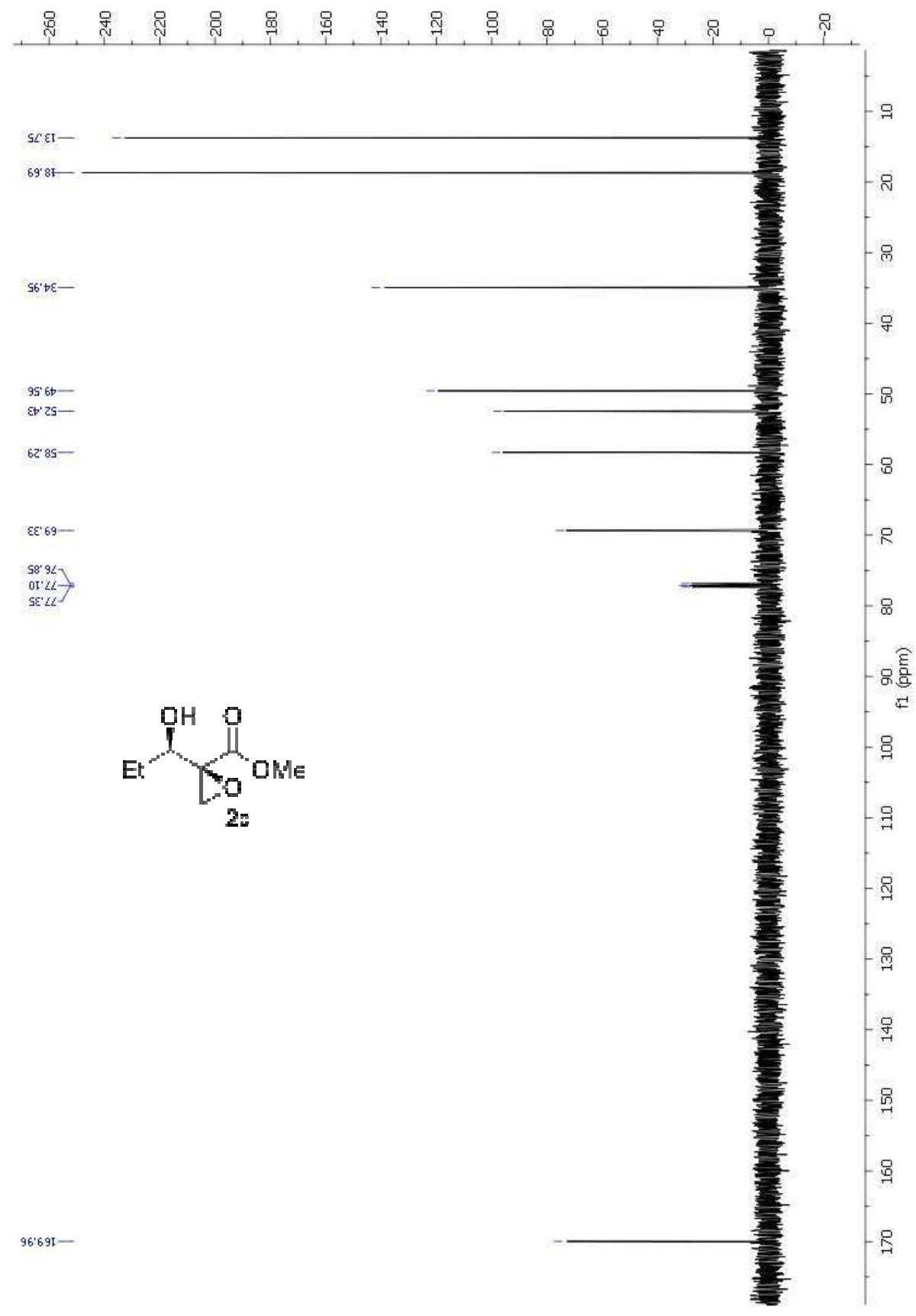




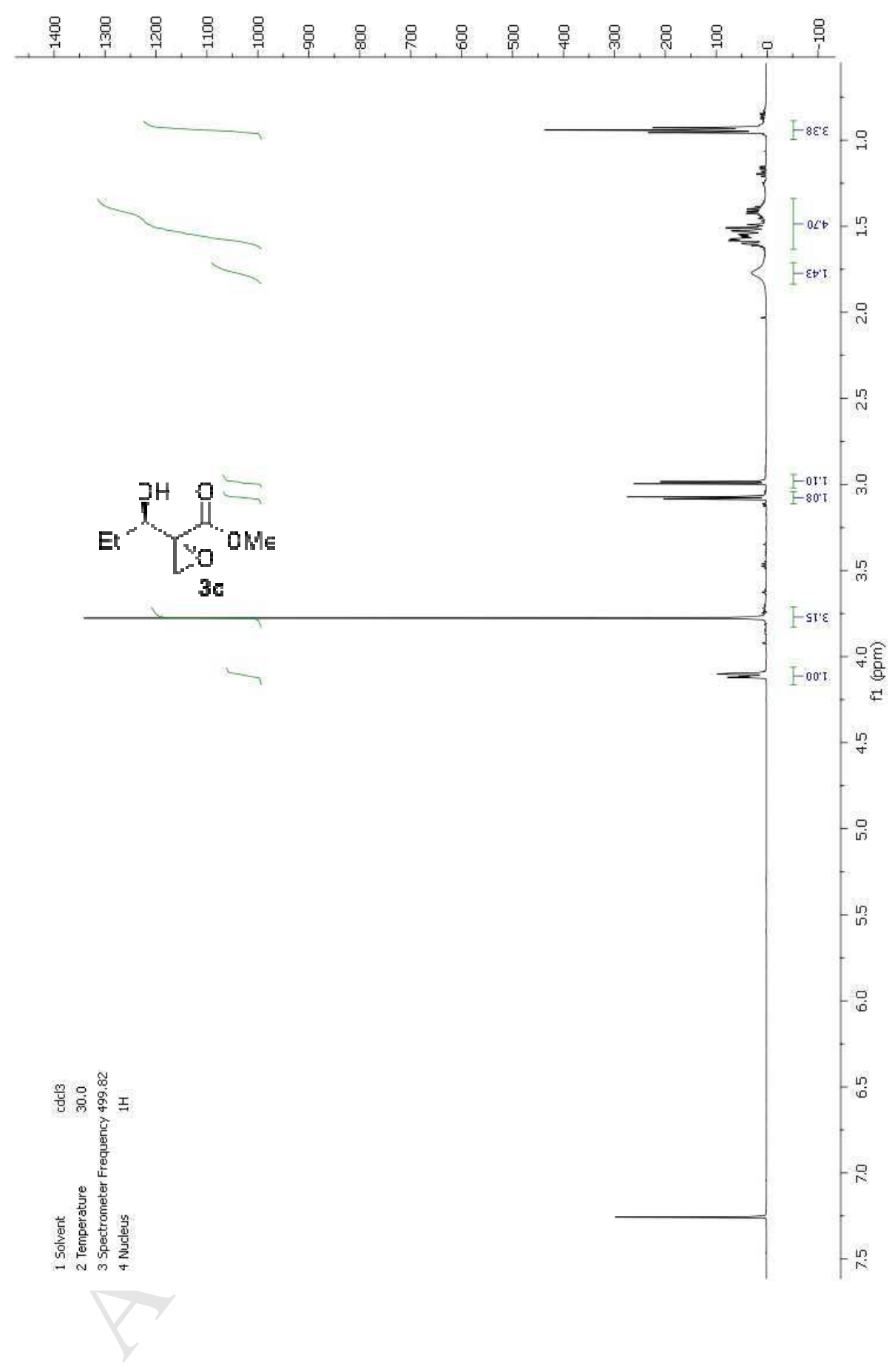




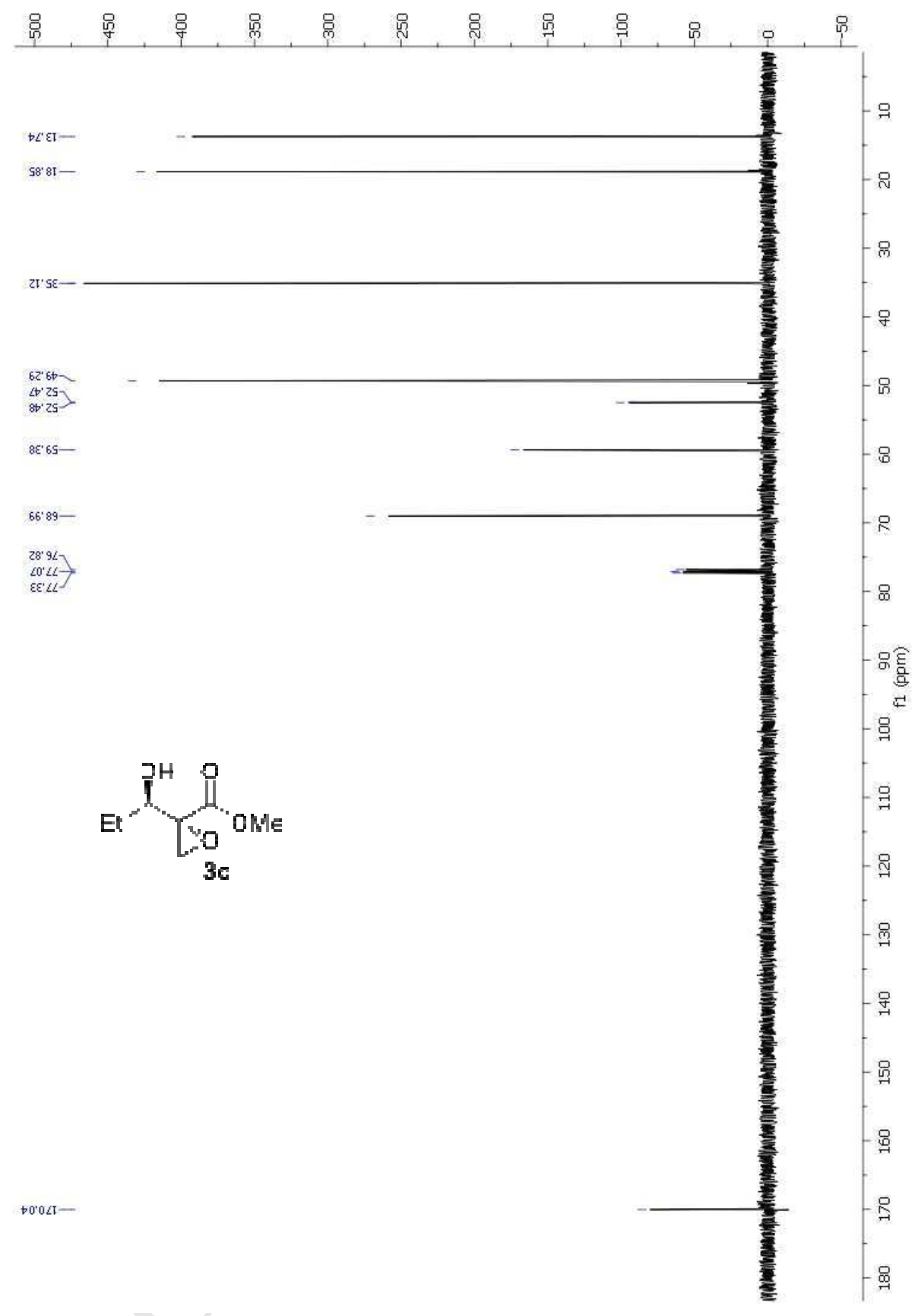




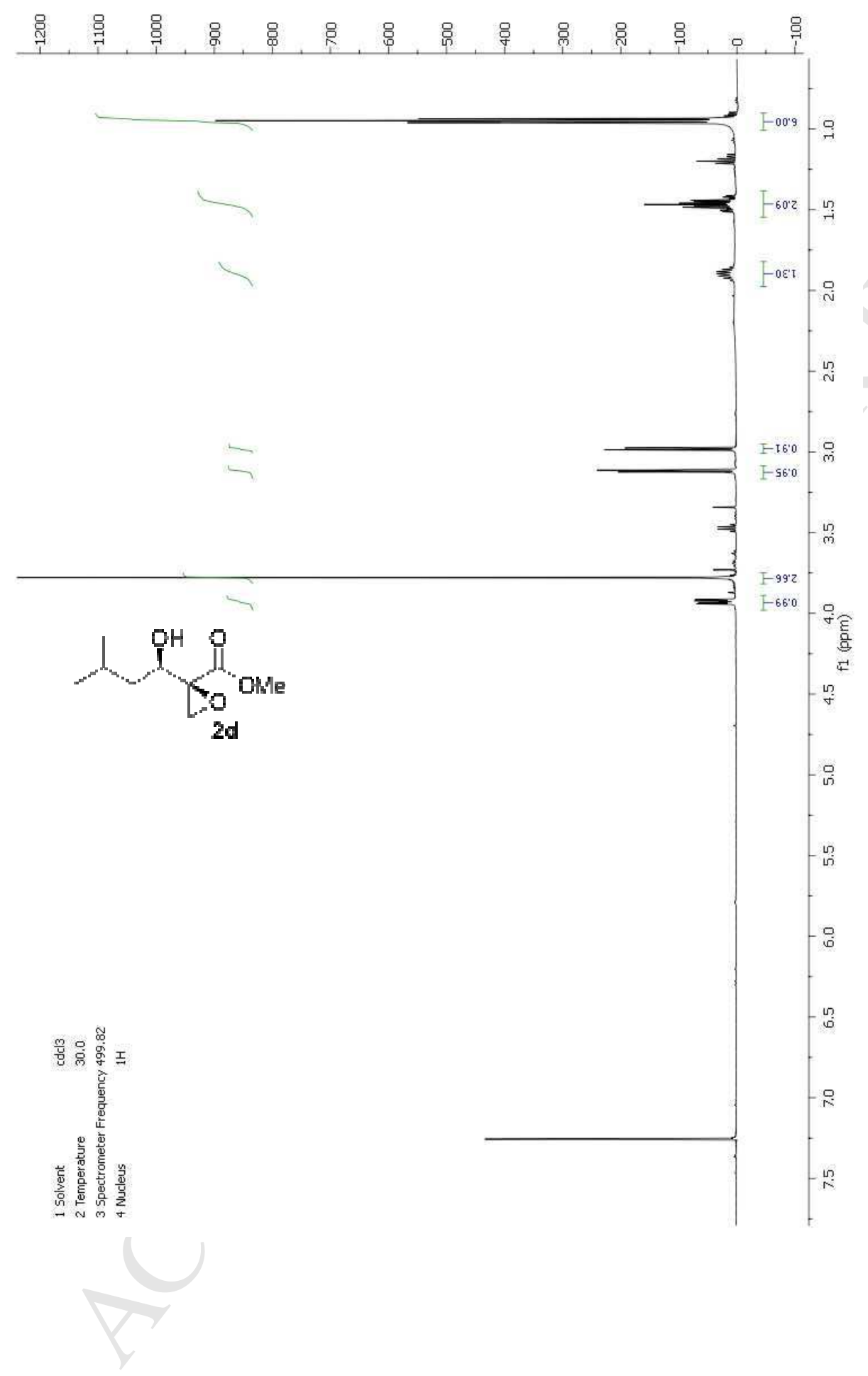




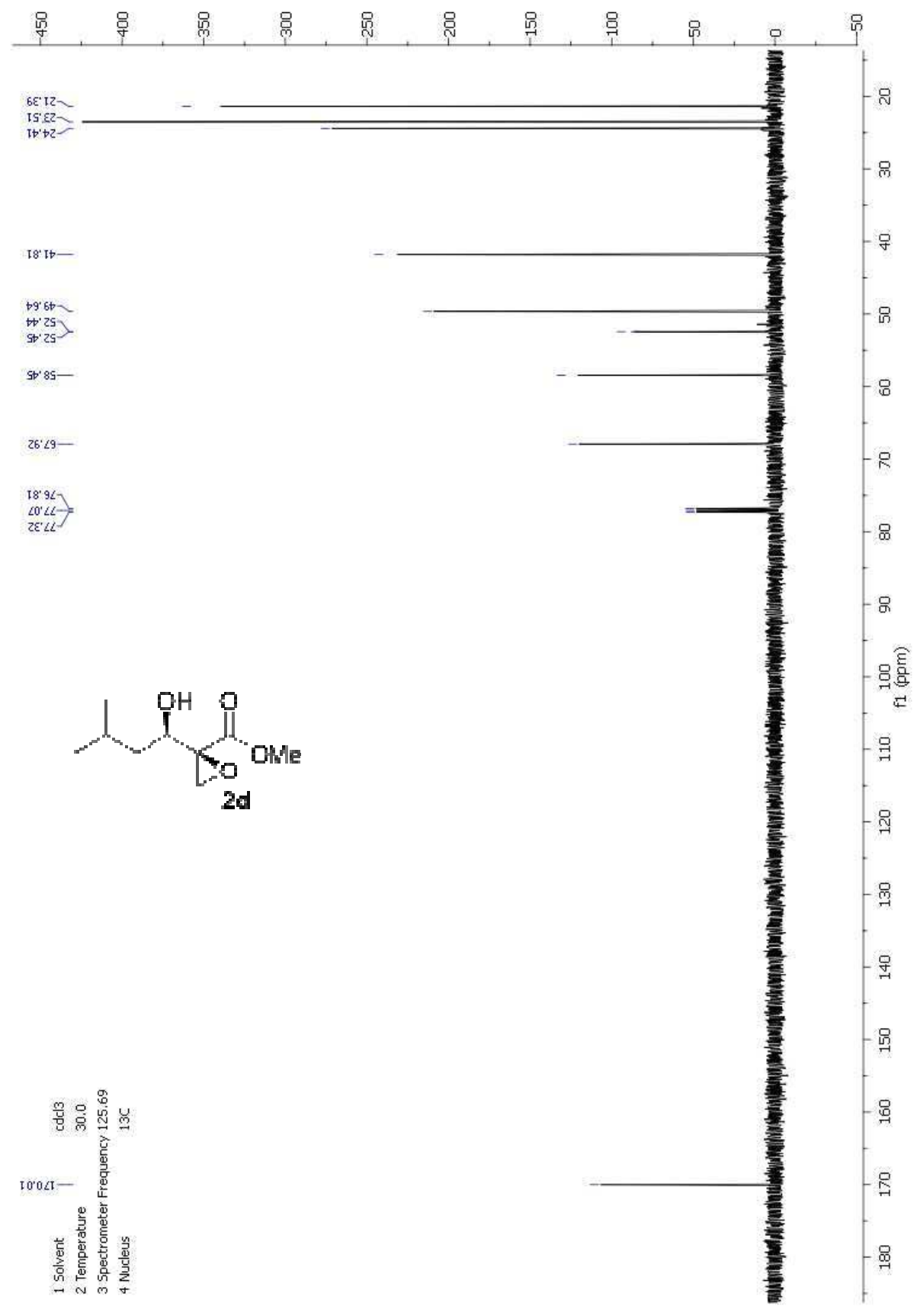




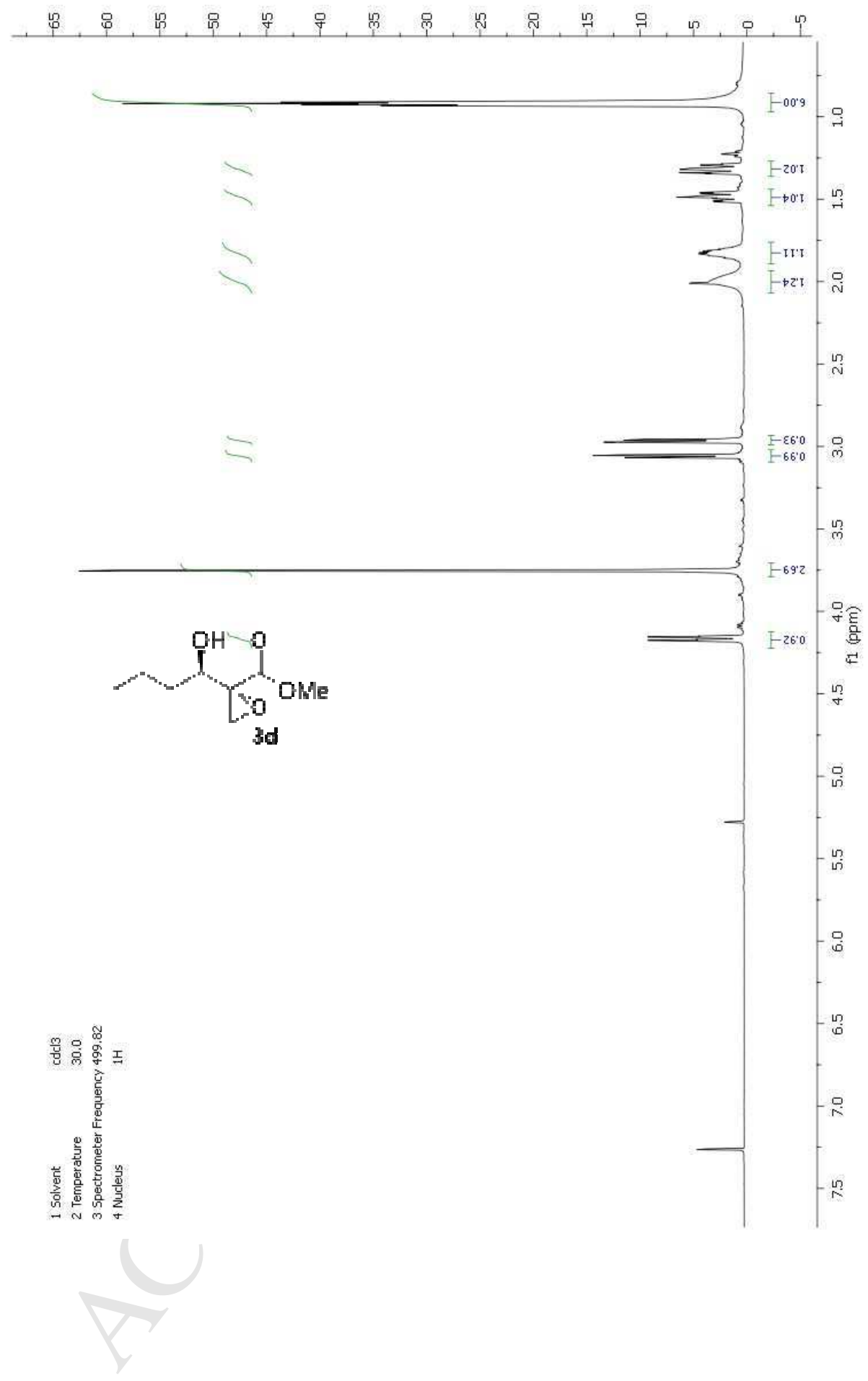




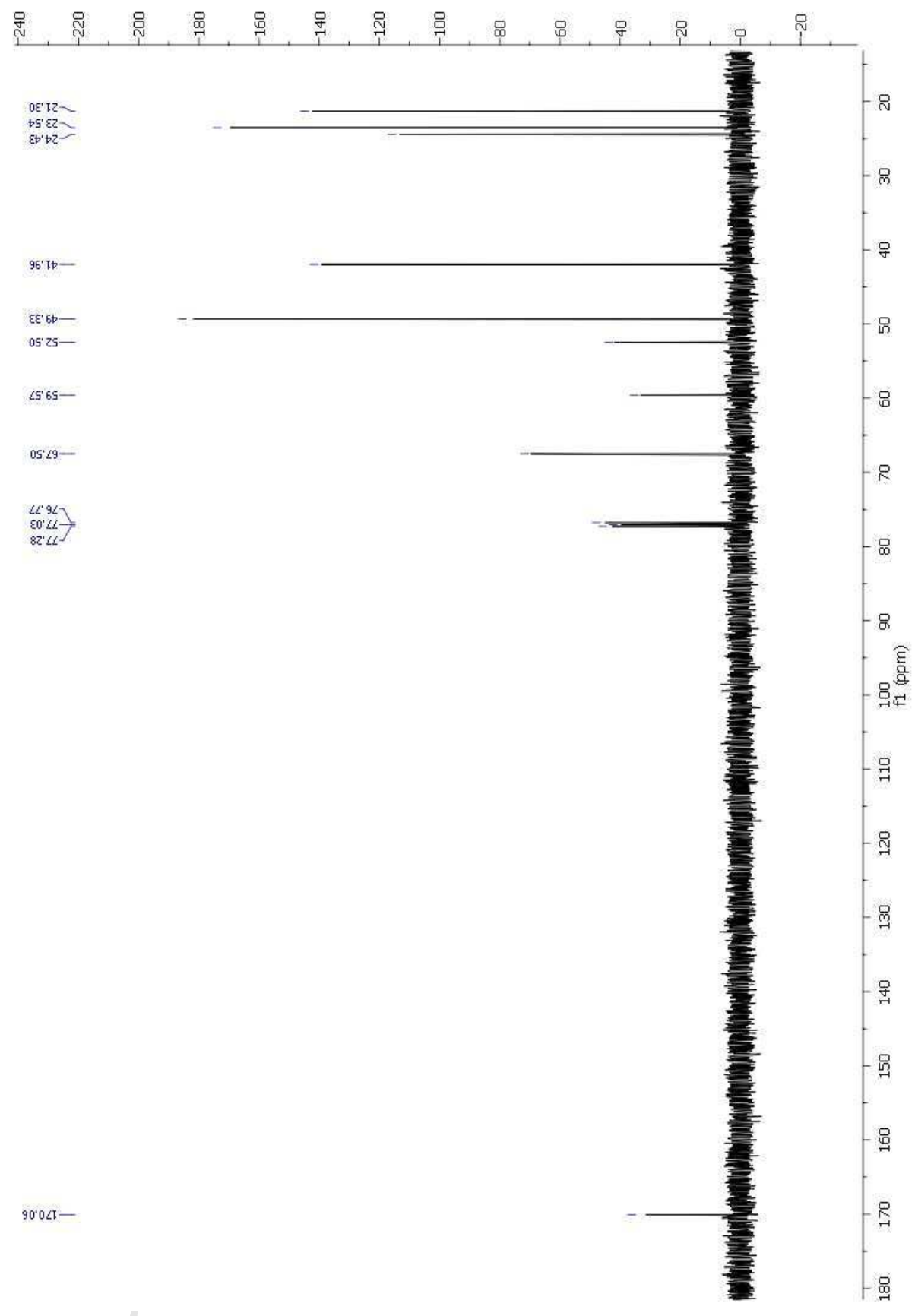




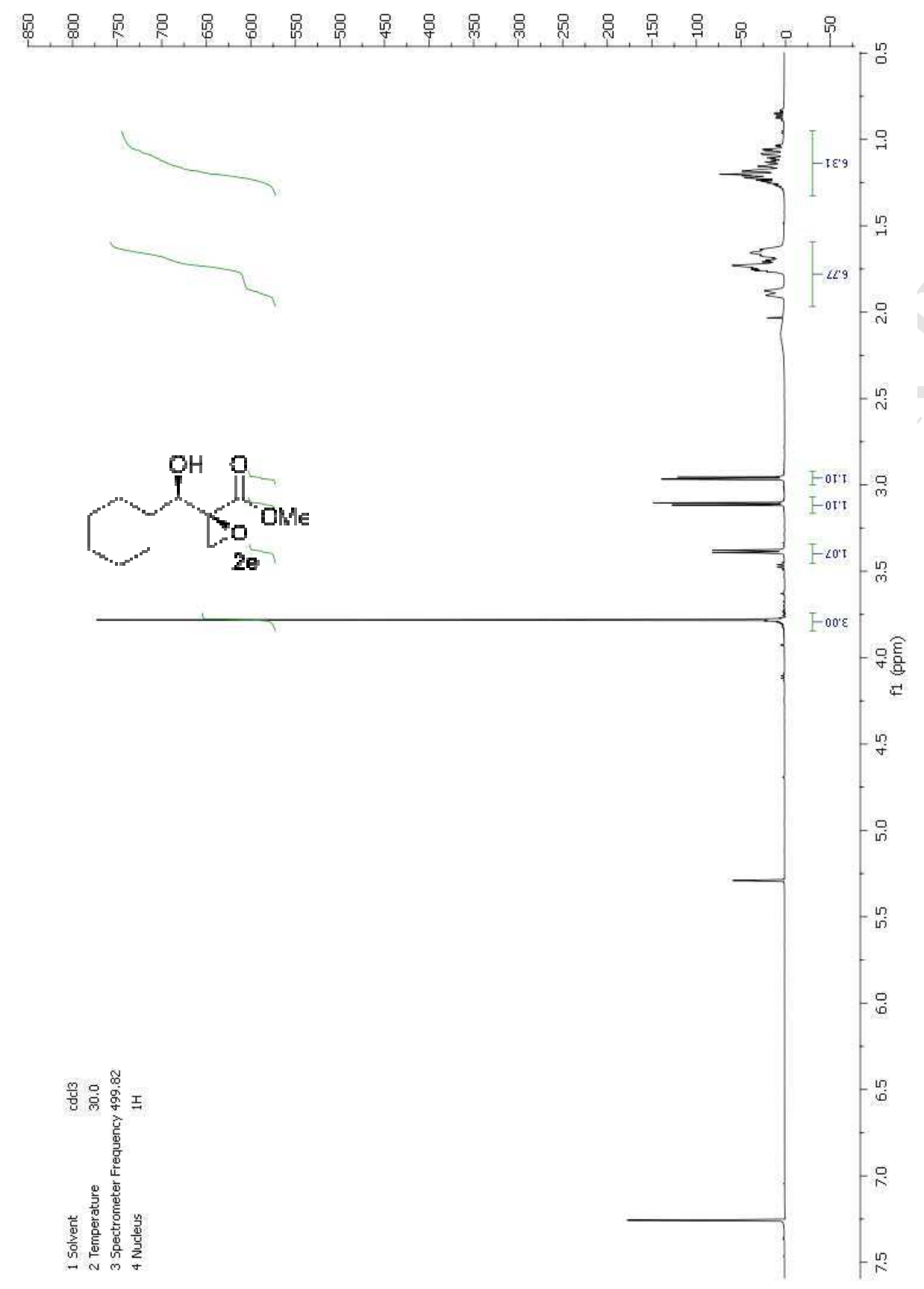




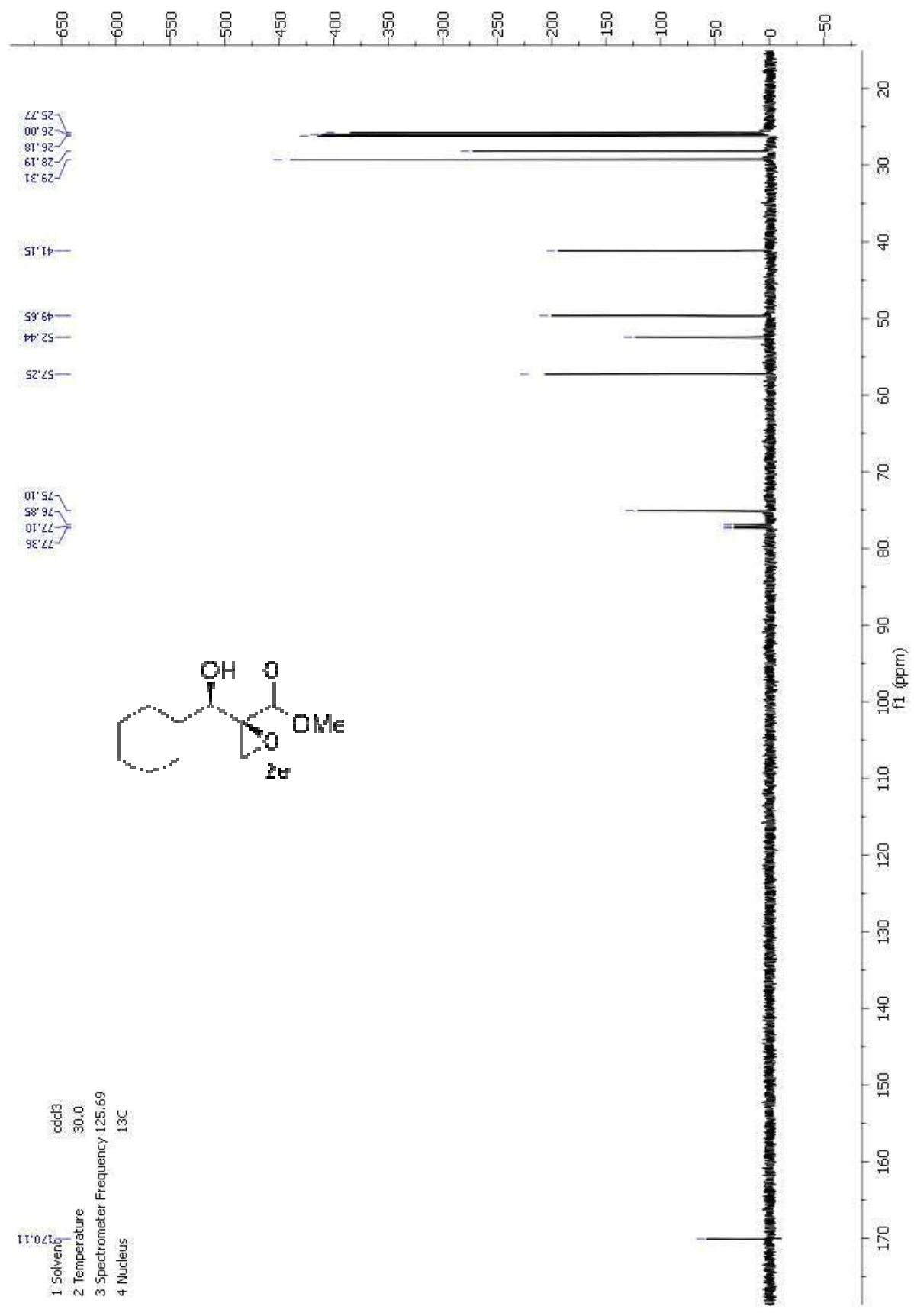




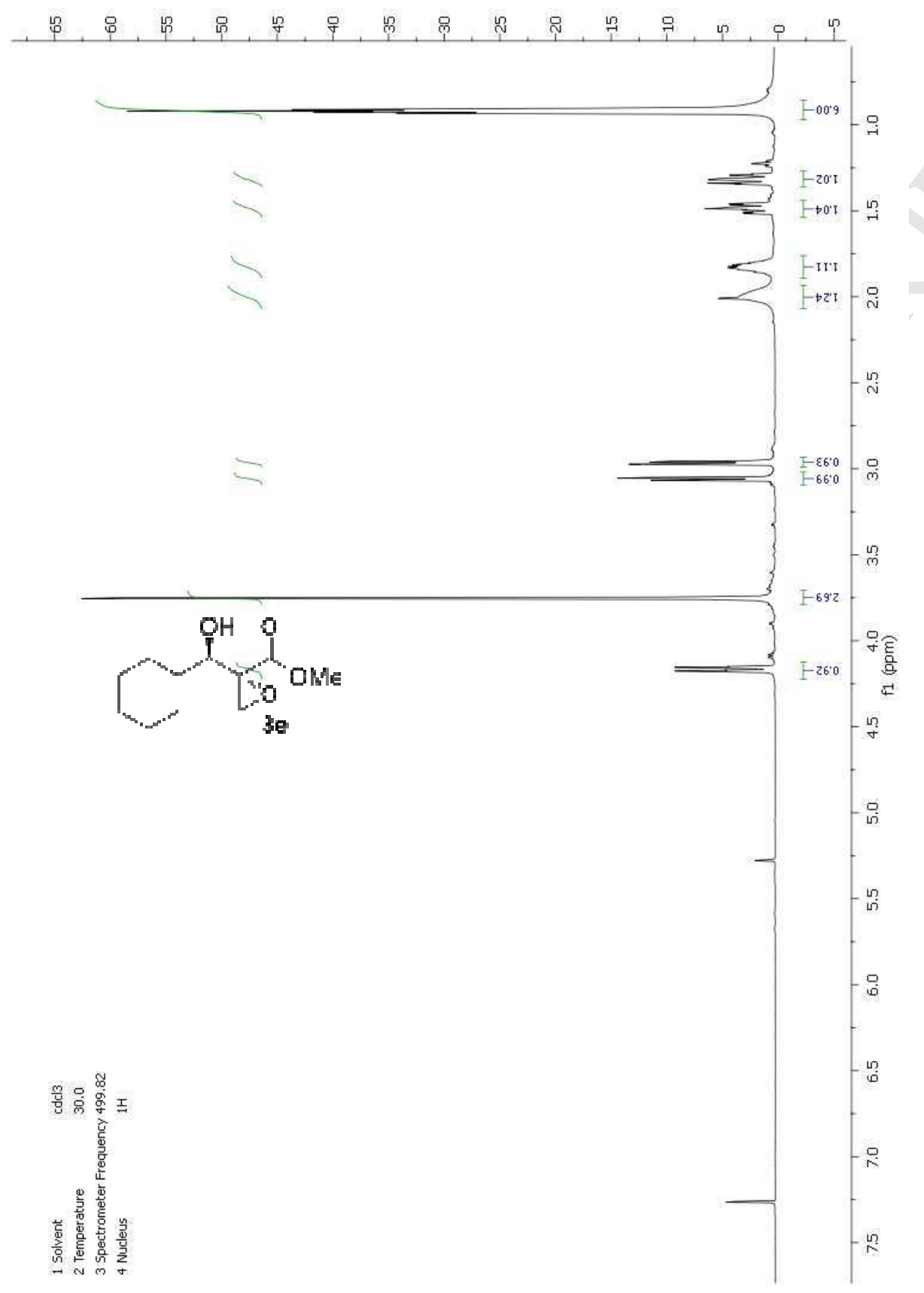




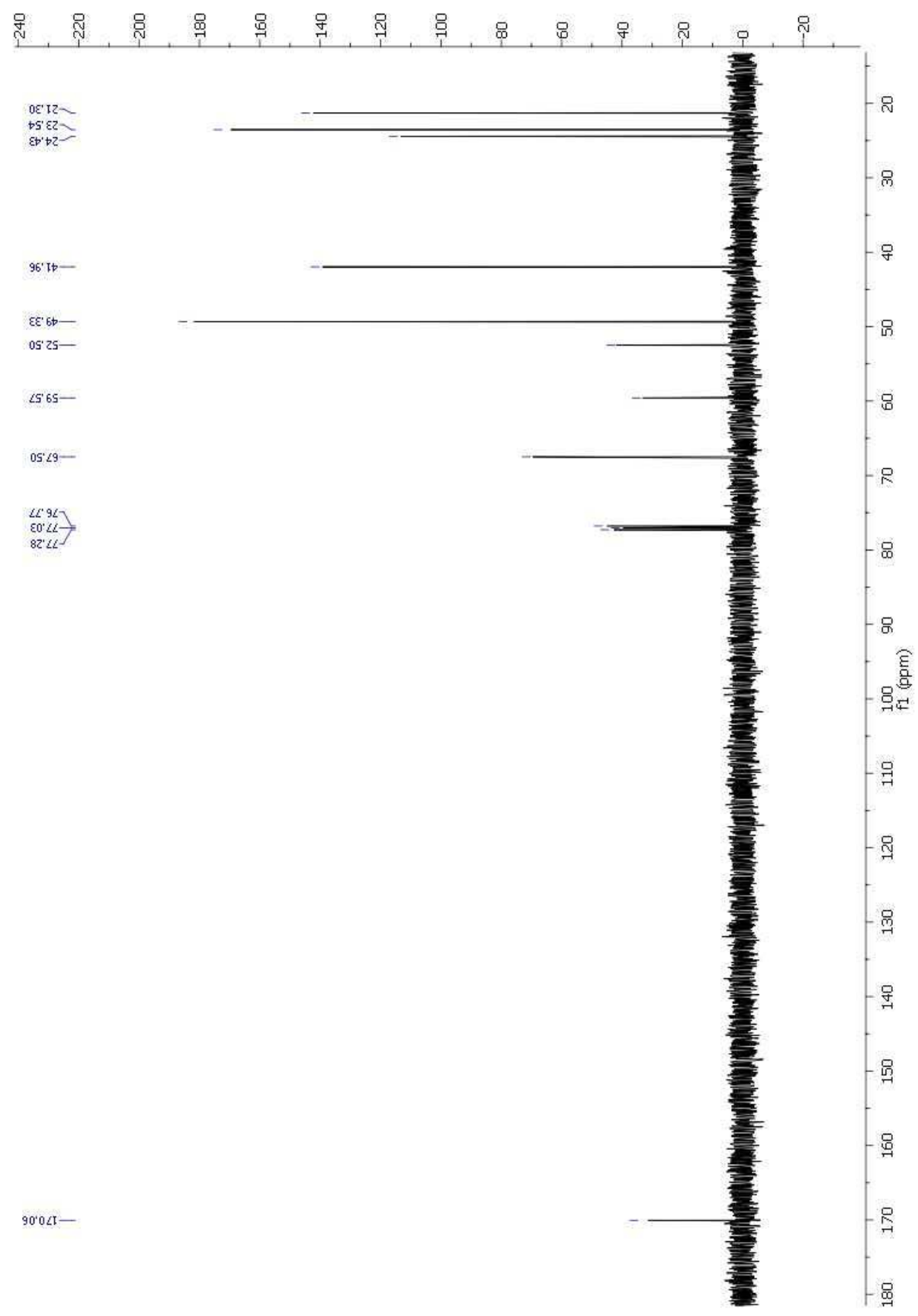




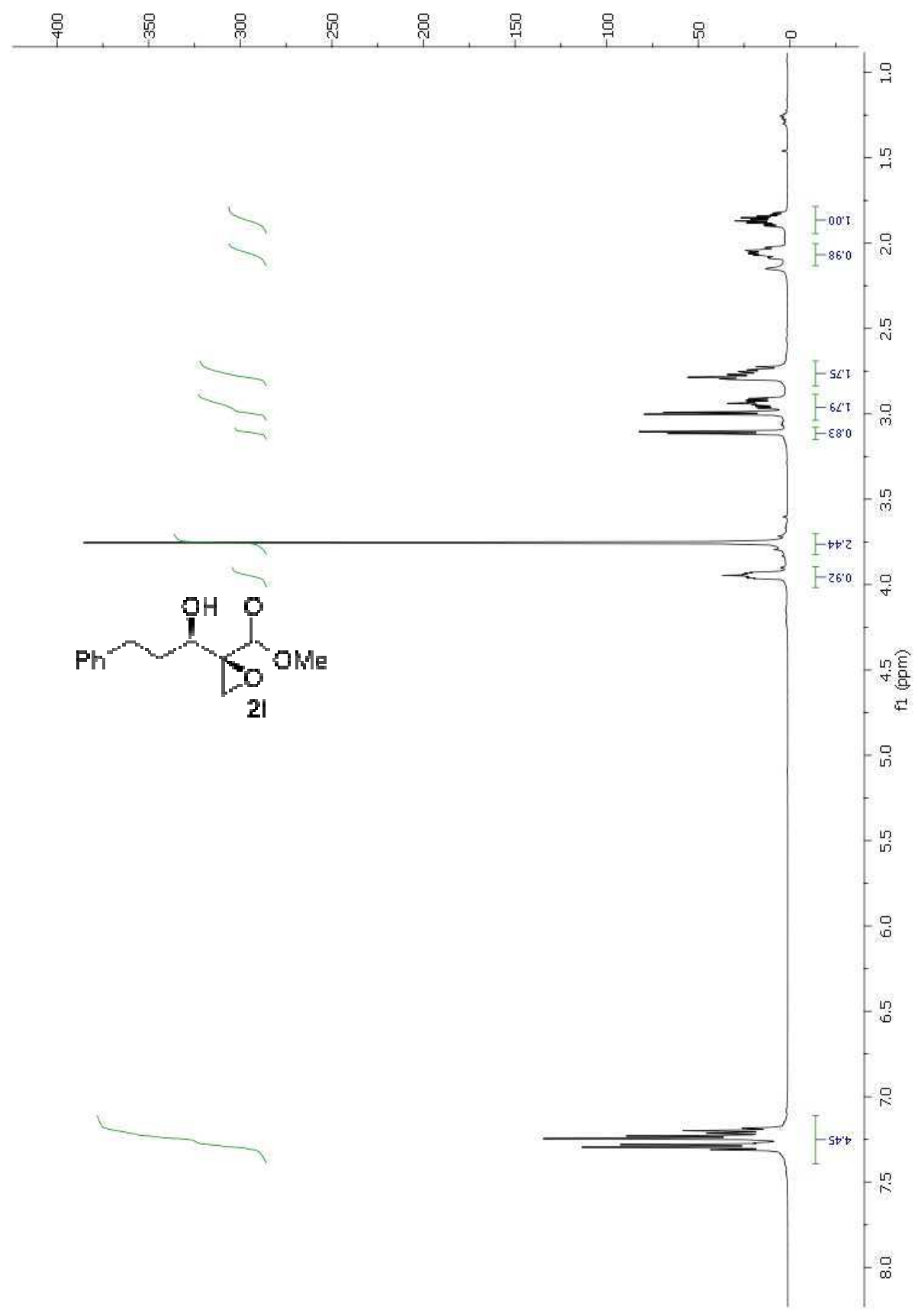




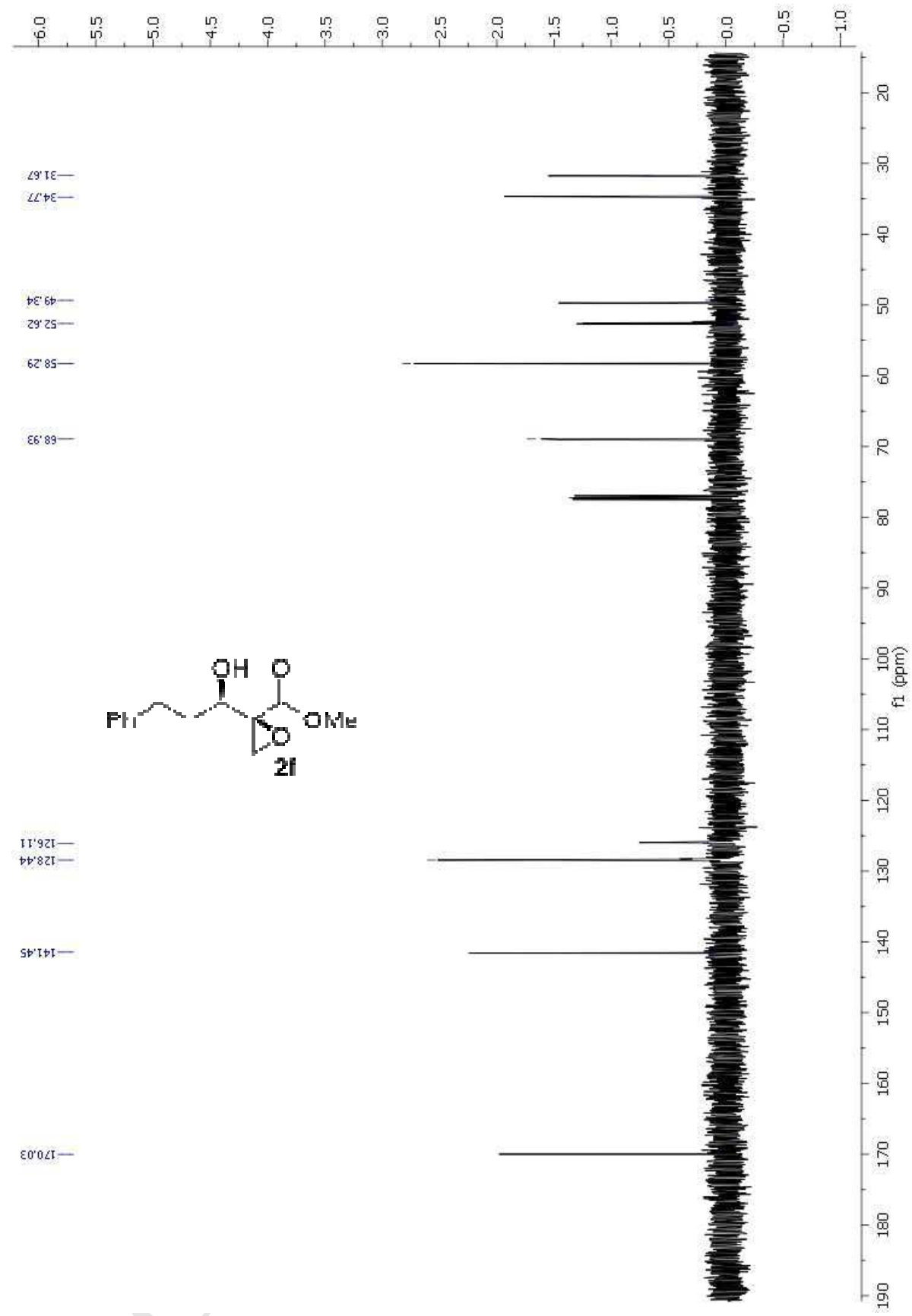




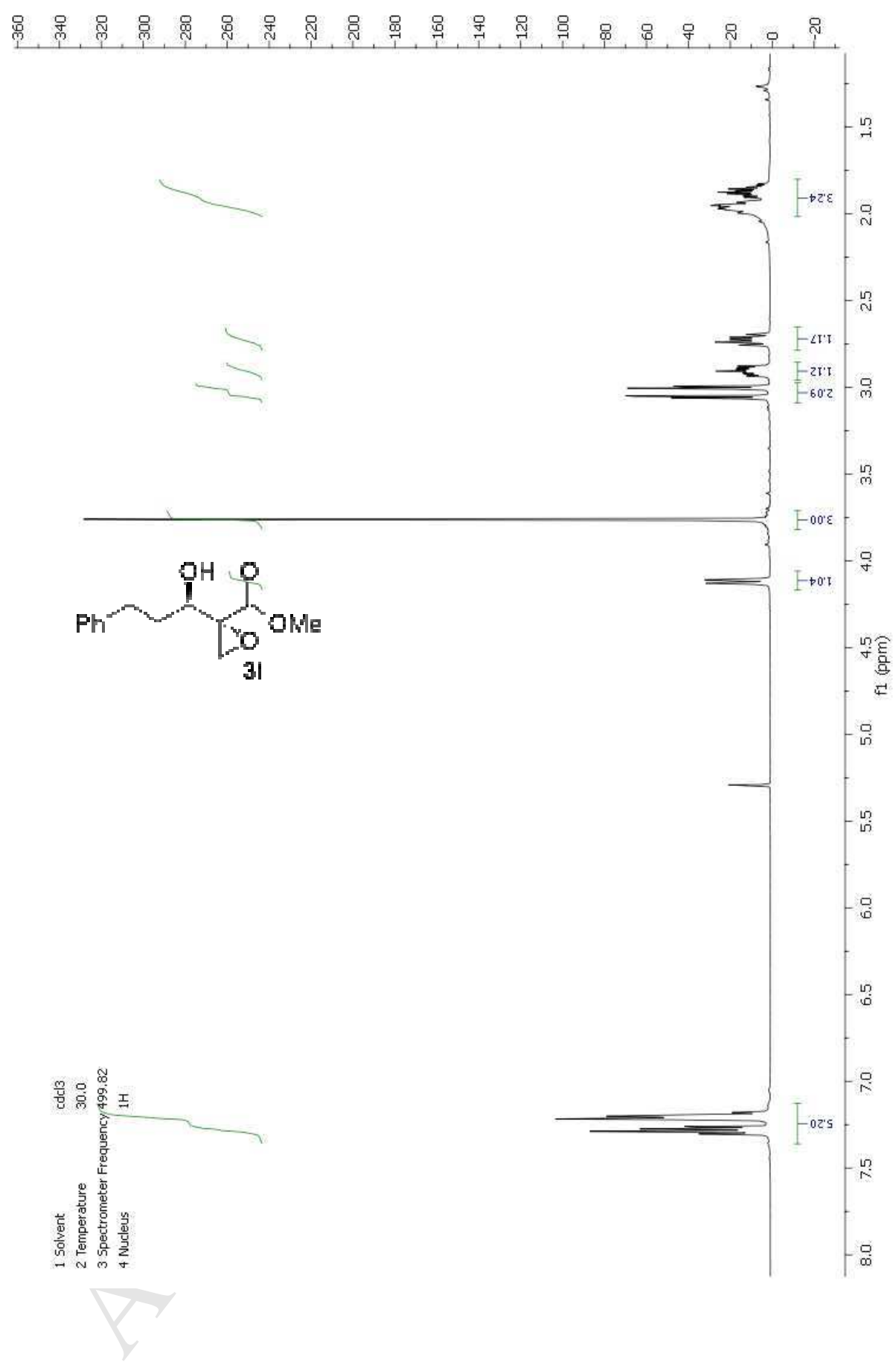




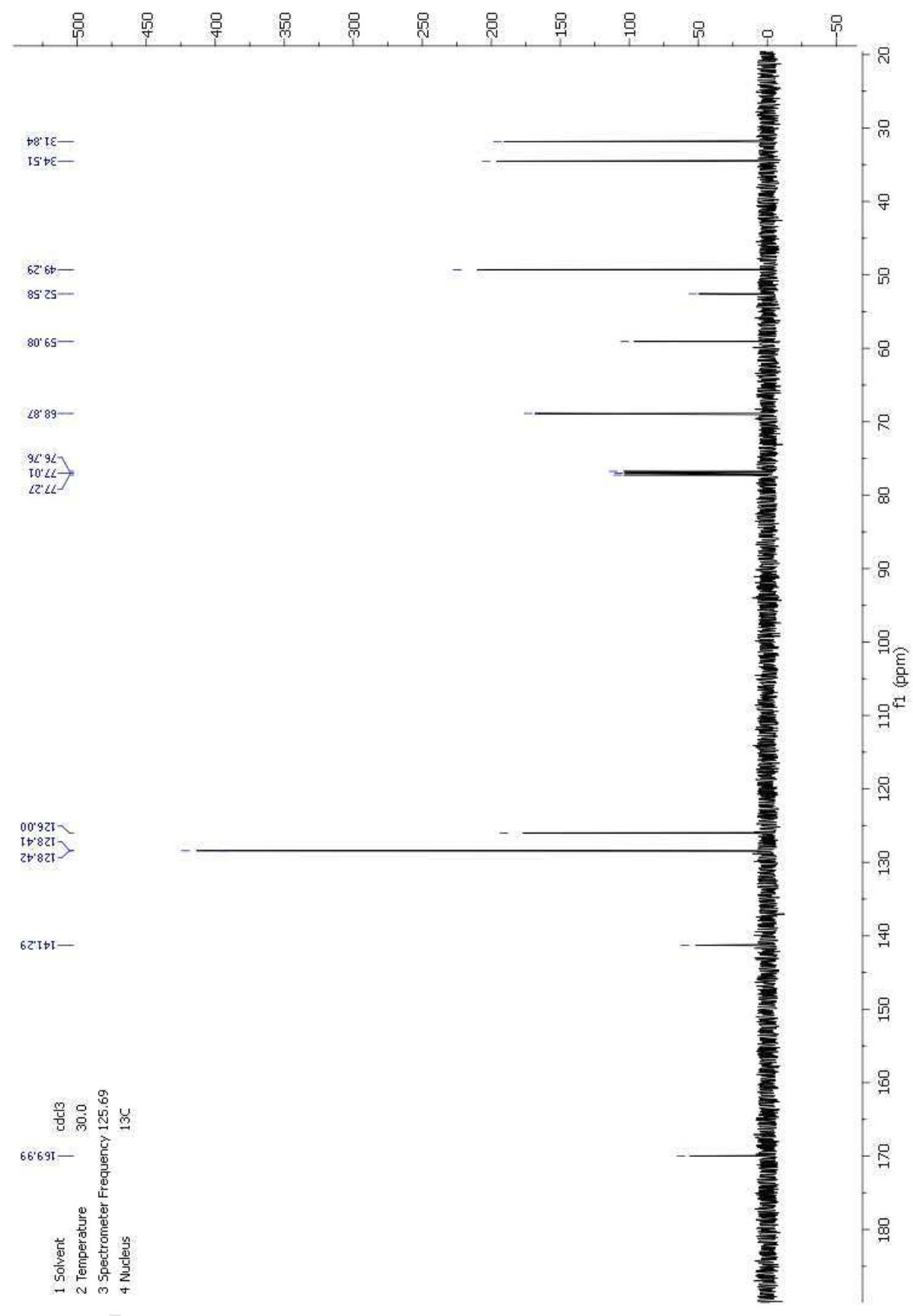




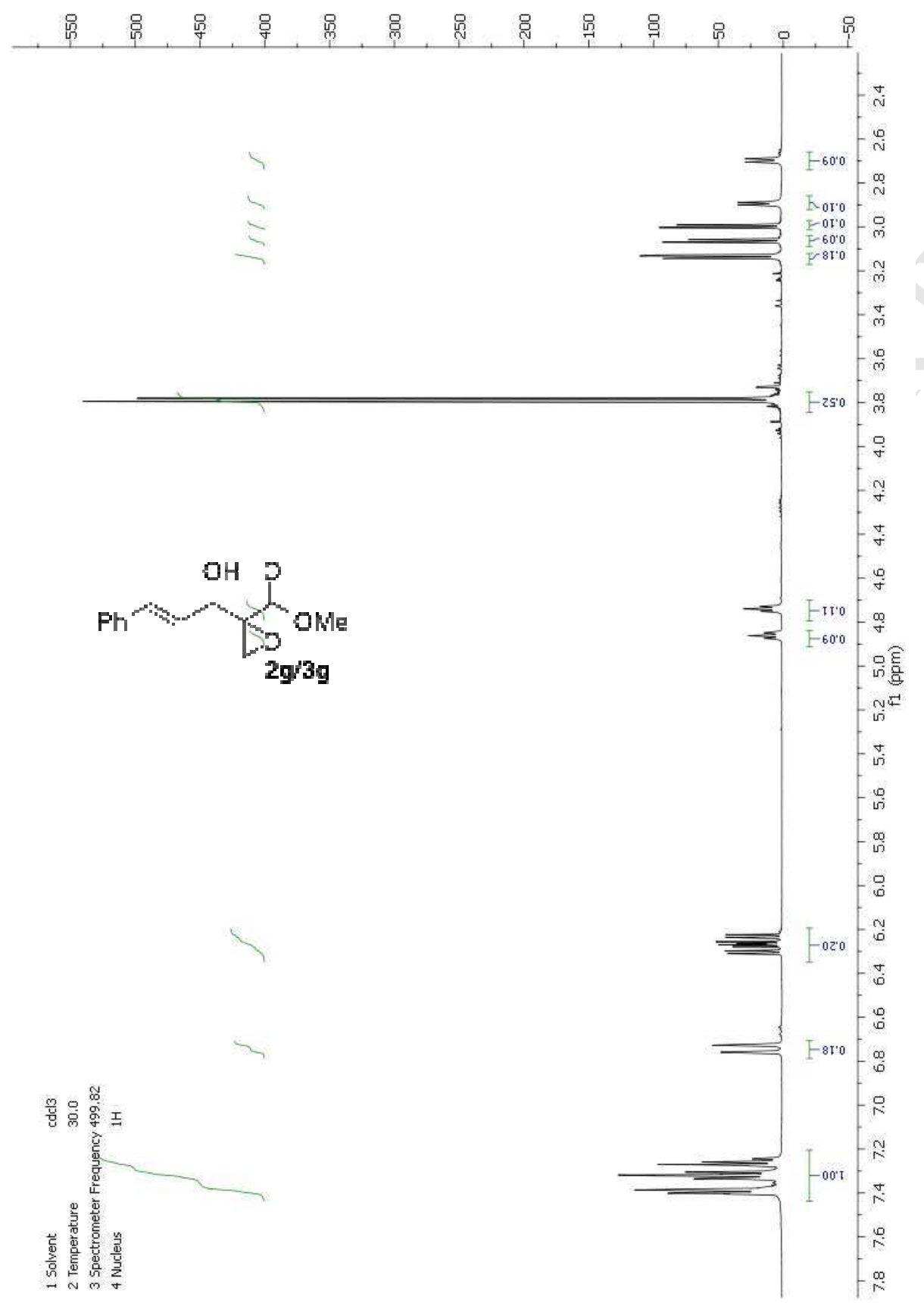




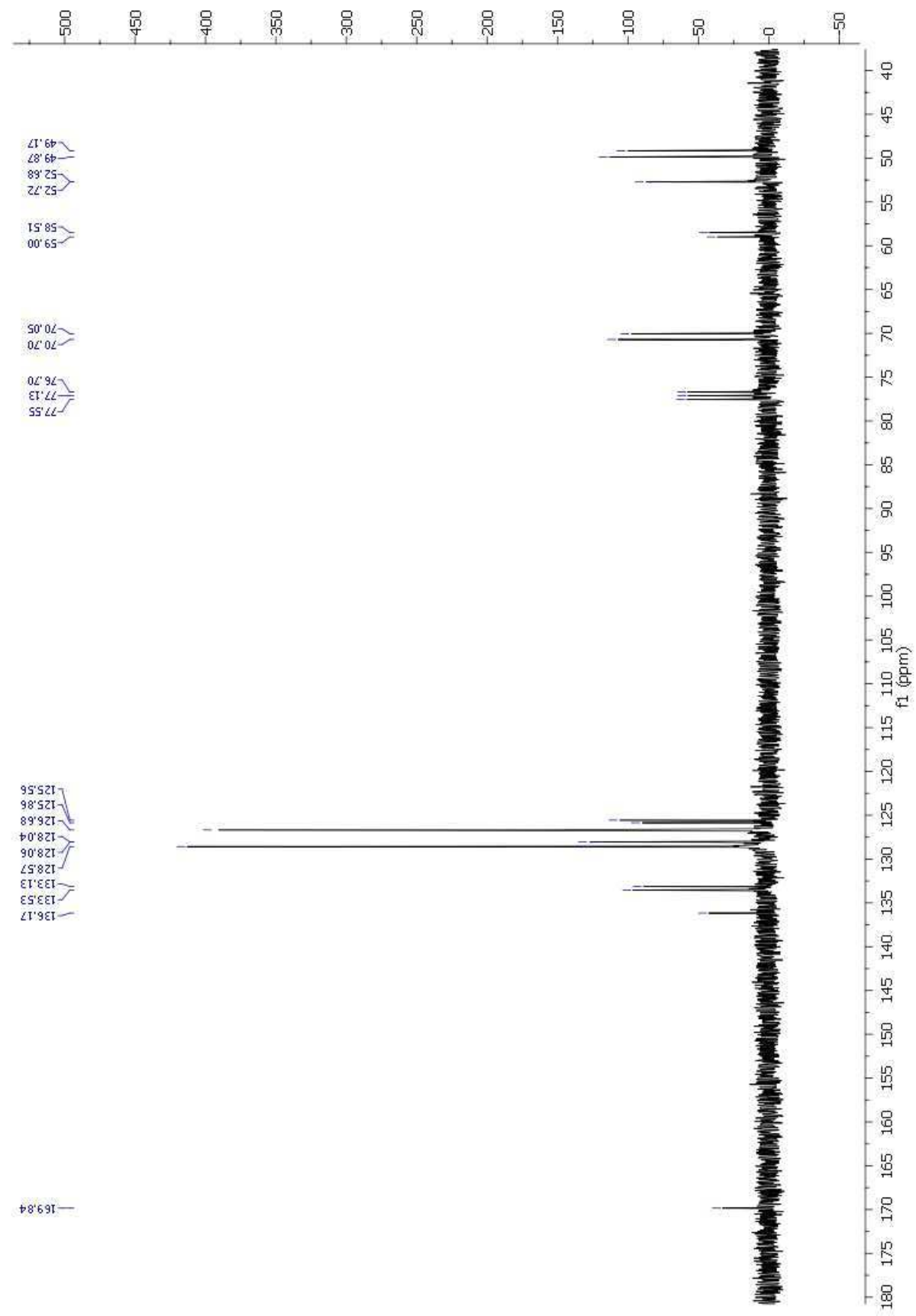




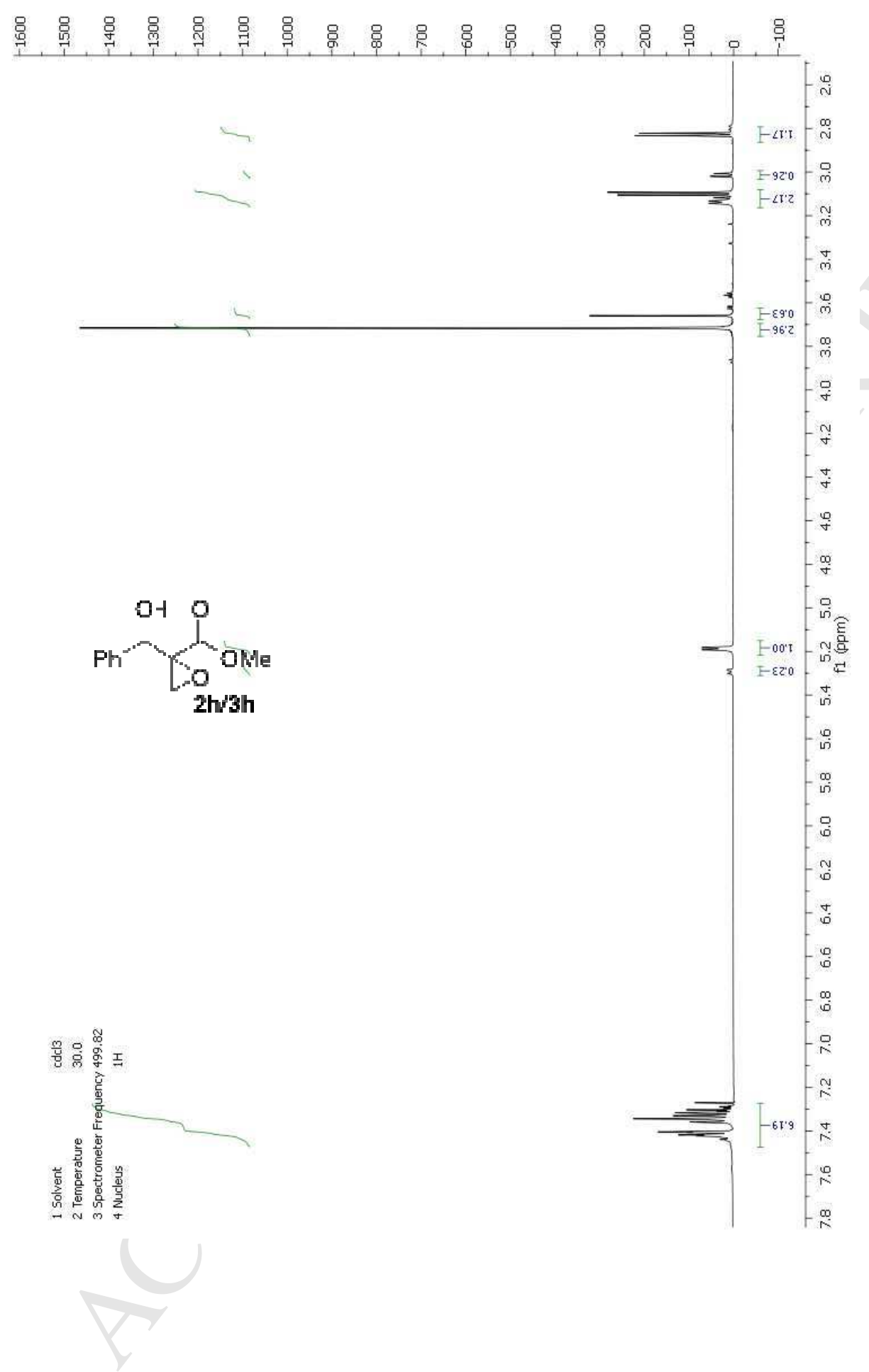




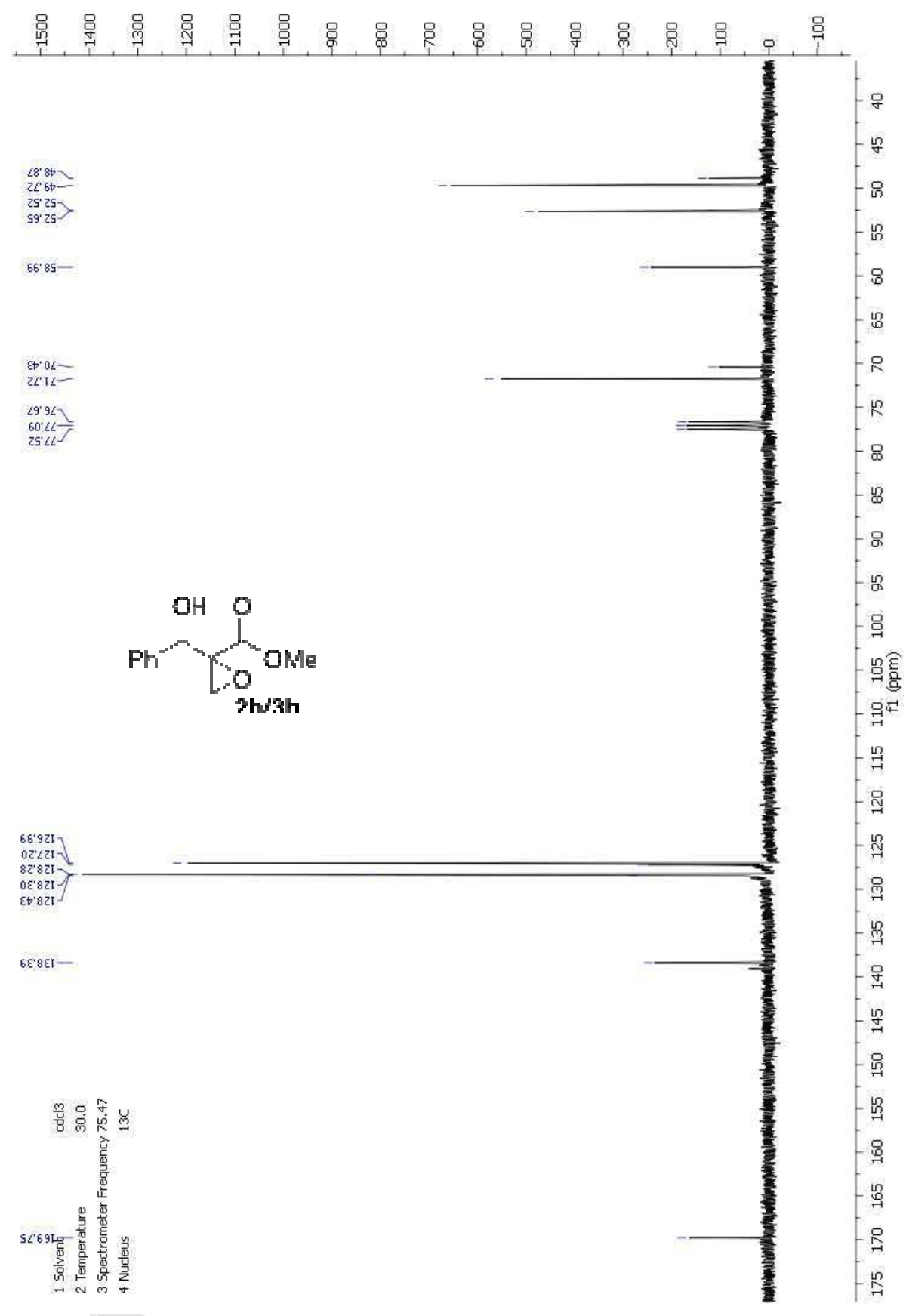




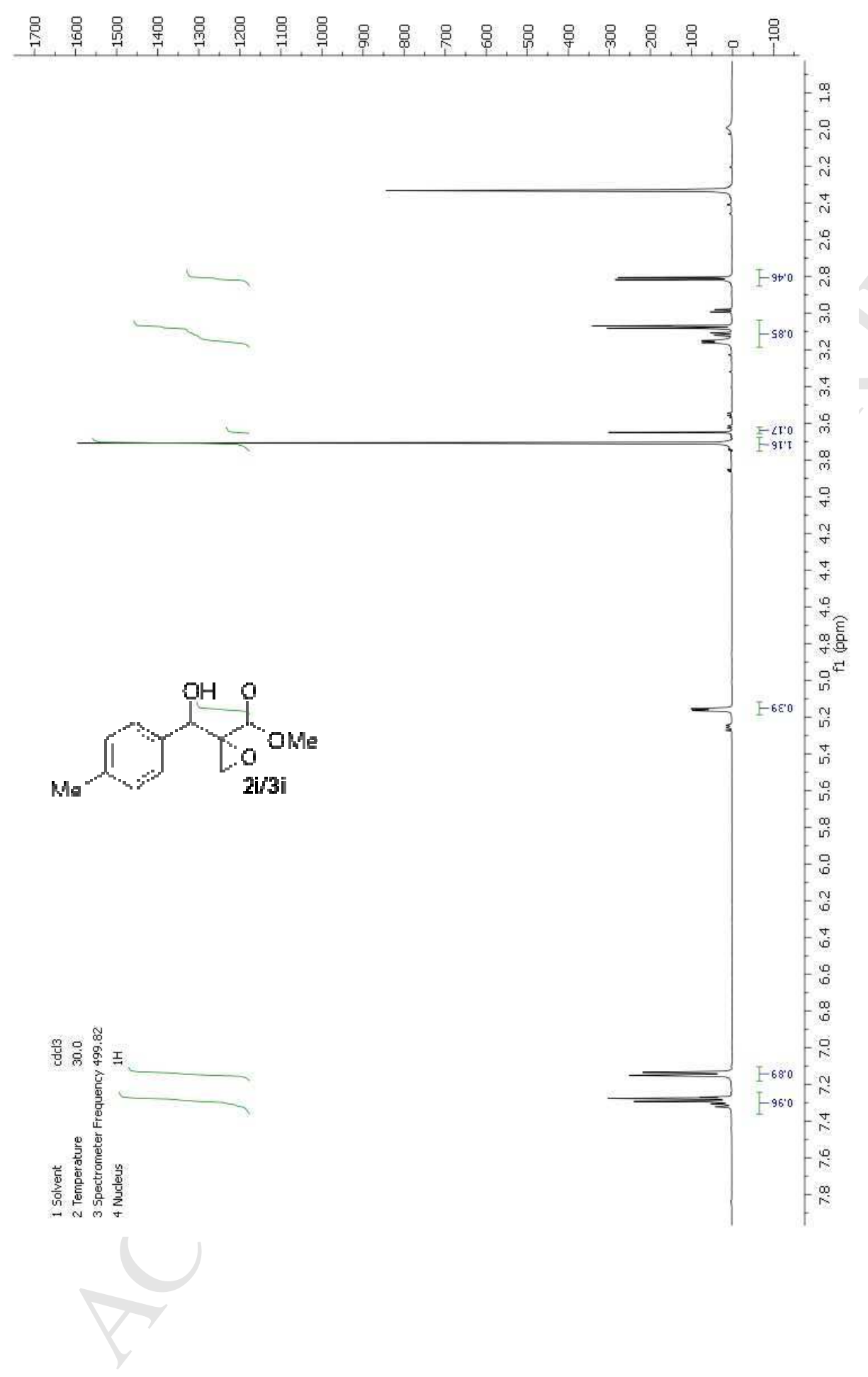




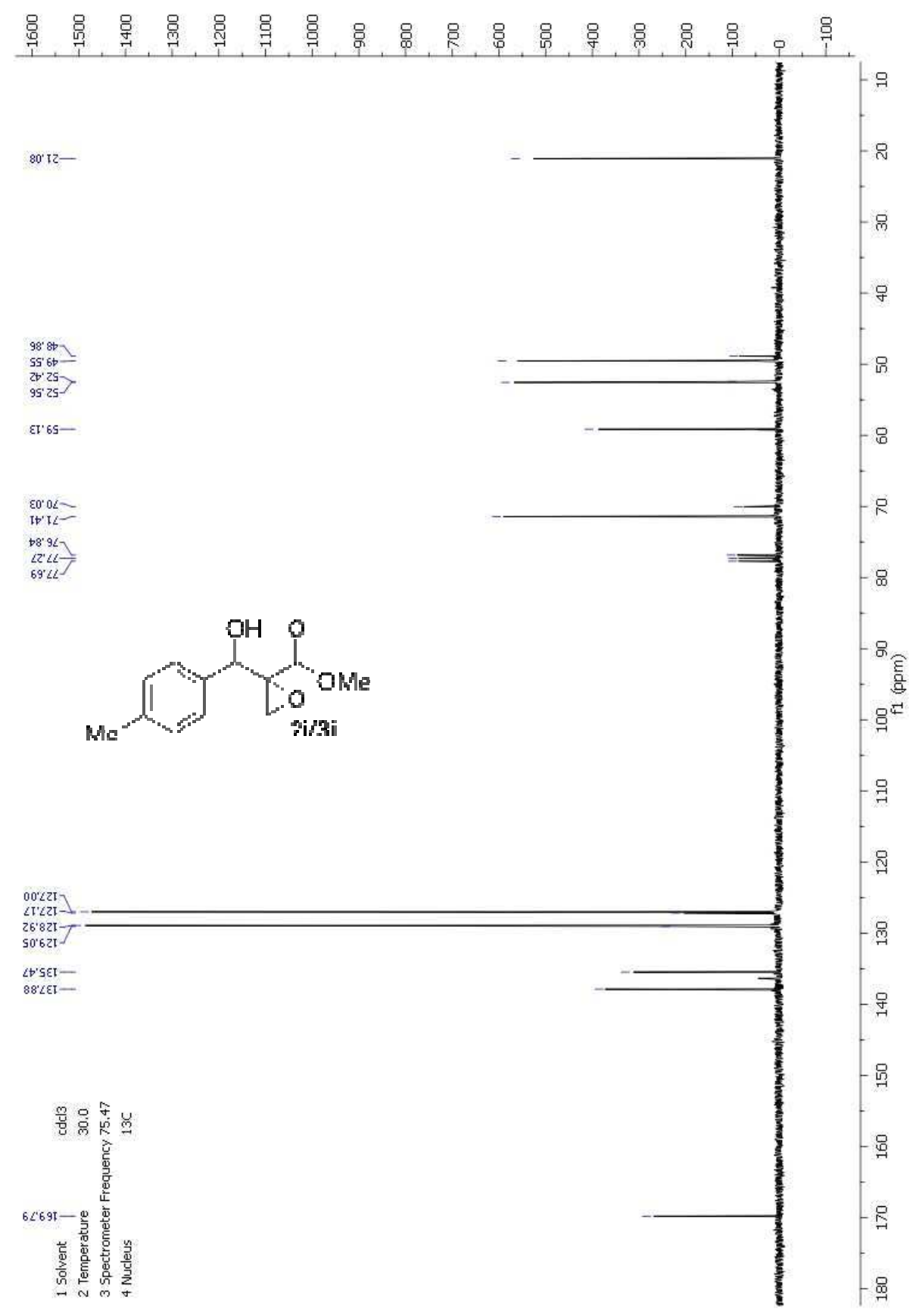




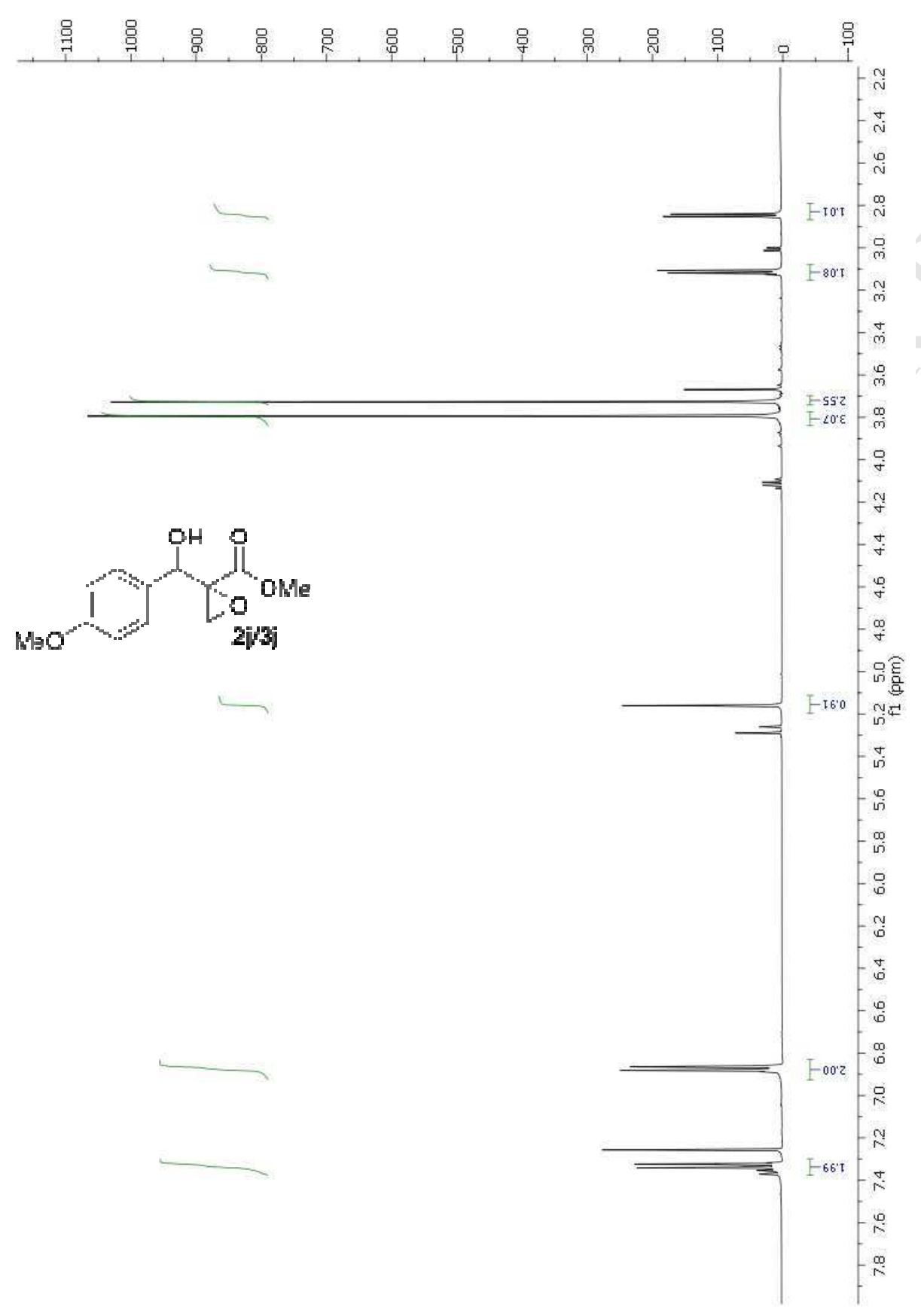




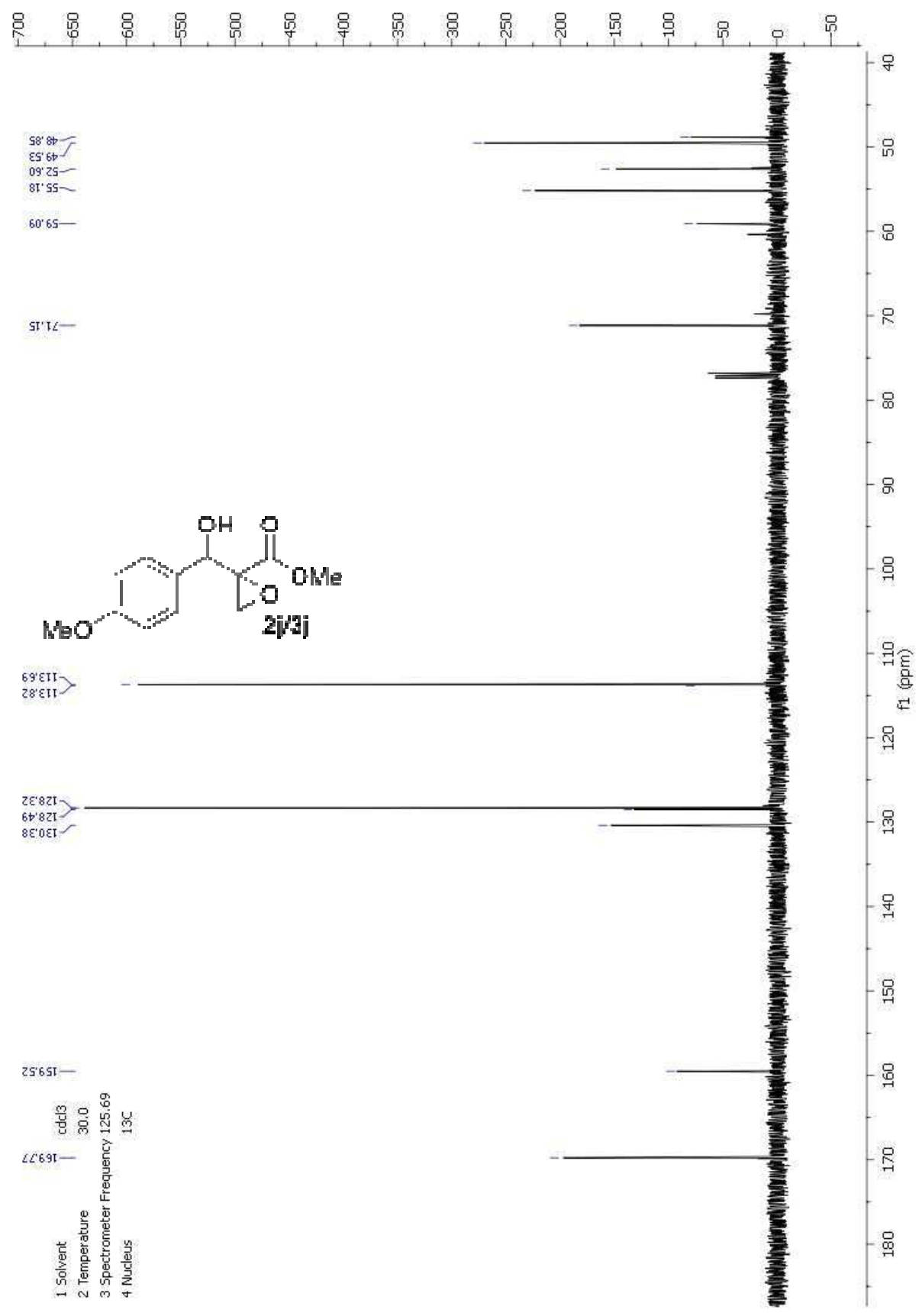




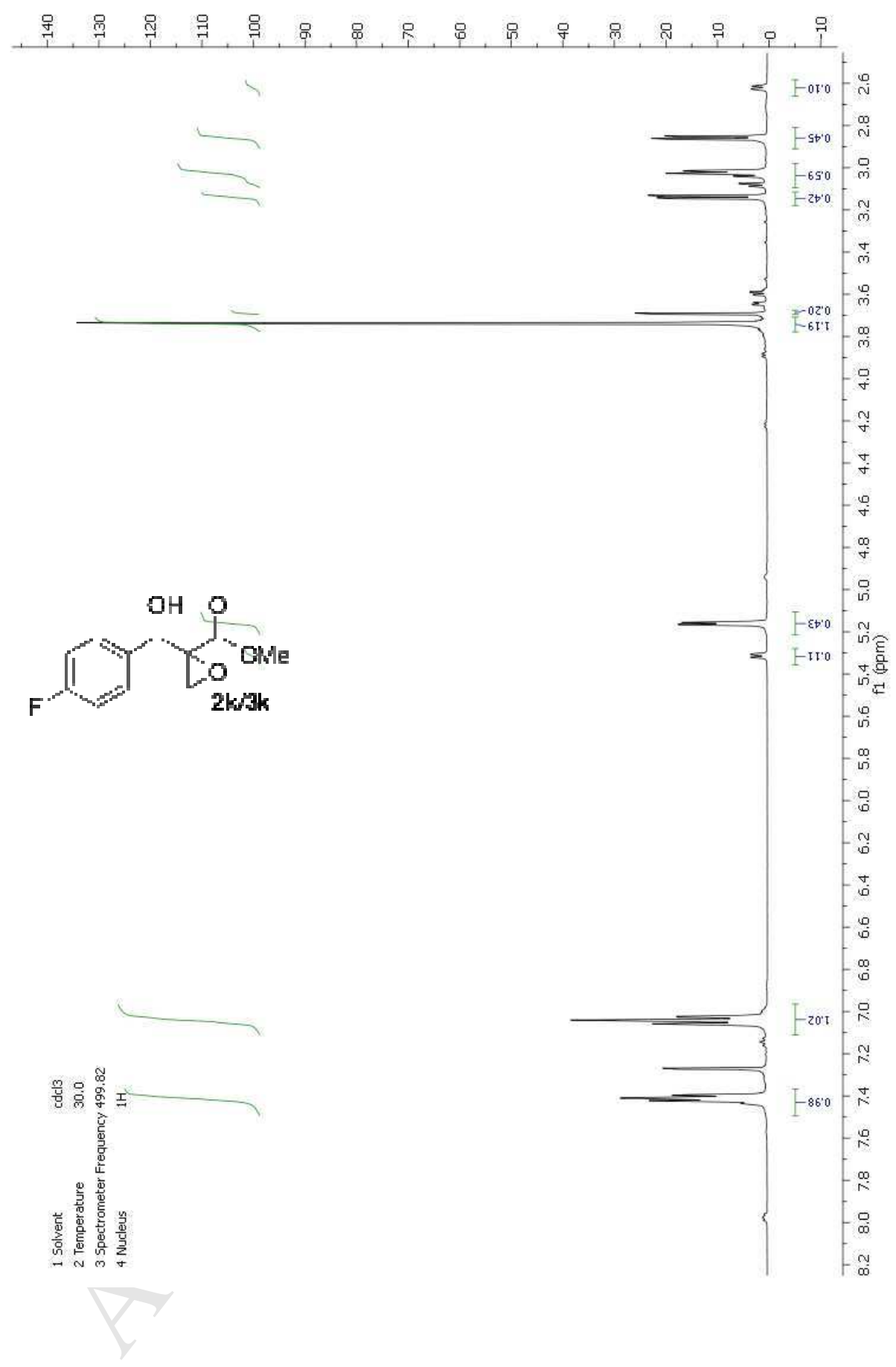




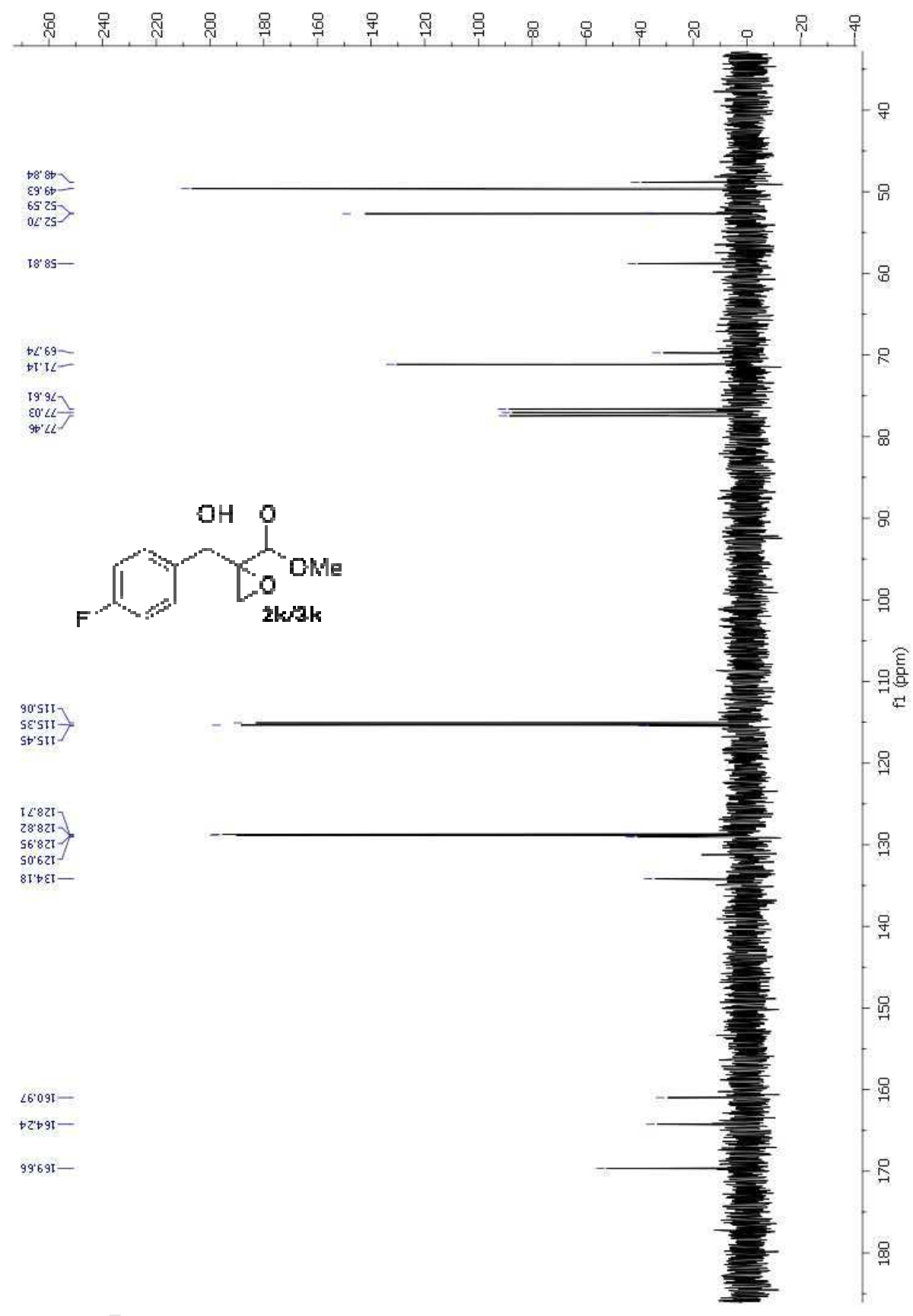




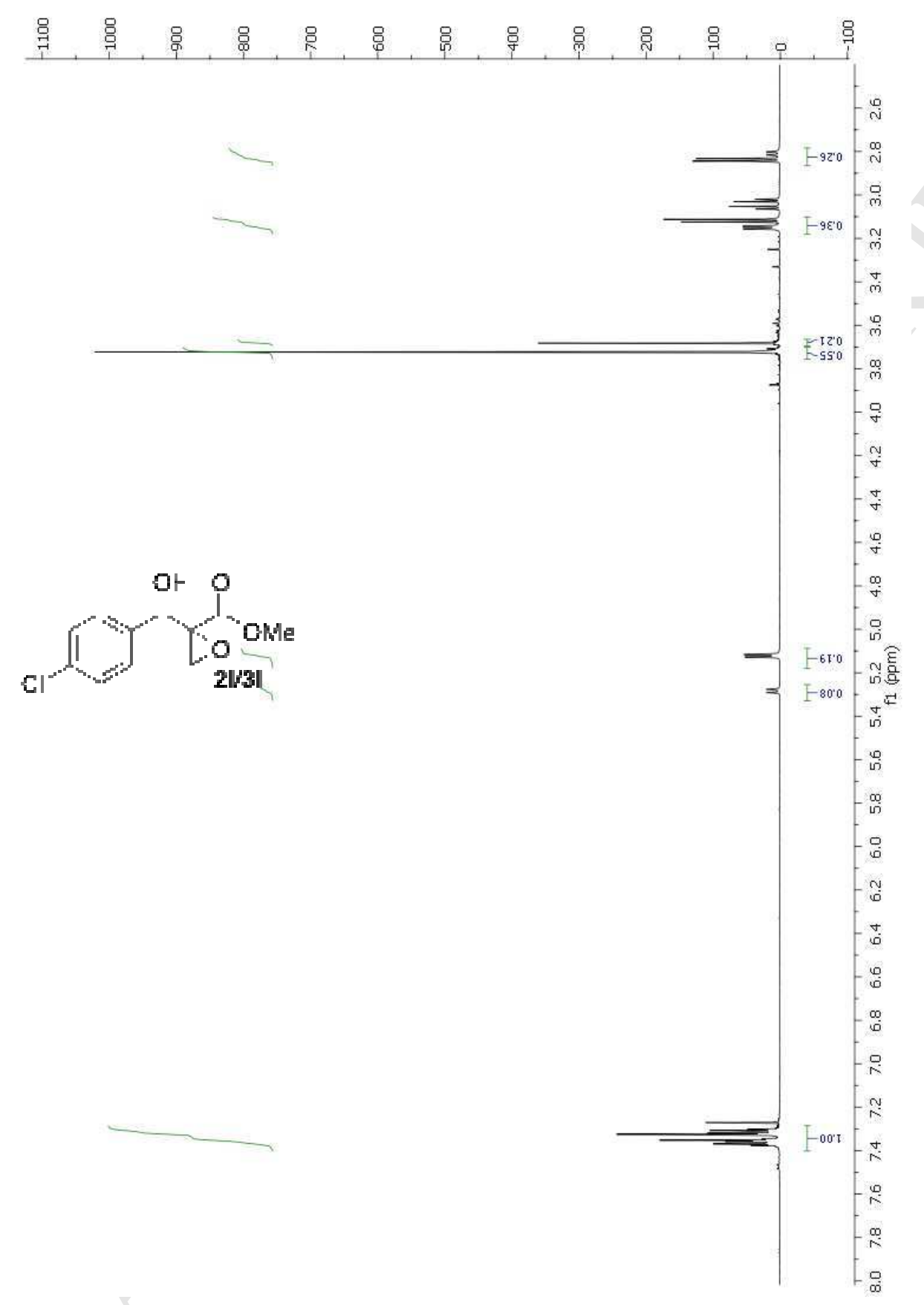




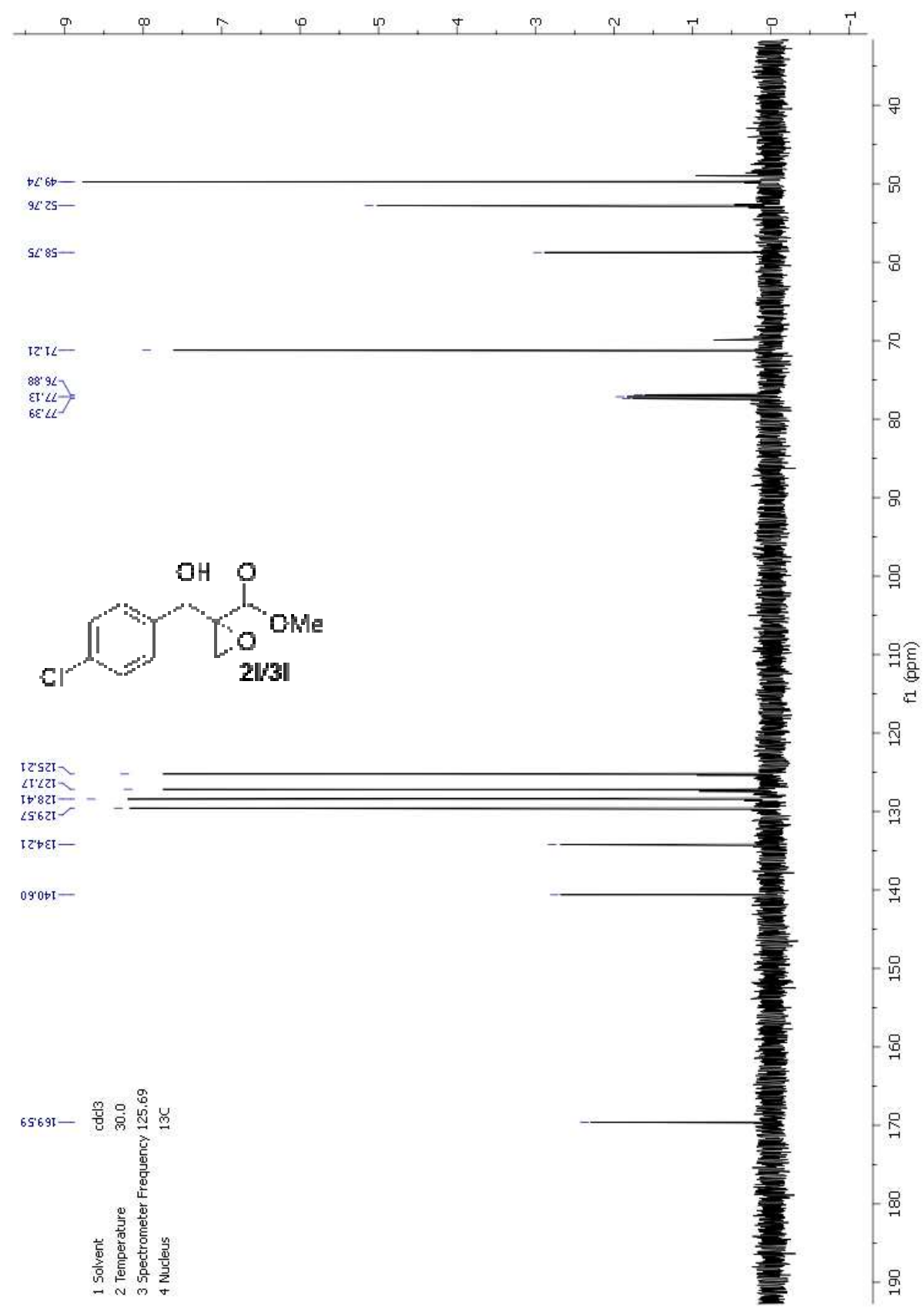




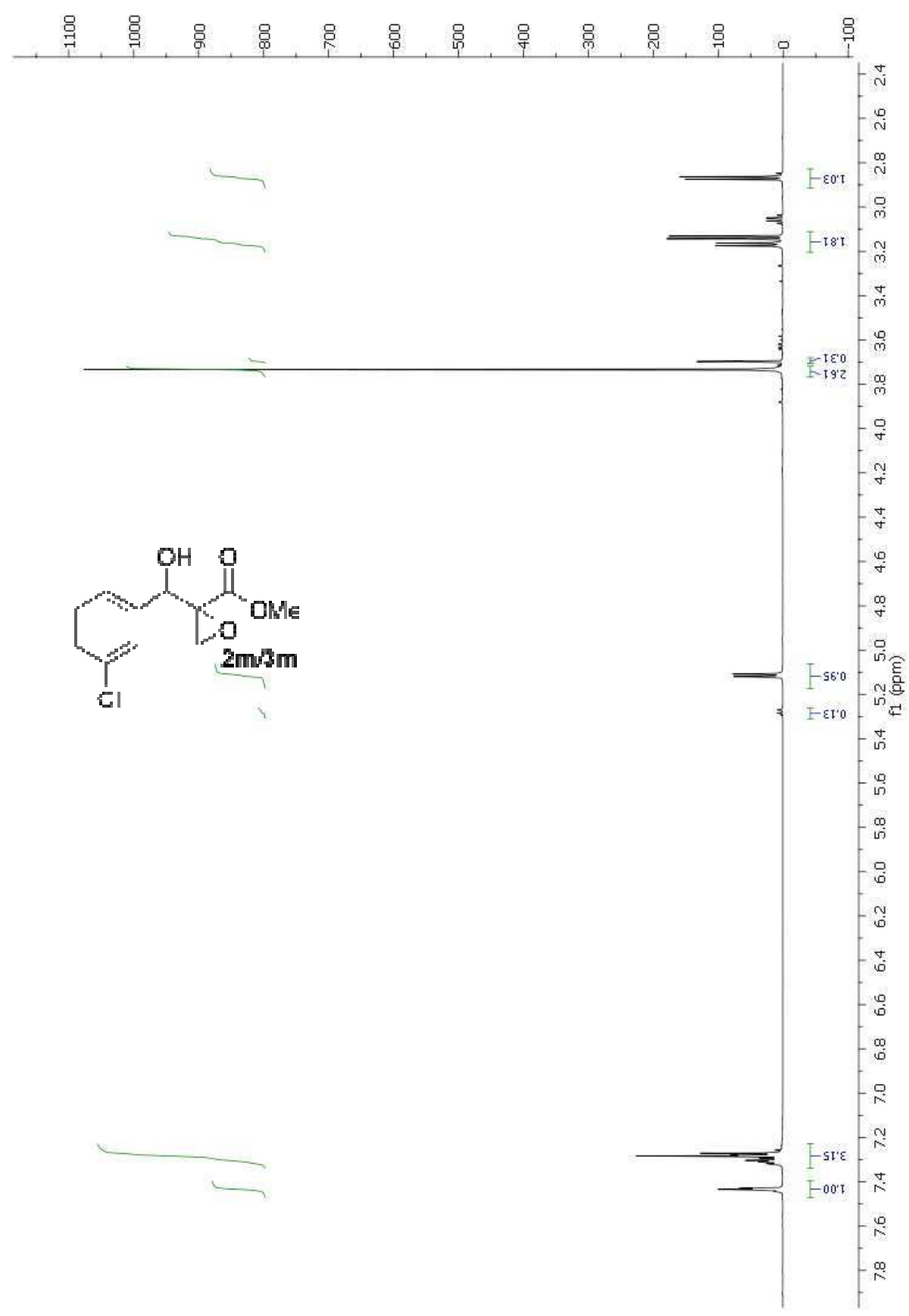




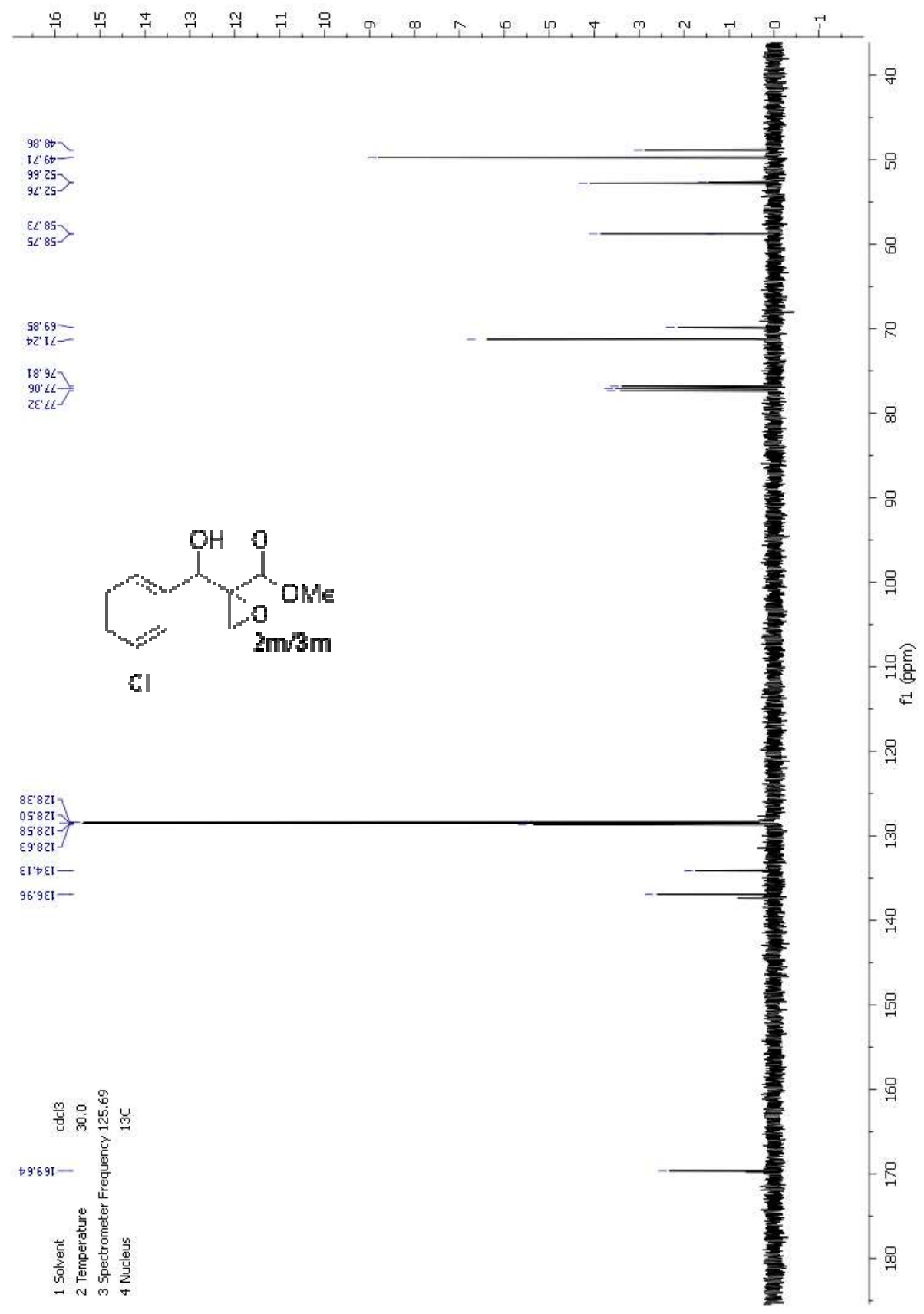




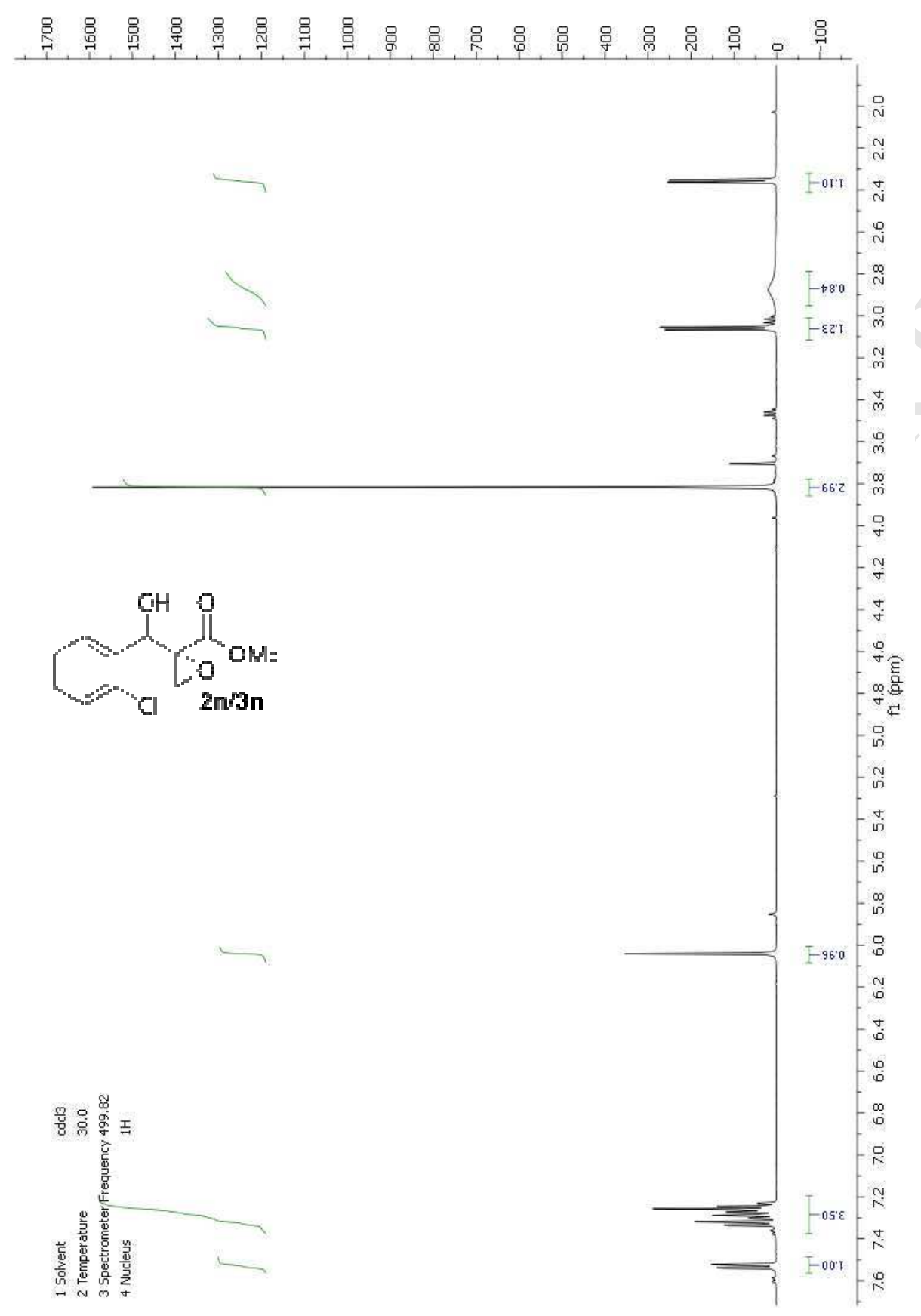




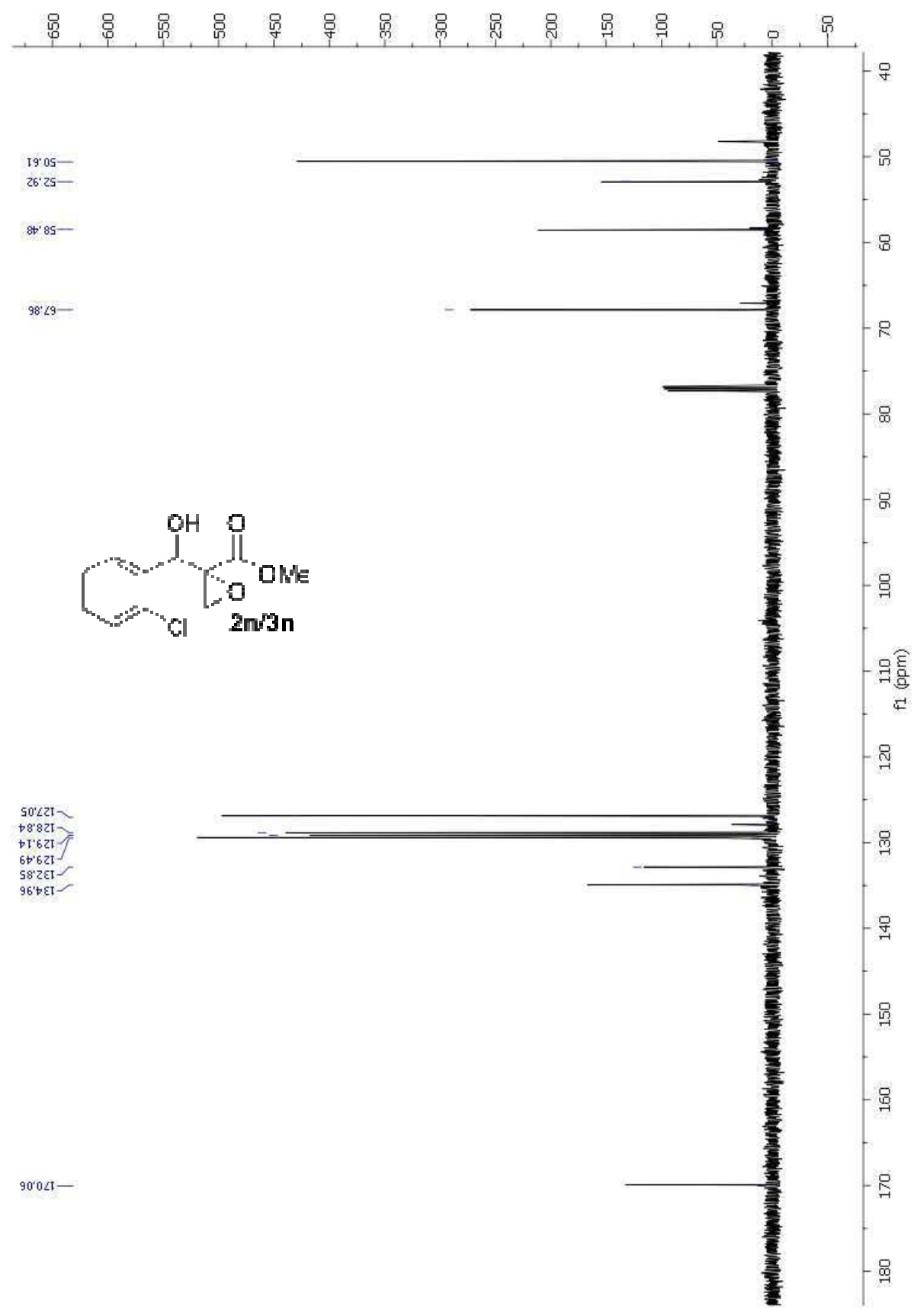




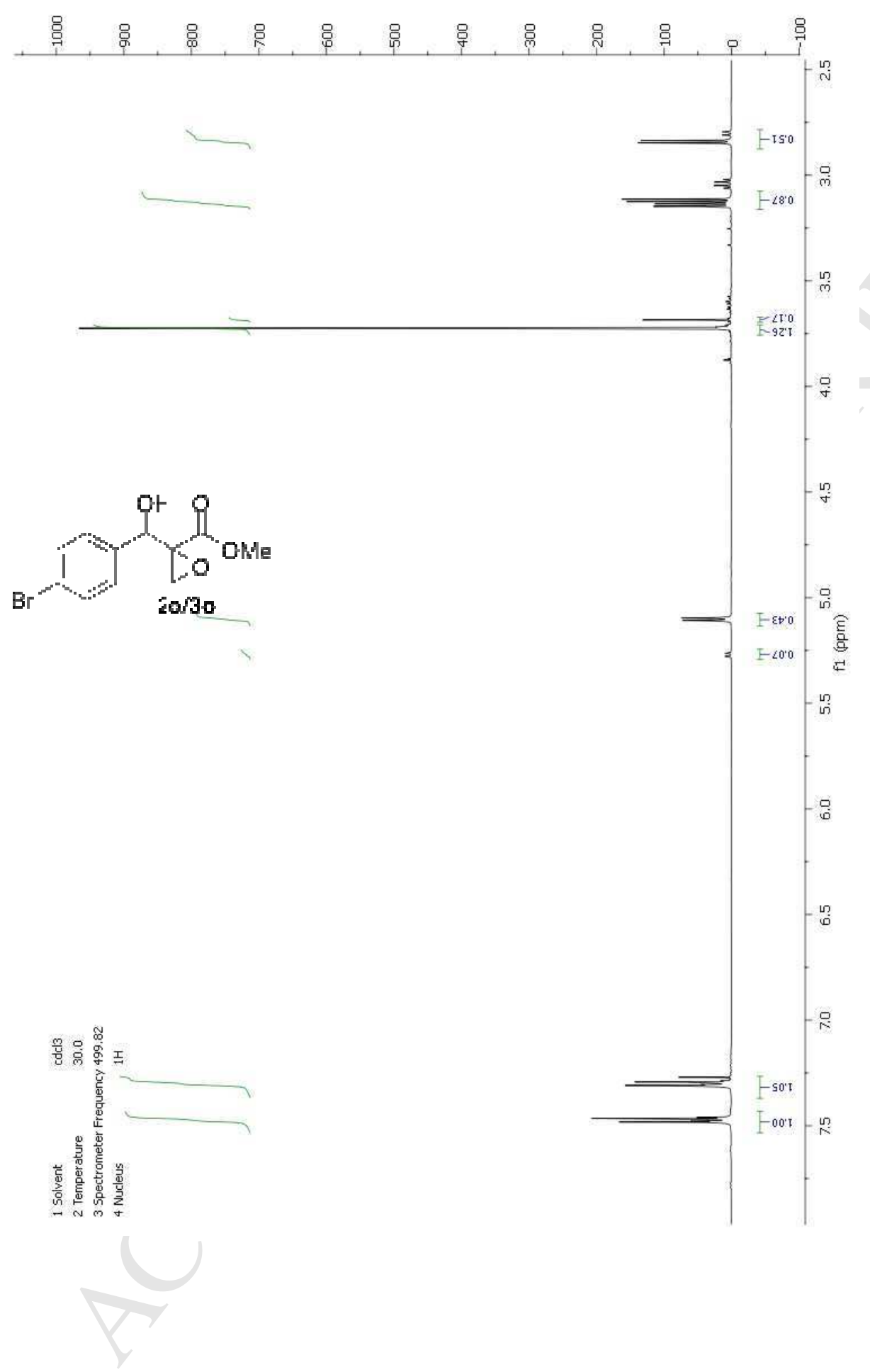




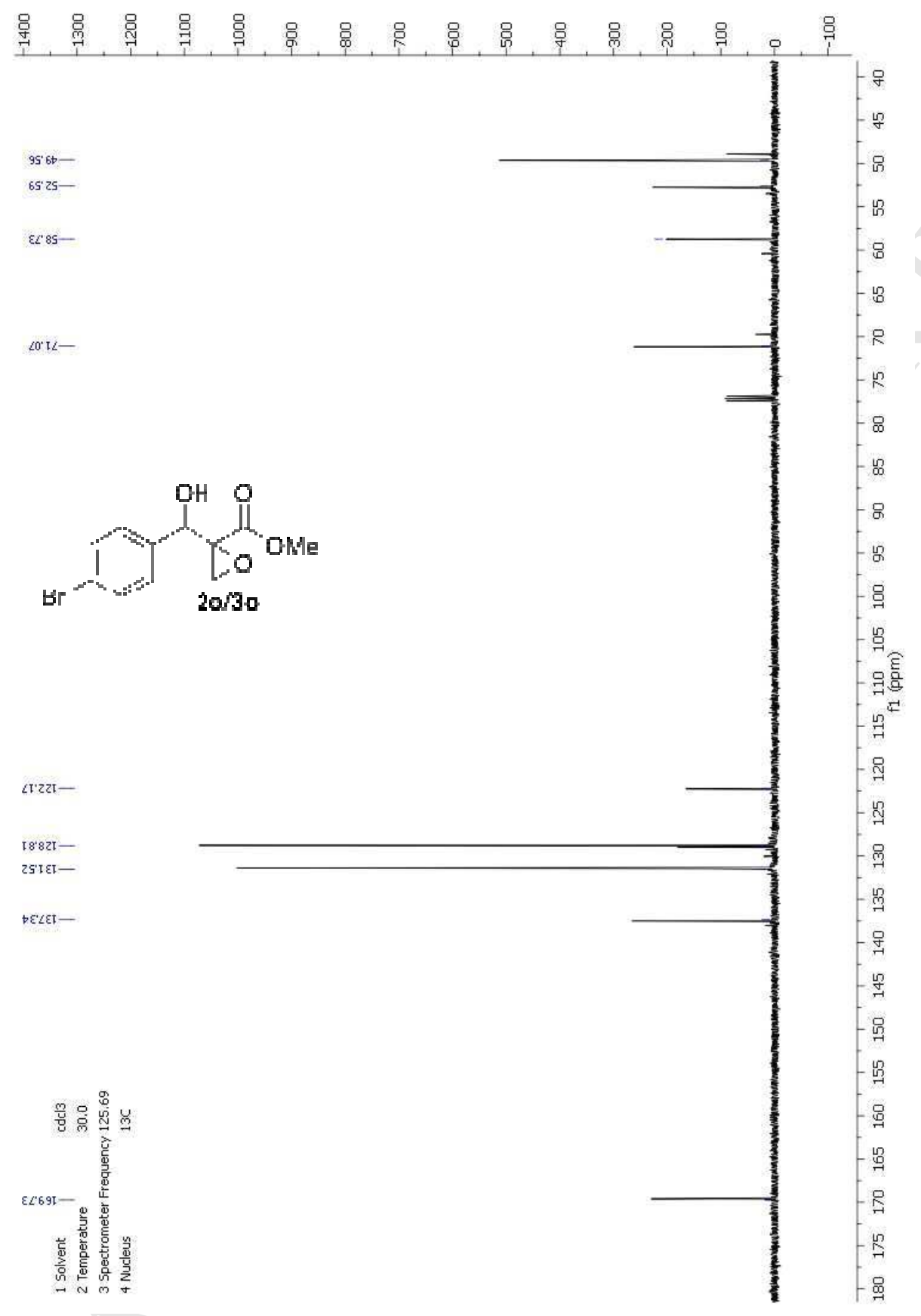




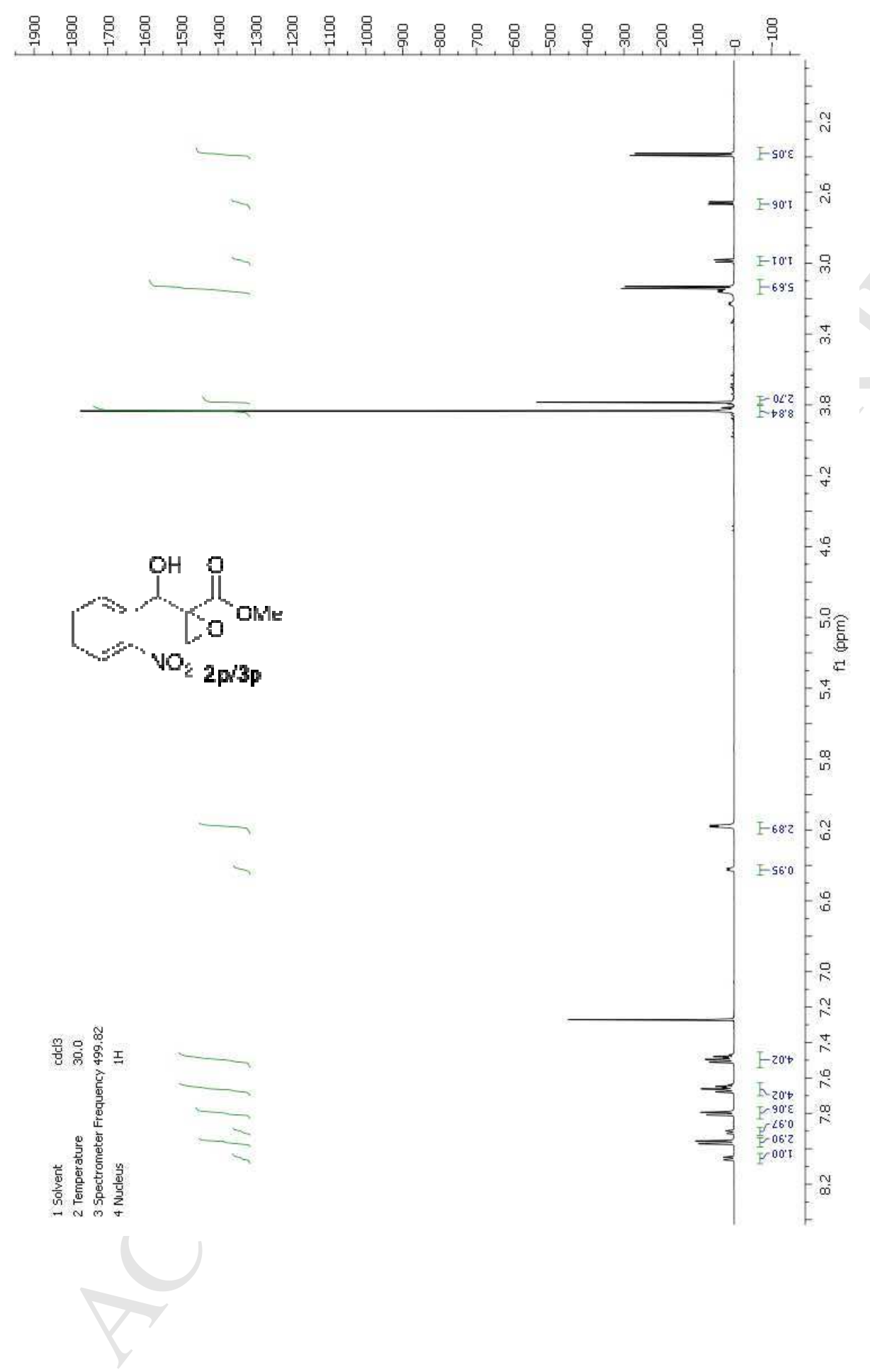




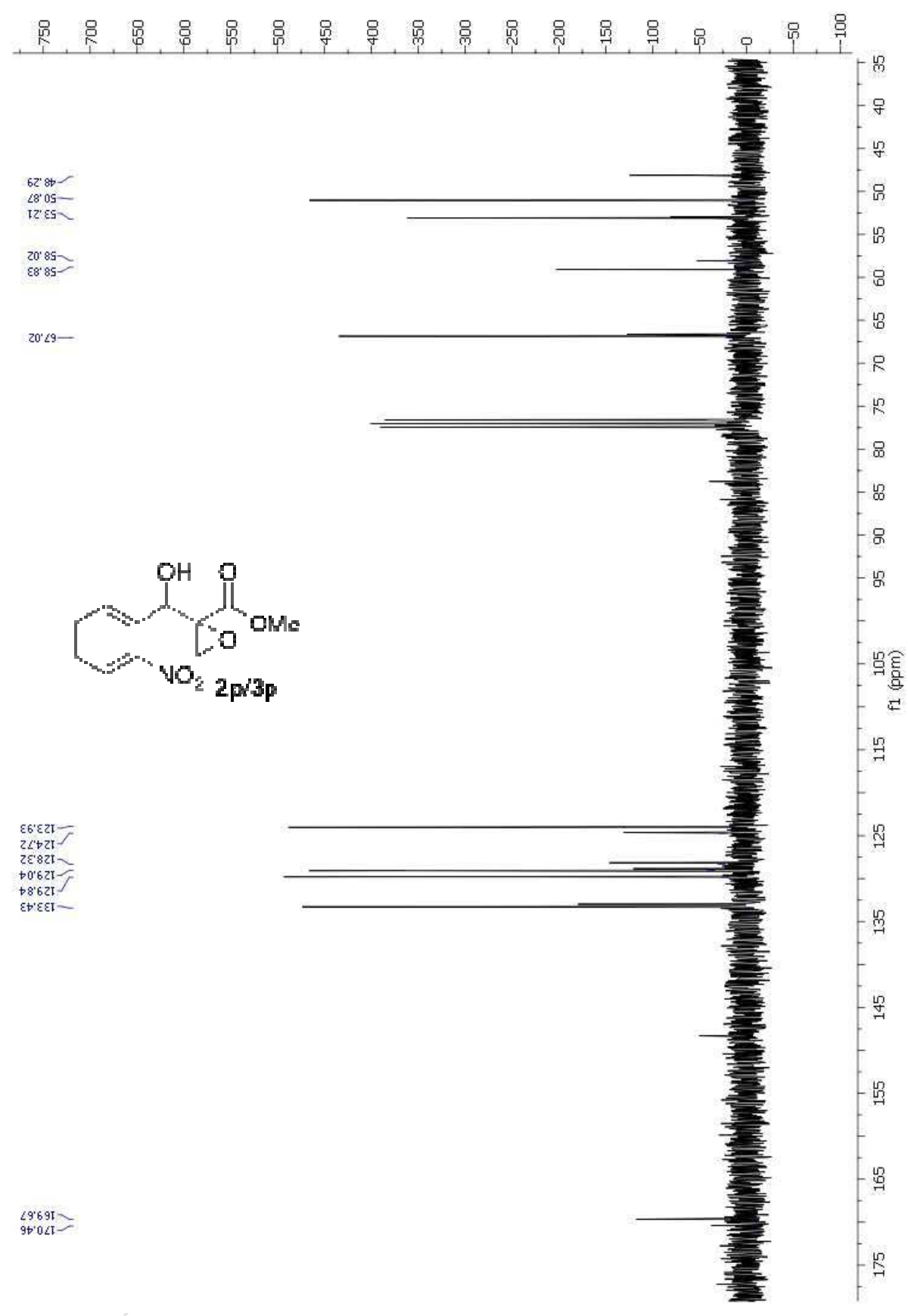




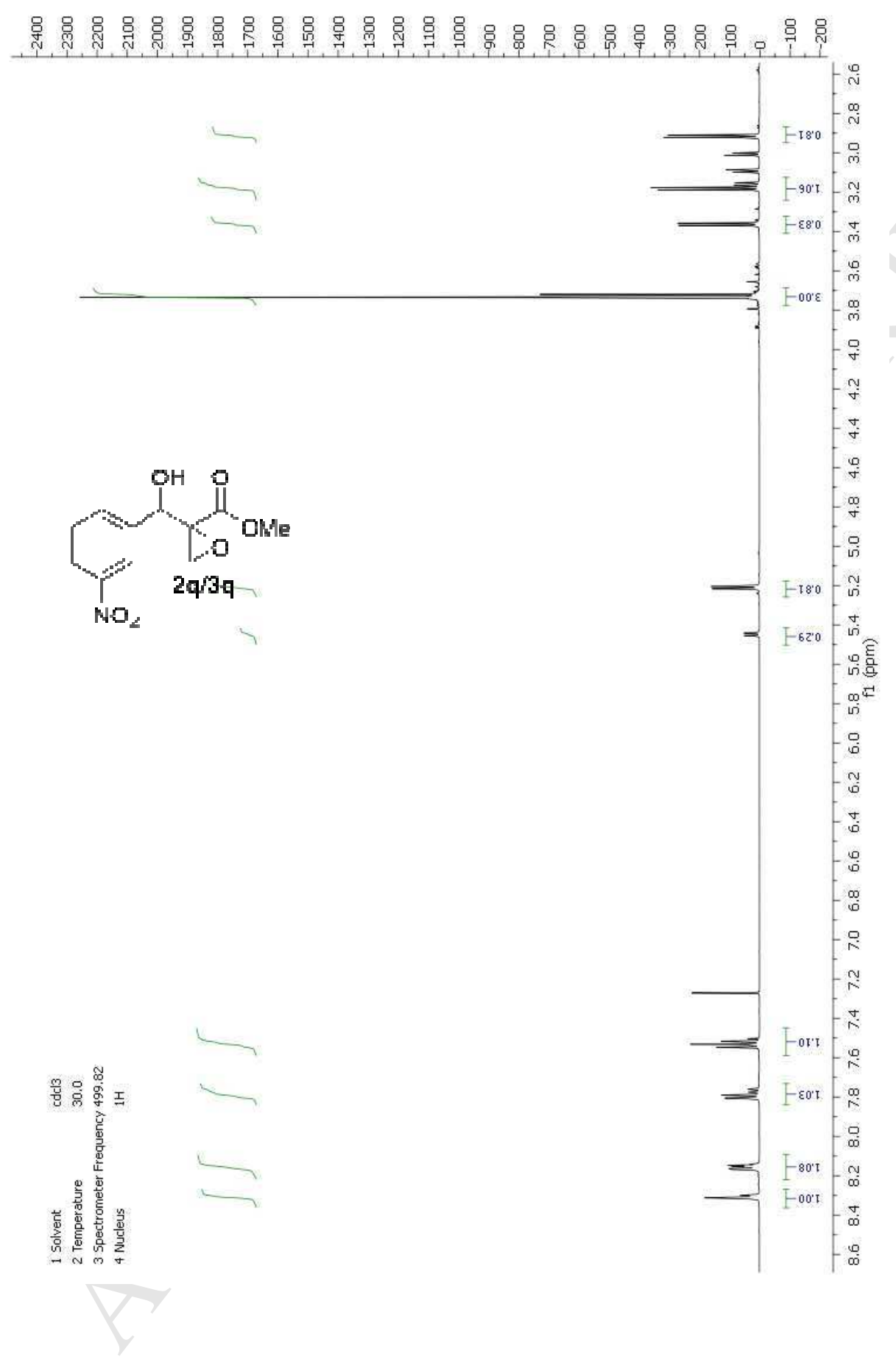




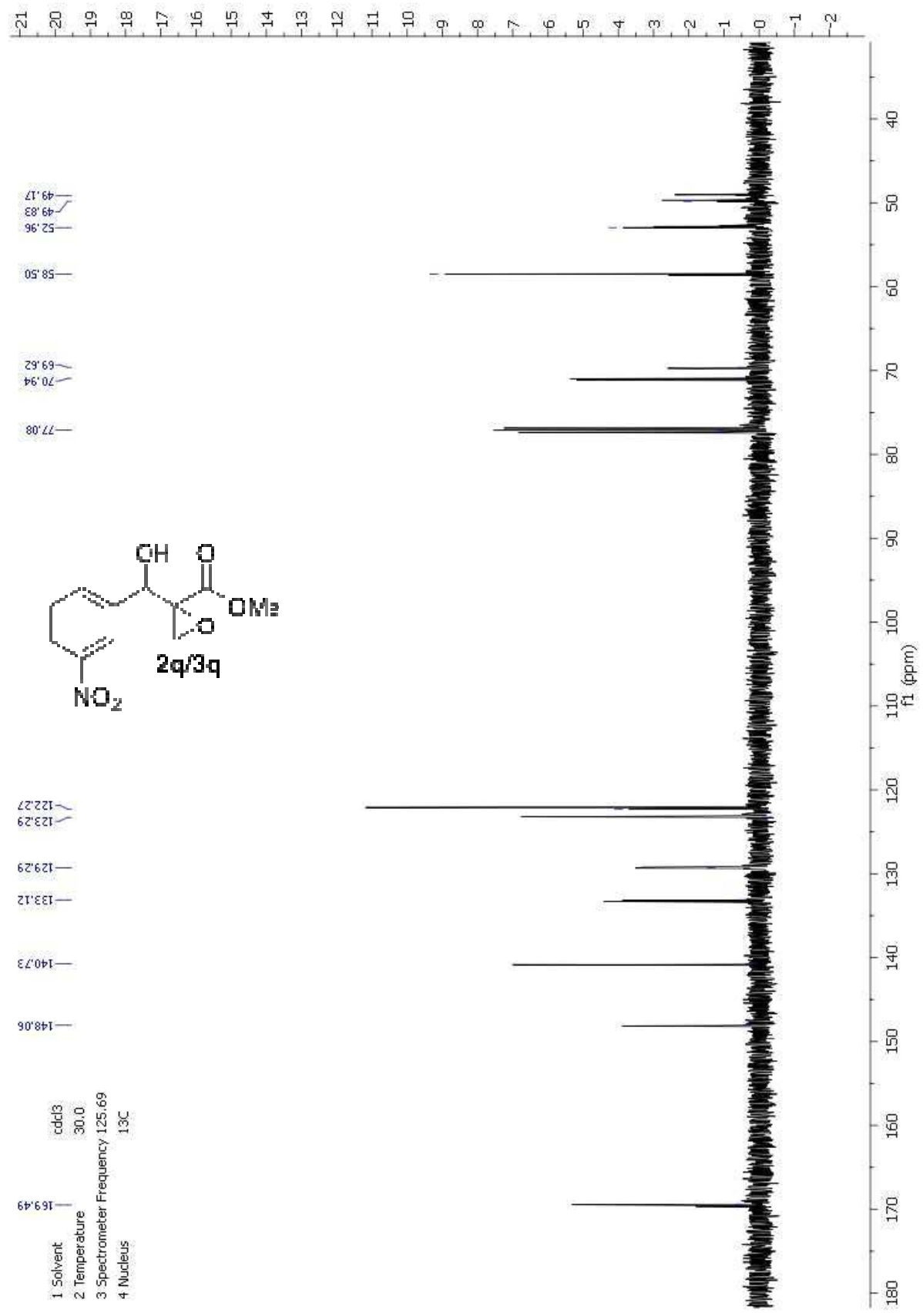




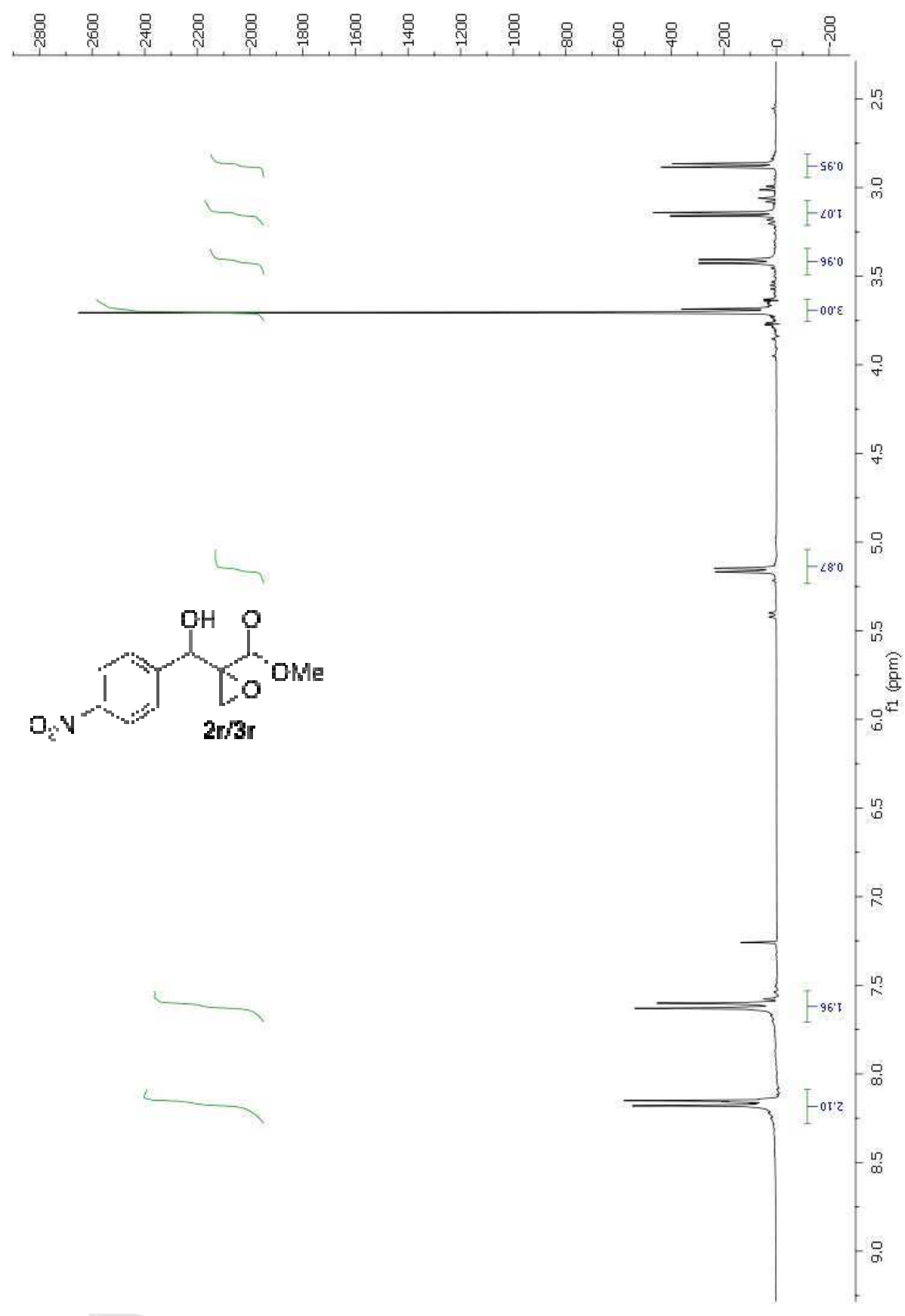




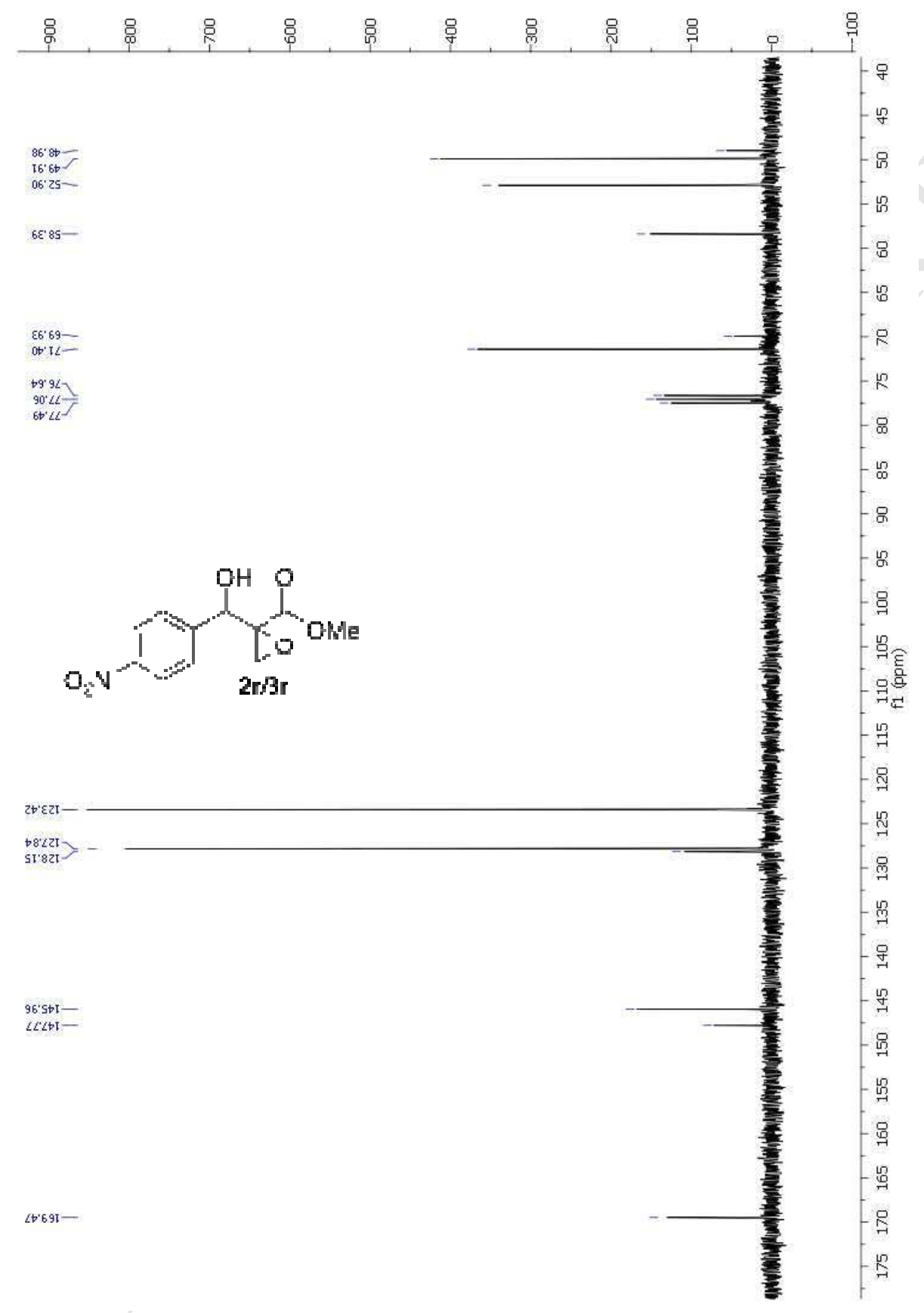




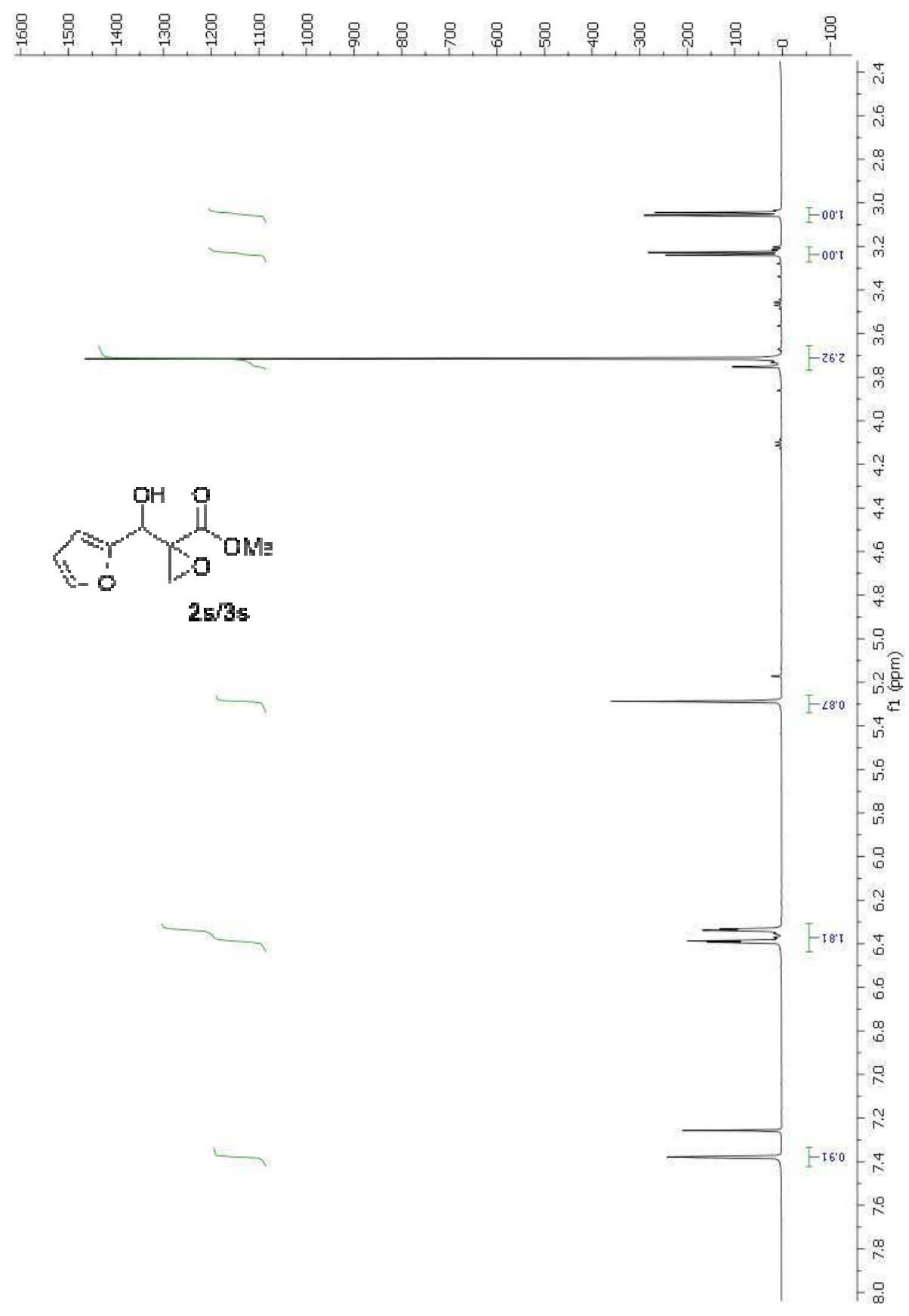




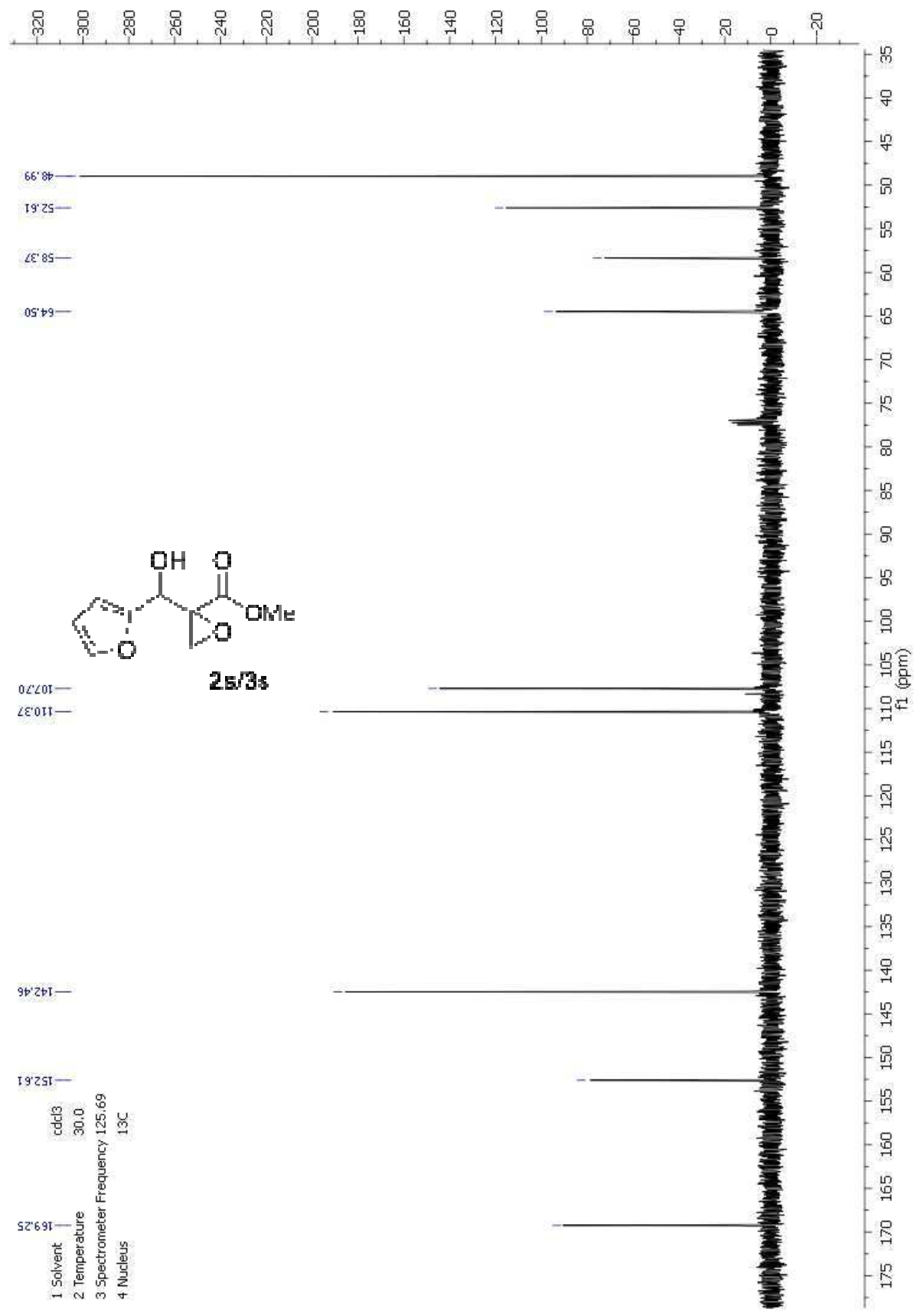




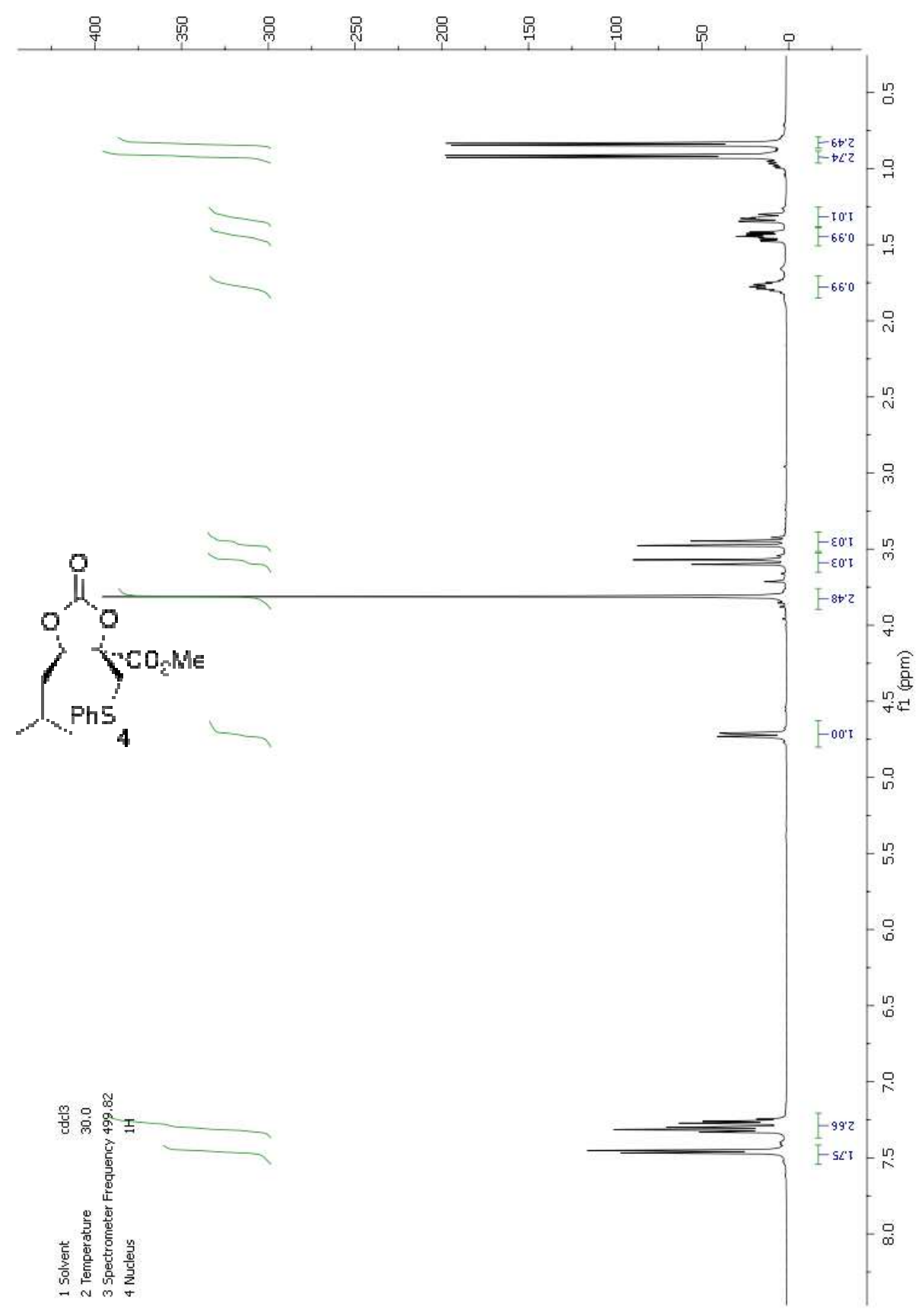




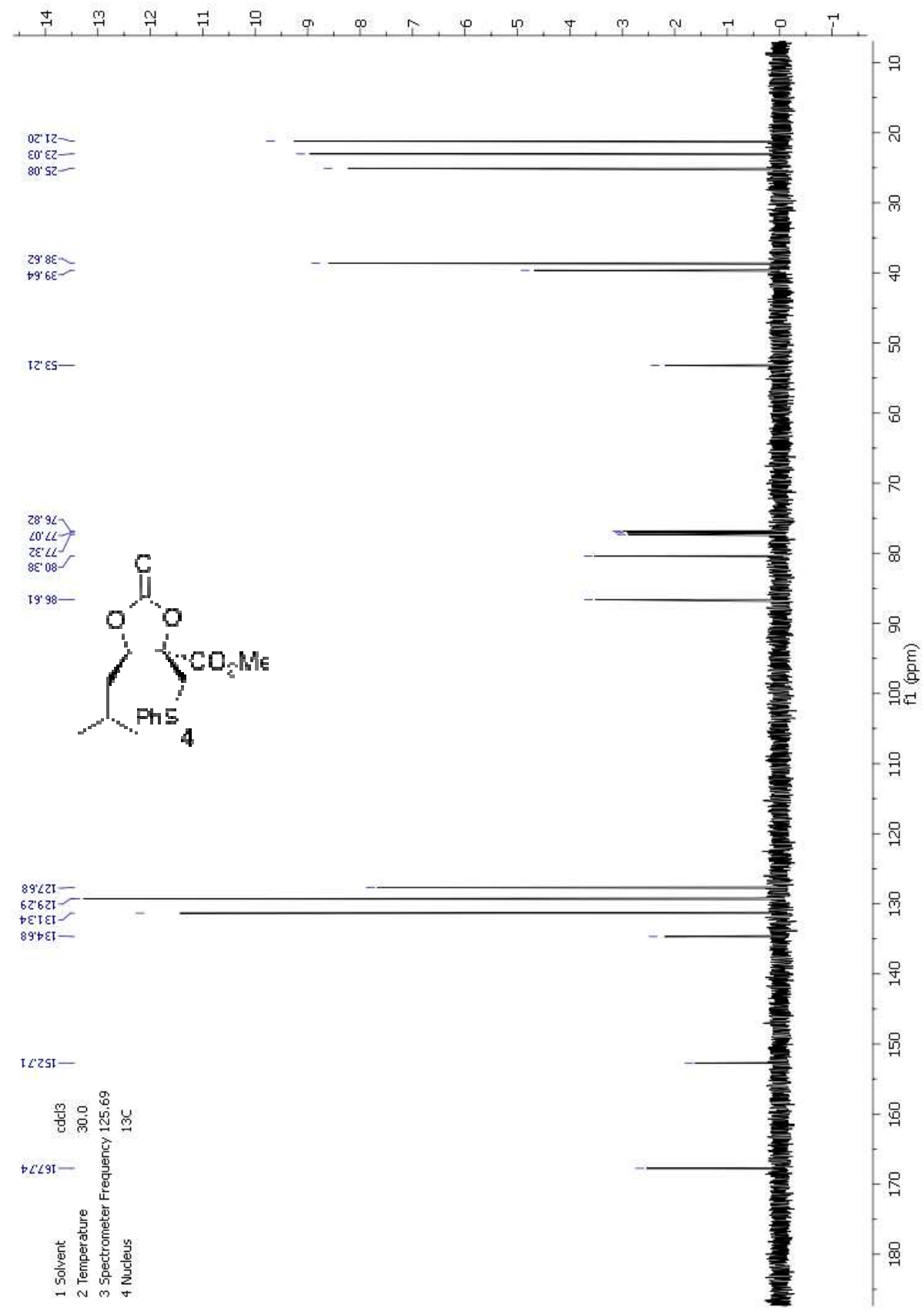




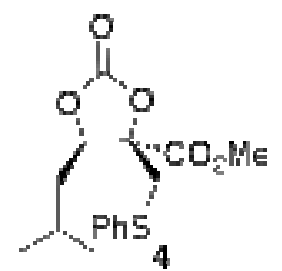




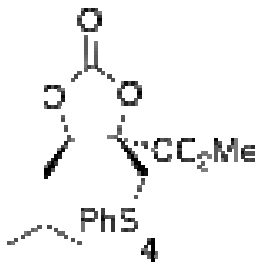




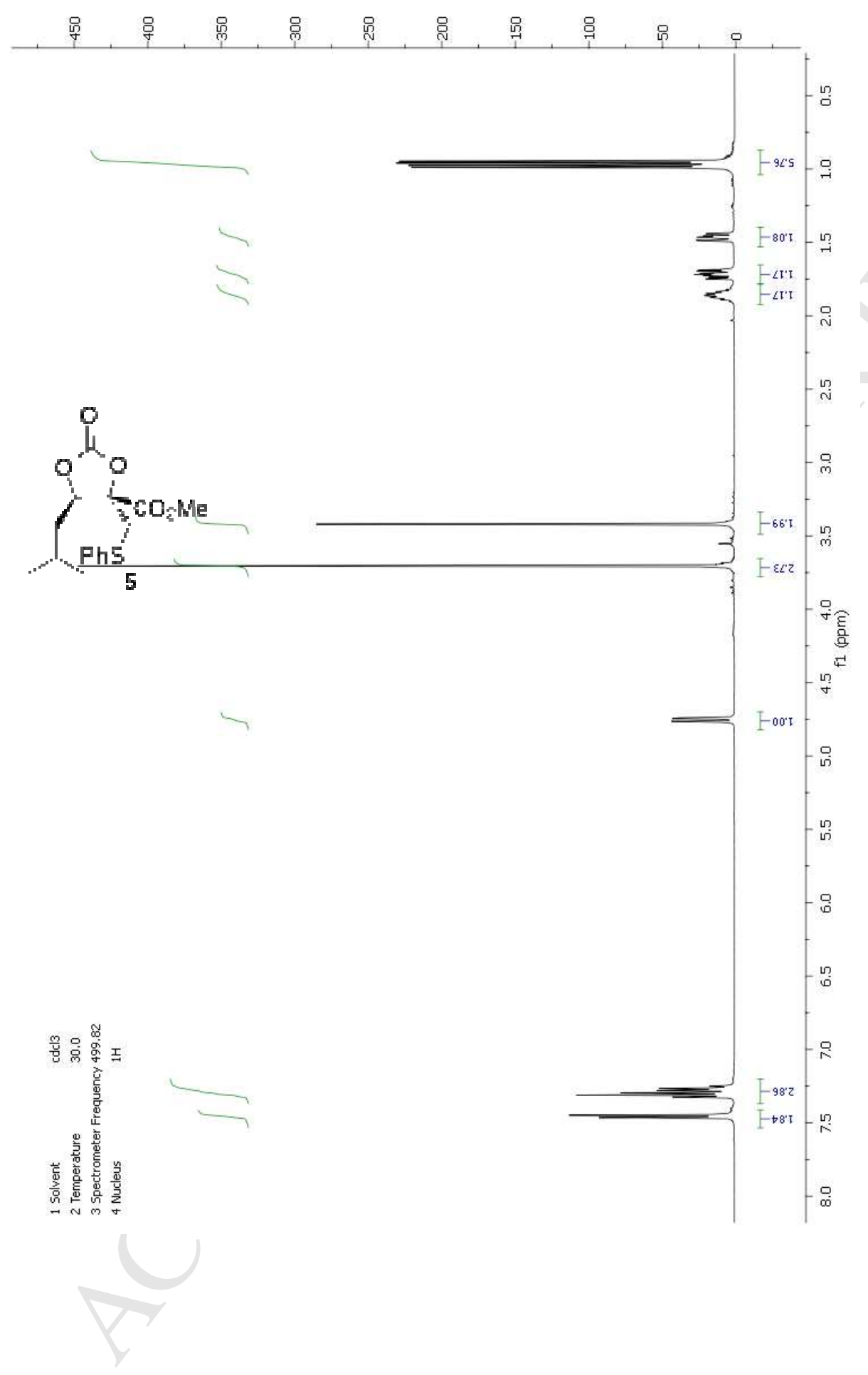




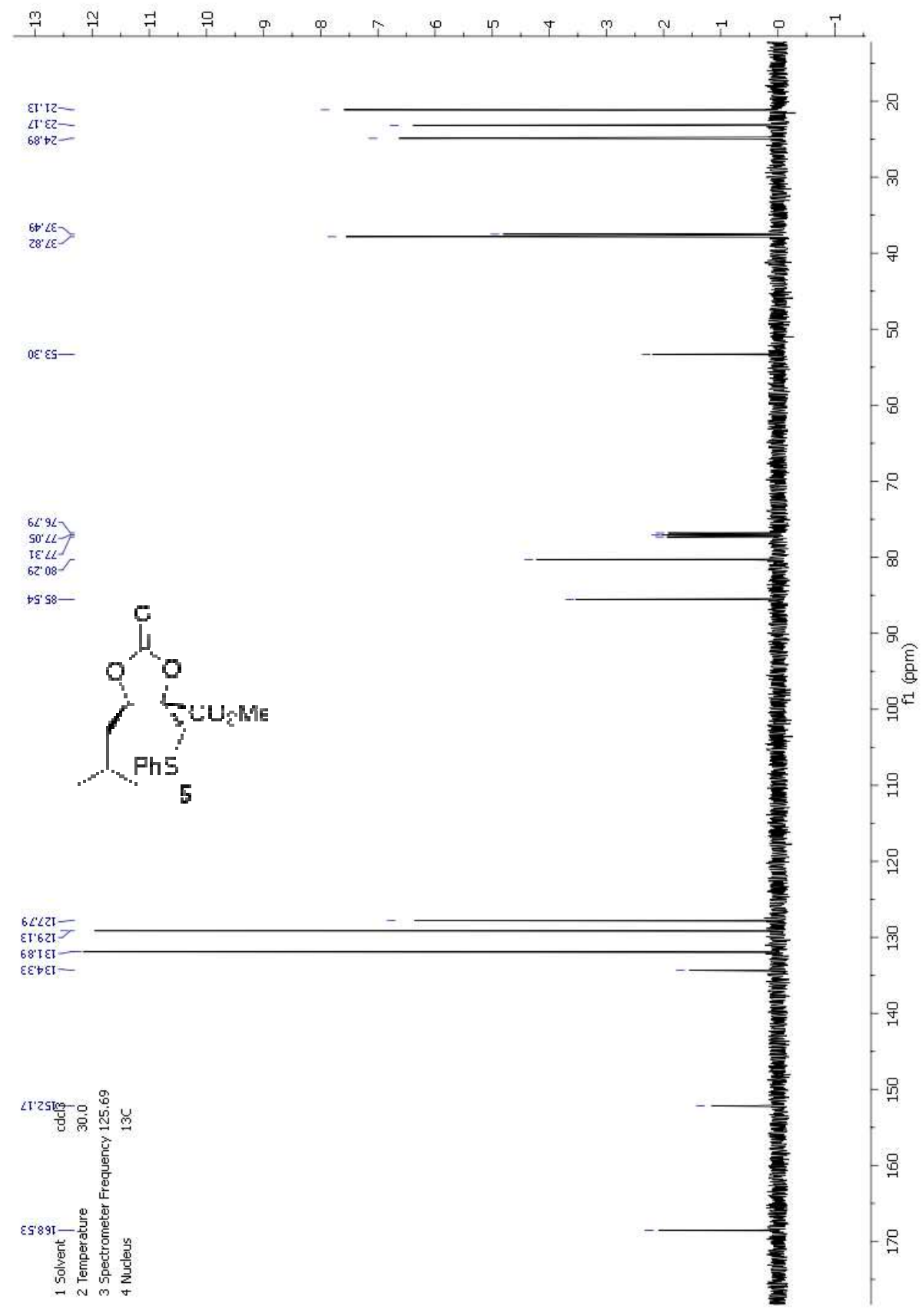




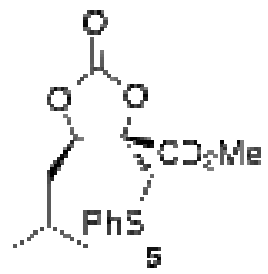




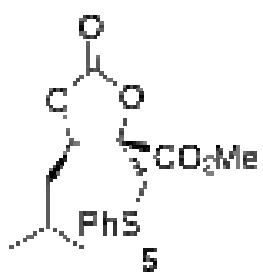




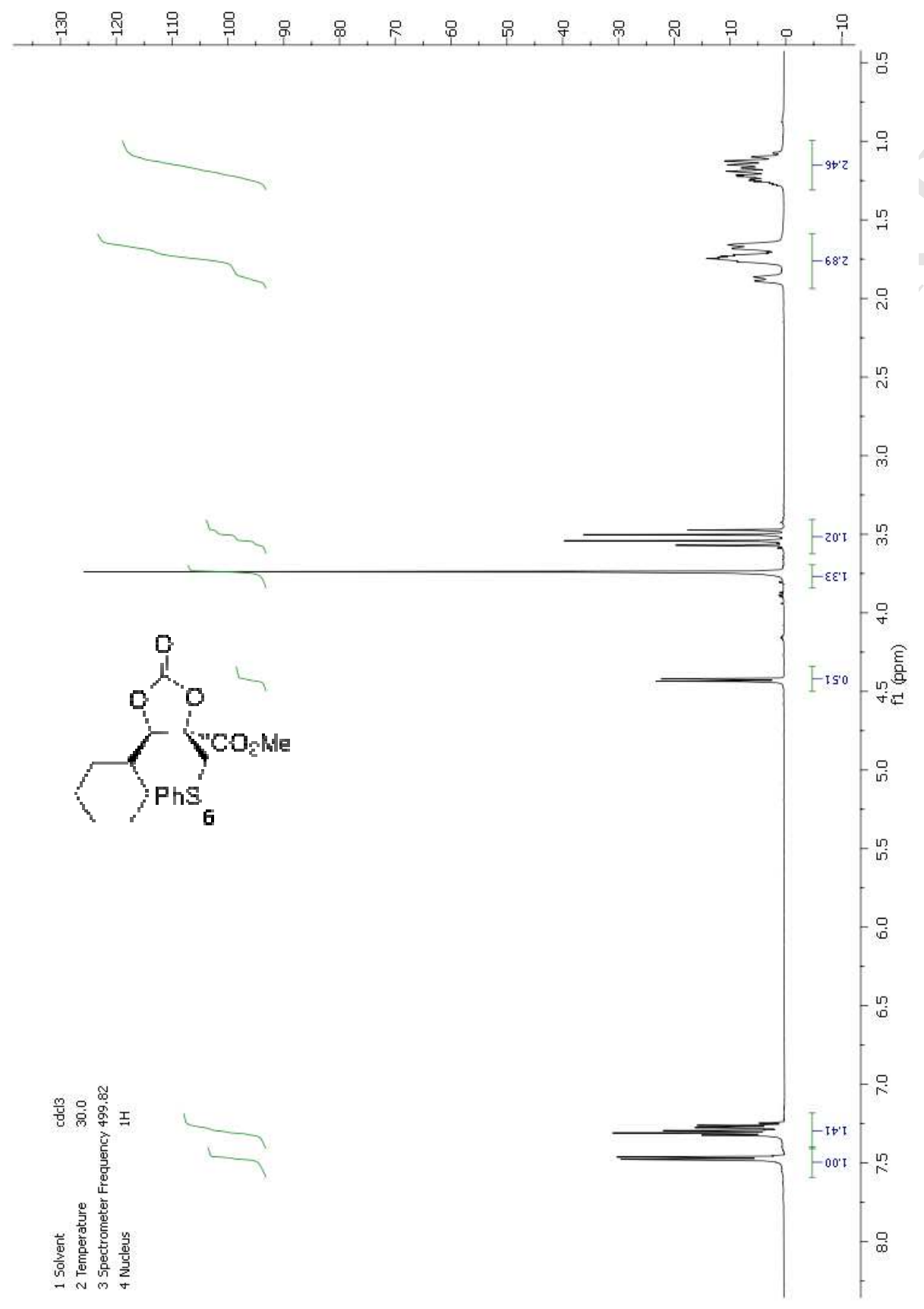




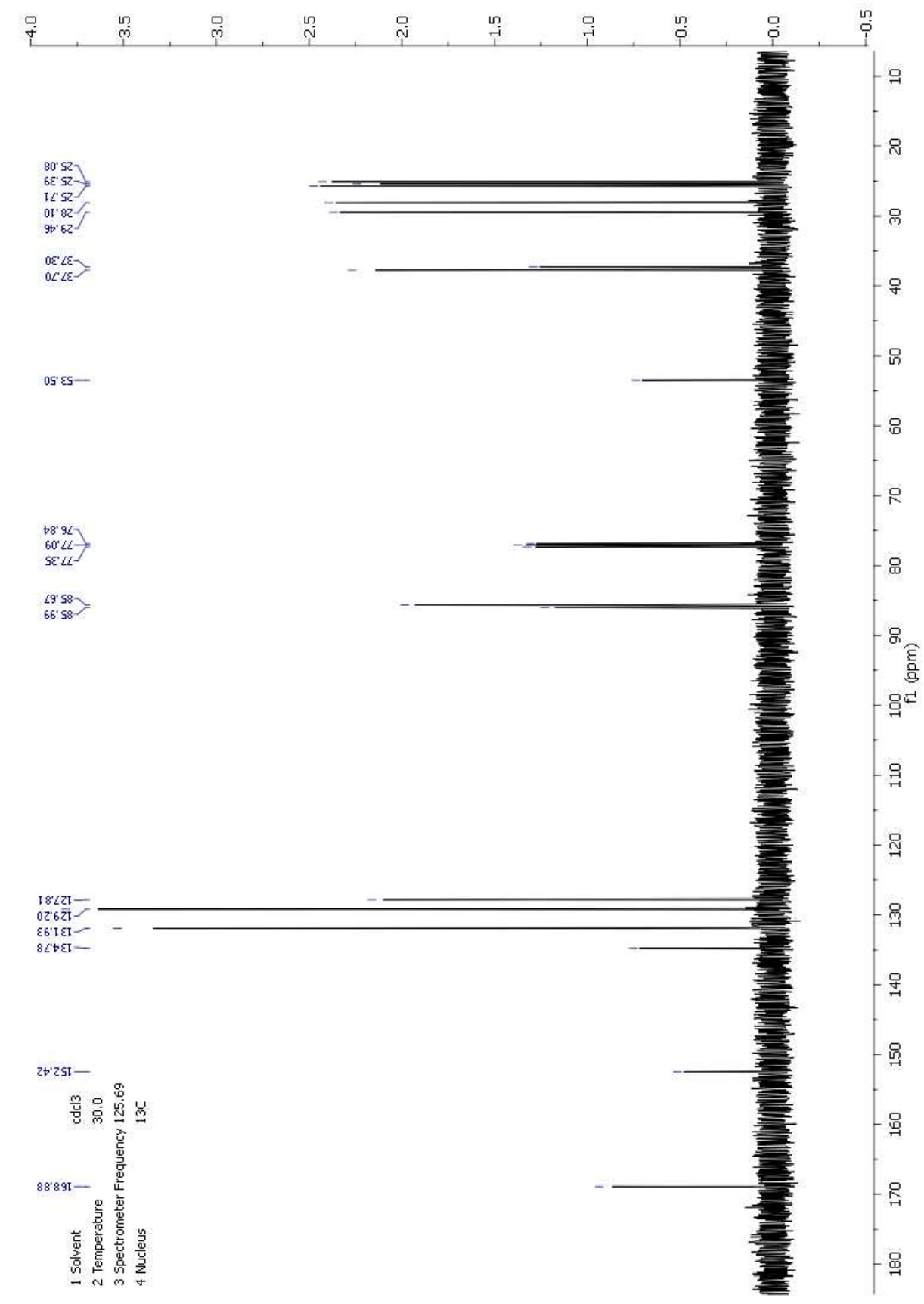




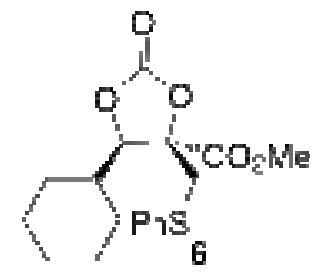




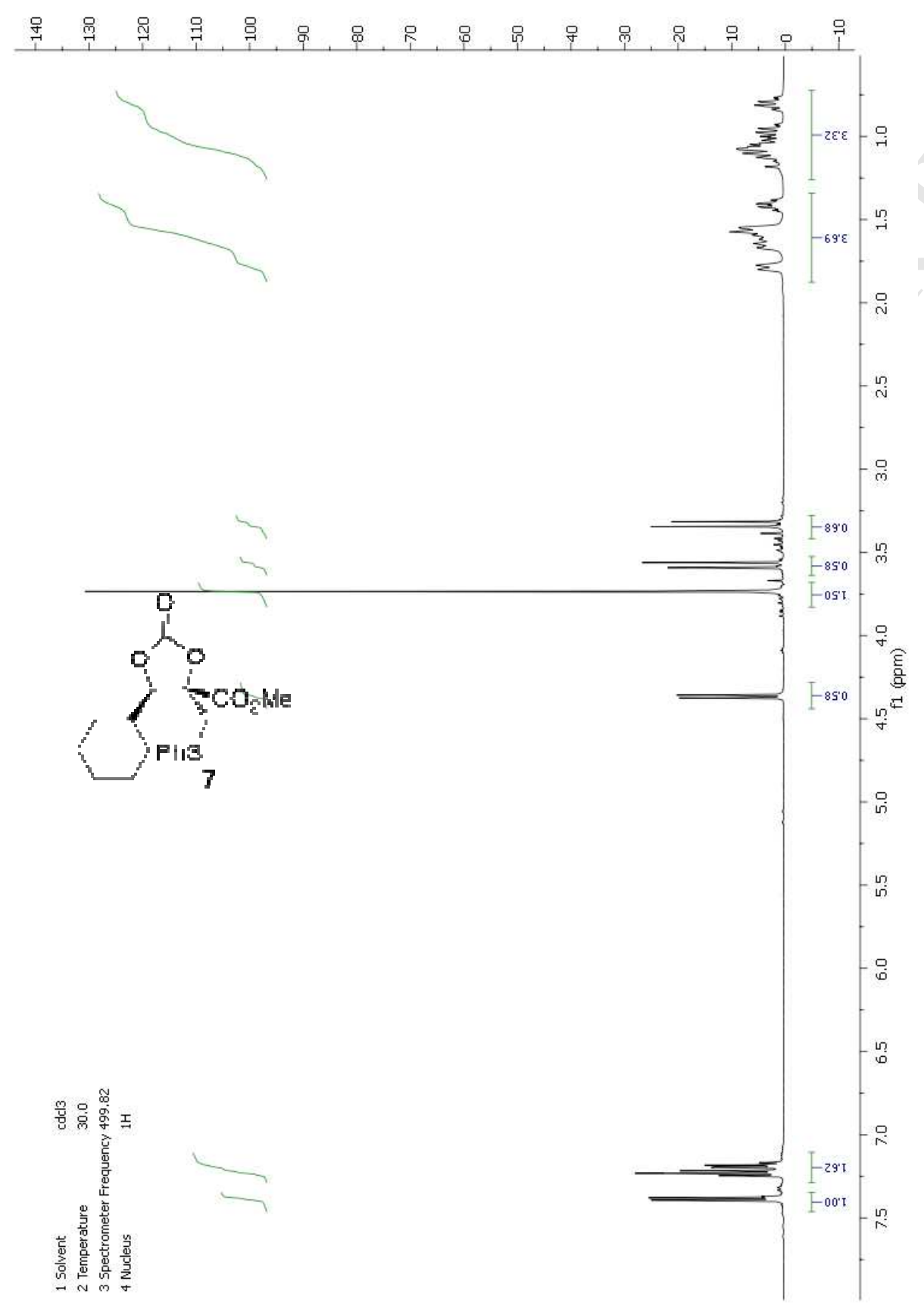




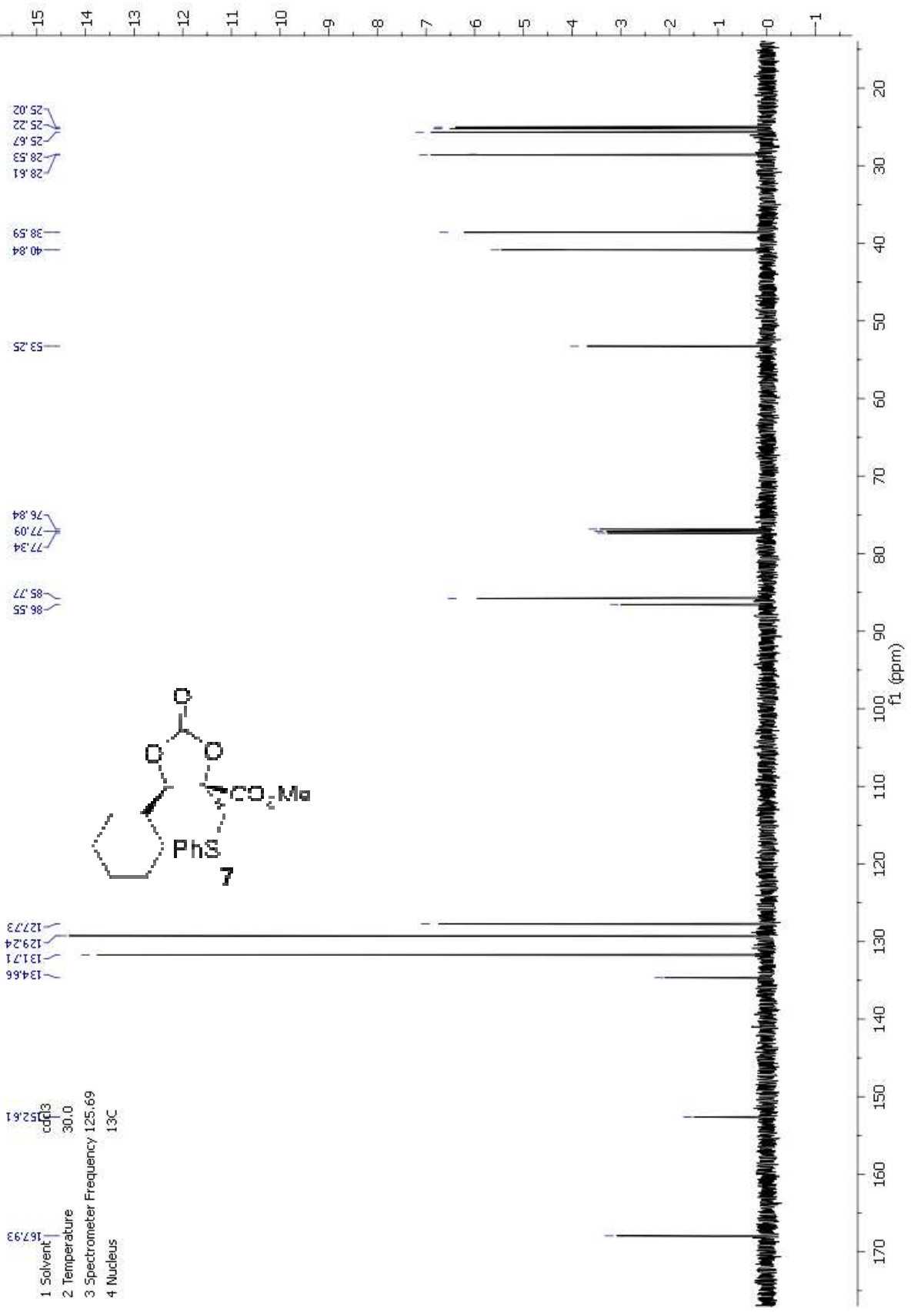




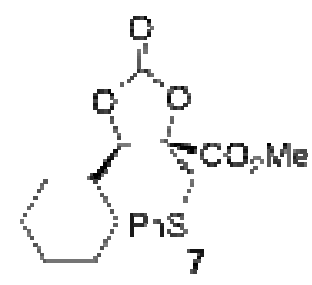

